UCRL-LR-115144

Distribution Category UC-906

\title{
Mean Carrier Transport Properties and Charge Collection Dynamics of Single-Crystal, Natural Type IIa Diamonds From Ion-Induced Conductivity Measurements
}

\author{
S. S. Han \\ (Ph.D. Dissertation)
}

Manuscript date: September 1993

LAWRENCE LIVERMORE NATIONAL LABORATORY University of California - Livermore, California • 94551
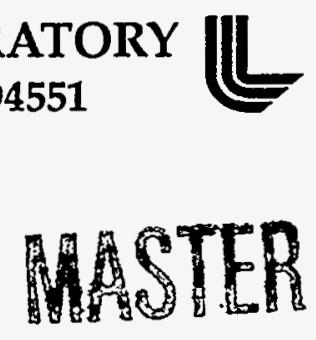

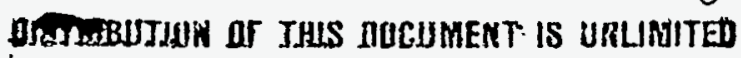




\section{DISCLAIMER}

This report was prepared as an account of work sponsored by an agency of the United States Government. Neither the United States Government nor any agency thereof, nor any of their employees, make any warranty, express or implied, or assumes any legal liability or responsibility for the accuracy, completeness, or usefulness of any information, apparatus, product, or process disclosed, or represents that its use would not infringe privately owned rights. Reference herein to any specific commercial product, process, or service by trade name, trademark, manufacturer, or otherwise does not necessarily constitute or imply its endorsement, recommendation, or favoring by the United States Government or any agency thereof. The views and opinions of authors expressed herein do not necessarily state or reflect those of the United States Government or any agency thereof. 


\section{DISCLAIMER}

\section{Portions of this document may be illegible in electronic image products. Images are produced from the best available original document.}


Mean carrier transport properties and charge collection dynamics of single-crystal, natural type IIa diamonds from ion-induced conductivity measurements

$$
\text { by }
$$

\section{Sung Su Han}

B.S. (University of California at Berkeley) 1985

M.S. (University of California at Berkeley) 1989

A dissertation submitted in partial satisfaction of the requirements for the degree of

Doctor of Philosophy

in

Nuclear Engineering

in the

GRADUATE DIVISION

of the

UNIVERSITY of CALIFORNIA at BERKELEY

Committee in charge:

Professor Stanley G. Prussin, Chair

Doctor Don R. Kania

Professor Eicke R. Weber

Professor Selig N. Kaplan 
The dissertation of Sung Su Han is approved:
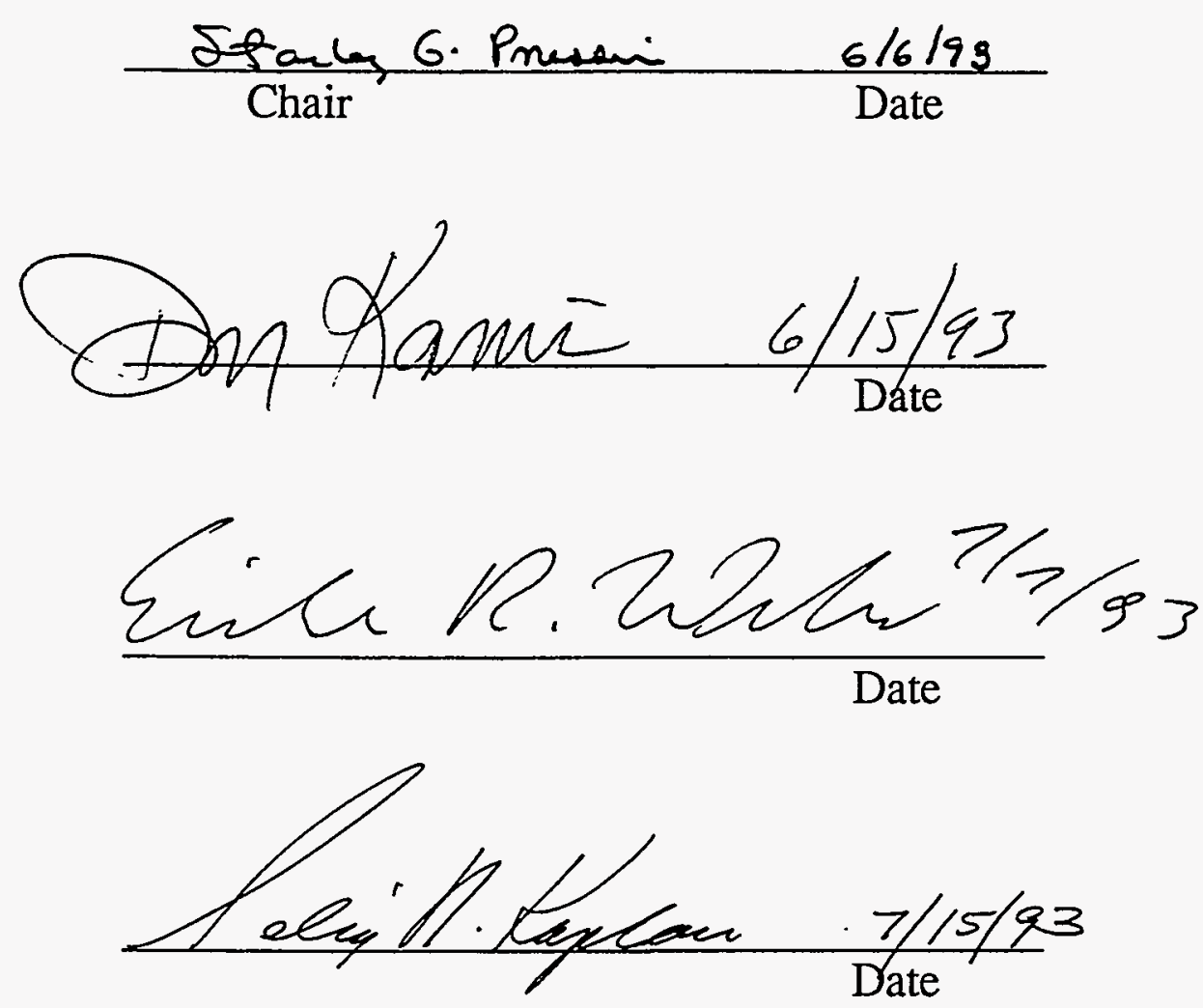

University of California at Berkeley

1993 


\begin{abstract}
Mean carrier transport properties and charge collection dynamics of single-crystal, natural type IIa diamonds from ion-induced conductivity measurements by

Sung Su Han

Doctor of Philosophy in Nuclear Engineering

University of California at Berkeley

Professor Stanley G. Prussin, Chair
\end{abstract}

Ion-induced conductivity has been used to investigate the detector characteristics of diamond detectors. Both integrated-charge, and time-resolved current measurements were performed to examine the mean carrier transport properties of diamond and the dynamics of charge collection under highly-localized and high-density excitation conditions. The integrated-charge measurements were conducted with a standard pulsecounting system with ${ }^{241} \mathrm{Am}$ radioactivity as the excitation source for the detectors. The time-resolved current measurements were performed using a $70 \mathrm{GHz}$ random sampling oscilloscope with the detectors incorporated into high-speed microstrip transmission lines and the excitation source for these measurements was an ion beam of either $5-\mathrm{MeV} \mathrm{He}^{+}$ or $10-\mathrm{MeV} \mathrm{Si}^{3+}$. The detectors used in both experiments can be described as metalsemiconductor-metal (MSM) devices where a volume of the detector material is sandwiched between two metal plates.

A charge collection model was developed to interpret the integrated-charge measurements which enabled estimation of the energy required to produce an electronhole pair $\left(\varepsilon_{\mathrm{di}}\right)$ and the mean carrier transport properties in diamond, such as carrier mobility and lifetime, and the behavior of the electrical contacts to diamond. $\varepsilon_{\mathrm{di}}$ was estimated to be $14.1 \pm 0.5 \mathrm{eV}$, electron and hole mobilities varied in the ranges of about $226-825 \mathrm{~cm}^{2} / \mathrm{V}$-sec and $168-616 \mathrm{~cm}^{2} / \mathrm{V}-\mathrm{sec}$, respectively, and the lifetimes varied in the 
ranges of about 325 - 964 ps for holes and about $38-248$ ps for electrons. In all of the detectors examined, the electron lifetime was found to be shorter in comparison to the hole lifetime, suggesting that preferential electron capture may occur in natural type IIa diamond. Furthermore, the hole contribution to the total collected charge was generally found to be greater by about a factor of 2 then the electron contribution primarily due to its longer lifetime. Furthermore, the electrical contacts appear to behave in a manner similar to non-injecting contacts that have large surface recombination velocities although the $\mathrm{I}-\mathrm{V}$ characteristics of the detectors were linear and indicative of ohmic contacts.

The best fractional resolution resulting from any of the diamond detectors at the highest field $(\approx 220 \mathrm{KV} / \mathrm{cm})$ of operation was about 0.17 . Such poor detector resolution appears primarily due to severe incomplete charge collection. Incomplete charge collection was also apparent as low-energy tails in pulse-height spectra of nearly monoenergetic $\alpha$-particles.

The rise times of the current transients in the time-resolved current experiments were measured to be greater by about an order of magnitude as compared to the $18.6 \pm 0.6$ ps recording system rise time. Therefore, the rise time has been interpreted as being a direct measure of the plasma time. Incomplete charge collection was also found to be severe in the time-resolved current experiments. At the highest electric field of operation $(\approx 100 \mathrm{KV} / \mathrm{cm}$ ), the largest charge collection efficiencies were measured to be about 0.1 and 0.2 for $\mathrm{Si}^{3+}$ - and $\mathrm{He}^{+}$-ion excitations, respectively. Also, the charge that was collected was about a factor of 2.5 greater when the ion tracks were parallel to applied electric field then when the tracks were normal to the applied field.

We have developed a temporally- and spatially-dependent one-dimensional carrier transport model to interpret the time-resolved current measurements and to examine the dynamics of charge collection under the intense localized excitations that are represented by heavy ion-excitations. A one-dimensional model may be adequate in describing the response of a diamond RIC detector to ions such as helium-ions at least qualitatively 
where the excitation density may be low enough. But for heavier ions, such as siliconions, where the excitation density may be much greater than helium-ions, the model appears to be inadequate and may require multi-dimensional transient model. The large initial current predicted by the model is not seen experimentally.

Stadg 6.Presen 7/2/83

Professor Stanley G. Prussin, Chair

Date 



\section{Table of Contents}

List of Figures

List of Tables

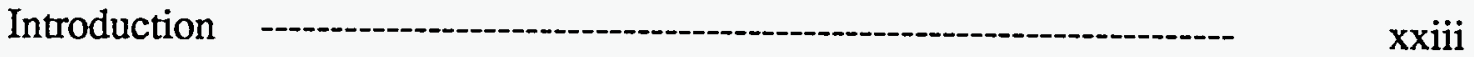

Acknowledgments -

Chapter 1: Theory of radiation-induced conductivity

1.1 Brief history and background on the use of diamonds for radiation detection $\quad$ - 1

1.2 Radiation interaction with matter

1.3 Radiation-induced conductivity (RIC) -

1.4 Principles of detector operation

1.4.1 Dielectric relaxation

1.4.2 Relaxation through recombination

1.5 Electrical contacts to diamond

1.5.1 Ohmic contacts 19

1.5.2 Schottky contacts $\quad$ -

Chapter 2: Detector processing and experimental arrangements

2.1 Detector preparation $\quad 26$

2.2 Detector processing and the experimental arrangement for integrated-charge measurements $\quad 27$

2.3 Detector fabrication and experimental arrangement for the time-resolved current experiments $\quad 39$

2.3.1 Detector fabrication

2.3.2 Experimental arrangement $\quad 52$

Chapter 3: Integrated-charge experiments - -

3.1 Experimental results $\quad 59$ 
3.1.1 Experimental results analysis

3.1.2 Total mean collected charge results

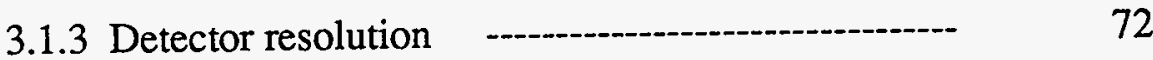

3.2 Charge collection - 76

3.2.1 Model for mean collected charge

3.2.2 Comparison between model and experiment for the collected charge -

3.3 Detector resolution

3.3.1 Resolution effects of incomplete charge collection $\quad 107$

3.3.2 Comparison between calculated and experimental detector resolutions

Chapter 4: Time-resolved current experiments

4.1 Experimental results

4.1.1 Experimental conditions

4.1.2 Results from the interaction of $5-\mathrm{MeV} \mathrm{He}^{+}$ions with the detector HE6. 4

4.1.3 Results from the interaction of $10-\mathrm{MeV} \mathrm{SI}^{3+}$ ions with the detector SI6.9

4.2 Modeling of time-resolved current transients

4.2.1 Model assumptions

4.2.2 Model equations

4.2.3 Numerical solution to model equations

4.3 Model calculations and comparisons with experimental results

4.3.1 5-MeV He+-ion excitation

4.3.2 $10-\mathrm{MeV} \mathrm{Si}^{3+-}$-ion excitation

Chapter 5: Conclusions 
Chapter 6: Is there life after this thesis? (Future research directions)

Appendix A: Photolithography and lift-off and wet/dry etch

processes: the recipes

Appendix B: Experimental arrangement used for intrinsically excited transient photoconductivity measurements

Appendix C: $\mathrm{O} \underline{\text { th }}$ order calculation of the induced charge in a radiation-induced conductor under 1) $\infty$-carrier recombination at the boundaries and 2) carrier injection at the boundaries

Appendix D: $5 \mathrm{MeV}$ alpha-particle damage of single crystal, natural type IIa and polycrystalline CVD diamonds

Appendix E: Calculation of the ratio of the peak carrier density to the mean for carriers that are distributed in a Gaussian distribution radially within an ion track 


\section{List of figures}

Figure 1.3.1: Schematic of a general radiation detection system.

Figure 1.4.1: Energy valley representation of the direct band-to-band recombination.

Figure 1.4.2: Energy valley representation of the indirect band-to-band recombination.

Figure 1.4.3: Four possible defect level assisted processes.

Figure 1.5.1: A) Contact geometry used to measure the Schottky diode behavior of 50 $\mu \mathrm{m}$ thick polycrystalline CVD diamond with $\mathrm{Au}$ as one contact and $\mathrm{Ti} / \mathrm{Pt}$ as the other contact. B) I-V characteristics of the diode shown in A).

Figure 2.2.1: Shadow mask arrangement the electrical contact metalization on diamond detectors

Figure 2.2.1: Shadow mask arrangement the electrical contact metalization on diamond detectors

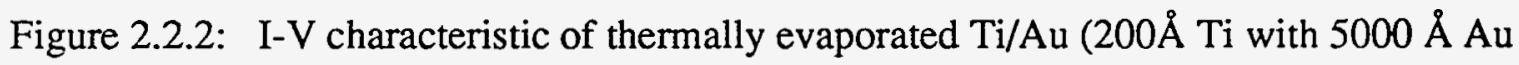
overlayer) contacts on SYM14m.

Figure 2.2.3: $\quad \mathrm{I}-\mathrm{V}$ characteristic of sputtered Ti/Pt (200 $⿱$ Ti with $5000 \AA$ Pt overlayer) contacts on SYM25m.

Figure 2.2.4: Schematic of the sample holder used in integrated-charge experiments

Figure 2.2.5: Schematic of the integrated-charge experimental arrangement

Figure 2.2.6: The effect of $5000 \AA$ thick gold foil on $5.48-\mathrm{MeV} \alpha$-particles from an ${ }^{241}$ Am source.

Figure 2.3.1: Schematic and photograph of complete detector packages used for the time-resolved current experiments.

Figure 2.3.2: Schematics of two different transmission lines.

Figure 2.3.3: Gold-conductor loss $\left(\alpha_{c}\right)$ and skin depth $(\delta)$ as a function of propagating signal frequency with alumina as the dielectric substrate and with the ratio $w / h=1$. 
Figure 2.3.4: Comparison of the waveforms obtained after differentiating a step-pulse of 20-ps rise time, and after transmitting the step pulse through the microstrip.

Figure. 2.3.5: The transmitted pulse with the same input pulse used to obtain Fig. 2.3.4

Figure 2.3.6 : Example of transient photoconductive signal taken with a 6-ps UV laser excitation of one of the detectors used in the time-resolved experiments.

Figure 2.3.7: Schematic for the time-resolved experimental arrangement.

Figure 2.3.8: Photographs of the vacuum chamber and its attachments.

Figure 2.3.9: The output pulse after propagating a $20 \mathrm{ps}$ Gaussian pulse through all of the cables and connectors excluding the detector package.

Figure 2.3.10: The output pulse after propagating a 20-ps step pulse through all of the cables and connectors including through the gap on the detector.

Figure 3.1.1: Pulse-height spectra for detector SYM14m as a function of bias voltage, $\mathrm{V}_{\mathrm{b}}$.

Figure 3.1.2: Pulse-height spectra for detector SYM25m as a function of bias voltage, $\mathrm{V}_{\mathbf{b}}$.

Figure 3.1.3: Pulse-height spectra for detector SYM70 $\mathrm{m}$ as a function of bias voltage, $\mathrm{V}_{\mathrm{b}}$.

Figure 3.1.4: Pulse-height spectrum from detector SYM25m at an applied electric field of $\mathrm{E}=-4 \mathrm{KV} / \mathrm{cm}$.

Figure 3.1.5: Pulse-height spectrum from SYM25m at an applied electric field of $E \simeq-100 \mathrm{KV} / \mathrm{cm}$.

Figure 3.1.6: Total mean collected charge as a function of applied electric field for SYM14m. 
Figure 3.1.7: Total mean collected charge as a function of applied electric field for detector SYM25m.

Figure 3.1.8: Mean collected charge as a function of applied bias reported by Kozlov, et al.

Figure 3.1.9: Total mean collected charge as a function of applied electric field for SYM70m.

Figure 3.1.10: Ratio of total mean collected charge for positive polarity to that of negative polarity for diamond detectors varying in thickness.

Figure 3.1.11: Fractional resolution, DR, of SYM14m as a function of applied electric field.

Figure 3.1.12: Fractional resolution, DR, of SYM25m as a function of applied electric field.

Figure 3.1.13: Fractional resolution, DR, of SYM70m as a function of applied electric field.

Figure 3.2.1: Schematic of model for the detectors studied.

Figure 3.2.2: The TRIM-calculated stopping power distribution of 5.48-MeV $\alpha$ particle in diamond.

Figure 3.2.3: Charge collection efficiency, $\eta$, as a function of applied electric field where both contacts are non-injecting.

Figure 3.2.4: Illustrations of charge collection efficiencies for two non-injecting contacts as a function of the ratio of carrier mean free path to detector width (electrode separation) $\lambda / \mathrm{L}$ and the ratio of the mean ion range to detector width.

Figure 3.2.5: Charge collection efficiency, $\eta$, as a function of electrical contacts using constant stopping power.

Figure 3.2.6: Charge collection efficiency, $\eta$, as a function of electrical contacts using TRIM-calculated stopping power. 
Figure 3.2.7: Comparison betweèn calculated charge collection efficiencies with the constant stopping power assumption and the stopping power calculated with TRIM.

Figure 3.2.8: Comparison between the model and the experimental data for SYM25m assuming both contacts to be injecting.

Figure 3.2.9: Comparison between the model and the experimental data for SYM25m assuming one contact to be electron injecting and the other non-injecting to holes.

Figure 3.2.10: Comparison between the model and the experimental data for SYM25m assuming one contact to be hole injecting and the other to non-injecting to electrons

Figure 3.2.11: Comparison between the model and the experimental data for SYM25m using non-injecting contacts for the both contacts.

Figure 3.2.12: Comparison between the experiment and the model for SYM14m.

Figure 3.2.13: Comparison between the experiment and the model for SYM70m.

Figure 3.2.14: Comparison between the model and the experimental data for SYM14m using non-injecting contacts for both contacts.

Figure 3.2.15: Comparison between the model and the experimental data for SYM70m using non-injecting contacts for both contacts.

Figure 3.2.16: Comparison between calculated mean free paths for different combinations of contacts with laser measurements for SYM25m.

Figure 3.2.17: Comparison between calculated mean free path and the laser measurements for SYM25m assuming that both contacts are noninjecting.

Figure 3.2.18: Laser data on mean carrier lifetime as a function of applied electric field for SYM25m. 
Figure 3.2.19: Comparison between experimental and calculated ratios of the mean collected charge for the positive polarity to that for negative polarity as a function of the absolute value of the applied electric field, IE I.

Figure 3.2.20: Ratio of mean energy required to produce an electron-hole pair, $\varepsilon$, to band gap, $E_{G}$, as a function of $E_{G}$.

Figure 3.3.1: Calculated fractional resolution, $D R$, as a function of applied electric field and bias polarity.

Figure 3.3.2: Calculated fractional resolution, DR, as a function of applied electric field and bias polarity with $\mu_{h} \tau_{k}=\mu_{e} \tau_{e}=\mu \tau$.

Figure 3.3.3: Calculated fractional resolution, $D R$, as a function of applied electric field and bias polarity with $\mu_{h} \tau_{h}=\mu_{e} \tau_{e}=\mu \tau$.

Figure 3.3.4: Fractional resolution, DR, as a function of applied electric field, bias polarity and the detector width, $\mathrm{L}$.

Figure 3.3.5: Comparison between fractional resolution calculated with constant and non-constant stopping powers for $\alpha$-particles.

Figure 3.3.6: Relative difference between non-constant and constant stopping powers as a function of electric field.

Figure 3.3.7: Comparison between calculation and experiment for detector SYM14m.

Figure 3.3.8: Comparison between calculation and experiment for detector SYM25m.

Figure 3.3.9: Comparison between calculation and experiment for detector SYM70m.

Figure 4.1.1: Average time-resolved current pulses from detector system HE6.4 excited with 5-MeV He ions.

Figure 4.1.2: Stopping power and ion-induced vacancy concentration distributions for 5-MeV He+-ion as calculated with the code TRIM.

Figure 4.1.3: Sensitivity, $\mathrm{S}$, as a function of the absolute value of the applied electric field. 
Figure 4.1.4: Average time-resolved current transients from detector 4.1.1316.excited with $10-\mathrm{MeV} \mathrm{Si}{ }^{3+}$ ions.

Figure 4.1.5: Stopping power and vacancy concentrations for the interaction of $10 \mathrm{MeV}$ Si ions in diamond as calculațed with TRIM.

Figure 4.1.6: Sensitivity, $S$, as a function of the absolute value of the applied electric field.

Figure 4.2.1: Schematic diagram of the electron-hole plasma created in the detector by stopping of a single incident ion.

Figure 4.2.2: Schematic diagram of a two-dimensional model for the initial charge distribution about a heavy ion track.

Figure 4.2.3: Schematic of a one-dimension approximation to the initial charge distribution about a heavy ion track.

Figure 4.2.4: Schematic diagram of the one dimensional model for charge collection in a RIC detector.

Figure 4.2.5: Distribution of the grid points (totaling 101) along the spatial axis.

Figure 4.3.1: Calculated current transients as a function of the width (FWHM) of the Gaussian distribution of the initial free-carrier density ; the applied electric field was $50 \mathrm{KV} / \mathrm{cm}$.

Figure 4.3.2: Calculated carrier density distribution as a function of space and time; $(A)$ Electron density distribution; (B) hole density distribution.

Figure 4.3.3: Calculated Electric field distribution as a function of space and time.

Figure 4.3.4: Calculated positively charged trap density distribution as a function of space and time.

Figure 4.3.5: Calculated instantaneous current density.

Figure 4.3.6: Temporal variation of the calculated current transient smoothed with a Gaussian function with rise time of $1.3 \mathrm{ps}$ representing ion stopping time 
followed by smoothing with a Gaussian function of representing the impulse response of the recording system with the rise time of $18.6 \mathrm{ps}$.

Figure 4.3.7: Calculated current density output for two different temporal grid widths.

Figure 4.3.8: Temporal variation of the calculated current convolved with the Gaussian functions describing the ion stopping and the recording system response.

Figure 4.3.9: Calculated current transients as a function of applied electric field.

Figure 4.3.10: Comparison between calculated and measured transients for the stopping of $5 \mathrm{MeV} \mathrm{He}{ }^{+}$ions in the detector; A) Applied field is $+50 \mathrm{KV} / \mathrm{cm} . ; \mathrm{B}$ ) Applied field is $-50 \mathrm{KV} / \mathrm{cm}$.

Figure 4.3.11: Comparison between calculated and measured transients for the stopping of $5 \mathrm{MeV} \mathrm{He}+$ ions in the detector; A) Applied field is $+75 \mathrm{KV} / \mathrm{cm}$.; B) Applied field is $-75 \mathrm{KV} / \mathrm{cm}$.

Figure 4.3.12: Comparison between calculated and measured transients for the stopping of $5 \mathrm{MeV} \mathrm{He}+$ ions in the detector; A) Applied field is $+87.5 \mathrm{KV} / \mathrm{cm}$; B) Applied field is $-87.5 \mathrm{KV} / \mathrm{cm}$.

Figure 4.3.13: Comparison between calculated and measured transients for the stopping of $5 \mathrm{MeV} \mathrm{He}{ }^{+}$ions in the detector; A) Applied field is $+100 \mathrm{KV} / \mathrm{cm}$; B) Applied field is $-100 \mathrm{KV} / \mathrm{cm}$.

Figure 4.3.14: Calculated and measured peak currents as a function of electric field with bias polarity being positive.

Figure 4.3.15: Charge collected from ${ }^{241} \mathrm{Am} \alpha$-particles interacting with detector HE6.4 as a function of applied electric field.

Figure 4.3.16: Charge collection efficiency, $\eta$, as a function of applied electric field for HE6.4 derived from excitation with $\alpha$-particles from $241 \mathrm{Am}$. 
Figure 4.3.17: Comparison between measured and calculated response of the detector as a function of track radius with all other computational parameters being constant.

Figure 4.3.18: Comparison between measured and calculated transient currents of the detector with the track radius set at $1.5 \mu \mathrm{m}$.

Figure 4.3.19: Comparison between measured and calculated response of the detector with the track radius set at $1.5 \mu \mathrm{m}$.

Figure 4.3.20: Comparison between calculated and measured transients for the stopping of $10 \mathrm{MeV} \mathrm{Si}^{3+}$ ions in the detector; A) Applied field is $+37.5 \mathrm{KV} / \mathrm{cm}$.; B) Applied field is $-37.5 \mathrm{KV} / \mathrm{cm}$.

Figure 4.3.21: Comparison between calculated and measured transients for the stopping of $10 \mathrm{MeV} \mathrm{Si}{ }^{3+}$ ions in the detector; A) Applied field is $+50 \mathrm{KV} / \mathrm{cm}$.; B) Applied field is $-50 \mathrm{KV} / \mathrm{cm}$.

Figure 4.3.22: Comparison between calculated and measured transients for the stopping of $10 \mathrm{MeV} \mathrm{Si}^{3+}$ ions in the detector; A) Applied field is $+75 \mathrm{KV} / \mathrm{cm}$.; B) Applied field is $-75 \mathrm{KV} / \mathrm{cm}$.

Figure 4.3.23: Comparison between calculated and measured transients for the stopping of $10 \mathrm{MeV} \mathrm{Si}^{3+}$ ions in the detector; A) Applied field is $100 \mathrm{KV} / \mathrm{cm}$.; B) Applied field is $-100 \mathrm{KV} / \mathrm{cm}$.

Figure 4.3.24: Ion track orientation distribution as calculated with TRIM; (A) $10 \mathrm{MeV}$ $\mathrm{Si}$; (B) $5 \mathrm{MeV}$ He.

Figure 4.3.35: Calculated current transient for ions parallel and normal to applied electric field direction.

Figure B.1: Schematic of the arrangement used to measure transient photoconductivity of diamond detectors.

Figure C.1: Schematic of the photoconductor being modeled. 
Figure C.2: Distribution of the total induced charge as a function of initial electronhole pair position and the ratio of carrier mean free path.

Figure C.3: The spatial distribution of induced charge by individual type of carrier at $\beta=1$.

Figure D.1: Schematic of the apparatus used for damaging diamond films.

Figure D.2: Raman/PL spectra as a function of position.

Figure D.3: Spatially-resolved H3 PL peaks across the damaged region of the natural diamond.

Figure D.4: Confocal micrograph of the damaged region from the single crystal, natural type IIa diamond.

Figure D.5: Raman spectra of samples 1 and 3 with fluences of $1.6 \times 10^{12} \mathrm{~cm}^{-2}$ and $1.6 \times 1014 \mathrm{~cm}^{-2}$, respectively.

Figure D.6: Spatially-resolved H3 PL peaks across the damaged regions of sample 3, 4 and 5 with fluences of $1.6 \times 10^{14} \mathrm{~cm}^{-2}, 1.6 \times 10^{14} \mathrm{~cm}^{-2}$ and $1.6 \times 1015 \mathrm{~cm}^{-2}$, respectively.

Figure D.7: Raman/PL spectra of polycrystalline CVD diamond, denoted with a), and single-crystal natural type IIa diamond, denoted with b). The ion fluence for both of the samples is $1.6 \times 10^{14} \mathrm{~cm}^{-2}$.

Figure E.1: Schematic of carrier density distribution in the radial direction. 


\section{List of tables}

Table 1.1.1: Nominal material parameters for typical semiconductors normalized to Si.

Table 2.2.1: Properties of detectors studied in integrated-charge experiment.

Table 2.2.2: List of relevant characteristic times in integrated-charge measurement.

Table 2.2.3: The significant $\alpha$-groups from ${ }^{228} \mathrm{Th}$ source.

Table 2.3.1: List of relevant time scales for time-resolved-current experiments

Table 2.3.2: Characteristics of detectors used in the time-resolved measurements.

Table 3.1.1: Calibrations used to convert channel number to collected charge for the detectors studied.

Table 3.2.1: List of electron and hole mobilities reported by other investigators at 300 K.

Table 3.2.1: Estimated material parameters obtained from fitting the model to the experimental data for all detectors studied.

Table 3.2.2: Estimated material parameters obtained from fitting the model to the experimental data for all detectors studied.

Table 3.2.3: Estimated material parameters obtained from fitting the model to the experimental data for all detectors studied.

Table 3.2.4: Literature values for the average energy required to produce an electronhole pair in diamond.

Table 3.3.1: List of fraction, $f$, used modify the estimated mean values $\mu_{\mathrm{c}}, \tau_{\mathrm{c}}, \mu_{\mathrm{h}}$, and $\tau_{h}$ in calculating fractional resolutions for all three detectors

Table 4.1.1: Experimental conditions used for measurement of current transients.

Table 4.1.2: $\quad$ Pulse parameters as a function of applied bias for detector HE6.4.

Table 4.1.3: Pulse information as a function of bias voltage for detector SI6.9.

Table 4.3.1: Computational parameters used in model calculations for $5 \mathrm{MeV} \mathrm{He}^{+}$-ion excitation. 
Table 4.3.2: Radius of ion track, $r$, and corresponding initial peak carrier density, $n_{o}$.

Table 4.3.3: Comparison of parameters describing the calculated and experimental transients for HE6.4.

Table 4.3.4: Peak carrier density, $n_{o}$, and corresponding ion track radius, $r$, for Si-ion excitation.

Table 4.3.5: Materials parameters used in model calculations for $10 \mathrm{MeV} \mathrm{Si}^{3+}$-ion excitation.

Table D.1: PL peak assignments for the peaks shown in the inset of Fig. D.2. 


\section{Introduction}

In recent years, it has been shown that a solid state detector operating in a mode similar to that of a photoconductor can be used as a fast detector for charged particles [1] and 14-MeV neutrons [2], the latter being detected via nuclear reactions that produce energetic charged particles in the detector. These detectors are conductivity-modulated devices, sometimes referred to as radiation-induced conductivity (RIC) detectors . In actuality RIC detectors are identical in operation to photoconductors that have been studied extensively for many years (see Bube [3] for a general discussion on photoconductors and their operation). The term RIC has been introduced in an attempt to unify the description of the photoconductor response to all types of radiation, such as charged and neutral particles. Such devices are relatively simple in construction. The detector material is sandwiched between two ohmic contacts (this term will be discussed in Chap. 1) and the material's electrical conductivity is modulated by the introduction of either particles or photons.

There are two principle advantages offered by RIC detectors over the pn-junction type of diode detector. The first is that the whole detector volume is made sensitive. The second is the great flexibility in choice of material and its geometry for a specific application. Finally these devices are extremely simple in construction.

Under normal conditions a photoconductor is excited uniformly with photons or, possibly, $\beta$-particles. If the density of the free charge carriers produced is sufficiently small that an applied electric field is not perturbed appreciably, and if the electrical contacts are injecting in character, the current drawn after cessation of excitation will

[1] R. S. Wagner, J. M. Bradley and R. B. Hammond, IEEE Trans. Nucl. Sci., 33(1) (1986) 250.

[2] D. R. Kania, S. M. Lane, B. Jones, C. Bennett, S. G. Prussin and M. S. Derzon, IEEE Trans. Nucl. Sci., 35(1) (1988) 387. 
decay exponentially according to the mean lifetime of the carriers, determined principally by the trapping properties of impurities and lattice defects.

With heavy charged particles (mass equal to or greater than that of a proton), however, energy dissipated through ionization is highly localized in the vicinity of the particle's track. The free carrier density is generally extremely high. Motion of the carriers will lead to a rapid buildup of a space-charge field that can produce large perturbations to the applied field. Thus, the response of a photoconductor to heavy ion excitation can be very different from that normally considered. The need to understand the physics of charge collection under these excitation conditions has been the motivating factor for the research for this dissertation. In order to investigate the issues associated with the charge collection, we have chosen to investigate the excitation of diamond with $\alpha$-particles from radioactive decay, and with $\mathrm{He}^{+}$and $\mathrm{Si}^{3+}$ ions produced with a small particle accelerator.

Diamond has a number of properties that make it an interesting material for radiation detection under certain conditions. First, it has a large bandgap of about $5.5 \mathrm{eV}$, and thus exhibits a very low dark current at normal temperatures without the need to form a pn-junction, as is the case materials like Si. This means that room- and possibly elevated-temperature applications may be possible. Second, diamond also has one of the lowest known dielectric constants. This means that detectors of small capacitance can be constructed, and they will tend to be inherently fast. Third, because of the higher binding energy of a carbon atom in diamond as compared to a Si atom in normal silicon, the radiation resistance of a diamond detector should be significantly higher than available with current $\mathrm{Si}$ technology. Finally, with the advent of large area, thin films grown by chemical vapor deposition (CVD) techniques, this material is potentially important for a wide variety of electronic device applications.

To study the physical processes of charge collection in diamond under local excitation conditions, we first need to understand some aspects of carrier transport 
properties in the material. To gain this, we have developed a simple experimental arrangement to measure charge collection from diamond detectors, and a transport model with which estimates of such properties as the carrier mobilities and lifetimes, and the mean energy required to produce an ion pair can be obtained. A windfall from this work is that the experimental arrangement along with the model provides a simple, and relatively rapid screening method for assessing the quality of various diamond materials for various applications . 


\section{Acknowledgments}

It is quiet now on College Avenue with cars passing intermittently. Every now and then, a bus roars by the apartment causing the whole building to rumble with such force as to cause my coffee cup to chatter against its saucer like the state of my nerves.

"Three a.m., Dufess. That's the three a.m."

Dufess remained silent. Dufess, an eighteen-year-old canine, had not pricked up his ears as dogs are supposed to do when an event out of ordinary occurs. I use the term canine because I and a bevy of friends couldn't fathom what breed or combination of breeds Dufess might be. Typically, our discussions started out logically and enthusiastically, but would end awkwardly, as if we had both wished that I had not brought up the subject.

"That's a Greyhound body," one friend said, "but his head is too big. Maybe he's an underfed Doberman. Bit his snout's too large. He's old, so maybe his shape has changed. Does he eat much? If he had a fuller body, I might think of him almost as a shepherd, you know, a German Shepherd. If it wasn't for his fur..."

The fur. I think that is what upset the logic of all observers the most as to Dufess's make-up. His fur was thick, tightly curled, with a brownish-white color, but curled to the point of having a matted quality. This fur was constantly wet and tight against his skin, like a marathon runner during his last mile or two. To make matters worse, he was constantly shivering. Indeed, by those who did not know my basic nature, I was accused of having exercised this poor mutt in his waning days to a terminal degree.

"Look," I'd respond, "first of all, I'm only looking after this dog for a friend, as he doesn't get much exercise except when I've got the time. These days, with this albatross around my neck, that means at the most may be two walks a day. If he's lucky. Otherwise, his activities are his own because I'm hardly ever home. As far as I know, he escapes out my second-story window and takes wing around Berkeley." 
And that little speech would bring on an embarrassing silence to be followed by a quick change of subject.

"Three a.m., Dufess. That's the ever so familiar three a.m.," I exclaimed, "all I have to do now are the Acknowledgments. That's all I have to do and this helium-filled balloon exercise will be finished. That's it. That's all I have to do."

Dufess looked up at me, more because I was looking at him I think, than anything I had actually said.

I sat staring at my computer screen, at the words that had become my life, at words that had become greater and deeper than any relationship I'd ever known. I looked and looked and waited for the finale to come to my fingertips and transform into words. My fingertips, in turn, waited for me close this relationship. They waited desperately for an end to the hours that turned into days that turned into weeks that turned into months. There was sweat and blood on these hundreds of pages. It was madness, and to end it all, all I had to do were the Acknowledgments. But I had hit a wall.

"Do you know what you and I have in common, Dufess?" I said offhandedly, "We're both dying."

"You'll be okay. In a short period, you'll be a scientist."

When I heard those words, and when in the picoseconds that passed I determined that no one else had entered my apartment and that the answering machine had not turned on and that the TV and stereo were off, I responded with an empirical, "Christ!" and turned to face Dufess.

"You'll be okay." he repeated, "By sun-up I'll be dead and you'll have finished your Acknowledgments."

"Christ!" I repeated.

"Hardly," Dufess said sardonically, "But like Christ, you've got to know when it's time to let go. You've got to finish this thing. There's a life ahead of you and it can't start until you acknowledge those who got you there to begin with."

xxiii 
"What do you know about it?" I asked. There was amazement, skepticism and doubt in my voice. After all, I figured, when it comes to a thesis, anything is possible, but what would a dog know?

“Nothing. But I know that I wouldn't be here without a long list of those who helped me."

"But Dufess, where do I start? I'm still lost on list of people who should be acknowledge, first! What if I forget to mention someone important!"

In these waning moments of frenzy, I realized that the Acknowledgments are just the culmination of sweat and blood that stained this written parody. As this realization progressed, frenzy turned into mere nervousness and then turned into calmness. At this precise moment, the words to thank those people who helped to cope with this seemingly unending relationship started to flow.

"Well Dufess, here it goes."

"There have been many people over the years who have given me the encouragement and the push that I needed. I would first like to thank my family for their persistent pressure and their refusal to accept my excuses. Without them, I could not have finished this work. Of course, my mother gets the special thanks. Overflowing appreciation goes to Barbara Zappe, my close friend, who gave me the strength and the unending support during the times of low motivation and disappointments. I would, also, like to thank Zappe and Johnson families for their contributions in sustaining my voracious appetite for food. My close friends Dave Williams, Dean and Maggie RussellHarde, Mike Johnson, Hector Beguirstain and Debra Marchevsky receive the gratitude award for waiving their "visitation rights" until the end of this dissertation.

I also would like to thank my research advisers Dr. Don Kania and Dr. Steve Lane who encouraged me to excel, to think fundamentally and to question. Of course, I can't forget my research colleague and a friend Dr. Lawrence Pan for fun and games during our late nights of work, long discussions and jokes. Warm thanks go to Dr. Joel Ager for his 
help in finishing the radiation-damage part of this dissertation. Also, the technical staffs of LEAD at Lawrence Livermore National Laboratory, especially Perry Bell, Joe Sartariano and Greg Hensley for their patience while I learned to machine and to build circuits, the microfabrication laboratory staff, especially Dave and Debra Hebert, of UC Berkeley for their patience while I learned to fabricate the detectors used in this dissertation, and IBML of Los Alamos National Laboratory, especially Caleb Evans, Mark Hollander, Dr. Joe Tesmer and Dr. Carl Majiore for letting me overrun my beam time allotments. I would, further, like to thank Dr. Ron Wagner of Los Alamos National Laboratory for stimulating discussions in the areas of high-speed signal transmission and device fabrication and for the use of his apparatus.

Finally, I can't thank my academic and research adviser Prof. Stanley G. Prussin enough for giving me the chance to do when and what I wished, for letting me fall on my face when appropriate, and for picking me up after the fall. I'll always be grateful for all of his encouragements and understanding.

For those whom I have failed to mention due to my absent-mindedness, I extend my deepest gratitude."

"My God Dufess, I think I'm finally done," I exclaimed! But as the sun came up to start another glorious day, Dufess didn't answer... 


\title{
Chapter 1: Theory of radiation-induced conductivity
}

\author{
1.1 Brief history and some general information on the use of diamonds for radiation \\ detection
}

In the period 1920-50, interest in using diamond for other than decorative and mechanical purposes became apparent. Gudden and Pohl [1] prepared one of the first diamond detectors and used it with uniform illumination by light for photoconductivity experiments. McKay [2] and Hofstadter [3] used a similar device to detect $\alpha$ - and $\beta-$ particles. These applications were due, in part, to the greater availability of large singlecrystals of natural diamond that were "pure" enough as compared to other detector materials, such as silicon and germanium. Unfortunately, because of severe polarization effects, which is essentially a formation of space-charge region in the detector usually resulting from the trapping of carriers, possible rectifying nature of the electrical contacts used, and the prohibitive cost of the material, general applications as detectors could not be approached. During the period 1950-60, interest in the response of natural diamond to radiation became quite intense $[4,5,6,7,8]$. Again, these efforts were plagued by the polarization problem .

In the early 1970's , Kozlov and Konorova [9] fabricated diamond particle detectors without any apparent polarization effects by applying platinum contacts followed by heat treatment. Finally, in the early 1980 's, Moazed, et al. [10] reported the first preparation of an ohmic contact on diamond. This was an important technological advance from the standpoint of electronic device fabrication, including the application to radiation detection. With the recent advent of chemical vapor deposited diamond[11], and the rapid improvement in both the dimensions of the prepared material and its electrical properties [12], room temperature diamond detectors are now within reach.

It is natural to ask "why is diamond so attractive as a detector or electronic 
material?" To answer this in principle, the carrier saturation velocity $\left(v_{\text {sat }}\right)$, dielectric breakdown field $\left(\mathrm{E}_{\mathrm{BD}}\right)$, thermal conductivity $(\mathrm{K})$, and dielectric constant $(\varepsilon)$ of several narrow- and wide-bandgap semiconductors important in radiation detectors and electronic devices, normalized to the corresponding values of silicon are listed in Table 1.1.1. Inspection of the table shows that diamond has superior properties in comparison to Si and may have the best properties of all common semiconductor materials considered. In particular, the large carrier saturation velocity, the highest dielectric breakdown field, the highest thermal conductivity, and the smallest dielectric constant compared to other semiconductors, is considered ideal for high-speed and high-power applications, as well as for radiation detection.

Table 1.1.1: Nominal material parameters for typical semiconductors normalized to Si. [12]

\begin{tabular}{|ccccc|}
\hline Material & $v_{\text {sat }}$ & $\mathrm{E}_{\mathrm{BD}}$ & $\mathrm{K}$ & $\varepsilon$ \\
\hline $\mathrm{Si}$ & 1.0 & 1 & 1 & 1 \\
$\mathrm{Ge}$ & 2.5 & 0.3 & 0.4 & 1.3 \\
$\mathrm{GaAs}$ & 1.5 & 1.3 & 0.3 & 1.1 \\
$\mathrm{SiC}$ & 1 & 2.5 & 3.3 & 0.6 \\
Diamond & 2 & 33.3 & 13.3 & 0.5 \\
\hline
\end{tabular}

In performing the research work presented in this dissertation, we used singlecrystal, natural diamond specimens which belong to a class of diamond called type IIa. This type of diamond is known to be electrically insulating with a nominal, singlydispersed, paramagnetic nitrogen concentration of $20 \mathrm{ppm}$. It is known to be rare in nature and has a semiconducting cousin which is even rarer called type IIb. This is the only type of diamond known to be naturally semiconducting with the major impurity being boron with a nominal resistivity of $10^{4} \Omega-\mathrm{cm}$. The remaining types are Ia and $\mathrm{Ib}$ 
which are known to contain roughly parts per ten thousand to parts per thousand of nitrogen in cluster or singly-dispersed form, respectively. A detailed description of the characteristics of these different types of diamond can be found in Ref. 13.

\subsection{Radiation interaction with matter}

All radiation, whether charged particles, photons, neutrons, etc., interact as they traverse matter. While charged particles interact directly by their Coulomb fields, neutral particles depend on different properties. Photons, including the full spectrum of ionizing electromagnetic radiation, interact primarily, by the photoelectric effect, Compton scattering, and pair production. Also possible, but much less common, are photonuclear reactions, for example, $(\gamma, \mathrm{n})$. Neutrons interact only via the strong interaction, and can be detected via those nuclear reactions that directly or indirectly lead to the production of charged particles. Discussions on all of these interactions can be found in any textbook on nuclear physics [14].

The interaction between the incident radiation or subsequent charged particles and target atoms in some medium results in a transfer of energy. This energy appears in various forms: electronic excitation of atoms, ionization of atoms, molecular excitations, etc. Electrons produced directly or immediately after a primary interaction, as well as recoil atoms from elastic and inelastic scattering, typically have very high kinetic energies relative to thermal. Through additional interactions, these will thermalize quickly, with creation of additional excitations and ionizations, etc.

With respect to radiation detection, it is the total ionization produced by the cascade of interactions following interaction of radiation in matter that is the most useful. The movement of ionized atoms and free electrons in a gas, or the movement of free electrons and holes in a solid, under the influence of an applied electric field results in the generation of a current pulse that can be easily detected and whose properties, under 
certain conditions, can be measured to define the energy deposited in the detector, the type of particle that interacted, the time at which the interaction occurred, etc. The interactions which are described above have been studied extensively $[15,16,17,18,19$, $20,21,22,23,24,25,26,27,28,29]$.

For interactions in semiconductors, it is important to recognize that elastic and inelastic scattering of incident radiation, especially heavy charged particles, on atoms of the host can cause production of vacancies, interstitials, and other types of lattice defects. Any disruption of periodicity in a semiconductor crystal can create trapping sites for carriers, and in the majority of cases, structural defects appear as both deep and shallow traps $[14,15]$. Some of the disruptions due to radiation may be reversible. For example, structural defects produced in a solid by electron bombardment can be annealed out by heating the solid to elevated temperatures, causing migration of interstitials and vacancies and their subsequent annihilation $[14,15]$.

Unfortunately, a quantitative understanding of radiation damage effects on the electrical characteristics of diamond is not presently known. One of the main defect centers that can be formed by irradiation with energetic particles is known as $\mathrm{H} 3$ and is known to be di-nitrogen-vacancy complex [30]. This defect is found in small concentrations in unirradiated diamonds (see appendix D), also. The electrical behavior of this defect, such as whether or not the center acts as a recombination site, is not known. Also, it has been calculated that a large fraction of the vacancies produced through irradiation exists in the negative charge state after capturing electrons [31]. Another importance defect center known in undamaged and radiation-damaged diamond is the GR center. This center is known to be a vacancy where the defect is speculated to exist as a neutral vacancy [32]. Therefore, it appears that vacancies may play an important role in the trapping/recombination dynamics of free carriers.

Because of the much larger lattice binding energy of carbon in diamond (atomic displacement energy, $E_{d}=43 \mathrm{eV}[33]$ ) as compared to a silicon atom in normal silicon 
$\left(E_{d}=25 \mathrm{eV}[15]\right)$ it is expected that diamond will be considerably more radiation resistant than silicon. But nonetheless, damage will occur in diamond as well. Then, one critical question is: can radiation damage be annealed in diamond so that the near pristine crystal can be restored by heating the crystal at elevated temperatures similar to the annealing processes used for radiation-damaged silicon to recover much of the original condition of the crystal lattice? It has been speculated [34] that interstitials may be extremely mobile within the lattice even at room temperature but vacancies may not be. Han, et. al. [35] (see appendix D) and Adel, et al. [36] suggest that even vacancies may be quite mobile at elevated and room temperatures. Therefore, one of the main prerequisites for annealing out of radiation damage at elevated temperatures may exist, already. But, it is not clear whether the broken bonds surrounding the damaged region establish themselves as $\mathrm{sp}^{2}$ (graphite) or $\mathrm{sp}^{3}$-hybridized (diamond) bonds when the damaged crystal is heated to an elevated temperature. Some investigators have speculated that the re-establishment of $\mathrm{sp}^{3}$-hybridized bonds can be accomplished through annealing at elevated temperatures to a certain extent [37] and Parikh, et. al. [38] have implied that some fraction of radiationdamage can be annealed by heating the lattice to $950^{\circ} \mathrm{C}$ in vacuum for 1 hour.

\subsection{Radiation-induced conductivity (RIC)}

The term radiation-induced conductivity (RIC) can be considered to have its origin in the term introduced by Vul [39] in 1961. At that time the term was referred to as radiation conductivity analogous to photoconductivity in an attempt to unify the effects of all forms of ionizing radiation, such as charged and neutral particles, $\mathrm{x}$-rays, $\boldsymbol{\gamma}$-rays, etc., on the conductivity of semiconductors. With respect to the phenomenon of photoconductivity, the theoretical structure has been extensively studied and reviewed by Bube [40]. Therefore, essentially the entire theoretical framework developed to describe 
photoconductivity can be applied directly to the case of RIC to describe the dynamics of the non-equilibrium density of free carriers produced by incident radiation.

Any interaction of radiation with the semiconductor will produce free electrons and holes that will increase the conductivity of the material and exhibit a characteristic lifetimes defined by the rates of direct recombination and trapping, and the rate at which the carriers are swept out of the detector under the influence of an applied electric field.

The total conductivity is expressed by

$$
\sigma_{o}+\Delta \sigma=e\left(\mu_{n}\left(n_{o}+\Delta n\right)+\mu_{p}\left(p_{o}+\Delta p\right)\right)
$$

where $\sigma_{\mathrm{o}}=$ thermal equilibrium conductivity

$$
\begin{aligned}
& \Delta \sigma=\text { change in conductivity due to the incident radiation } \\
& \Delta \mathrm{n}=\text { excess free electron density due to the incident radiation } \\
& \Delta \mathrm{p}=\text { excess free hole density due to the incident radiation } \\
& \mathrm{n}_{\mathrm{o}}=\text { thermal equilibrium density of free electrons } \\
& p_{\mathrm{o}}=\text { thermal equilibrium density of free holes } \\
& \mathrm{e}=\text { elementary charge } \\
& \mu_{\mathrm{n}}=\text { hole mobility } \\
& \mu_{\mathrm{p}}=\text { electron mobility }
\end{aligned}
$$

Estimation of the thermal equilibrium conductivity can be obtained with use of statistical considerations as outlined in introductory semiconductor physics texts [41, 42]. From practical considerations, thermal generation can be neglected in the case of diamond due to its large band gap of $5.48 \mathrm{eV}$. Then Equ. 1.3 .1 can be reduced to

$$
\Delta \sigma=e\left(\mu_{n} \Delta n+\mu_{p} \Delta p\right)
$$

It is obvious from Equ. 1.3.2 that the temporal and spatial dependence of the change in conductivity results from the temporal and spatial variation in the excess free electron and hole densities produced by the incident radiation. Under steady state external excitation, 
Equ. 1.3.2 can also be expressed in terms of the lifetime of the free carriers and the freecarrier generation rate , i.e.,

$$
G=\frac{\Delta n}{\tau_{n}}=\frac{\Delta p}{\tau_{p}}
$$

where $\mathrm{G}=$ free carrier generation rate, usually quoted as (electron-hole

$$
\begin{gathered}
\text { pairs) } / \mathrm{cm}^{3} \text {-sec } \\
\tau_{n}=\text { free electron lifetime, sec } \\
\tau_{p}=\text { free hole lifetime, sec }
\end{gathered}
$$

Upon substituting Equ. 1.3.3. into Equ. 1.3.2, we obtain

$$
\Delta \sigma=e G\left(\mu_{n} \tau_{n}+\mu_{p} \tau_{p}\right)
$$

It is clear that in a pure semiconductor, the lifetimes of the two types of carriers are equal since electrons or holes are free until they recombine. From Equ. 1.3.4, we can also see that the performance of a RIC device depends on the efficiency of the carrier generation, the carrier lifetime, and the carrier mobility.

In all real materials there are always some impurity atoms or lattice defects, referred to in the following simply as defects. Neglecting those that are thermally-excited donors or acceptors, defects can be ionized via photons and thereby cause an increase in the number of free electrons or holes in a material, resulting in greater conductivity. This is referred to as extrinsic photoconduction. The photon energy required for this to occur is less than the band gap of the intrinsic material. Thus, a defect with ionization energy less than the band gap can give rise to an additional conduction electron and a fixed hole. Similarly, a defect may capture an electron from the valence band creating an additional mobile hole and a fixed negative charge at some point in the lattice. The effective energy levels corresponding to such defects can exist anywhere within the band gap. The depth of the defect levels, or energy relative to the bottom of the conduction band in the intrinsic material determines whether a defect represents a deep center (or recombination 
center) or a shallow center. The nature of such centers is discussed further in section 1.3.2.

Extrinsic effects on conduction are more pronounced at lower temperatures, especially below temperatures at which band-to-band transitions are small. Since the energy difference between some defect levels and conduction and valence bands can be quite small, i.e. on the order of $\mathrm{kT}$ or less, the transition of trapped carriers from defect energy levels to the conduction or valence bands can be made quite easily.

\subsection{Principles of detector operation}

With the discussion in Sec. 1.3, the operation of RIC devices as radiation detectors is easily explained. A simple schematic of a detection system based on such a device is shown in Fig. 1.3.1. As mentioned in the introduction, these devices are relatively simple and are made by placing the detector material between two ohmic contacts. As the incident radiation interacts with detector material, excess carriers are formed by ionization in the bulk. These carriers drift under the influence of an applied electric field and thereby induce current at the contacts. The magnitude of the current density, assuming that Ohm's law is obeyed, can be written

$$
J=|\vec{E}| \sigma
$$

where $\mathrm{J}=$ current density

$$
|\vec{E}|=\text { applied electric field strength }
$$

The change in current density due to the conductivity modulation resulting from incident radiation as calculated in Sec. 1.3 can be written as

$$
\Delta J=|\vec{E}| \Delta \sigma
$$




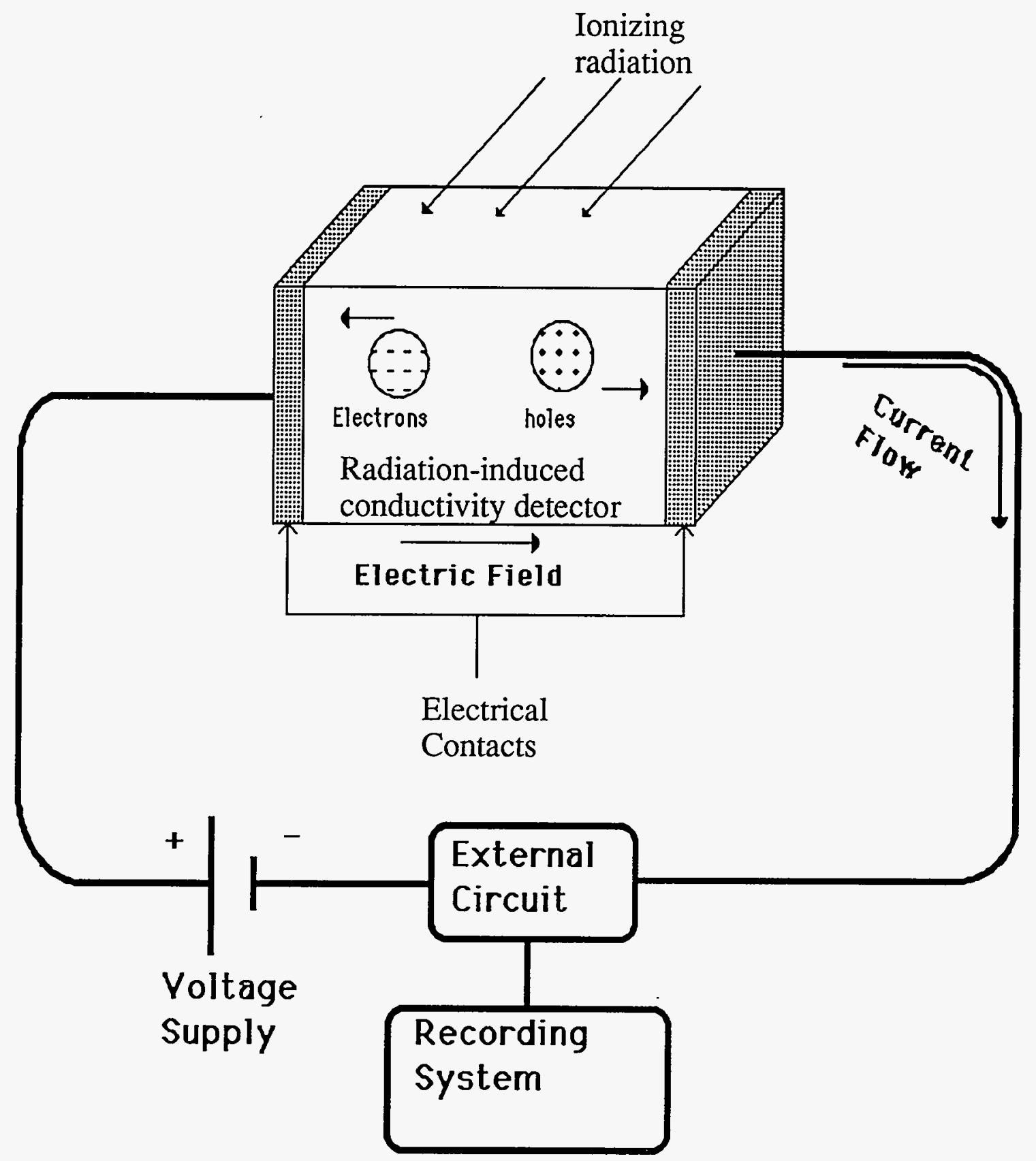

Figure 1.3.1: Schematic of a general radiation detection system 
It is clear, then, that the temporal variation in the change in current density will arise from the temporal variation in the conductivity if the applied field is constant. Should highspeed operation be desired, the temporal variation in the change in conductivity must be correspondingly rapid. This implies that the temporal dependence of the current that can be drawn from the detector is actually a function of the time variation of the excess carrier densities (see Equ. 1.3.4). Therefore, if the lifetime of the excess carriers were made short enough, any temporal variation in the intensity of the exciting radiation would be reflected in the temporal variation of the conductivity and hence in the current.

For the specific case of steady state operation, Equ. 1.4.2 can be written as

$$
\Delta J=e|\vec{E}| G\left(\mu_{n} \tau_{n}+\mu_{p} \tau_{p}\right)
$$

by substituting for $\Delta \sigma$ from Equ. 1.3.4. As long as the carriers drift under the influence of the electric field, a detector will continue to draw current.

In response to the introduction of localized carrier pairs, the applied electric field will tend to cause the system to relax, i.e., to transform from this high-energy excited configuration to the quiescent state with no free charge carriers, by several mechanisms. Because these are of especial interest to high-speed response, these mechanisms are discussed in some detail in the following.

\subsubsection{Dielectric Relaxation}

Dielectric relaxation is one of the mechanisms by which a material reacts to a net charge distribution to bring about charge neutrality. If there are mobile charges present, then these charges will move in the direction to maintain charge neutrality as whole. Thus, in a semiconductor the charges contributed by the free electrons, the free holes, and the ionized impurity atoms must neutralize each other, on the average.

As injection of excess charge carriers in the material occurs, they begin to move. These moving charges will create a local self-field. The material will react to reduce the 
self field to zero, on the average, in a characteristic time known as the dielectric relaxation time.

From electromagnetic theory, the local electric field due to an excess charge density, $\rho_{e}=\rho-\rho_{0}$ where $\rho_{0}=$ equilibrium charge density, is given by Gauss' law,

$$
\int \vec{E} \bullet d \vec{S}=\left(\frac{1}{\varepsilon}\right) \sum q_{i}
$$

$$
\begin{aligned}
& \text { where } \varepsilon=\text { permittivity }=\varepsilon_{\mathrm{r}} \varepsilon_{\mathrm{o}} \\
& \varepsilon_{\mathrm{T}}=\text { material permittivity } \\
& \varepsilon_{\mathrm{o}}=\text { permittivity in vacuum } \\
& \mathrm{q}_{\mathrm{i}}=\text { the total net charge enclosed by the surface } S .
\end{aligned}
$$

This can be also written in terms of the displacement vector, $\vec{D}$, as

$$
\int \vec{D} \cdot d \vec{S}=\sum q_{i}
$$

Furthermore, the integral can be expressed in terms of the excess charge enclosed in a closed volume, i.e.,

$$
\int \rho_{e} d V=\int \vec{D} \cdot d \bar{S},
$$

or, writing the integral in differential form, we have

$$
\vec{\nabla} \bullet \vec{D}=\rho_{e}
$$

This is just one form of Poisson's equation. In one dimension,

$$
\frac{\partial E}{\partial x}=\frac{e \rho_{e}}{\varepsilon} .
$$

The one-dimensional continuity equation for charge carriers is given by 


$$
\frac{\partial \rho}{\partial t}+\frac{1}{e} \frac{\partial J}{\partial x}=0
$$

The current density equation is given by

$$
J=(\sigma E)-e D_{\rho} \frac{\partial \rho_{e}}{\partial x}
$$

where $\sigma=$ conductivity

$$
\mathrm{D}_{\rho}=\text { charge diffusivity }
$$

Differentiating Equ. 1.4.7 with respect to $\mathrm{x}$ and inserting Equ. 1.4.5, we obtain

$$
\frac{1}{e} \frac{\partial J}{\partial x}=\frac{\sigma \rho_{e}}{\varepsilon}-D_{\rho} \frac{\partial^{2} \rho_{e}}{\partial x^{2}}
$$

Substituting Equ. 1.4.7 into Equ. 1.4.8, we finally obtain

$$
\frac{\partial \rho_{e}}{\partial t}+\frac{\sigma \rho_{e}}{\varepsilon}-D_{\rho} \frac{\partial^{2} \rho_{e}}{\partial x^{2}}=0
$$

This equation can be solved by separation of variables [34], and the solution to the temporal equation is

$$
\rho_{e}=\rho_{e o} \exp \left(-\frac{t}{\tau_{d}}\right),
$$

where $\tau_{d}=\varepsilon / \sigma$,

and $\tau_{\mathrm{d}}$ is called the dielectric relaxation time.

The dielectric relaxation time can be visualized from the following considerations. Given some initial excess charge distribution in a medium, the charge will tend to redistribute itself as a result of the electric field due to the charges and any external field that may be applied. The characteristic time for the charge to redistribute and attain an equilibrium distribution is just the dielectric relaxation time. 


\subsubsection{Relaxation by carrier recombination and trapping.}

If carrier pairs are created in an ideal semiconductor free of defects and impurities, the only mechanism for recombining of the carriers is by band-to-band transitions into the vacant states created by the formation of the carriers themselves. These vacant states are usually represented by holes. Depending upon the material properties, two types of transitions can exist. For materials such as GaAs, the conduction band level with the lowest energy of characteristic wave vector $\mathbf{k}=0$ lies directly above the level in the valence band with the highest energy, also corresponding to $k=0$ (see Fig. 1.3.1). In such materials, recombination takes place via these levels and the material is said to relax by direct recombination.

For some materials, such as silicon or diamond, the level in the conduction band with minimum energy does not correspond to $\mathbf{k}=0$, as shown in Fig. 1.3.2., but lies some $\Delta \mathbf{k}$ away from it. Electrons excited at levels corresponding to $\mathbf{k}=0$ will, as a result of lattice vibrations, rapidly populate levels in the second potential well shown in Fig. 1.3.2, and recombine by transitions to vacancies in the valence band. Because this process is rapid, this indirect path of recombination will tend to dominate and the resultant recombination is referred to as indirect recombination. Regardless of the dominant mode, recombination rates in such ideal materials are slow, and carrier lifetimes as long as $1 \mathrm{sec}$ can be expected [48]. In reality, this is seldom found because of the presence of impurities or structural defects.

Impurities generally introduce energy levels within the forbidden gap of the ideal material and the presence of these levels provides an additional mechanism for recombination and/or trapping of free carriers. On a gross scale, the levels due to impurities can be classified as shallow or deep. Shallow levels lie close to the band edges and can be either donors or acceptors to the lattice. Frequently, the energy difference between the band edge and the energies of shallow levels is on the order of kT. If a 


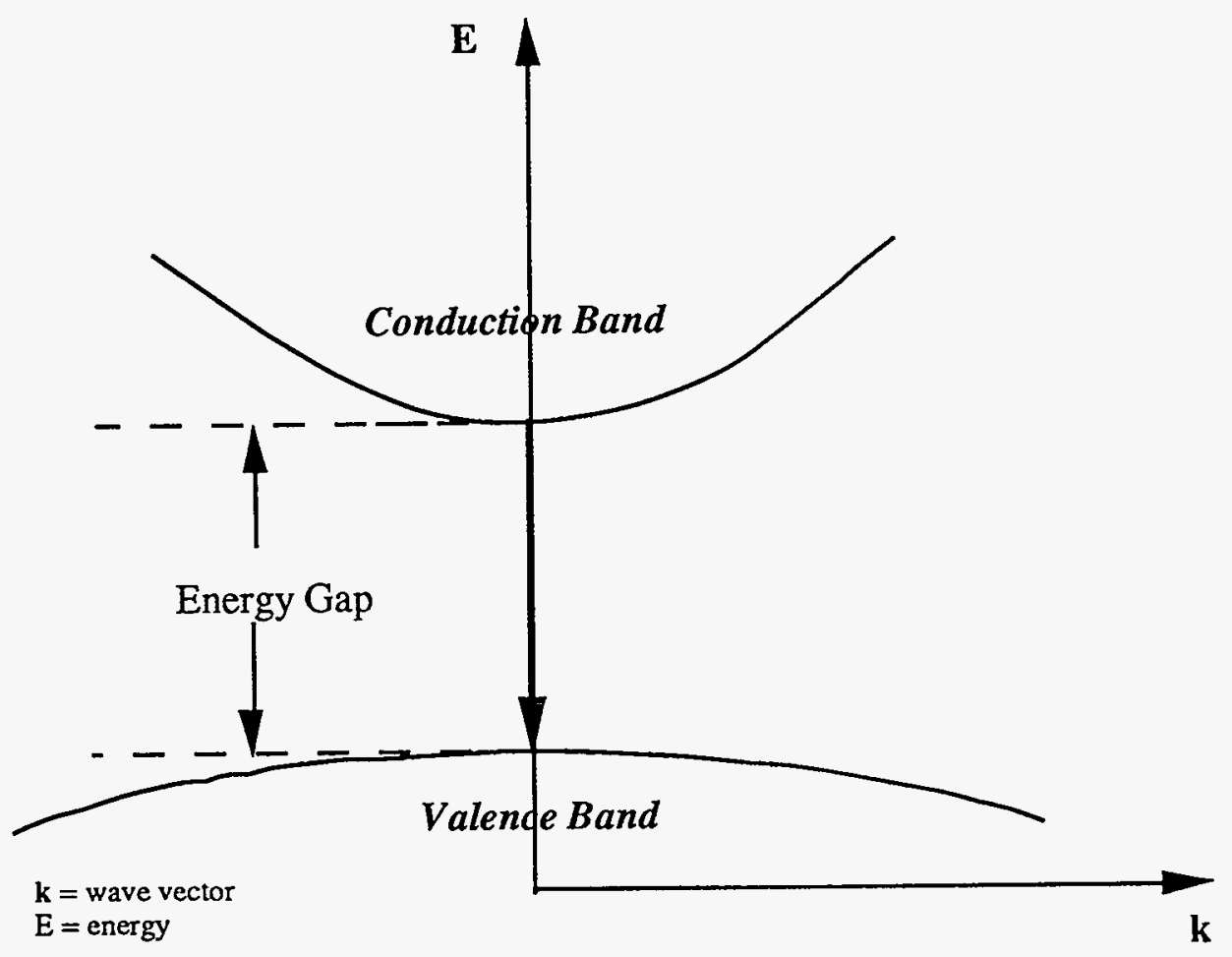

Figure 1.3.1: Energy valley representation of the direct band-to-band recombination. 


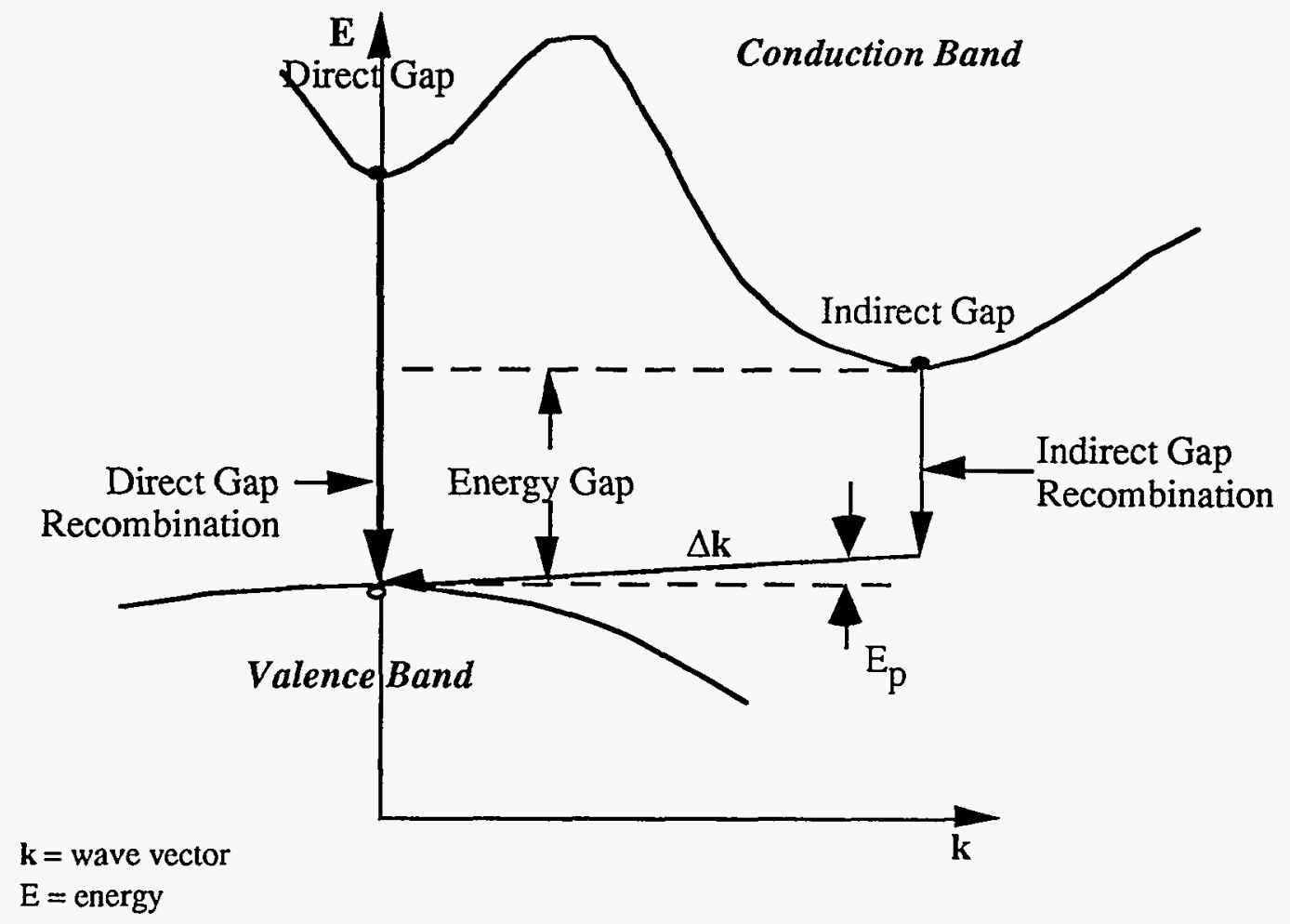

Figure 1.3.2: Energy valley representation of the indirect band-to-band recombination. This transition is usually aided by emission of a phonon of energy $\mathrm{E}_{\mathrm{p}}$.

carrier falls into one of these levels, there is a high probability of it being re-emitted due to thermal excitation. The time spent by free carriers in shallow levels can vary over a large range and times as short as a few nanoseconds and as long as a few milliseconds are common [43]. While residing in a shallow level, or shallow trap, there can be an enhanced probability for recombination to occur and thus the presence of shallow traps can produce a significant reduction in carrier lifetimes. This type of recombination is referred to simply as trap-assisted recombination. With respect to diamond, there are no known impurities that can be considered to introduce shallow levels in a traditional sense, i.e., that introduce levels within a few $\mathrm{kT}$ of a band edge. For example, boron impurity in diamond is considered to be "shallow" acceptor, but it introduces an energy level at 
about 365-374 meV [44] above the valence band which is much more than few kT away from the valence band edge. Nevertheless it is completely possible that structural defects may introduce levels which can be considered shallow.

Deep levels are energy levels introduced by impurities or other defects that lie far away from the band edges. In natural diamond, one of the most common impurities that introduces deep levels is nitrogen. It introduces a level at approximately $2 \mathrm{eV}$ below the conduction band for substitutional nitrogen which is the form seen in type Ila diamond.

The time spent by free carriers in deep levels can be as short as a few milliseconds or as long as seconds depending on the depth of the level [45]. Due to this large residence time, deep levels tend to be efficient recombination centers [46]. On the time scales relevant to applications of fast-response detectors, perhaps 1-100 ps, the capture of a carrier in a deep trap as shown schematically in Fig. 1.4.3 essentially removes it from contributing to current flow and charge collection.

Conduction Band

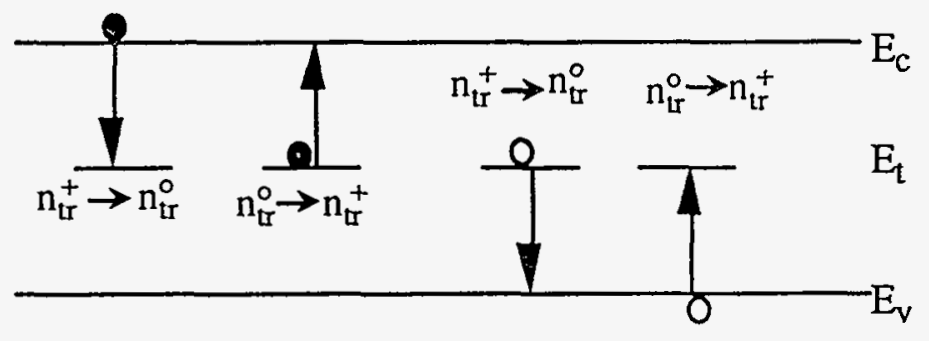

Valence Band
(A)
(B)
(C)
(D)

Figure 1.4.3: Four possible level assisted processes:
A) electron capture from the conduction band to an ionized level
B) electron emission from a neutral level to the conduction band
C) hole emission by an ionized level
D) hole capture by a neutral level 
Besides an impurity such as nitrogen in diamond, there are other sources of deep levels in semiconductors that increase trapping and the rate of recombination of carriers. These sources can be structural defects such as dislocations, vacancies, and interstitials, etc. In addition, these defects can be artificially produced through the bombardment of semiconductors with high-energy particles $[13,14]$.

As an illustration of the means for quantifying the effects of deep traps on the response of a detector to ionizing radiation, we consider the important case of nitrogen in natural type IIA diamond. As indicated earlier, nitrogen can act as a donor to diamond and is considered to exist in the two charge states 0 and +1 . Suppose that the total concentration of the nitrogen is $\mathrm{N}_{\mathrm{tr}}$. Using the Fermi-Dirac distribution [47] and in the absence of carriers other that those generated thermally, the probability that a nitrogen atom has donated an electron can be written

$$
\frac{n_{t r}^{+}}{N_{t r}}=\frac{1}{1+\exp \left(\frac{\left(E_{t r}-E_{f}\right)}{k T}\right)},
$$

where $n_{t r}^{+}=$density of positively-charged sites, $\mathrm{cm}^{-3}$

$$
\begin{aligned}
& E_{\mathrm{tr}}=\text { the level energy represented by the nitrogen impurity, eV } \\
& E_{\mathrm{f}}=\text { Fermi level, eV, }
\end{aligned}
$$

and the density of neutral sites is then given by $n_{t r}^{\circ}=\left(N_{t r}-n_{t r}^{+}\right)$.

If free electrons are introduced at a density $n$, electrons will be removed by trapping at the positively-charged sites and the rate of their removal at any point in the material can be written as

$$
-\frac{\partial n}{\partial t}=B_{n} n n_{t r}^{+}
$$

where $B_{n}=$ rate constant, $\mathrm{cm}^{3} / \mathrm{sec}$ 
Since holes can be captured by negatively charged levels, and holes, in turn, recombine with the trapped electrons, we can write a similar equation for the rate of loss of holes due to the levels as,

$$
-\frac{\partial p}{\partial t}=B_{p} p n_{t r}^{\circ}
$$

where $\mathrm{p}=$ density of holes, and $\mathrm{B}_{\mathrm{p}}=$ rate constant, $\mathrm{cm}^{3} / \mathrm{sec}$

In the general case, the densities of free carriers vary both in time and space, and thus, the functions $n$ and $p$ should be expressed as $n(r, t)$ and $p(r, t)$ where $r$ is the spatial vector describing the position of the volume under consideration.

The rate constants $B_{n}$ and $B_{p}$ are related to the electron and hole capture cross sections on deep levels, and the electron and hole thermal velocities. Thus, the rate constants are defined as

$$
\begin{aligned}
& B_{n}=\left\langle v_{n} \sigma_{n}\right\rangle \\
& B_{p}=\left\langle v_{p} \sigma_{p}>\right.
\end{aligned}
$$

where $v_{n}=$ electron thermal velocity, $\mathrm{cm} / \mathrm{sec}$

$$
\begin{aligned}
& v_{p}=\text { hole thermal velocity, } \mathrm{cm} / \mathrm{sec} \\
& \sigma_{n}=\text { electron capture cross section, } \mathrm{cm}^{2} \\
& \sigma_{p}=\text { hole capture cross section, } \mathrm{cm}^{2} \\
& <>=\text { signifies average value }
\end{aligned}
$$

Even though capture cross sections are the fundamental quantities, they are generally unknown.

\subsection{Electrical contacts to diamond}

For electronic applications, contacts to a material are important since any active device performance is directly related to the quality of the contacts. A variety of metals 
have been reported to form both ohmic and Schottky contacts to diamond. Care must be taken in interpreting the reported data, because both electrons and holes are mobile, and a contact that is ohmic to one carrier is generally blocking to the other. This is not an issue in doped diamond, but must be considered for intrinsic diamond.

\subsubsection{Ohmic contacts}

An ohmic contact can be defined as one that has small resistance compared to the bulk series resistance of a semiconductor or an insulator to which it is affixed, i.e., in the presence of an applied electric field, the voltage drop across the contact is negligible in comparison with the voltage drop across the bulk material. Frequently, however, the term "ohmic contact" is used to describe any contact/material junction that possesses a linear I-V characteristic. This usage, while having obvious utility, is quite ambiguous, since contacts that are "ohmic" to one carrier type are often are not "ohmic" to the other.

For the present purposes it is important to distinguish not only between contacts that display linear I-V characteristics, but also to distinguish contacts that are injecting or non-injecting with respect to a given carrier type. One of the possible implications of the more general definition of an ohmic contact is that the free carrier density near the contact is much greater than in the bulk. Hence, the contact may act as a free carrier reservoir with the possibility of carrier injection at high fields. In applications of semiconductors as radiation detectors, this type of contact structure will act as an injecting contact.

The general definition can also be satisfied in quite a different manner. If the contact possesses a very large density of surface states that can act as recombination sites, a large recombination velocity for carriers can be achieved at the metal/diamond interface, and the contact will exhibit a very low contact resistance. The I-V characteristic in this case can also be linear. This type of ohmic contact is considered to 
be non-injecting, and contacts of this type were frequently used in fabrication of early electronic devices [48].

It is generally believed that metals that tend to form carbides make good ohmic contacts with diamond. For example, transition metals such as titanium and tantalum have been used successfully. An example I-V characteristic of a device made with $\mathrm{Ti}$ as the contact is shown in Fig. 2.2.2. It is also believed that a contact of sufficiently large area will act as an ohmic contact[49], because sufficiently large areas of contact will contain enough structural or other defects to make the contact appear ohmic. This idea essentially embraces the idea of a large surface recombination velocity but the uniformity of such contacts is uncertain. Because of its simplicity and availability, silver paint was popular in early work and is still used by many investigators [50-55]. The above reasoning may explain the success of such contacts.

\subsubsection{Schottky contacts}

A contact is considered to be Schottky in character if free movement of carriers is permitted for one bias polarity but not with the opposite polarity. This results in asymmetric I-V characteristics. A Schottky contact to diamond can be made with most metals as long as the contact is a point contact [51] or by adsorption of oxygen at the surface [52]. This result is attributed to the pinning of the Fermi level due to the surface states introduced.

In addition, the formation of a Schottky contact seems to depend on minimal solid-state reactions at the metal/diamond interface. It has been shown [52] that less reactive metals, such as $\mathrm{W}$ or $\mathrm{Au}$, form such rectifying contacts. Both $\mathrm{Al}$ and $\mathrm{Au}$ are now the metals of choice to produce rectifying contacts on all types of diamond. In Fig. 1.5.1-A is a schematic of a test structure used to measure the I-V characteristic of the $\mathrm{Au}$ contact shown in Fig. 1.5.1-B. 
A)

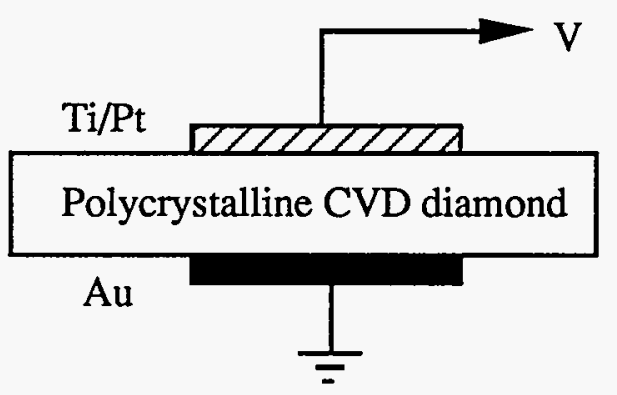

B)

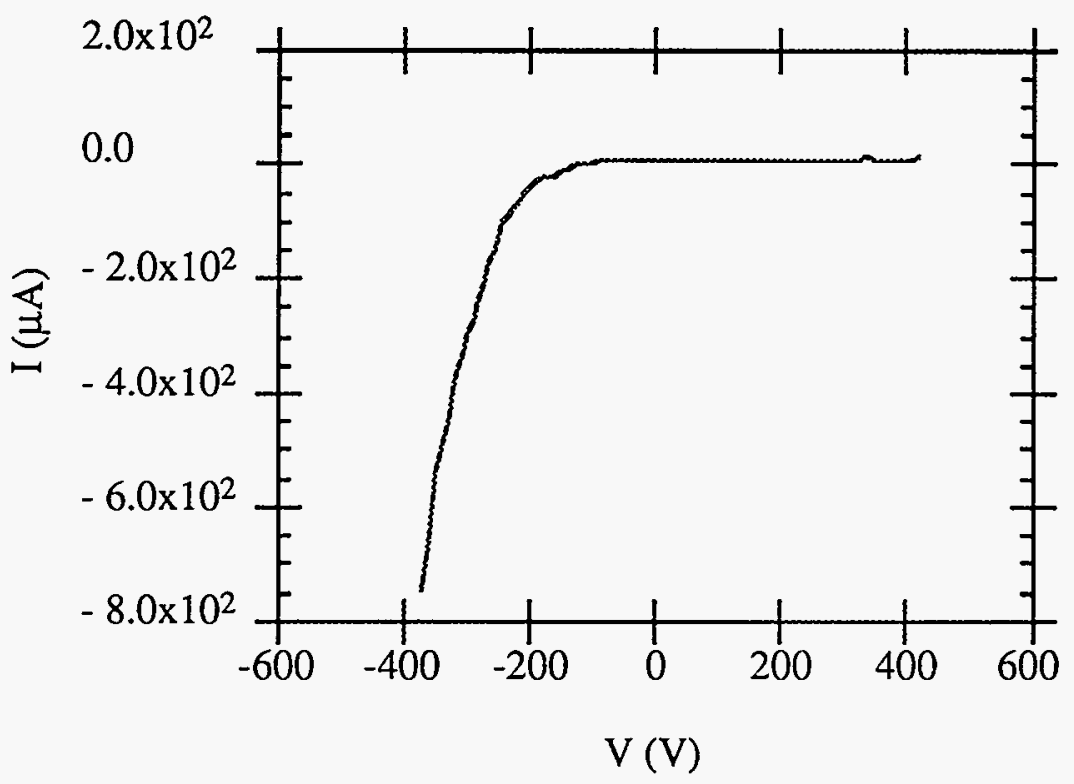

Figure 1.5.1: A) Contact geometry used to measure the Schottky diode behavior of a $50 \mu \mathrm{m}$ thick polycrystalline CVD diamond with $\mathrm{Au}$ as one contact and $\mathrm{Ti} / \mathrm{Pt}$ as the other contact.

B) I-V characteristics of the diode shown in A). 


\section{References}

[1] V. B. Gudden and R. Pohl, Z. Physik, 17 (1923) 170

[2] K. G. McKay, Phys. Rev., 77 (1950) 816

[3] R. Hofstadter, Nucleonics, 4 (1949), 2 (April) and 29 (May)

[4] A. G. Chynoweth, Phys. Rev, 83(2) (1951) 254

[5] G. P. Greeman and H. A. van der Velden, Physica, 17(6) (1951) 565

[6] F. C. Champion and S. B. Wright, Proc. Phys. Soc., 23(3) (1958) 385

[7] P. J. Kennedy, Proc. Royal Soc. London A Mat., 253 (1959) 37

[8] P. J. Dean and J. C. Male, J. Phys. Chem. Solids, 25 (1964) 311

[9] E. A. Konorova and S. F. Kozlov, Sov. Phys.-Semi., 4(10) (1972) 1600

[10] Moazed, R. Nguyen and J. R. Zeidler, IEEE Elec. Dev. Lett., 9(7) (1988) 350

[11] J. C. Angus and C. C. Hayman, Science, 241 (1988) 913

[12] L. S. Pan, S. Han, D. R. Kania, M. A. Plano and M. I. Landstrass, Diamond and Rel. Matl., in press.

[13] J. E Field, The Properties of Diamond, Academic Press, London, 1979

[14] See for example: G. Friedlander, J. W. Kennedy, E. S. Macias and J. M. Miller, Nuclear and Radiochemistry, 3rd Ed., John Wiley and Sons, New York, 1981; H. Enge, Introduction to Nuclear Physics, Addison-Wesley Publishing Co., Reading, Mass., 1966;

[15] V. S. Vavilov, Effects of Radiation on Semiconductors, Authorized Translation from the Russian by A. Tybulewicz, Consultants Bureau (1965)

[16] V. A. J. van Lint, T. M. Flanagan, R. E. Leadon, J. A. Naber and V. C. Rogers, Mechanisms of Radiation Effects in Electronic Materials, Vol. 1, John Wiley \& Sons

[17] R. H. Bube, Trans. Met. Soc. AIME, 239 (1967) 291

[18] M. DiDomenico and O. Svelto, Proc. IEEE, Feb. (1964) 136

[19] H. S. Somers and W. B. Teutsch, Proc IEEE, Feb. (1964) 144 
[20] V. T. Quat and M. A. Nicolet, IEEE Trans. Nucl.. Sci., NS-16(6) 1969

[21] V. A. van Lint and R. E. Leadon, IEEE Trans. Nuc. Sci., NS-19( 6) 1972

[22] D. K. Nichols and V. A.. van Lint, IEEE Trans. Nuc. Sci., NS-13(6),

[23] G. K. Wertheim, J. App. Phys., 30(8) (1959) 1166

[24] J. J. Loferski and P. Rappaport, J. App. Phys., 30(8) (1959) 1181

[25] B. R. Gossick, J. App. Phys., 30(8) (1959) 1214

[26] J. J. Loferski and P. Rappaport, J. App. Phys., 30(8) (1959) 1296

[27] L. W. Aukermann, J. App. Phys., 30(8) (1959) 1239

[28] E. C. Smith, D. Binder, P. A. Compton and R. I. Wilbur, IEEE Trans. Nuc. Sci., NS-13 (1966)

[29] H. Y. Fan and A. K. Ramdas, J. App. Phys., Aug. (1959) 1127

[30] G. Davies, Diamond Review, (1977) 15

[31] T. Yamaguchi, Japan J. Phys. Soc., 17(9) (1962) 1359

[32] C. D. Clark, E. W. J. Mitchell and B. J. Parsons, in the Properties of Diamond, Edited by J. E. Field, Academic Press (1979) 44

[33] J. Koike, D. M. Parkin and T. E. Mitchell, Appl. Phys. Lett., 60(12) (1992) 1450

[34] J. F. Prins, personal communication

[35] S. Han, S. G. Prussin, J. W. Ager III, L. S. Pan, D. R. Kania, S. M. Lane and R. S. Wagner, NIM, in press.

[36] M. Adel, R. Kalish and V. Richter, J. Mater. Res., 1(3) (1986) 503

[37] J. F. Prins, personal communication; D. L. Dreifus, personal communication

[38] N. R. Parikh, J. D. Hunn, E. McGucken, M. L. Swanson, C. W. White, R. A. Rudder, D. P. Malta, J. B. Posthill and R. J. Markunas, Appl. Phys. Lett., 61(26) (1992) 3124

[39] B. M. Vul, Fiz. Tverd.Tela., 3 (1961) 2264; this is a reference found in Effects of Radiation on Semiconductors by V. S. Vavilov, Trans. from the Russian, 1965 
[40] R. H. Bube, Photoconductivity of Solids, John Wiley \& Sons, 1960

[41] J. S. Blakemore, Semiconductor Statistics, Dover Publications, New York, 1982

[42] R. Dalvin, Introduction to Applied Solid State Physics, Second Ed., Plenum Press, New York, 1990

[43] J. I. Pankove, Optical Processes in Semiconductors, Dover Publications Inc., 1971; R. B. Adler, A. C. Smith, and R. L. Longini, Introduction to Semiconductor Physics, Semiconductor Electronics Education Committee, Vol. 1, Educational Services, Inc. (1964)

[44] P. J. Dean, Phys. Rev. A, 139 (1965) 588

[45] G. W. Meyer, "Search for semiconductor materials for gamma-ray spectroscopy", in Semiconductor Detectors, Edited by G. Bertolini and A. Coche, John Wiley and Sons, Inc., New York, 1968

[46] See for example: W. R. Leo, Techniques for Nuclear and Particle Physics Experiments, Springer-Verlag, Berlin, 1987

[47] See for example: J. S. Blakemore, Semiconductor Statistics, Dover Publications, Inc., New York, 1962, Chap. 2

[48] See for example E. H. Rhoderick and R. H. Williams, Metal-Semiconductor Contacts, Oxford Science Publications, Second Ed., Oxford (1988) 205

[49] M. W. Geis, J. A. Gregory and B. B. Pate, IEEE Trans. Elec. Dev., 38(3) (1991) 619

[50] L. A. Vermeulen, J. F. Young, M. I. Gallant, H. M. van Driel, Solid State Comm., 38 (1981) 1223.

[51] M. D. Bell, W. J. Leivo, Phys. Rev., 111(5) (1958) 1227.

[52] S. A. Grot, S. Lee, G. Sh. Gildenblat, C. W. Hatfield, C. R. Wronski, A. R. Badzian, T. Badzian and R. Messier, J. Mater. Res., 5(11) (1990) 2497 


\section{Chapter 2: Detector processing and experimental arrangements}

Two distinct experimental systems were developed for time-resolved current, and integrated-charge measurements. One, at the Lawrence Livermore National Laboratory, was used to measure the integrated-charge response of diamond RIC detectors to 5.48 $\mathrm{MeV} \alpha$-particles. The other, at Los Alamos National Laboratory, was used to record current transients induced by single light or heavy ions. The integrated-charge experiments were performed to determine the material properties which include the carrier mobility and lifetime, and the energy required to produce an electron-hole pair in diamond. The measurement of these properties was made by acquiring pulse-height spectra as a function of applied bias voltage and bias polarity. The experimental arrangement is described in Sec. 2.2. The pulse-height spectra also yielded information on the uniformity of the diamond material studied.

The time-resolved-current measurements were performed in an attempt to understand the dynamics of the charge collection process under light- and heavy-ion bombardment. By measuring the transient response of diamond detectors, we are able to study the effects of local excitations on the charge collection process. Furthermore, the relative importance of trapping/recombination dynamics, as compared to the loss of charge carriers at the diamond/metal-contact interface, can be examined.

Due to vastly different time scales involved in the two types measurements, the detector requirements were quite different. The integrated-charge measurements are relatively slow and require a recording system with a frequency bandwidth, or simply bandwidth, of $1.5 \mathrm{MHz}$ or less. On the other hand, time-resolved measurements require high speed and therefore bandwidths of many $\mathrm{GHz}$. In this chapter, we will discuss the detector geometry, fabrication of the detectors and the experimental arrangements used in both types of measurements. 


\subsection{Detector preparation}

The detectors used were either in the form of thin parallelepipeds with a nominal surface area of $2 \times 2 \mathrm{~mm}^{2}$, or a circular disc with diameter in the range $2.5-4 \mathrm{~mm}$. For both shapes the thickness was in the range 14.5-70 $\mu \mathrm{m}$. After receiving materials from the supplier [1], they were cleaned with a standard cleaning procedure. This was necessary because the cutting of samples was typically performed with laser cutting tools which tends to leave graphite at the edges. This can be detrimental because it represents a conductive path for carriers and can limit or prevent the application of any bias voltage.

The cleaning procedure entailed boiling the as-received material in hot chromic acid, prepared as a saturated solution of chromium trioxide in sulfuric acid, for approximately 10 minutes. This procedure has been shown to eliminate any graphite or non-diamond material, such as amorphous carbon, from the surfaces of diamond $[2,3,4$, 5]. Some care must be taken during this step because chromic acid is highly toxic. Furthermore, addition of any water into the acid, even on the level of a few drops, should be avoided because it has been found that precipitates are produced which strongly bind to the surface of diamond.

After the above step, the samples were rinsed in de-ionized water and then treated with a boiling mixture of 10 parts hydrogen peroxide (30\%) to 7 parts ammonium hydroxide (concentrated) (RCA clean [6]) for approximately 5-10 minutes to remove organic residues. They were then rinsed consecutively in dilute ammonium hydroxide and dilute hydrochloric acid. The samples were then placed in a beaker of de-ionized water in an ultrasonic cleaner for approximately 5 minutes. Finally, the samples were air dried in a convection oven at $120^{\circ} \mathrm{C}$. At this point, the samples were considered ready for processing into detectors. 
2.2 Detector processing and the experimental arrangement for integrated-charge measurements

The detectors used in the integrated-charge measurements were extremely simple metal-semiconductor-metal (MSM) devices as described in the introduction. Their principal properties of the detectors studied in the integrated-charge measurements are listed in Table 2.2.1. A shadow mask was used, as shown in Fig. 2.2.1, to define the

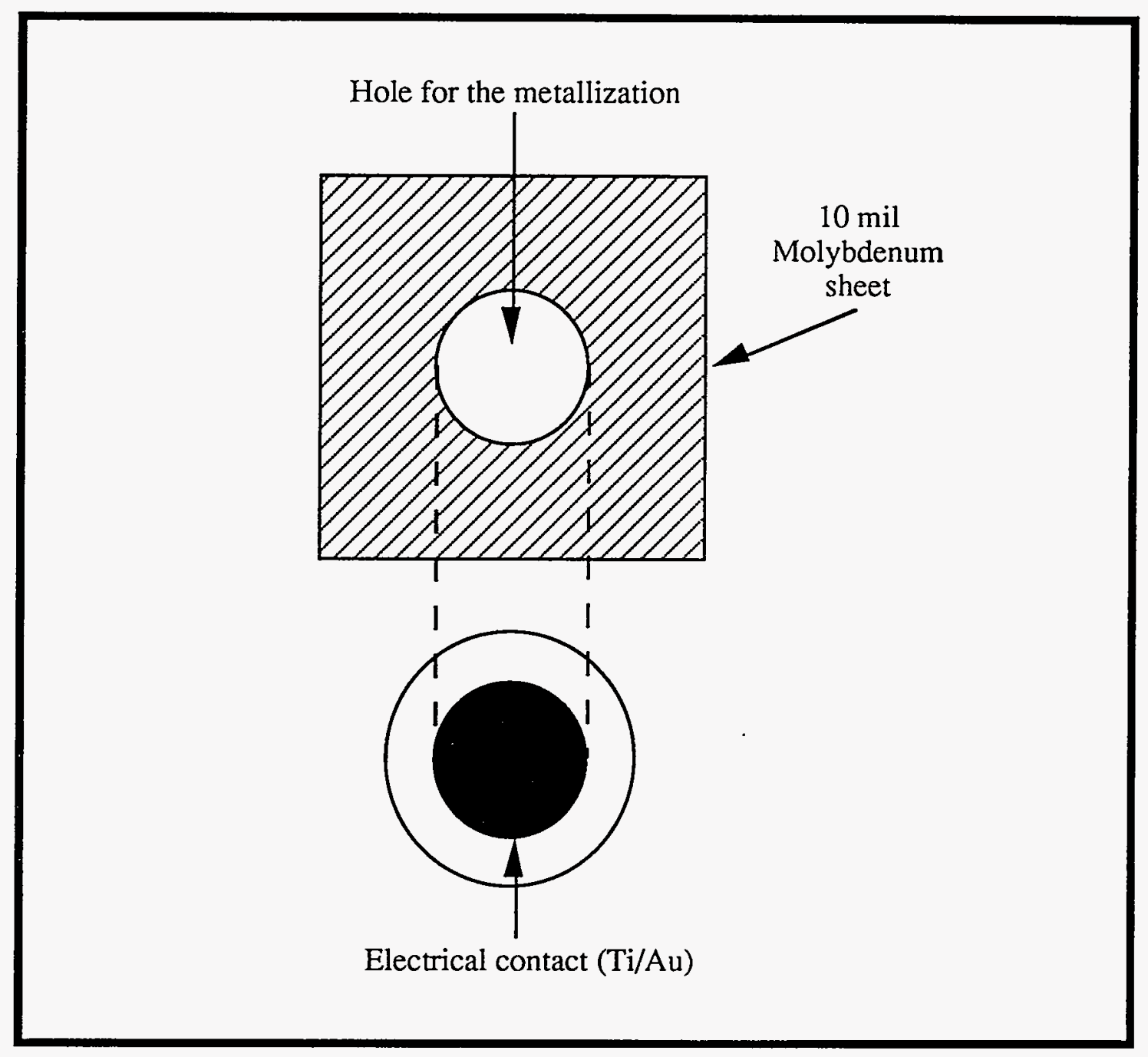

Figure 2.2.1: Shadow mask arrangement the electrical contact metalization on diamond detectors 
metallic electrical contact areas on both the top and bottom of the diamond. The shadow mask was made by cutting a $2 \mathrm{~mm}$ hole in a 10 mil thick molybdenum foil.

The sample was metalized by thermal evaporation of approximately $200 \AA$ of $\mathrm{Ti}$ followed by $5000 \AA$ of either $\mathrm{Au}$ or $\mathrm{Pt}$, or by sputtering of the contact metals onto the sample. Au or Pt was used to prevent oxidation of the Ti. The detector was annealed at $550^{\circ} \mathrm{C}$ for approximately $15-20$ minutes in air following the deposition of both metals.

The annealing step served to promote formation of low-resistivity titanium carbide at the diamond/Ti interface. It also relieved the erratic behavior of the adhesion of $\mathrm{Ti}$ to diamond when the metal was applied by thermal evaporation. This process has been shown to result in $\mathrm{I}-\mathrm{V}$ characteristics that are linear [7]. Indeed, the detectors processed in this fashion exhibited linear I-V characteristics as shown for detector SYM14m in Fig.

\subsection{2}

When sputtering was used to produce contacts, annealing was not necessary to achieve linear I-V characteristics (Fig. 2.2.3) and no difficulties with adhesion were

Table 2.2.1: Properties of detectors studied in integrated-charge experiment. Ratio is the ratio between the calculated $\alpha$-particle range (R) in diamond to the thickness $(\Delta \mathrm{Z})$ of the detector.

\begin{tabular}{|ccccc|}
\hline Detector & Method of & Detector & Detector & Ratio, \\
designation & metalization & thickness, & diameter, & $\Delta Z / R$ \\
& & $\Delta Z(\mu \mathrm{m})$ & $\mathrm{D}(\mathrm{mm})$ & \\
\hline SYM14m & Evaporation & 14.5 & 2.5 & 0.98 \\
SYM25m & Sputtering & 25 & 2.5 & 0.57 \\
SYM70m & Evaporation & 70 & 4 & 0.20 \\
\hline
\end{tabular}




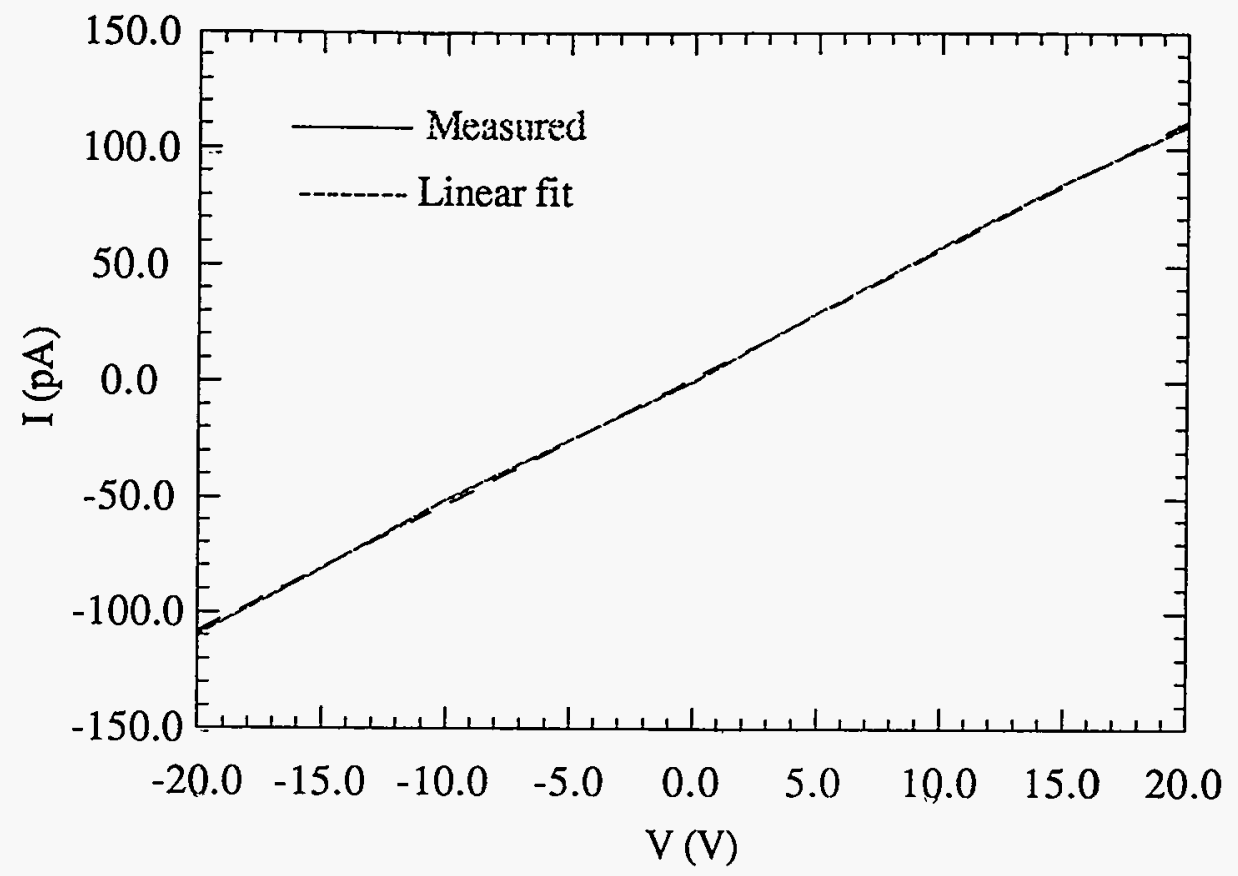

Linear fit: $I=a+b * V ; a=1.8 \pm 0.53 p A ; b=5.5 \pm 0.04\left(10^{-12} \Omega^{-1}\right)$

Quality of the fit: $R \approx 1.0$

Figure 2.2.2: I-V characteristic of thermally evaporated Ti/Au (200A Ti with $5000 \AA$ Au overlayer) contạcts on SYM14m. Correlation coefficient, $R$, equal to unity is considered to be the perfect fit. 


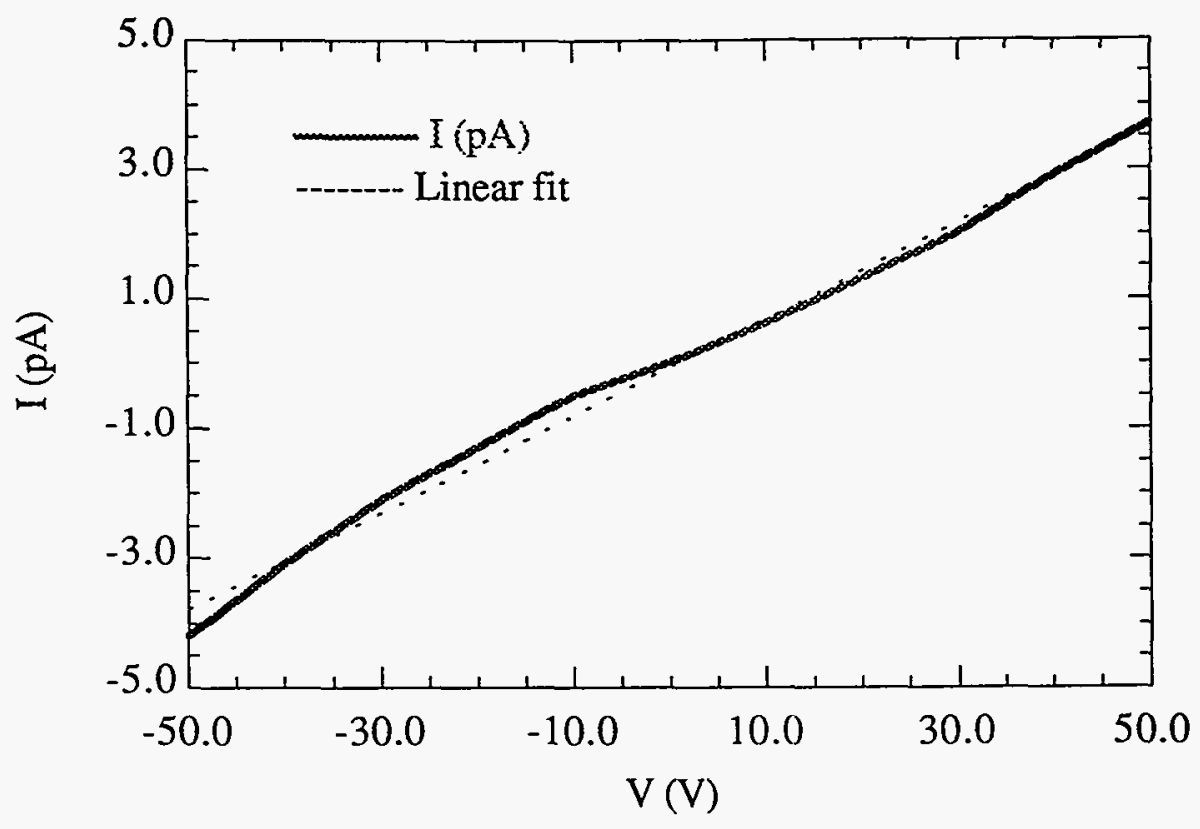

Linear fit: $\mathrm{I}=\mathrm{a}+\mathrm{b} * \mathrm{~V} ; \mathrm{a}=-0.6 \pm 0.06 \mathrm{pA} ; \mathrm{b}=0.07 \pm 0.002\left(10^{-12} \Omega^{-1}\right)$

Quality of the fit: residual $\approx 0.9$

Figure 2.2.3: $\quad \mathrm{I}-\mathrm{V}$ characteristic of sputtered $\mathrm{Ti} / \mathrm{Pt}(200 \AA \AA \mathrm{Ti}$ with $5000 \AA \mathrm{Pt}$ overlayer) contacts on SYM $25 \mathrm{~m}$.

found. It has been suggested [8] that even for kinetic energies as little as $\approx 10 \mathrm{eV}$, sputtered target atoms can implant into the substrate surface to form adherent deposits. Therefore, implantation of the sputtered Ti atoms into diamond may be the basis for better adhesion. Furthermore, it is known that $\mathrm{TiC}$ can be produced at a temperature of about $2200^{\circ} \mathrm{C}[9,10,11]$. This corresponds to a mean thermal energy of about $0.2 \mathrm{eV}$. By using TRIM [12], the mean energy of Ti atoms sputtered from a target was calculated to be $\approx 75 \mathrm{eV}$ when Ti metal is bombarded with $2 \mathrm{keV} \mathrm{Ar}^{+}$ions. Even with $\mathrm{Ar}^{+}$ions of 1 $\mathrm{keV}$, the Ti atoms sputter with $\approx 50 \mathrm{eV}$ of mean kinetic energy. Thus, direct formation of TiC appears to be possible. 
After the detectors were prepared, they were mounted in a sample holder such as the one shown in Fig. 2.2.4. The holder was designed to enable irradiation of a detector through one of the contacts. With this geometry, the particle track is parallel to applied electric field. The top connector (SMA) had a bellows attached to the end of its center pin which made electrical contact with one side of the diamond detector. Contact to the other side was made with a brass annulus of about $2 \mathrm{~mm}$ in diameter. With this arrangement, we were able to irradiate the detectors with $\alpha$-particles through the annulus which also served as a collimator. The annulus was connected to the central pin of a SHV connector whose outer conductor had a common ground with the SMA connector. As a result, bias voltage could be supplied in two different ways. In the first, the bias was applied through the SHV connector and the signal was extracted through the SMA connector. In the second, bias voltage and signals were both carried through the SMA connector. In this case, the SHV connector was terminated.

The remainder of the experimental apparatus involved in the integrated-charge measurements was comprised of standard pulse-counting electronics. A schematic of the complete arrangement is shown in figure 2.2.5.

In these experiments, $\alpha$-particles $(\approx 5.48 \mathrm{MeV})$ from a small ${ }^{241} \mathrm{Am}$ source produce free charge carriers in diamond. The average stopping time for an ion is approximated by [13]

$$
T \approx 0.12 R \sqrt{\frac{m_{A}}{E}}
$$

where $\mathrm{T}=$ average stopping time, $\mathrm{ps}$

$$
\begin{aligned}
& \mathrm{R}=\text { mean ion range, } \mu \mathrm{m} \\
& m_{A}=\text { mass of the ion, amu } \\
& \mathrm{E}=\text { initial ion energy, } \mathrm{MeV}
\end{aligned}
$$


A)
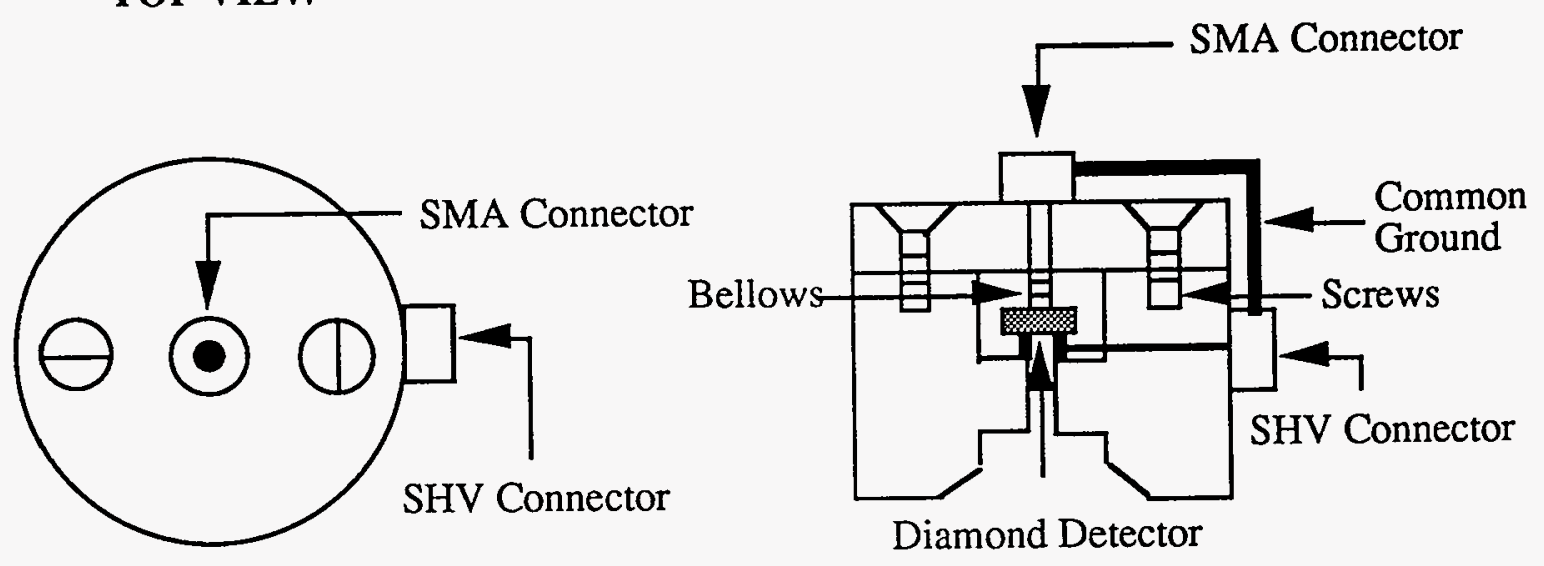

\section{REAR VIEW}

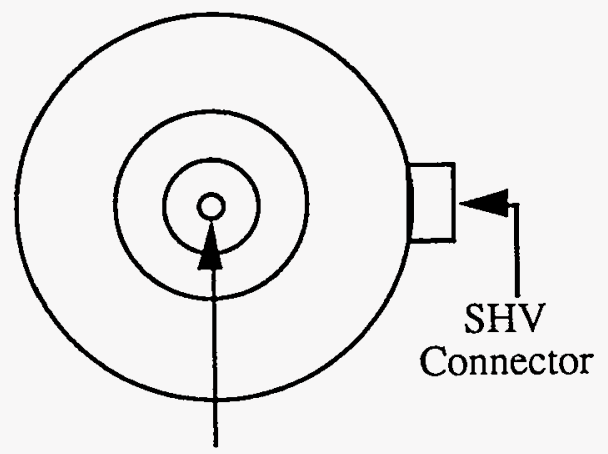

Collimating Hole

B)

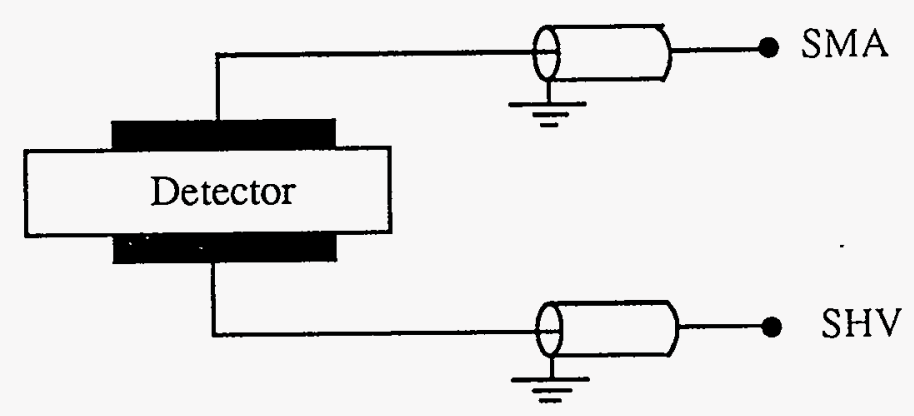

Figure 2.2.4: Schematic of the sample holder used in integrated-charge experiments: A) mechanical arrangement, B) electrical arrangement. 


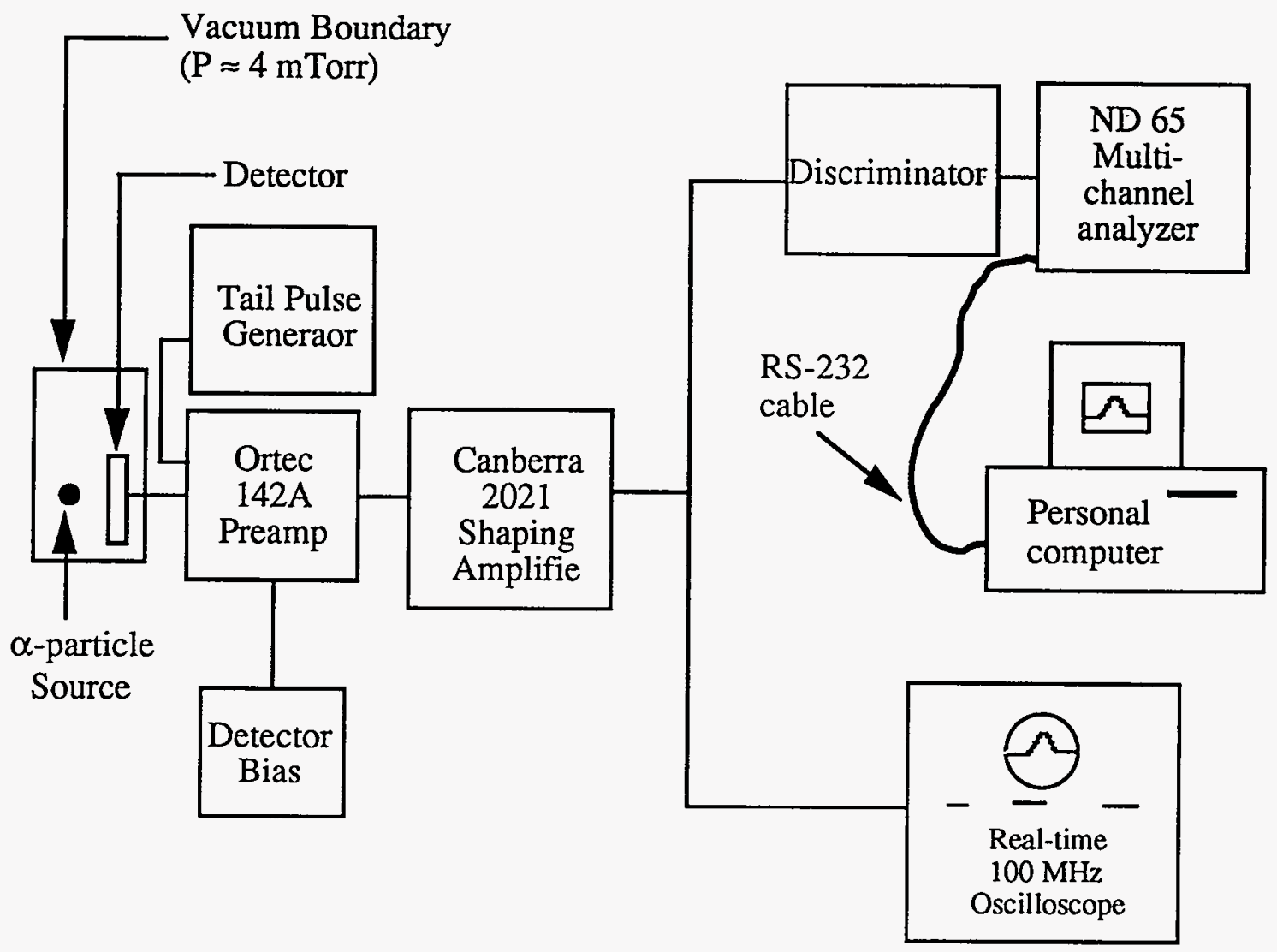

Figure 2.2.5: A schematic of the integrated-charge experimental arrangement

For these $\alpha$-particles, the average stopping time, $\tau_{\text {stop }}$, in diamond is calculated to be $\approx 1.5 \mathrm{ps}$. Under the influence of the electric field provided by an external bias voltage, carriers drift and this motion induces charge on the input capacitor of the charge-sensitive preamplifier which has a nominal clipping time, $\tau_{\text {clip }}$, of $50 \mu \mathrm{sec}$. Because the carrier lifetime, $\tau_{c}$, in diamond is about $0.1-1.0 \mathrm{~ns}$ in most of the samples studied and the RC times of the detectors used are less than $\approx 1 \mathrm{~ns}, \tau_{\text {clip }}$ is essentially infinite in comparison. In summary $\tau_{\mathrm{clip}} \gg \tau_{\mathbb{\square}}, \tau_{\mathrm{RC}}, \tau_{\mathrm{c}}, \tau_{\text {stop }}$ (Table 2.2.2), and this ensures that the preamplifier output signal will be accurately proportional to the collected charge. We note that even if the carrier lifetime approached the range of tens of nanoseconds, the transit time, $\tau_{t r}$, for 
Table 2.2.2: List of relevant characteristic times in integrated-charge measurement.

\begin{tabular}{|ccccc|}
\hline$\tau_{\text {stop }}(\mathrm{ns})$ & $\tau_{\mathrm{c}}(\mathrm{ns})$ & $\tau_{\mathrm{tr}}(\mathrm{ns})$ & $\tau_{\mathrm{RC}}(\mathrm{ns})$ & $\tau_{\mathrm{clip}}(\mathrm{ns})$ \\
\hline$\approx 1.5 \times 10^{-3}$ & $<0.1-1.0$ & $\geqq 0.1$ & $\approx 0.2-1.0$ & 50000 \\
\hline
\end{tabular}

a typical detector of $20 \mu \mathrm{m}$ thickness at a saturation velocity of $2 \times 10^{7} \mathrm{~cm} / \mathrm{sec}$ is only about 100 ps.

The output of the preamplifier was applied to the input of a Gaussian shaping amplifier with variable shaping time. The shaping time was chosen to be $1 \mu$ s to maximize the signal-to-noise ratio and hence the overall resolution of the system. Electronic noise was constantly monitored from the preamplifier input through the amplifier by injecting a charge pulse from a tail-pulse generator to the preamplifier test input. The amplifier output was then routed to a multichannel analyzer where pulseheight spectra were measured and stored. Spectra were then transferred to a computer for detailed spectral analysis.

The noise from the detectors appears as voltage $\left(V_{r m s}\right)$ or current $\left(I_{r m s}\right)$ fluctuations, where subscript rms refers to root mean square. Because the signal from our detectors is measured in electric charge, a convenient way to express the different noise contributions in the detection system (see Fig. 2.2.5) is in terms of the equivalent-noise charge (ENC) in units of the electron charge. Because our detectors were capacitively coupled to the detection electronics, ENC is given by

$$
E N C=\frac{C_{T} V_{m s}}{e}
$$

where $C_{T}=$ total input capacitance which is the sum of the detector capacitance, $C_{D}$, and any other capacitance from the rest of the electronics, $C_{I}$. It is clear that any increase in the total input capacitance comes from the detector since the capacitance contribution from the electronics is fixed. Therefore, the minimization of 
the detector capacitance becomes important. For all detectors used here, the maximum detector capacitance was less than $20 \mathrm{pF}$, well within the range recommended by the preamplifier manufacturer.

A potentially large contribution to the total noise in a detection system is the detector leakage current. This current can be visualized as being formed by individual free electrons and holes crossing through or on the surface of the detector. Fluctuations in the number of these carriers give rise to noise. For diamond, this contribution to the total noise in the system should be negligible due to high inherent resistivity of the diamond. The total noise, as measured by the full-width-at-half-maximum (FWHM) of the pulser peak was typically $2069 \pm 142$ electrons. The full-energy peak widths from all detectors studied were greater than $\approx 2 \times 10^{4}$ electrons (see Sec. 3.1). Therefore, the total electronic noise does not contribute significantly to the widths of these peaks.

The main radioactivity used to determined the detector response to $\alpha$-particles was ${ }^{241} \mathrm{Am}$ as supplied by Isotope Products, Inc. The intensity of this source was less than $1 \mu \mathrm{Ci}$ and the radioisotope was electroplated onto a $\mathrm{Pt}$ foil. Due to the low activity, no protective window covered the source and hence energy dispersion in the $\alpha$-spectrum was minimal and was dominated by the actual source thickness.

${ }^{241} \mathrm{Am}$ has three main $\alpha$-groups with energies (intensities) of $5.48 \mathrm{MeV}(86 \%)$, $5.44 \mathrm{MeV}(12.7 \%)$, and $5.39 \mathrm{MeV}(1.33 \%)$. The mean energy of the $\alpha$-particles is calculated to be $5.47 \mathrm{MeV}$. Within an error of approximately $0.1 \%$, we are justified in assuming a single $\alpha$-group with the energy of $5.48 \mathrm{MeV}$. Because the maximum energy difference between the $\alpha$-groups corresponds to about 6000 electrons in diamond, and because the experimental FWHM of the full-energy peak in all measured spectra was far greater than this, negligible error is introduced by this approximation.

Another potential source of line-broadening is the energy dispersion caused by the contact metals which act as an entrance window through which the $\alpha$-particles must penetrate to reach the detector. By acquiring pulse-height spectra before and after the 
insertion of a free-standing, $5000 \AA$ thick $A u$ foil between a silicon surface barrier detector and the ${ }^{241} \mathrm{Am}$ source, we were able to measure this effect. (The effect on the energy dispersion by the additional $200 \AA$ of $T i$ is negligible because of its thinness and its low atomic number in comparison to Au.) Spectra obtained in this way are shown in Fig. 2.2.6. The FWHM of the $\alpha$-peak before and after the insertion of the foil were 34.2 $\mathrm{keV}$ and $51.4 \mathrm{keV}$, respectively. The noise contribution to the full-energy peak from the detector capacitance, leakage current, and the electronics was determined to be $17.1 \mathrm{keV}$ with use of the tail-pulse generator. Assuming that the noise contributions and the energy dispersion add in quadrature, the line broadening due to energy dispersion in the foil is about $34.2 \pm 3.4 \mathrm{keV}$. Therefore, the contribution of the energy dispersion on the fullenergy peak should be less than $1 \%$.

The mean energy loss of the $\alpha$-particles after penetrating the metal contact was determined from the centroid shift seen in Fig. 2.2 .6 to be $251.1 \pm 0.7 \mathrm{keV}$. This loss is less than $5 \%$ of the initial $\alpha$-particle energy. Therefore, by neglecting this energy loss in subsequent data analysis, we would introduce an error of less than $5 \%$.

In order to establish a reliable energy scale, a pulse-height calibration relative to a silicon surface barrier detector [14] was performed. For this purpose a source of ${ }^{228} \mathrm{Th}$ in equilibrium with its radioactive daughters was used. The energies of important $\alpha$-groups from this source are listed in Table 2.2.3. The depletion depth of the silicon detector was $52.1 \mu \mathrm{m}$ which allowed full stopping of all $\alpha$-particles. To establish a good absolute energy scale, the energy needed to produce an electron-hole pair in $\mathrm{Si}\left(\boldsymbol{\varepsilon}_{\mathrm{si}}\right)$ must be well known. In fact $\varepsilon_{\mathrm{si}}$ has been measured to be $3.61 \pm 0.02 \mathrm{eV}$ [15]. Because the error in this property is much less than $1 \%$, it will be neglected in subsequent analysis. 


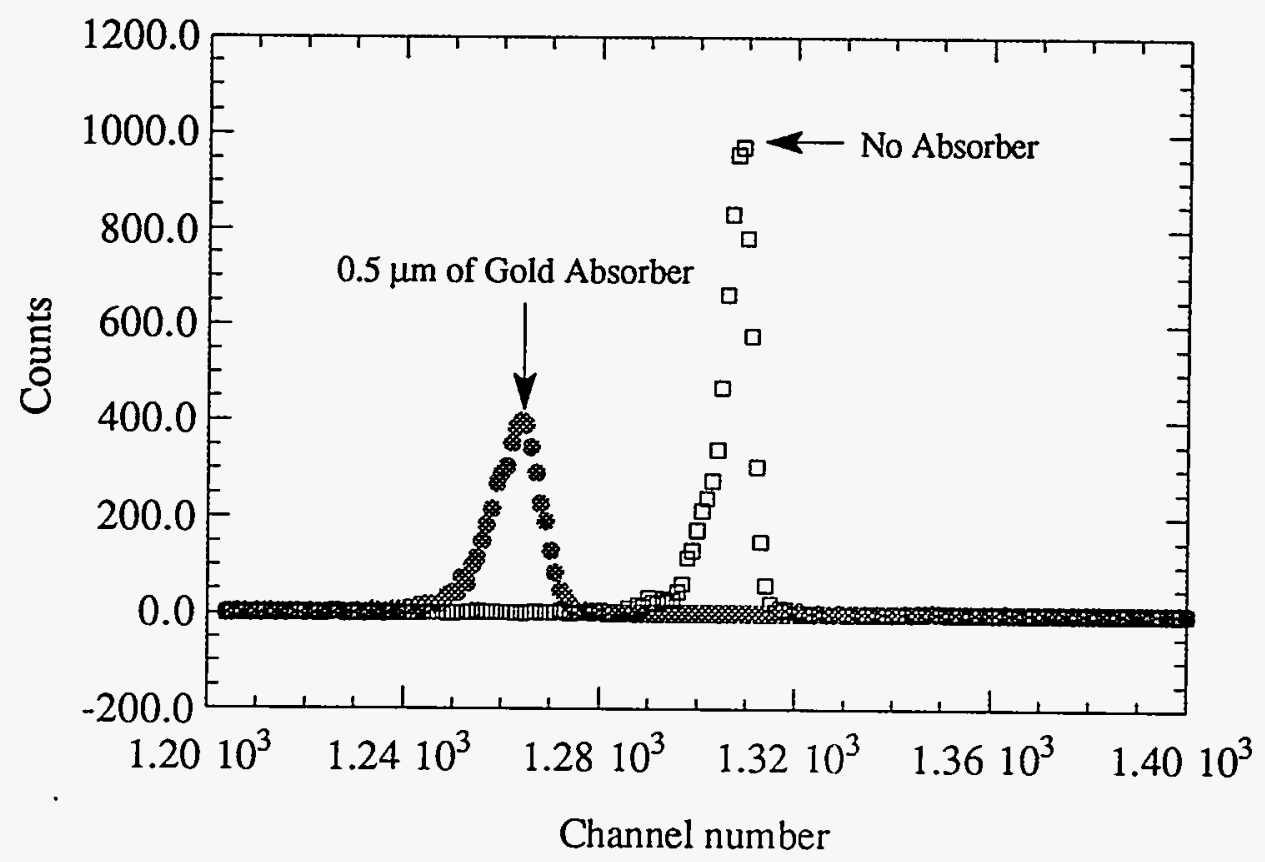

Figure 2.2.6: The effect of $5000 \AA$ thick gold foil on 5.48-MeV $\alpha$-particles from an ${ }^{241} \mathrm{Am}$ source. The energy loss is approximately $251 \mathrm{keV}$ and the full-width-at-half-maximum of the attenuated and unattenuated $\alpha$-peaks are $51.4 \mathrm{keV}$ and $34.2 \mathrm{keV}$, respectively. The noise contribution in both measurements was determined to be $17.1 \mathrm{keV}$. 
Table 2.2.3: The significant $\alpha$-groups from ${ }^{228}$ Th source. The radioisotopes other than ${ }^{228} \mathrm{Th}$ are the decaying daughters in equilibrium with ${ }^{228} \mathrm{Th}$.

\begin{tabular}{|ccc|}
\hline $\begin{array}{c}\text { Energy } \\
(\mathrm{MeV})\end{array}$ & Radioisotope & $\begin{array}{c}\alpha \text {-particle range in silicon } \\
(\mu \mathrm{m})\end{array}$ \\
\hline 5.34 & $228 \mathrm{Th}$ & 23 \\
5.42 & $228 \mathrm{Th}$ & 24 \\
5.68 & $224 \mathrm{Ra}$ & 25 \\
6.05 & $212 \mathrm{Bi}$ & 30 \\
6.09 & $212 \mathrm{Bi}$ & 30 \\
6.29 & $220 \mathrm{Rn}$ & 32 \\
6.78 & $216 \mathrm{Po}$ & 38 \\
8.78 & $212 \mathrm{Po}$ & 51 \\
\hline
\end{tabular}


2.3 Detector fabrication and experimental arrangement for the time-resolved current experiments

Unlike those described in section 2.2 , the detector construction used in the timeresolved measurements is complicated by the speed at which the devices and the measurement systems must operate. In order to examine the speed requirements for recording an electrical transient caused by an ion, we will examine the interaction of a 5$\mathrm{MeV} \alpha$-particle in diamond. For this ion, the average stopping time, $\tau_{\text {stop, }}$ is approximately $1.3 \mathrm{ps}$ using the mean range of $12 \mu \mathrm{m}$ calculated with the computer code TRIM and Equ. 2.2.1. Assuming a mean radius of $1 \mu \mathrm{m}$ for the ion track (see Sec. 3.2.2), the average free electron-hole density produced from ionization is roughly estimated to be $\approx 10^{16} \mathrm{~cm}^{-3}$ assuming $\varepsilon_{\mathrm{di}}=14.2 \mathrm{eV}$ (see Sec. 3.2.2). Due to creation of this plasma, there will be dielectric collapse in its center. (This effect will be discussed in Chap. 4 in detail.) The time scale, $\tau_{\text {diel }}$, for this type of relaxation is estimated to be on the order of a few hundred femtoseconds and the plasma time, $\tau_{\mathrm{P}}$, which is defined as the time needed to erode the plasma to a point where normal charge collection can proceed, is on the order of tens of picoseconds. In the limit of zero plasma density, the carrier lifetime, $\tau_{c}$, is expected to be approximately $0.1-1 \mathrm{~ns}$. Assuming charge carriers reach their saturation velocities of about $2 \times 10^{7} \mathrm{~cm} / \mathrm{sec}$, and assuming a detector width of $20 \mu \mathrm{m}$, the minimum carrier sweep-out time, $\tau_{\text {tr }}$, is expected to be approximately $100 \mathrm{ps}$. Therefore, if we wish to observe dielectric relaxation, plasma erosion, and wish to follow the trapping/recombination dynamics, we need a measurement system which can operate with rise times of less than a picosecond. Even the observation of the free carrier decay through trapping/recombination alone requires a recording system rise time of less than 100 ps. Consequently, the rise time of a measurement system must be as short as possible. The rise time as a function of bandwidth can be approximated by [16] 


$$
T_{r}[p s]=\frac{1}{3 \Delta f[G H z]}
$$

where $T_{r}=$ rise time

$$
\Delta f=\text { bandwidth }
$$

Thus, we can see that the measurement apparatus and the detector together must be able operate with the minimum total bandwidth of about $10 \mathrm{GHz}$. Table 2.3 .1 summarizes the relevant time scales in these experiments.

Table 2.3.1: List of relevant time scales for time-resolved-current experiments

\begin{tabular}{|ccccc|}
\hline$\tau_{\text {stop }}(\mathrm{ps})$ & $\tau_{\text {diel }},(\mathrm{ps})$ & $\tau_{\mathrm{P}}$ & $\tau_{\mathrm{c}}(\mathrm{ps})$ & $\tau_{\mathrm{tr}}(\mathrm{ps})$ \\
\hline$\approx 1.3$ & $\ll 1$ & $\approx$ tens of & $\approx 100-1000$ & $\approx 100$ \\
& & picoseconds & & \\
\hline
\end{tabular}

\subsubsection{Detector fabrication}

The diamond detectors used in these experiments were incorporated into doubleended microstrip transmission lines, referred to informally as microstrips, similar to the high-speed photoconductors used as optoelectronic devices by Lee [17] and Auston[18]. A schematic and a photograph of the complete detector-microstrip structure are shown in Fig. 2.3.1. The applied electric field distribution in these detectors is nearly uniform [19] and is collinear with the microstrips. A microstrip can be described as the planar analog of a coaxial cable. It is formed from a strip of conductor located on top of a dielectric that has a conductive backing that serves as ground plane (Fig. 2.3.2-A). In comparison, a coaxial cable has a conductor surrounded by a dielectric which is itself surrounded by an outer conductor (Fig. 2.3.2-B). Similar to a coaxial cable that has a characteristic impedance that is a function of the radii of the inner and the outer conductors, a microstip 
A)

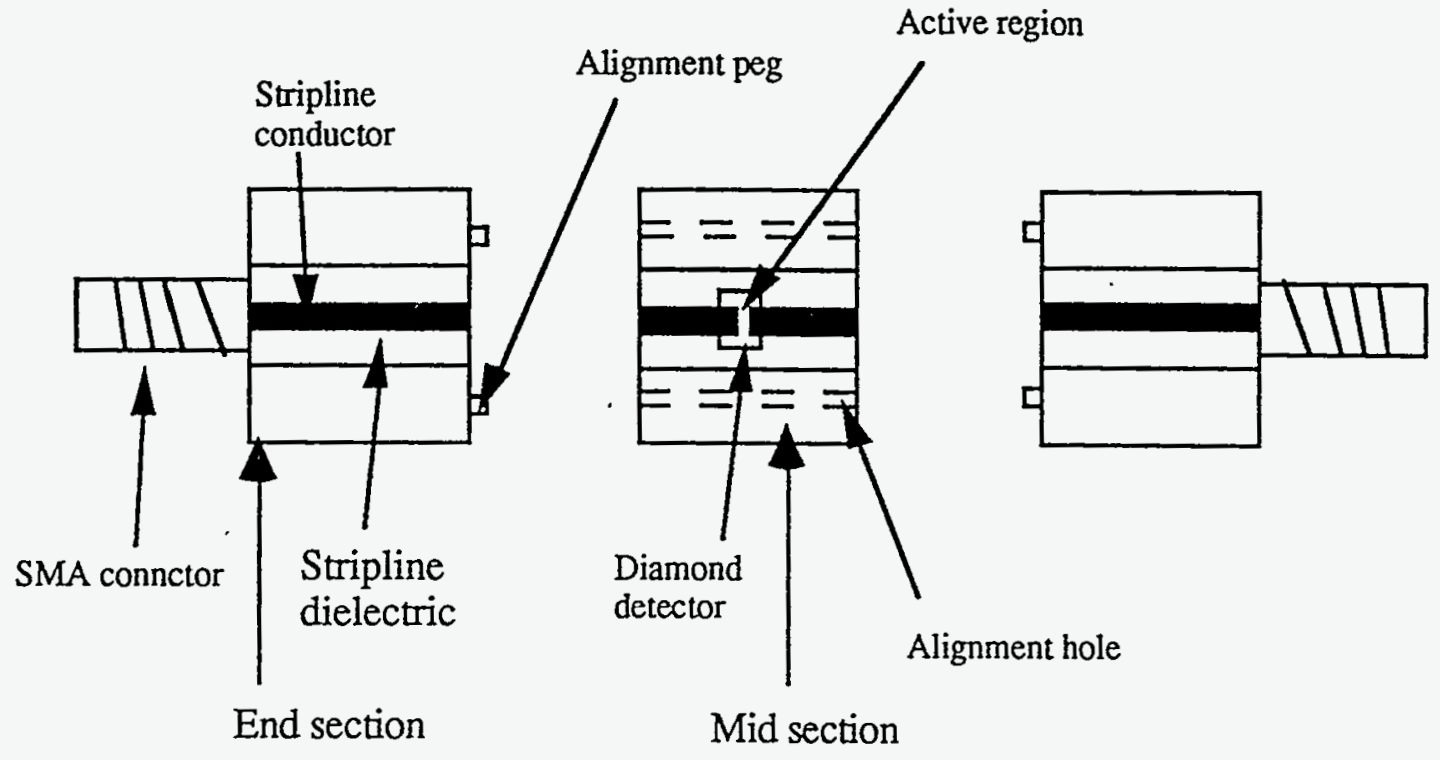

B)

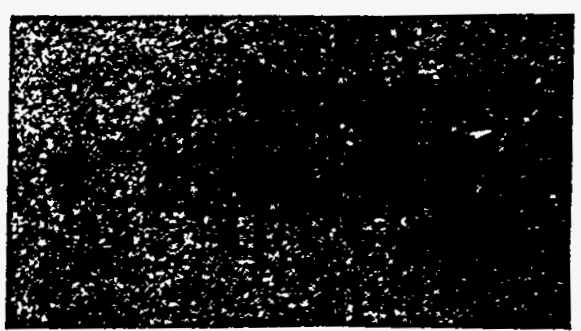

C)

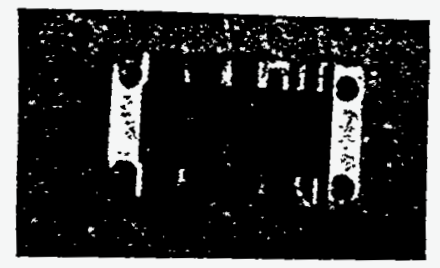

Figure 2.3.1: Schematic and photograph of complete detector packages used for the time-resolved current experiments; A) schematic of the detector mounted on a Hewlett Packard (HP) microwave circuit package shown in B); B) photograph of a Hewlett Packard (HP) microwave circuit package; C) photograph of a microstrip with monolithic dielectric substrate. The overall speed of package shown in (C) is higher than the package shown in (C) mainly due to the differences in the bandwidth of the SMA connectors. 
A)

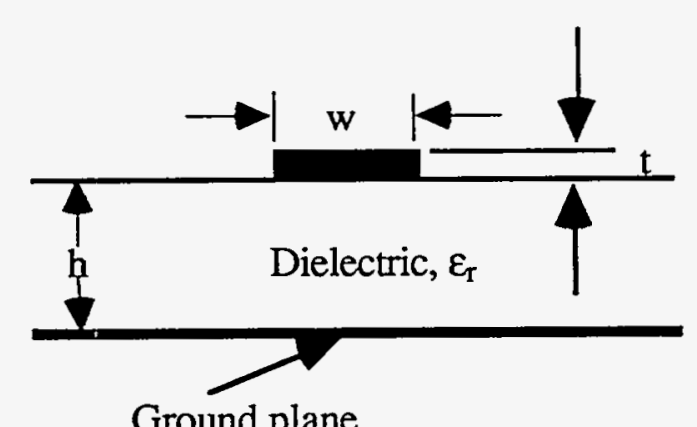

$=$ conductor
B)

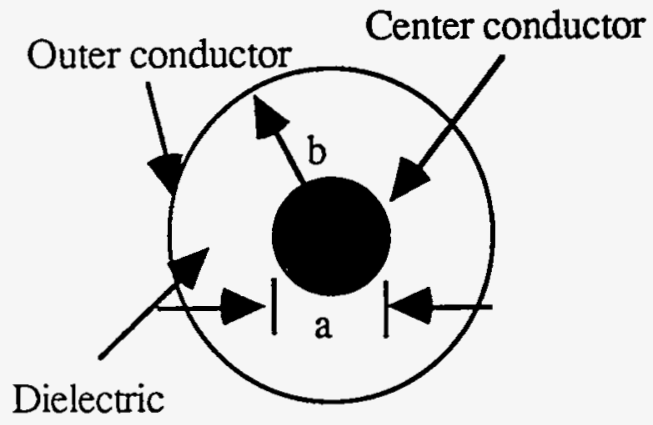

$\mathrm{a}=$ diameter of the center conductor

$\mathrm{b}=$ radius of the outer conductor

Figure 2.3.2: Schematics of two different transmission lines. A) A microstrip transmission line. B) A coaxial cable with a central conductor buried between a dielectric, with the thickness of $(b-a / 2)$, and an outer conductor.

has a characteristic impedance, $Z_{0}$, that is a function of the width, $w$, and the thickness, $t$, of the upper conductor, and the thickness, $h$, and relative permittivity, $\varepsilon_{\mathrm{r}}$, of the dielectric. The characteristic impedance of a microstrip can be expressed as [20]

$$
Z_{o}=\left\{\begin{array}{l}
\frac{120 \pi}{2 \pi \sqrt{\varepsilon_{e f f}}} \ln \left(\frac{8 h}{W_{e}}+0.25 \frac{W_{e}}{h}\right) ;(w / h \leq 1) \\
\frac{120 \pi}{\sqrt{\varepsilon_{\text {eff }}}} \ln \left[\frac{W_{e}}{h}+1.393+0.667 \ln \left(\frac{W_{e}}{h}+1.444\right)\right]^{-1} ;(w / h \geq 1)
\end{array}\right.
$$

where $t=$ upper conductor thickness

$$
\begin{aligned}
& \varepsilon_{\text {eff }}=\text { effective relative permitivity } \\
& \quad=\frac{\varepsilon_{r}+1}{2}+\frac{\varepsilon_{r}-1}{2} F(w / h)-C \\
& F(w / h)=\left\{\begin{array}{l}
\left(1+12(h / w)^{-1 / 2}+0.04(1-w / h)^{2} ;(w / h \leq 1)\right. \\
\left(1+12(h / w)^{-1 / 2} ;(w / h \geq 1)\right.
\end{array}\right.
\end{aligned}
$$




$$
\begin{aligned}
& C=\frac{\varepsilon_{r}-1}{4.6} \frac{t / h}{\sqrt{w / h}} \\
& \frac{W_{e}}{h}=\left\{\begin{array}{l}
\frac{w}{h}+\frac{1.25}{\pi} \frac{t}{h}\left[1+\ln \left(\frac{4 \pi}{t}\right)\right] ;(w / h \leq 1 / 2 \pi) \\
\frac{w}{h}+\frac{1.25}{\pi} \frac{t}{h}\left[1+\ln \left(\frac{2 h}{t}\right)\right] ;(w / h \geq 1 / 2 \pi)
\end{array}\right.
\end{aligned}
$$

For the construction of the microstrips used in this work, the dielectric was alumina $\left(\varepsilon_{\mathrm{r}} \approx 9.5\right)$. Because the input impedance of our recording system (see Sec. 2.3.2) was $50 \Omega$, the characteristic impedance of the microstrips had to be of the same magnitude. With the known dielectric and $\mathrm{Z}_{0}$, equations $2.3 .2-2.3 .6$ were used to determine the ratio, $w / h$, and it was calculated to be near unity.

As stated previously, the rise time of the microstrip must be as small as possible, which implies that the bandwidth of the structure should be at least as large as the recording system bandwidth. The bandwidth of the microstrip can be estimated by [21]

$$
f_{T E}=\frac{c}{4 h \sqrt{\varepsilon_{r}-1}}
$$

where $f_{T E}=$ transition frequency from transverse electromagnetic (TEM) mode of electromagnetic wave propagation to other higher modes such as transverse electric (TE), $\mathrm{Hz}$ $c=$ speed of light in vacuum

The transition to higher modes of wave propagation in microstrips will lead to distortions of the pulse being propagated [21]. Therefore, to ensure the fidelity of the pulse propagating from a current transient, the bandwidth of the detector-microstrip structure is essentially $f_{T E}$. By inspecting equations 2.3 .1 and 2.3.7, it is clear that the rise time of the detector package is ultimately a function of the thickness of the dielectric; the thinner the dielectric, the higher the bandwidth and the narrower must be the upper conductor. If 
we assume a required rise time of $100 \mathrm{fs}$, the thickness of the alumina must be about 8 $\mu \mathrm{m}$ if we hope to observe dielectric relaxation in the detector even if the rise time of a recording system is zero. For a 1-ps rise time, the alumina thickness is larger by an order of magnitude. We can thus see that just to achieve a 1-ps rise time, the physical dimensions of the dielectric are very small. Since alumina tends to be very brittle, handling becomes a serious issue.

It is important that the resistance of the upper conductor must be negligible in comparison to the impedance of the detector-microstrip package because any conductor resistance appears as an additional series resistance when calculating the total impedance included in the RC time constant of the detector (see below) at DC or near DC operation. At high signal frequencies, the conductor resistance differs from the DC value. The current density distributions in the conductors of the microstrip are not uniform throughout the thickness of the conductors. Instead, the current density distributions are concentrated within narrow regions close to the conductor surfaces. The distance over which the current density attenuates by a factor of $1 / \mathrm{e}$ is called the skin depth. The skin depth is a function of the signal frequency (f) and the conductor resistivity $(\rho)$, and is given by

$$
\delta=\sqrt{\frac{\rho}{\pi f \mu}}
$$

where $\mu=$ permeability

The dependence of $\delta$ on frequency is shown in Fig. 2.3.3. Therefore, the equivalent resistance is calculated based on the skin depth as

$$
R_{s}=\frac{\rho}{\delta}
$$

The resistance of the conductor at high frequencies is not dictated by the cross sectional area of the conductor but rather it is controlled by the perimeter of the cross section. So, 


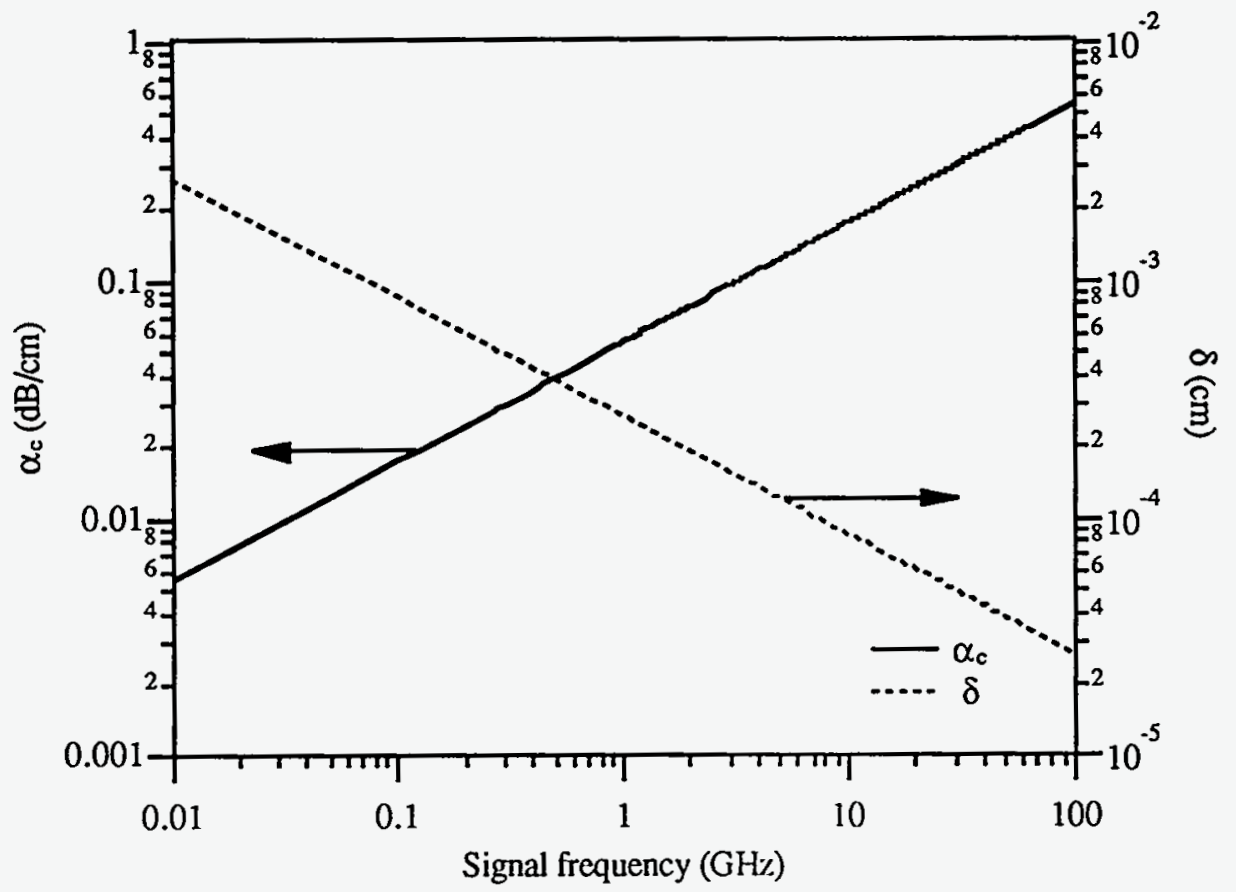

Figure 2.3.3: Gold-conductor loss $\left(\alpha_{c}\right)$ and skin depth $(\delta)$ as a function of propagating signal frequency with alumina as the dielectric substrate and with the ratio $w / h=1$.

depending on the operating frequency range, the thickness of the conductors should be about $4.6 * \delta$ to encompass greater than 0.99 of the current density distribution in the conductors. Of course, when operating near DC conditions, the resistance of the conductors is based on the cross sectional area. Furthermore, if the upper conductor is thinner than $\delta$, then conductor losses can occur that will cause loss in the fidelity of the propagating pulse which can lead to degradation of the pulse rise time. The conductor loss as a function of the conductor thickness [22] is approximated by

$$
\alpha_{c}=\left\{\begin{array}{l}
1.38 A\left[\frac{R_{s}}{h Z_{o}}\right]\left[\frac{32-\left(W_{e} h\right)^{2}}{32+\left(W_{e} / h\right)^{2}}\right] ;(w / h \leq 1) ; \mathrm{dB} / \text { unit length } \\
6.1 \times 10^{-5} A\left[\frac{R_{s} Z_{o} \varepsilon_{e f f}}{h}\right]\left[\left(W_{e} / h\right)+\frac{0.667\left(W_{e} / h\right)^{2}}{1.444+\left(W_{e} / h\right)}\right] ;(W / h \geq 1) ; \mathrm{dB} / \text { unit length }
\end{array}\right.
$$


where $A=1+\left(\frac{h}{W_{e}}\right)\left\{1+\left(\frac{1.25}{\pi}\right) \ln \left(\frac{2 B}{t}\right)\right\}$

$$
B= \begin{cases}h \quad & (w / h \geq 1 / 2 \pi) \\ 2 \pi w & ;(w / h \leq 1 / 2 \pi)\end{cases}
$$

The conductor loss as a function of frequency is shown in Fig. 2.3.3.

Based on such considerations, a thickness of $250 \mu \mathrm{m}$ was chosen for the dielectric (corresponding to a upper-conductor width of $250 \mu \mathrm{m}$ ). For this thickness, the bandwidth of a microstrip was estimated to be $103 \mathrm{GHz}$ (Eq. 2.3.3) and its rise time was estimated to be 3.2 ps. While this is much greater than the dielectric relaxation time, it is below the anticipated plasma time.

The microstrip used was either a modular microwave circuit package acquired from Hewlett Packard (HP) (Fig. 2.3.1-B) or a monolithic type (Fig. 2.3.1-C), where the diamond sample was mounted at its center (Fig. 2.3.1A). The upper conductor was either $\mathrm{Au}$ or $\mathrm{Pt}$ with $\mathrm{W} / \mathrm{Ti}$ alloy or $\mathrm{Ti}$, respectively, as an adhesion layer between the conductor and the dielectric. The series resistance of the conductors was less than $1 \Omega$. The package used for the majority of the measurements was the HP package because its response time proved to be smaller by about $10 \%$. The active detector, defined using a standard photolithography and lift-off process (see App. A), is a $20 \mu \mathrm{m}$ wide gap on the diamond with a total area of $5 \times 10^{-5} \mathrm{~cm}^{2}$. The microstrip on diamond, which also acts as an electrode on the detector, was made by thermal evaporation of $300 \AA$ of titanium followed by $3000 \AA$ of gold. These contacts were annealed under the same conditions described previously (see Sec. 2.2). The DC resistance of the Au-conductor was calculated to be $0.3 \Omega$. Therefore, the total contribution to the RC constant from the series resistance of the conductor at near DC operation and the resistance of the conductor at high frequencies $(\approx 0.1 \Omega$ ) is negligible, and the RC time constant should be roughly determined by the $Z_{0}$ of the microstrip and the gap capacitance of the detector. The skin depth and the conductor loss were calculated to be $\approx 2600 \AA$ and $\approx 0.5 \mathrm{~dB} / \mathrm{cm}$, 
respectively, at $100 \mathrm{GHz}$. Because the thickness of the upper conductor with the HP package is about $2.5 \mu \mathrm{m}$, the largest conductor loss should appear with the conductor on the detector. The length of the conductor on the detector was approximately $1 \mathrm{~mm}$. Thus, the total conductor loss on the detector was calculated to be $\approx 0.05 \mathrm{~dB}$. Even at 10 $\mathrm{GHz}$ the conductor loss is only about $0.02 \mathrm{~dB}$. Therefore, the fidelity of the propagating pulse should not be greatly affected.

The ultimate limit to the rise time of any detector structure is the RC time constant associated with the active detector area. By graphically interpreting the results of Maeda [23], the capacitance of the active area of our detector was estimated to be about $80 \mathrm{fF}$. The RC time constant associated with the gap was then estimated to be $\approx 4 \mathrm{ps}$. This corresponds to a bandwidth of $83 \mathrm{GHz}$. Therefore, the total rise time ( $\mathrm{RC}+$ microstrip) was estimated to be about 5 ps. With this time scale, we may be able to observe the plasma time directly but any evidence for dielectric collapse must be gathered indirectly. The prospects for indirect observation will be discussed in Chap. 4 in more detail.

In the frequency range of tens of $\mathrm{GHz}$, impedance matching of all components is critical to ensure the fidelity of any electrical pulse. Impedance discontinuities at the boundaries of the gap, and impedance mismatches present in the microstrip were examined with a Tektronix CSA803 sampling oscilloscope equipped with a $25 \mathrm{GHz}$ sampling head. A 20-ps step pulse generated within the oscilloscope was introduced into the microstrip and the transmitted pulse recorded. Because the gap is essentially an RCdifferentiator, the transmitted step-pulse was approximately Gaussian in shape at the output. In order to compare the original and the transmitted pulses, the step pulse was differentiated and recorded. A comparison of the latter pulse with the transmitted pulse is seen in Fig. 2.3.3. Degradation in rise time of the original pulse appears to be minimal, with only small reflections from discontinuities and impedance mismatches. For comparison, the transmitted waveform associated with a badly constructed microstrip is 
shown in Fig. 2.3.4. A simple Gaussian waveform is nowhere to be found. Many reflections are indicated and these are indicative of significant impedance mismatches.

Before an experiment, an assessment of the response of the entire detector package to impulse excitation was accomplished through transient photoconductivity (TPC) [24] induced with 6-ps UV laser pulses. The experimental arrangement for this measurement is described in App. B. Any gross reflections resulting from impedance mismatches can be seen with this method because it utilizes a $50-\Omega$ high-speed recording system with a rise time of approximately $70 \mathrm{ps.} \mathrm{A} \mathrm{typical} \mathrm{pulse} \mathrm{taken} \mathrm{with} \mathrm{a} \mathrm{detector}$ package used in 5-MeV He${ }^{+}$ion excitation measurements is shown in Fig. 2.3.5. The response of the package seems very clean. Only one signal reflection is evident $600 \mathrm{ps}$ following the start of the laser excitation.

The speed, $v$, of the wave propagation in a dielectric can be determined by

$$
\nu=\frac{c}{\sqrt{\varepsilon_{r}}}
$$

For alumina, $v \approx 1 \times 10^{10} \mathrm{~cm} / \mathrm{sec}$. The time interval of $600 \mathrm{ps}$ is equivalent to a propagation dimension of about $6 \mathrm{~cm}$. Because the time interval corresponds to twice the distance from the position of the pulse generation to the reflection point, the point of reflection corresponds to about $3 \mathrm{~cm}$ from the detector active area. This is the transition from the microstrip to the SMA connector.

The characteristics of the two detector systems studied in these experiments are listed in Table 2.3.2. All of the diamond used had resistivities greater than $10^{10} \Omega$-cm and had nominal nitrogen concentrations of $25 \mathrm{ppm}$ as quoted by the supplier. As shown in Fig. 2.2.2, a typical I-V curve from the detectors is linear. It must be stressed, however, that this linearity does not characterize the electrical contacts as injecting or non-injecting because linear I-V curve can also result from large surface recombination velocities [25], thereby, eliminating any space charge build up at the contacts. 


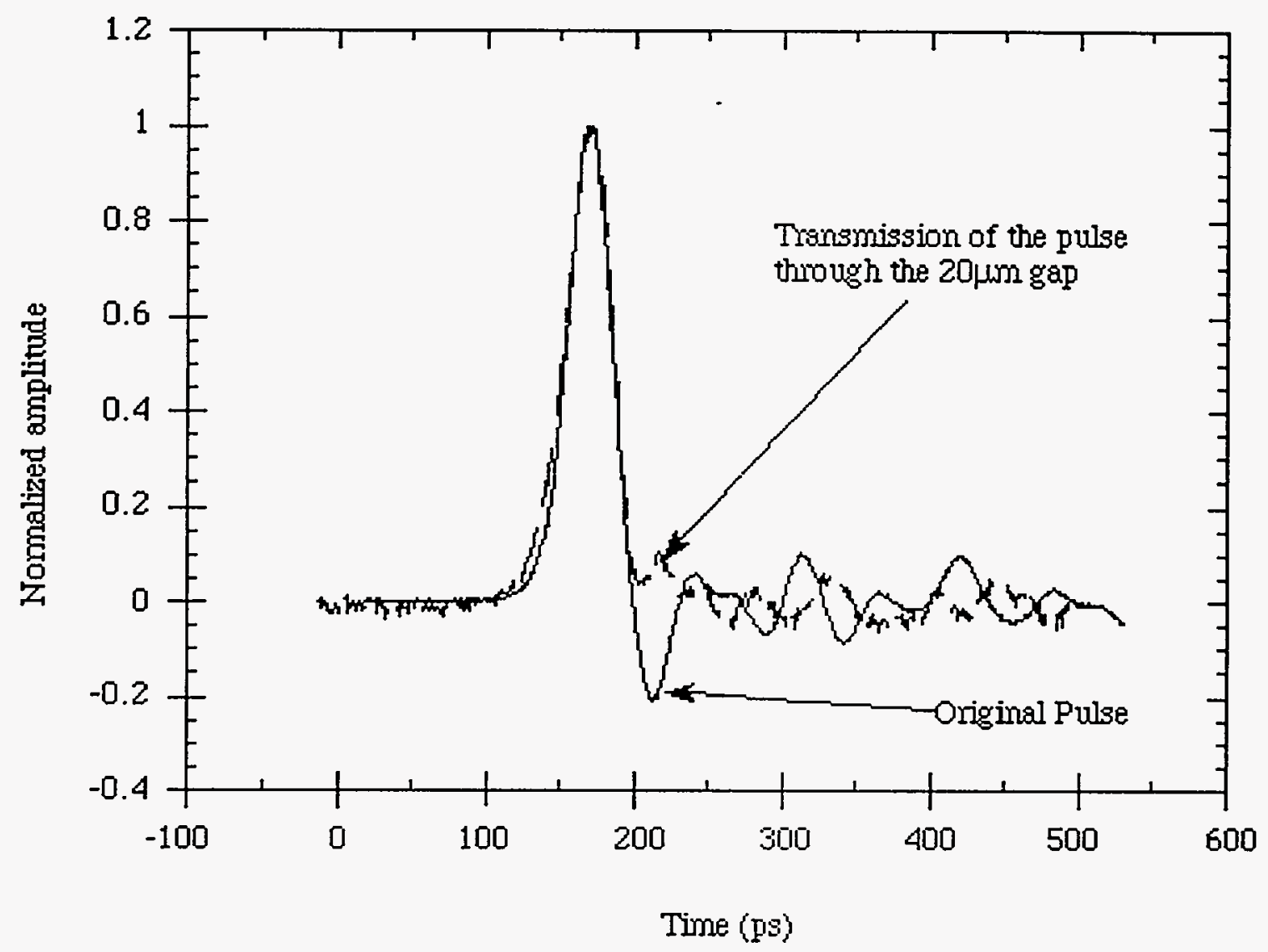

Figure 2.3.4: Comparison of the waveforms obtained after differentiating a steppulse of 20-ps rise time, and after transmitting the step pulse through the microstrip. 


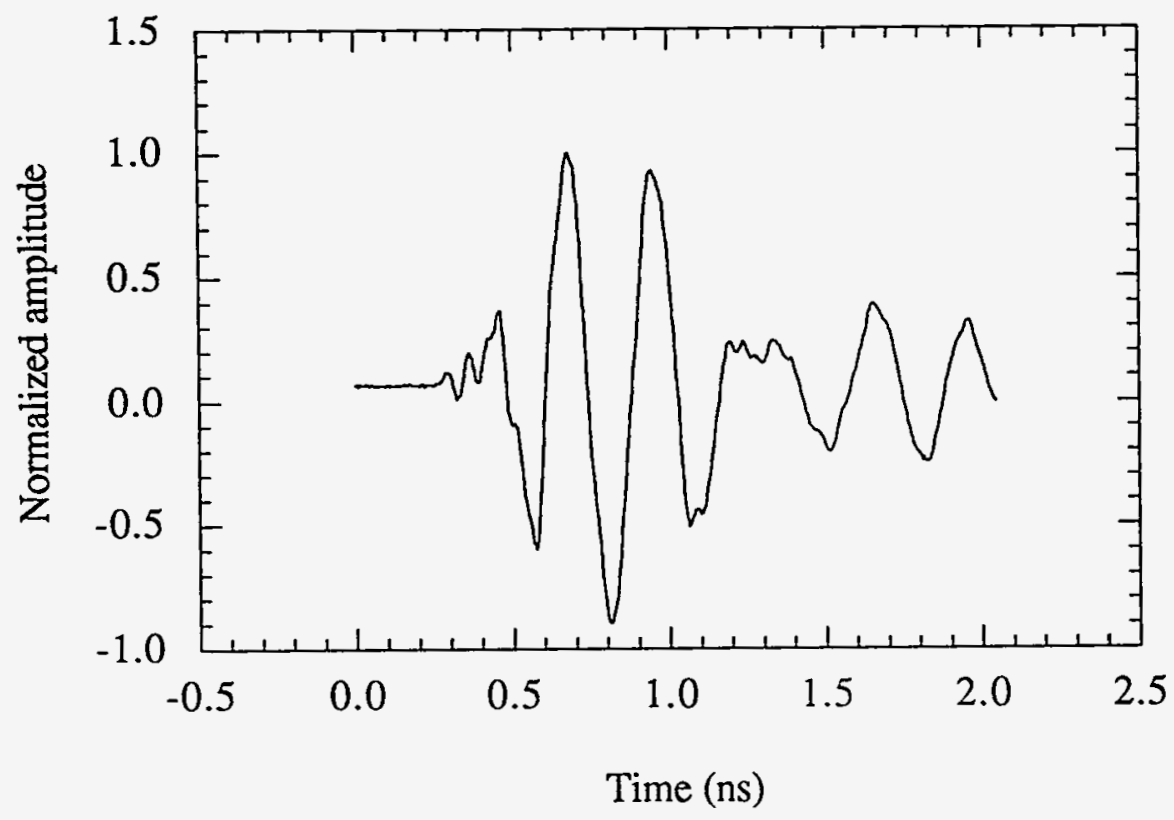

Figure 2.3.5: The transmitted pulse with the same input pulse used to obtain Fig. 2.3.4 


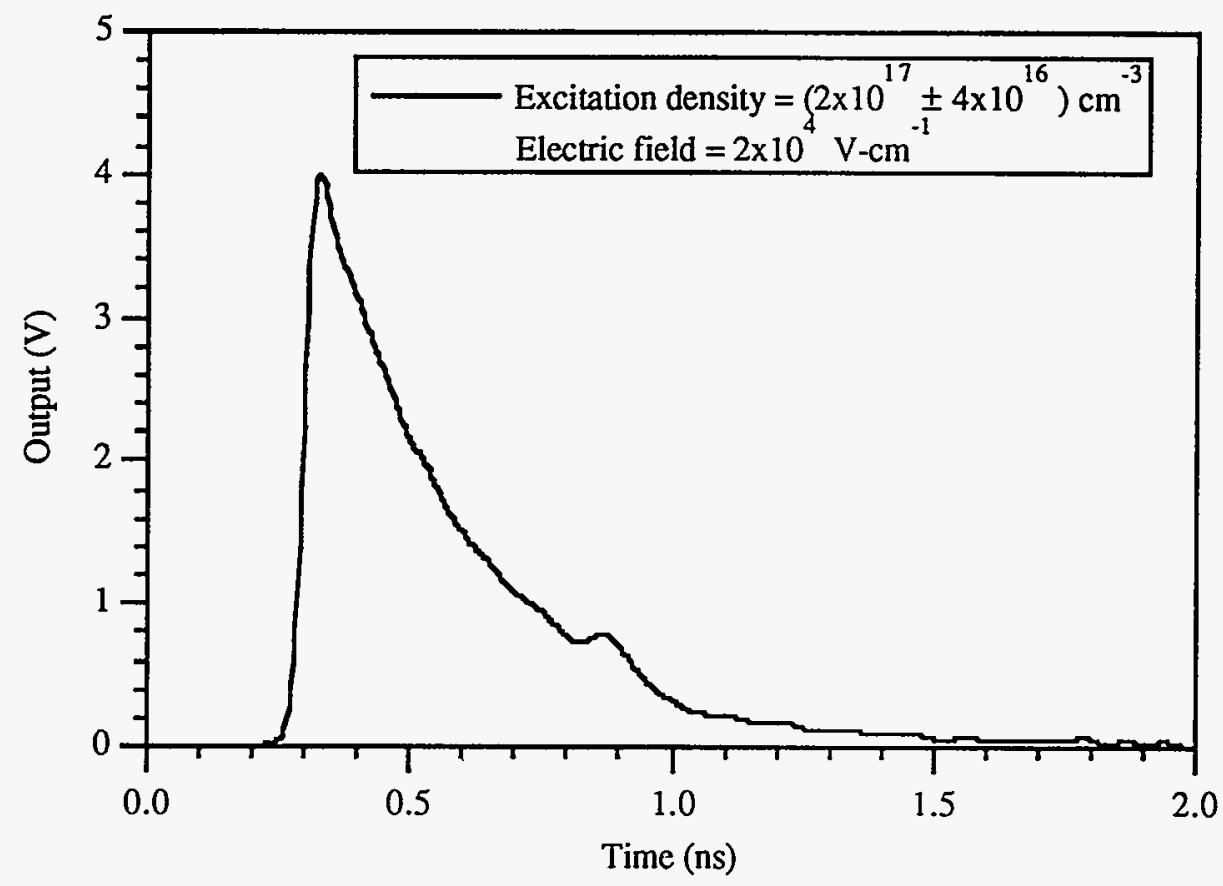

Figure 2.3.6: An example of a transient photoconductive signal taken with a 6-ps UV laser excitation of one of the detectors used in the timeresolved experiments. The active detector area was $20 \mu \mathrm{m}$ by 250 $\mu \mathrm{m}$.

Table 2.3.2: Characteristics of detectors used in the time-resolved measurements.

\begin{tabular}{|c|c|c|c|c|c|}
\hline $\begin{array}{c}\text { Detector } \\
\text { designation }\end{array}$ & $\begin{array}{l}\text { Total detector } \\
\text { surface area } \\
(\mathrm{mm} \times \mathrm{mm})\end{array}$ & $\begin{array}{c}\text { Active } \\
\text { detector area } \\
(\mu \mathrm{m} \times \mu \mathrm{m})\end{array}$ & $\begin{array}{c}\text { Detector } \\
\cdot \\
\text { thickness } \\
(\mu \mathrm{m})\end{array}$ & $\begin{array}{c}\text { Detector } \\
\text { gap width } \\
(\mu \mathrm{m})\end{array}$ & $\begin{array}{c}\text { Detector } \\
\text { resistivity } \\
(\Omega-\mathrm{cm})\end{array}$ \\
\hline HE6.4 & $2 \times 2$ & $20 \times 250$ & 25 & 20 & $>10^{10}$ \\
\hline SI6.9 & $2 \times 2$ & $20 \times 250$ & 26 & 20 & $>10^{10}$ \\
\hline
\end{tabular}




\subsubsection{Experimental arrangement}

The approach used to measure the response of a diamond RIC detector to singleparticle excitation centered around the use of a Hypres model PSP750 high-bandwidth random sampling oscilloscope, a $70 \mathrm{GHz}$ oscilloscope that utilizes a built-in superconducting, near dispersion- and loss-free delay line that enabled signal levels as small as $2 \mathrm{mV}$ into $50 \Omega$ to be sampled directly. Because the oscilloscope was a sampling oscilloscope, many input waveforms were needed to "build" an average output waveform. The total time window set by the oscilloscope was limited to $600 \mathrm{ps}$.

A schematic of the experimental apparatus is shown in Fig. 2.3.7 and is similar to that used by Wagner et al. [26]. Photographs of the actual chamber used are shown in Fig. 2.3.8. An ion beam of either $5-\mathrm{MeV} \mathrm{He}^{+}$or $10-\mathrm{MeV} \mathrm{Si}^{3+}$ from a tandem electrostatic accelerator was elastically scattered by a $400 \AA$ thick platinum foil. Scattered ions that passed through a $20-\mu \mathrm{m}$ diameter aperture in a platinum foil were directed toward the detector surface. Ion trajectories were normal to the detector surface and thus normal to the applied electric field. The event rate in the detector was controlled by varying both the beam current and the scattering angle of the primary beam.

The detector package was located on an X-Y-Z motorized stage to allow alignment of the transmitted beam with the active region of the diamond surface. Signal and other electrical connections were made through a $3.5 \mathrm{~mm}$ diameter, semi-rigid copper microwave coaxial cable. Direct connection to the input of the PSP750 was made with a total cable length of $30 \mathrm{~cm}$. The total rise time of the PSP750-based recording system including the cable, connectors, and the microstrip itself, was measured by propagating either 20-ps Gaussian or step pulses from a Hypres external pulse generator. In Fig. 2.3.9, the output pulse waveform is shown without the detector package in place. The rise time was determined to be $16.5 \pm 0.5 \mathrm{ps}$. With the detector package in place (Fig. 2.3.10), the rise time was determined to be $18.6 \pm 0.6 \mathrm{ps}$. From these measurements, the total rise time for the detector-package was found to be $8.6 \pm 0.4 \mathrm{ps}$ as compared to the 
calculated value of $5 \mathrm{ps}$ (see Sec. 2.3.1). Considering that the measured value also includes any rise-time degradation from discontinuities and impedance mismatches along the microstrip, the agreement can be considered to be reasonable.

Because the microstrip is double-ended, a standard, pulse-counting spectroscopy system could also be connected to the microstrip. This permitted monitoring of pulse amplitudes and intensities during system set up, beam alignment procedures, and during experiments. Bias voltage to the detector was applied through this apparatus because the addition of a bias-tee between PSP750 and the microstrip would degrade the speed of the recording system. 


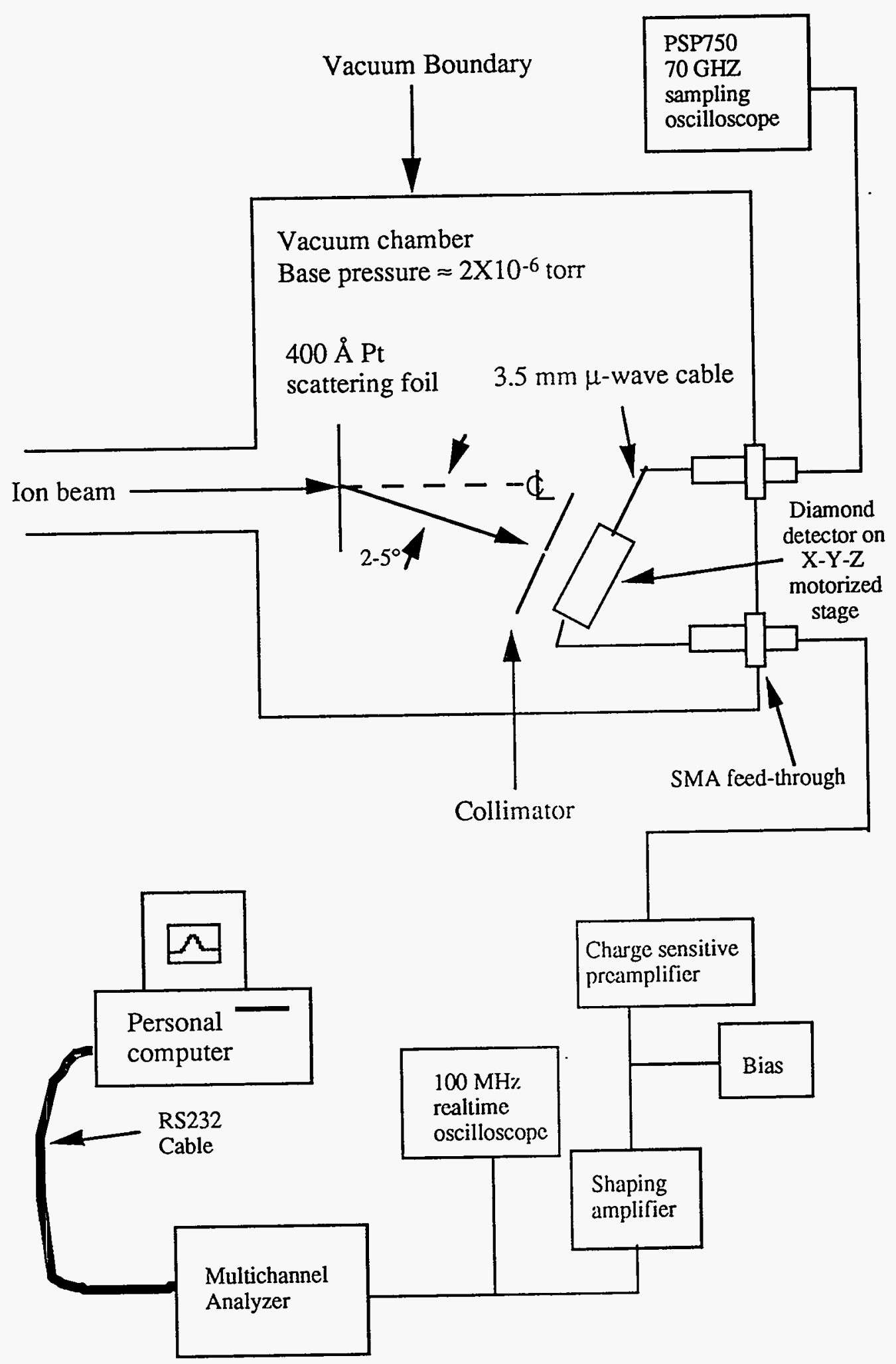

Figure 2.3.7: A schematic for the time-resolved experimental arrangement. 
A)

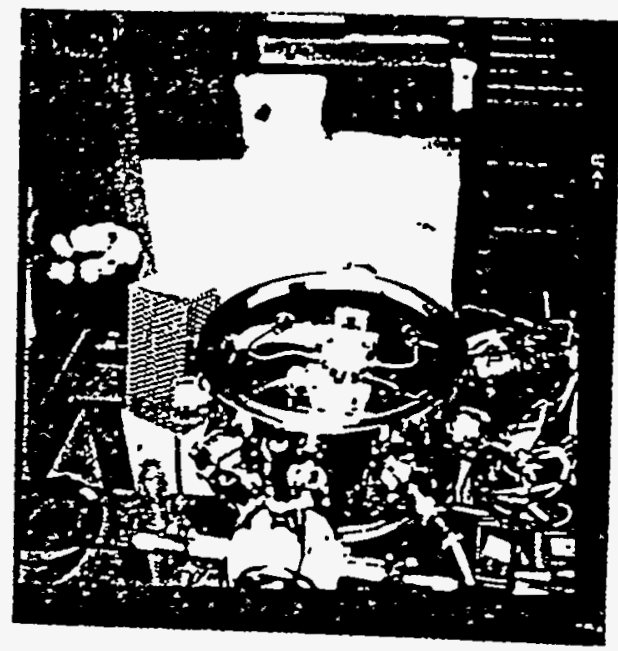

B)

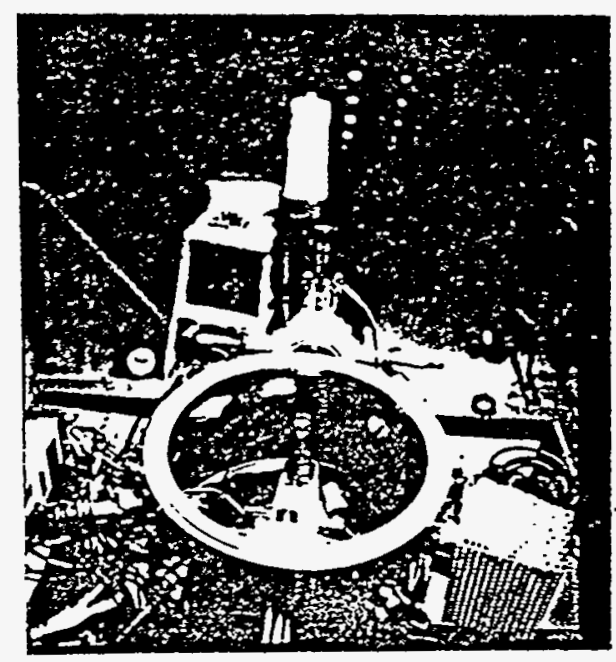

Figure 2.3.8: Photographs of the vacuum chamber and its attachments; $A$ ) the ion-beam entrance is at the bottom of the photograph, looking toward the mounted detector, B) a view from the direction opposite to A), looking toward the Pt-scattering foil. 


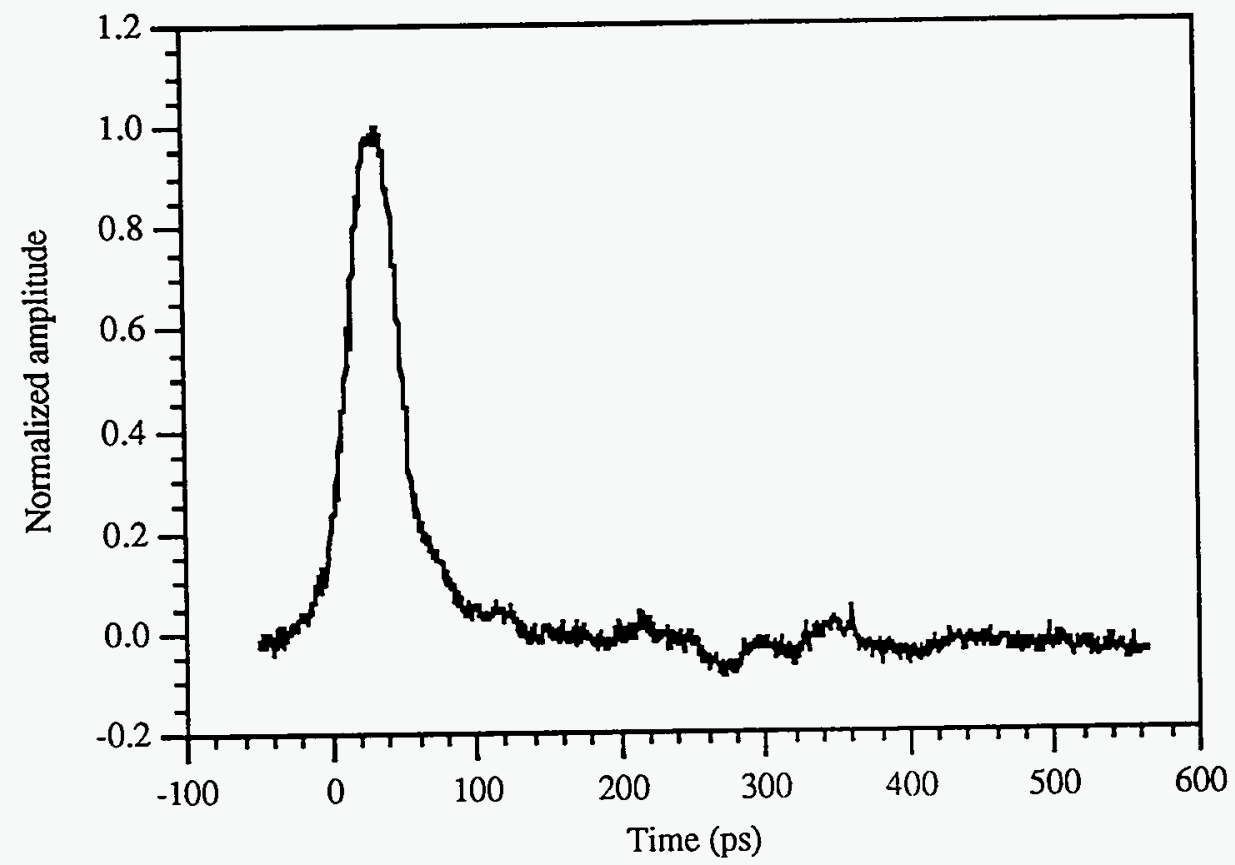

Figure 2.3.9: The output pulse after propagating a $20 \mathrm{ps}$ Gaussian pulse through all of the cables and connectors excluding the detector package.

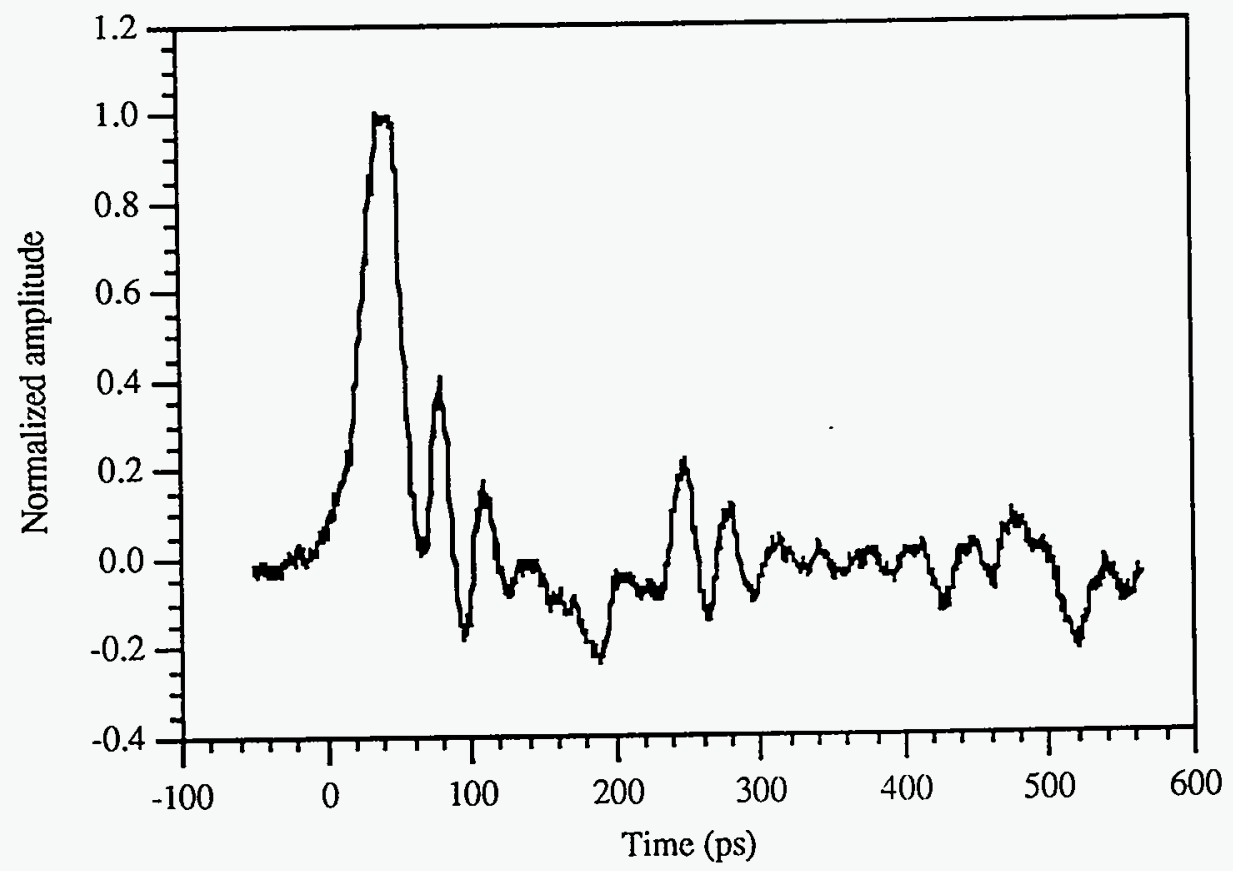

Figure 2.3.10: The output pulse after propagating a 20-ps step pulse through all of the cables and connectors including through the gap on the detector. 


\section{References}

[1] DubbleDee Harris Corp., 100 Stierli Court, Mount Arlington, New Jersey 07856 (U. S. A.)

[2] L. S. Pan, S. Han, D. R. Kania and K. Okano, Diamond Films and Tech., 2(2) (1992) 99

[3] S. A. Grot, G. Sh. Gildenblat, C. W. Hatfield, C. R. Wronski, A. R. Badzian, T. Badzian and R. Messier, IEEE Elec. Dev. Lett, 11(2) (1990) 100

[4] S. A. Grot, S. Lee, G. Sh. Gildenblat, C. W. Hatfield, C. R. Wronski, A. R. Badzian, T. Badzian and R. Messier, J. Mater. Res., 5(11) (1990) 2497

[5] Y. Mori, H. Kawarada and A. Hiraki, Appl. Phys. Lett., 58(9) (1991) 940

[6] W. Kern and D. A. Puotinen, RCA Rev., June 1970, 187.

[7] K. L. Moazed, J. R. Zeidler and M. J. Taylor, J. Appl Phys., 68(5) (1990) 2246

[8] L. Holland, in Science and Technology of Surface Coating, Ed. B. N Chapman and J. C. Anderson, Academic Press (1974) 384

[9] T. Moeller, Inorganic Chemistry: an Advanced Textbook, John Wiley \& Sons, 696-700 (1952).

[10] H. J. Emeléus, J. S. Anderson, Modern Aspects of Inorganic Chemistry, D. Van Nostrand Co., Inc., (1938).

[11] F. Ephraim, Inorganic Chemistry, Nordeman Publishing Co., Inc., 848-856 (1943).

[12] J. F. Ziegler, H. H. Andersen and J. Biersack, TRIM-90.5 Computer Simulation Code, Transport of Ions in Matter (1990).

[13] G. F. Knoll, Radiation Detection and Measurement, John Wiley \& Sons, 49 (1979) .

[14] The silicon detector used for the pulse-height calibration was supplied by EG\&G Ortec. 
[15] See for example; C. Canali, E. Gatti, S. F. Kozlov, P. F. Manfredi, C.

Manfredotti, F. Nava and A. Quirini, NIM, 160 (1979) 73

[16] J. N. Demas, Excited State Lifetime Measurements, Academic Press, New York, 1983

[17] C. H. Lee, Picosecond Optoelectronic Devices, New York, Academic Press (1984)

[18] D. H. Auston, "Picosecond photoconductivity: high-speed measurements of devices and materials", in Measurement of High-Speed Signals in Solid State Devices, Edited by R. B. Marcus, Vol. 28, Academic Press, Boston (1990) 85

[19] L. S. Pan, Ph. D thesis, Stanford University (1991)

[20] I. J. Bahl and R. Garg, Proc. IEEE, 65 (11) (1977) 1611

[21] J. F. Whitaker et al., IEEE MTT, 35-1(1987).

[22] K. C. Gupta, R. Garg and I. J. Bahl, Microstrip Lines and Slotlines, Artech House, Dedham, MA (1979) 89

[23] M. Maeda, IEEE MTT, 20(6) (1972) 390

[24] L. S. Pan, D. R. Kania, P. Pianetta and O. L. Landen, Appl. Phys. Lett., 57(6) (1990) 623

[25] E. H. Rhoderick and R. H. Williams, Metal-Semiconductor Contacts, second edition, Oxford science publications, 1988; H. K. Henisch, Semiconductor contacts: an Approach to Ideas and Models, Oxford science publications, 1989

[26] R. S. Wagner, N. Bordes, J. Bradley, C. Maggiore, A. Knudson and A. Campbell, IEEE Trans. Nuc. Sci., NS-35 (1988) 1578. 


\section{Chapter 3: Integrated-charge experiments}

In this chapter, results from the integrated-charge experiments are discussed and the model used to interpret data from these are presented. The experiments were conducted with the experimental arrangement described in Sec. 2.2. Given the experimental results, the charge collection characteristics can be fitted to theoretical descriptions in an attempt to estimate such material characteristics as the electron (hole) mobility, $\mu_{e}\left(\mu_{h}\right)$, and lifetime, $\tau_{e}\left(\tau_{h}\right)$, the mean energy required to produce an electronhole pair and device characteristics such as the description of the boundary at metal/diamond interface. Furthermore, we have modeled not only the charge collection characteristics of a detector but also the detector resolution from $\alpha$-bombardment in an attempt to obtain further insight into the charge collection characteristics of the detectors.

\subsection{Experimental results}

By using the experimental arrangement described in section 2.2, we obtained pulse-height spectra as a function of applied electric field by irradiating the detector with $\alpha$-particles from the ${ }^{241} \mathrm{Am}$ source. Three detectors with varying thickness were studied (see Table 2.1.1). For brevity, we will refer to a detector by the designation given in that table. Table 3.1.1 lists the pulse-height calibrations used to convert channel numbers to collected charge for these detectors.

The first set of data was obtained with SYM14m. The bias voltage was varied in the range $\pm(2-200) \mathrm{V}$, corresponding to electric fields in the range $\pm(1.4-140) \mathrm{KV} / \mathrm{cm}$. The polarity indicated is the polarity of the biased electrode (opposite to the electrode through which the $\alpha$-particles entered to reach the diamond) as shown in Fig 2.2.4. A few representative spectra obtained from these measurements are shown in Fig. 3.1.1. Gaussian fits to the spectra are also shown in the figure. The fits to the experimental 
Table 3.1.1: Calibrations used to convert channel number to collected charge for the detectors studied.

\begin{tabular}{|c|c|c|}
\hline $\begin{array}{l}\text { Detector } \\
\text { designation }\end{array}$ & $\begin{array}{l}\text { Slope } \\
\text { (number of carriers collected } \\
\text { per channel) }\end{array}$ & $\begin{array}{c}\text { y-intercept } \\
\text { (number of carriers collected) }\end{array}$ \\
\hline \multirow[t]{2}{*}{ SYM14m } & $1.578 \times 10^{3}$ (“+" polarity) & $-5.379 \times 10^{5}$ ("+" polarity) \\
\hline & $1.581 \times 10^{3}$ (“-"” polarity) & $-5.446 \times 10^{5}$ (“-" polarity) \\
\hline SYM25m & $1.723 \times 10^{3}$ & $-3.060 \times 10^{5}$ \\
\hline SYM70m & $1.723 \times 10^{3}$ & $-3.060 \times 10^{5}$ \\
\hline
\end{tabular}

data appear to describe the distributions well. The quality of the fits will be examined in Sec. 3.1.1.

In all pulse-height spectra, the analyzer noise was truncated by adjusting the pulse-height discriminator level to channel 364. The smallest separation between this noise and the full-energy peak in any of the spectra was about 50 channels. The FWHM of the full-energy peak is about 38 channel-equivalent. Because the separation of the peak and the noise is much greater than the width of the full-energy peak, the influence of the noise on the width of the peak is negligible.

All of the spectra at varying applied electric field show a degree of low- and highenergy tailing. At low fields (Fig. 3.1.1-A and -B), the spectra are nearly symmetric about the centroid. At low fields (below about $10 \mathrm{KV} / \mathrm{cm}$ ), the carrier mobility is constant as a function of field. Therefore, the carrier velocity is linearly proportional to the electric field leading to the magnitude of collected charge being linearly proportional 
A)

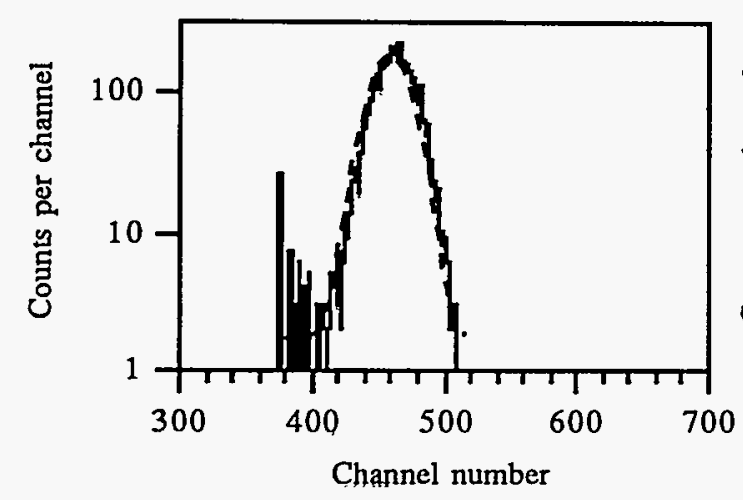

C)

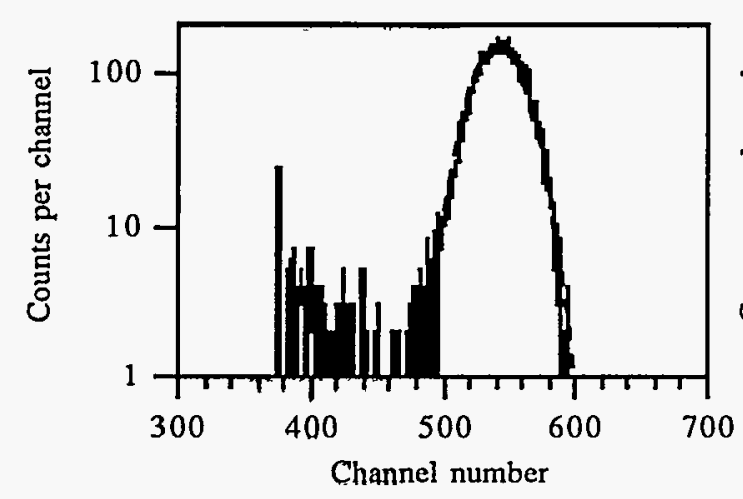

B)

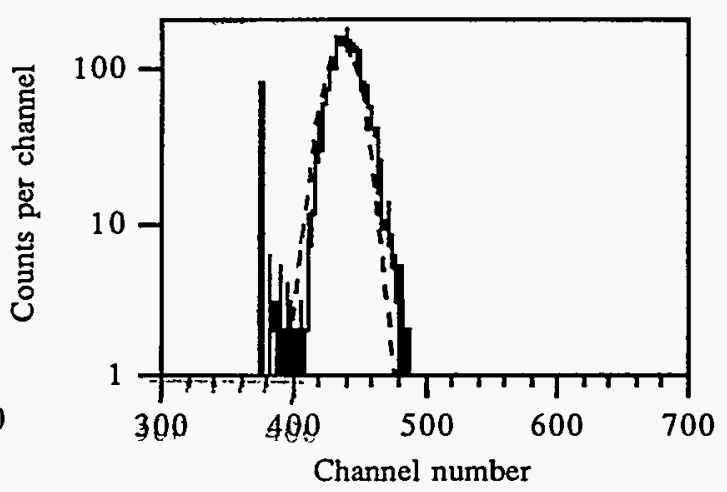

D)

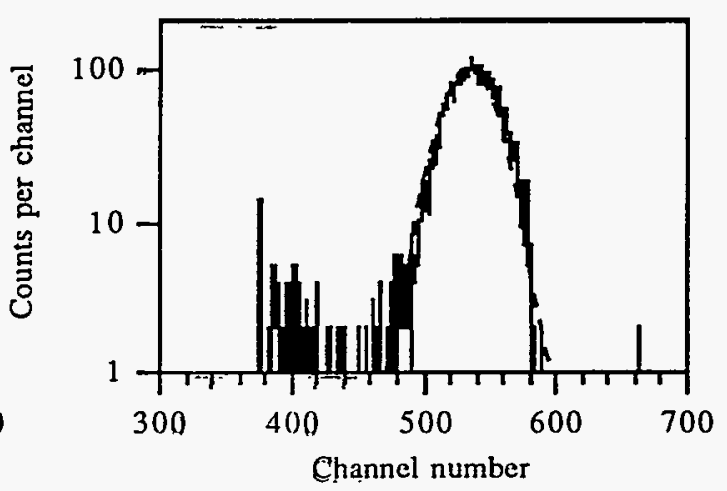

Figure 3.1.1: Pulse-height spectra for detector SYM14m as a function of bias voltage, $\mathrm{V}_{\mathrm{b}}$. Solid line $\left(-\frac{-}{-}\right)$ is the experimental data and dashed line (---) is the Gaussian fit to the data. A) $V_{b}=+10 \mathrm{~V}(6.9$ $\left.\mathrm{KV} / \mathrm{cm}) . \mathrm{B}) \mathrm{V}_{\mathrm{b}}=-10 \mathrm{~V}(6.9 \mathrm{KV} / \mathrm{cm}) . \mathrm{C}\right) \mathrm{V}_{\mathrm{b}}=+100 \mathrm{~V}(69$ $\mathrm{KV} / \mathrm{cm})$. D) $\mathrm{V}_{\mathrm{b}}=-100 \mathrm{~V}(69 \mathrm{KV} / \mathrm{cm})$.

to the electric field assuming that the carrier lifetime is constant. Thus, in this regime the fluctuations in the mean free path of a carrier would lead to equal fluctuations in pulse height leading to a symmetric pulse height distribution [1]. At high fields (Fig. 3.1.1-C and -D), the spectra are slightly skewed toward the higher energies. The low energy tails are usually an indication of carrier trapping leading to incomplete charge collection. The high energy tails occur in all cases since the charge collection is a statistical process. 
Next, we replaced SYM14m with SYM25m and obtained pulse-height spectra as a function of bias voltage. The bias voltage was varied in the range $\pm(10-300) \mathrm{V}$, corresponding to electric fields in the range $\pm(4 \mathrm{KV} / \mathrm{cm}-120 \mathrm{KV} / \mathrm{cm})$. A few typical spectra along with the fits to Gaussian distribution function are shown in Fig. 3.1.2 The fits appear to describe the pulse-height distributions reasonably well. Again, small skewing toward the high-energy side is apparent at the higher electric field at negative bias polarity (Fig. 3.1.2-C). A difference in the centroid position for the two bias polarities can be easily seen. The asymmetry in the centroid positions is thought to be due to the difference in the electrical properties of electrons and holes. This asymmetry will be further examined in Sec. 3.2.

Finally, we performed the same measurements with SYM70m. Applied bias voltage was varied in the range $\pm(110-900) \mathrm{V}$, corresponding to electric fields in the range $\pm(16 \mathrm{KV} / \mathrm{cm}-130 \mathrm{KV} / \mathrm{cm})$. Again, a few characteristic spectra are shown in Fig. 3.1.3. As we have done with the other two detectors, the fits to a Gaussian are shown along with the measurements. Consistent with the other two, the fits appear to describe the distributions reasonably well. With this detector, however, skewing toward the highenergy side is negligible. The pulse-height spectra are similar to those for SYM25m, with the exception that the centroid positions between spectra taken with positive and negative polarities are quite large. 
A)

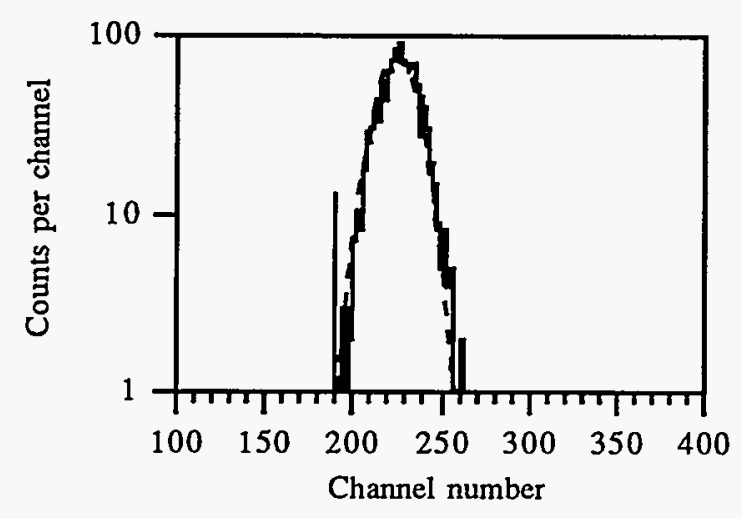

C)

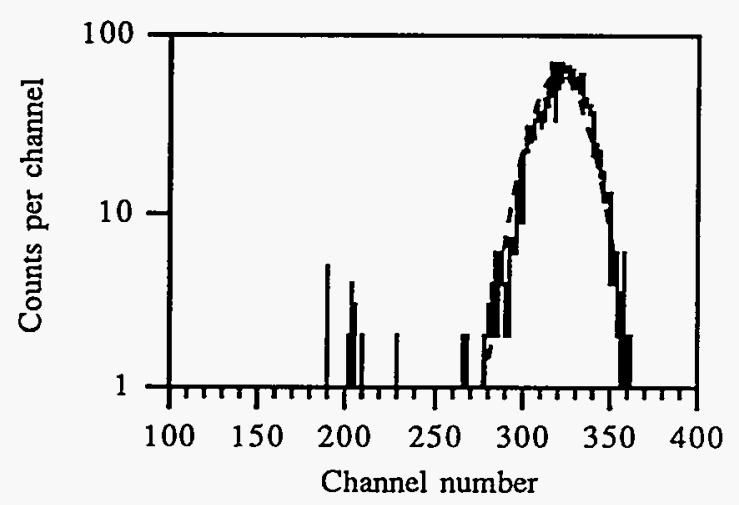

B)

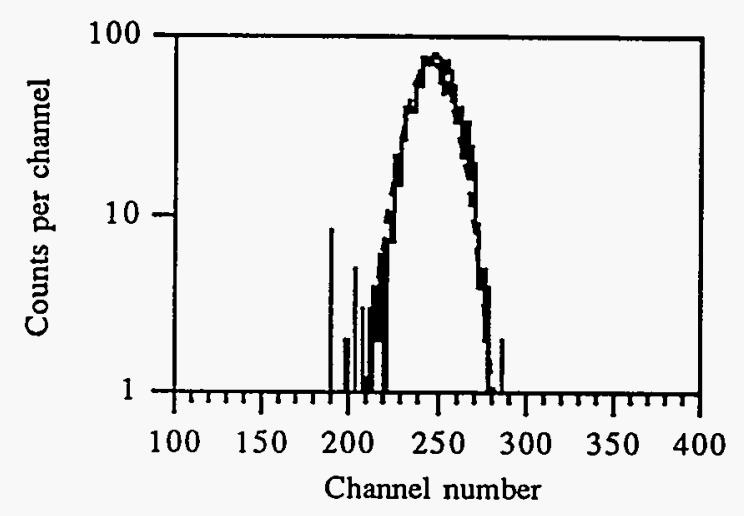

D)

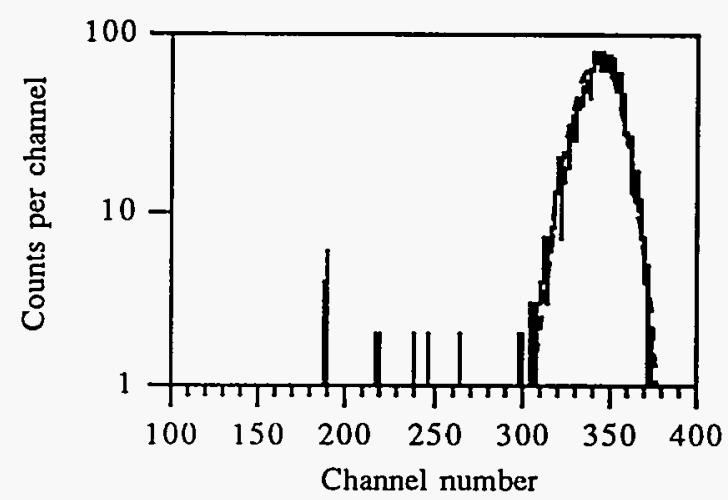

Figure 3.1.2: Pulse-height spectra for detector SYM25m as a function of bias voltage, $\mathrm{V}_{\mathrm{b}}$. Solid line $(-)$ is the experimental data and dashed line (---) is the Gaussian fit to the data. A) $V_{b}=+20 \mathrm{~V}(8$ $\left.\mathrm{KV} / \mathrm{cm}) . \mathrm{B}) \mathrm{V}_{\mathrm{b}}=-20 \mathrm{~V}(8 \mathrm{KV} / \mathrm{cm}) . \mathrm{C}\right) \mathrm{V}_{\mathrm{b}}=+200 \mathrm{~V}(80$ $\mathrm{KV} / \mathrm{cm}) . \mathrm{D}) \mathrm{V}_{\mathrm{b}}=-200 \mathrm{~V}(80 \mathrm{KV} / \mathrm{cm})$. 
A)

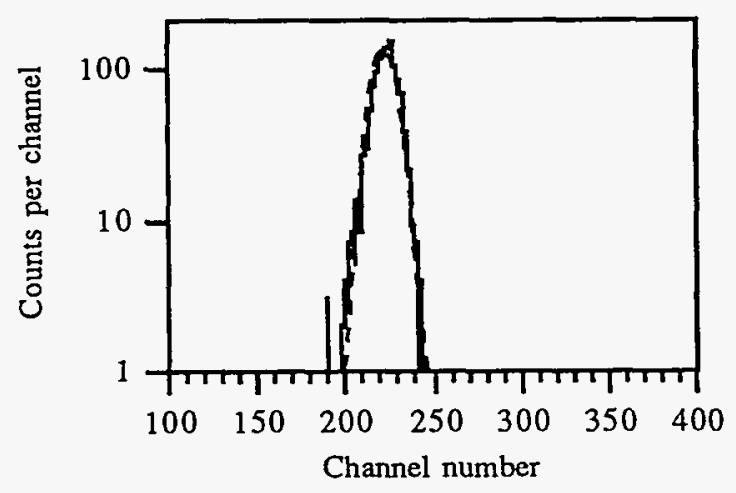

C)

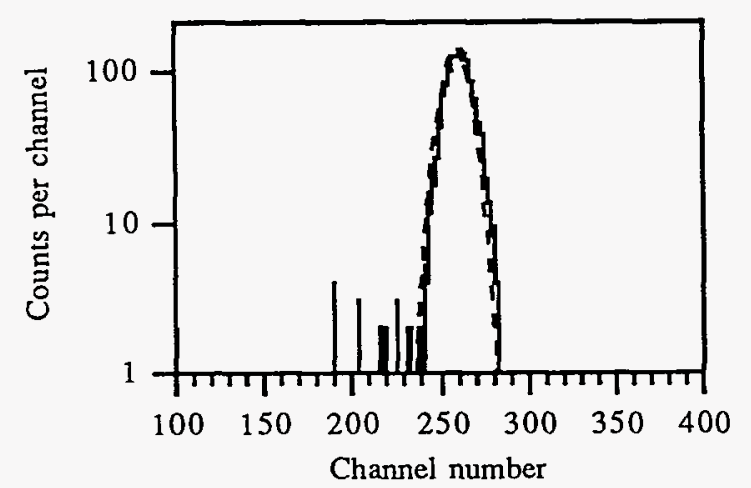

B)

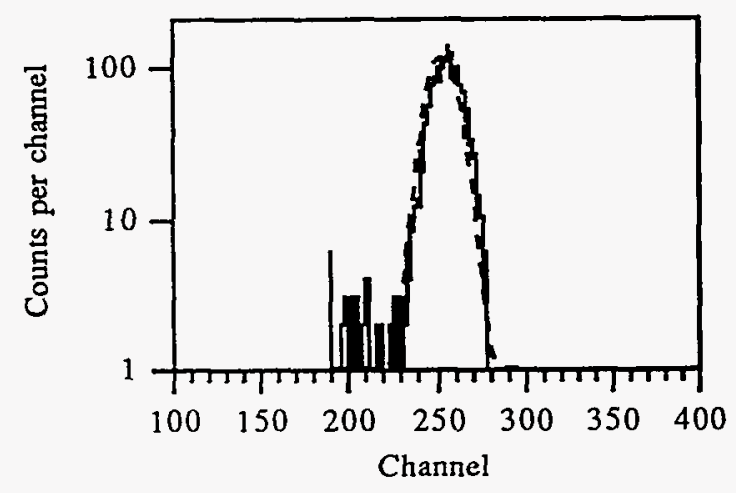

D)

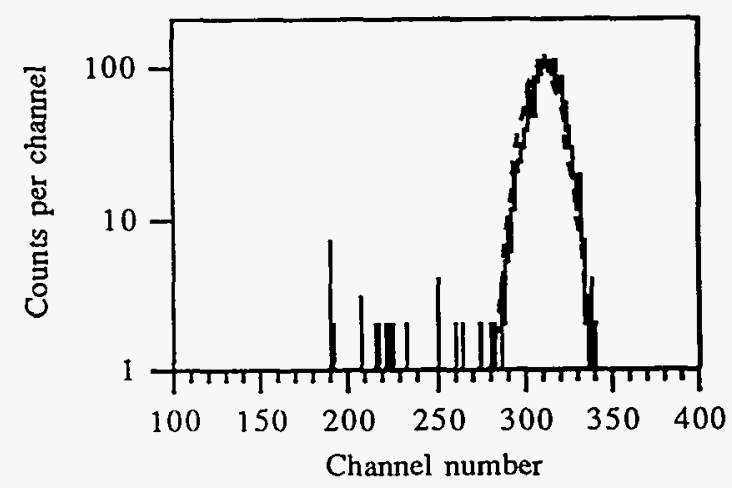

Figure 3.1.3: Pulse-height spectra for detector SYM70m as a function of bias voltage, $\mathrm{V}_{\mathrm{b}}$. Solid line (-) is the experimental data and dashed line (---) is the Gaussian fit to the data. A) $V_{b}=+150 \mathrm{~V}(21$

$\left.\mathrm{KV} / \mathrm{cm}) . \mathrm{B}) \mathrm{V}_{\mathrm{b}}=-150 \mathrm{~V}(21 \mathrm{KV} / \mathrm{cm}) . \mathrm{C}\right) \mathrm{V}_{\mathrm{b}}=+600 \mathrm{~V}(86$

$\mathrm{KV} / \mathrm{cm}) . \mathrm{D}) \mathrm{V}_{\mathrm{b}}=-600 \mathrm{~V}(86 \mathrm{KV} / \mathrm{cm})$.

\subsubsection{Analysis of experimental results}

In each spectrum, three pieces of information are provided that can give insight into detector characteristics. The first is the centroid position of the full-energy peak. This defines the total mean collected charge and can be used to estimate electrical properties by modeling the physical processes involved in the charge collection. The second and third, the FWHM of the full-energy peak and the actual shape of the spectral 
distribution, are closely related and provide information on the effects of incomplete charge collection.

The total mean collected charge and the detector resolution were estimated by fitting a spectrum to a simple Gaussian distribution function using a nonlinear leastsquares method. As we have already seen from Fig. 3.1.1-3.1.3, the measured spectral distribution appears to be well described with the Gaussian distribution function. In Fig. 3.1.4, we show a pulse-height spectrum from ${ }^{241} \mathrm{Am} \alpha$-particles on diamond at an applied electric field of $E=-4 \mathrm{KV} / \mathrm{cm}$. It is clear that the spectrum (Fig. 3.1.4-A) is nearly Gaussian in shape. The quality of the Gaussian fit can be seen in Fig. 3.1.4-B. The error bars shown were calculated from the counting statistics as

$$
\sigma_{N}=\sqrt{N}
$$

where $\mathrm{N}=$ number of counts in the channel

$$
\sigma_{N}=\text { fluctuation in the channel }
$$

Fig. 3.1.5 is a pulse-height spectrum taken at an electric field of $E=-100 \mathrm{KV} / \mathrm{cm}$ along with a Gaussian fit to the data. In this figure, a much greater deviation between the Gaussian fit and the measured spectrum is seen on the high-energy side. However, within errors, the Gaussian fit describes the spectrum reasonably well. As a result, spectral information was obtained by fitting simple Gaussian distributions to all pulseheight spectra for the entire range of applied electric field. The channel locations of the centroid and the widths of the distributions were converted to reflect the collected charge carriers by using the calibration data in Table 3.1.1. 


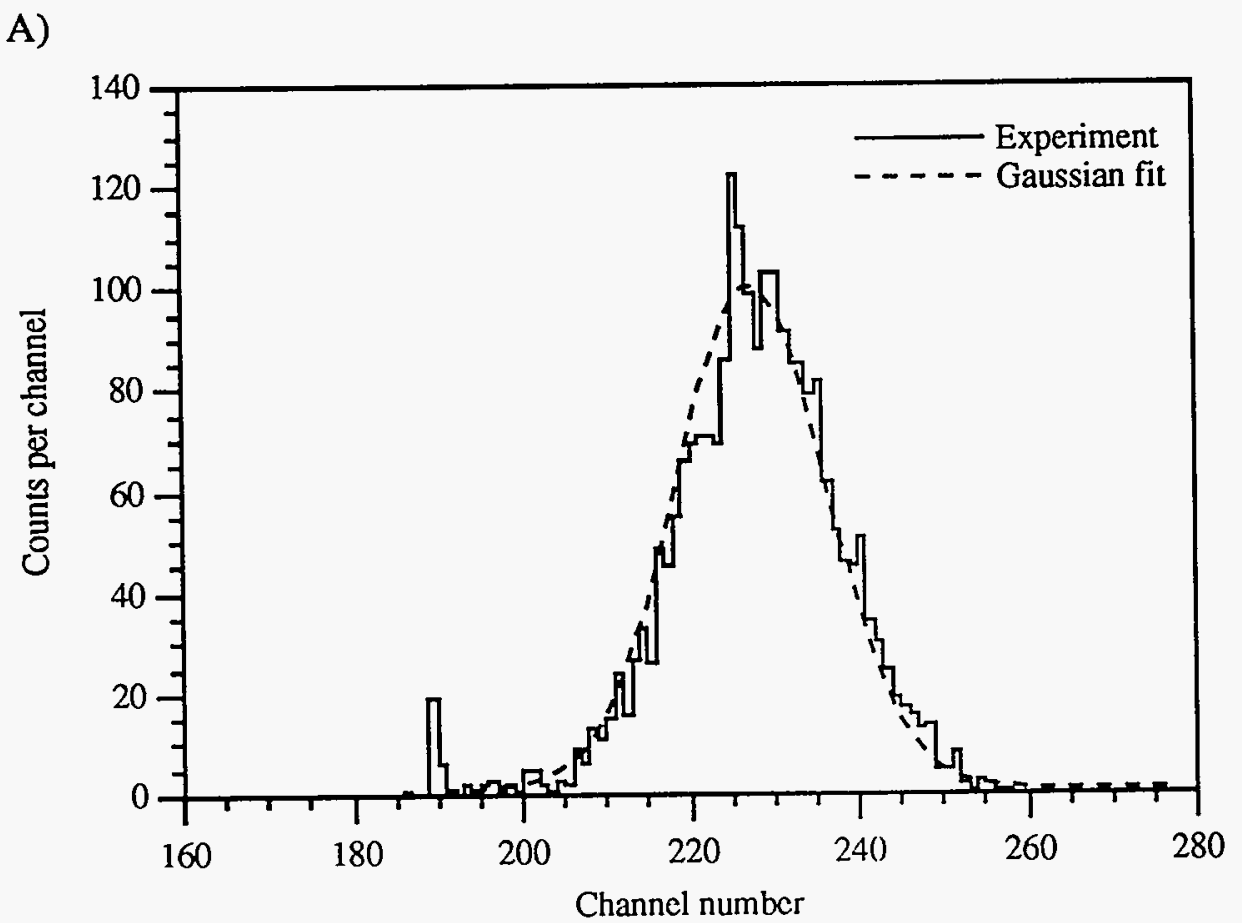

B)

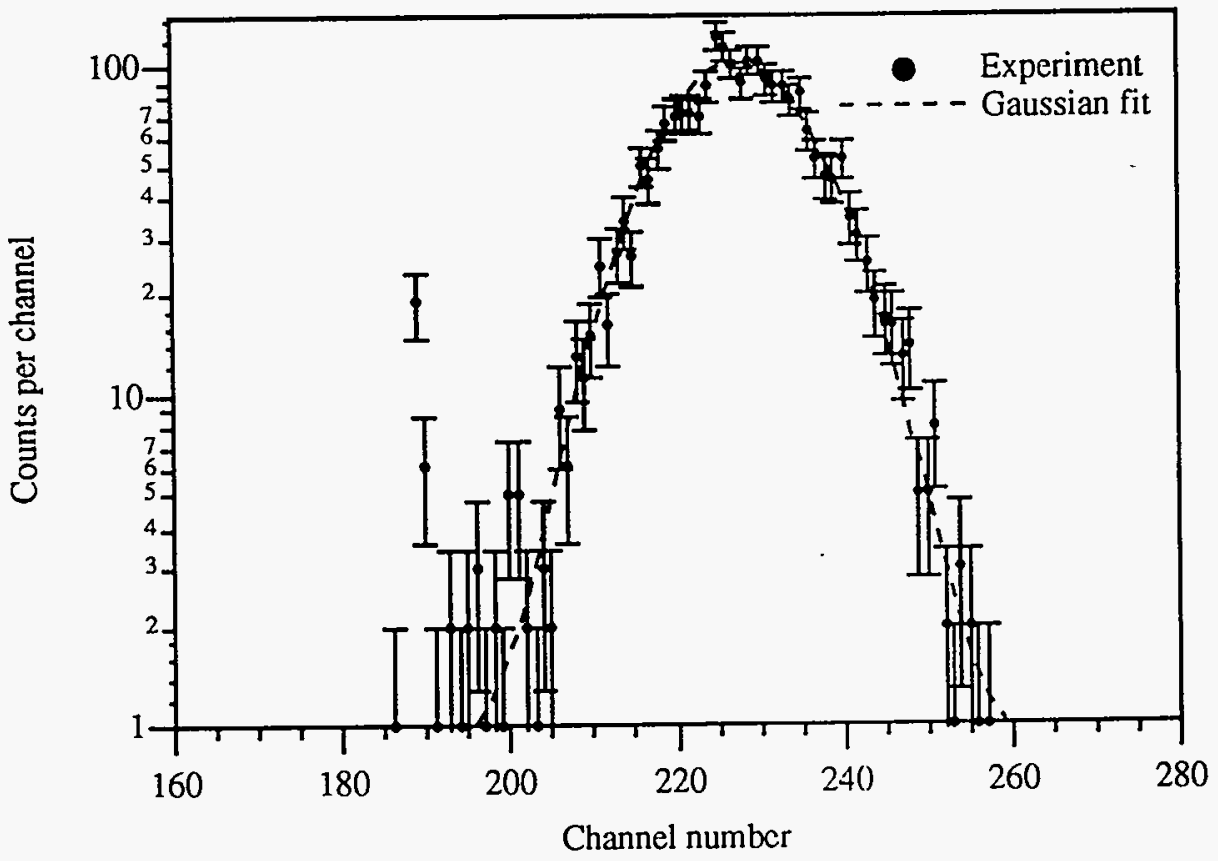

Figure 3.1.4: Pulse-height spectrum from detector SYM25m at an applied electric field of $E=-4 \mathrm{KV} / \mathrm{cm}$. A) is the pulse-height spectrum in linear scales and B) is A) in semi-log scales to emphasize the spectral tail regions. 
A)

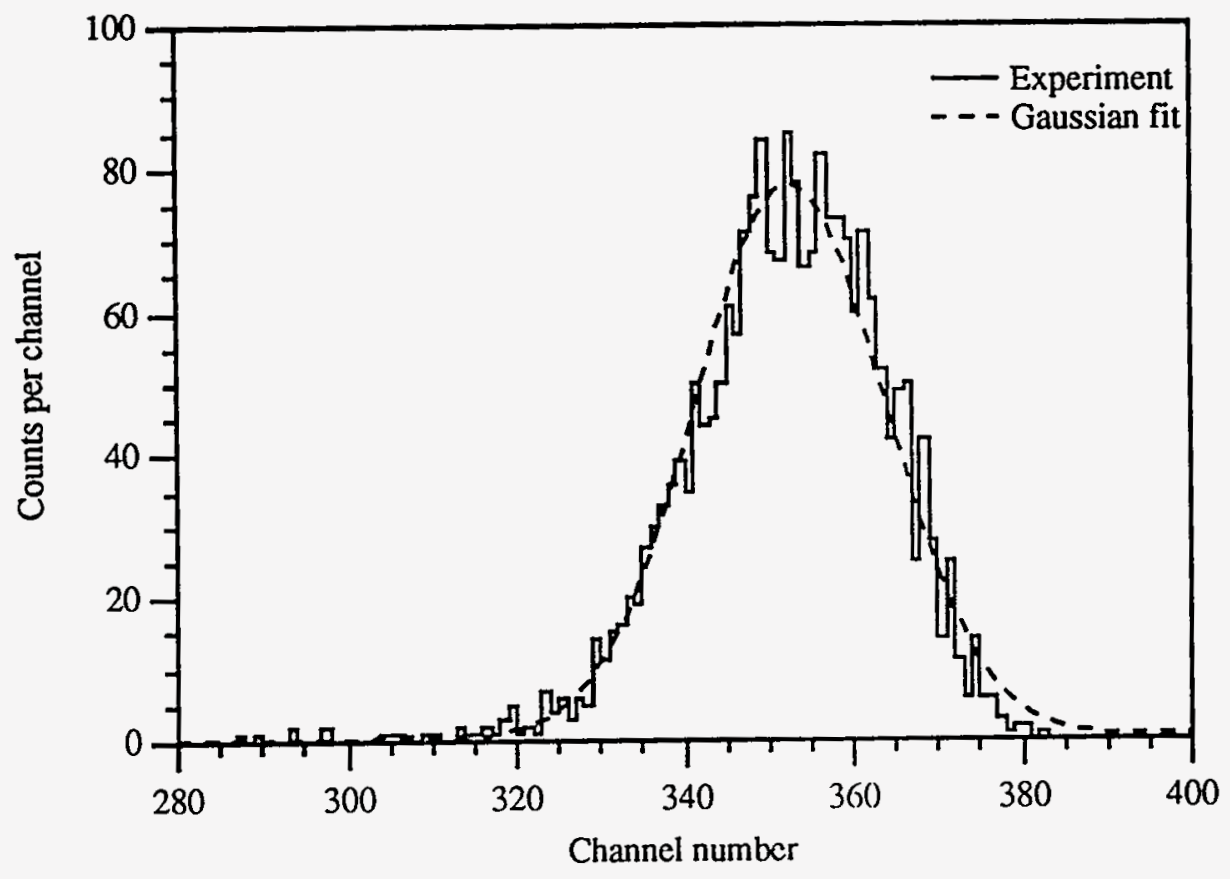

B)

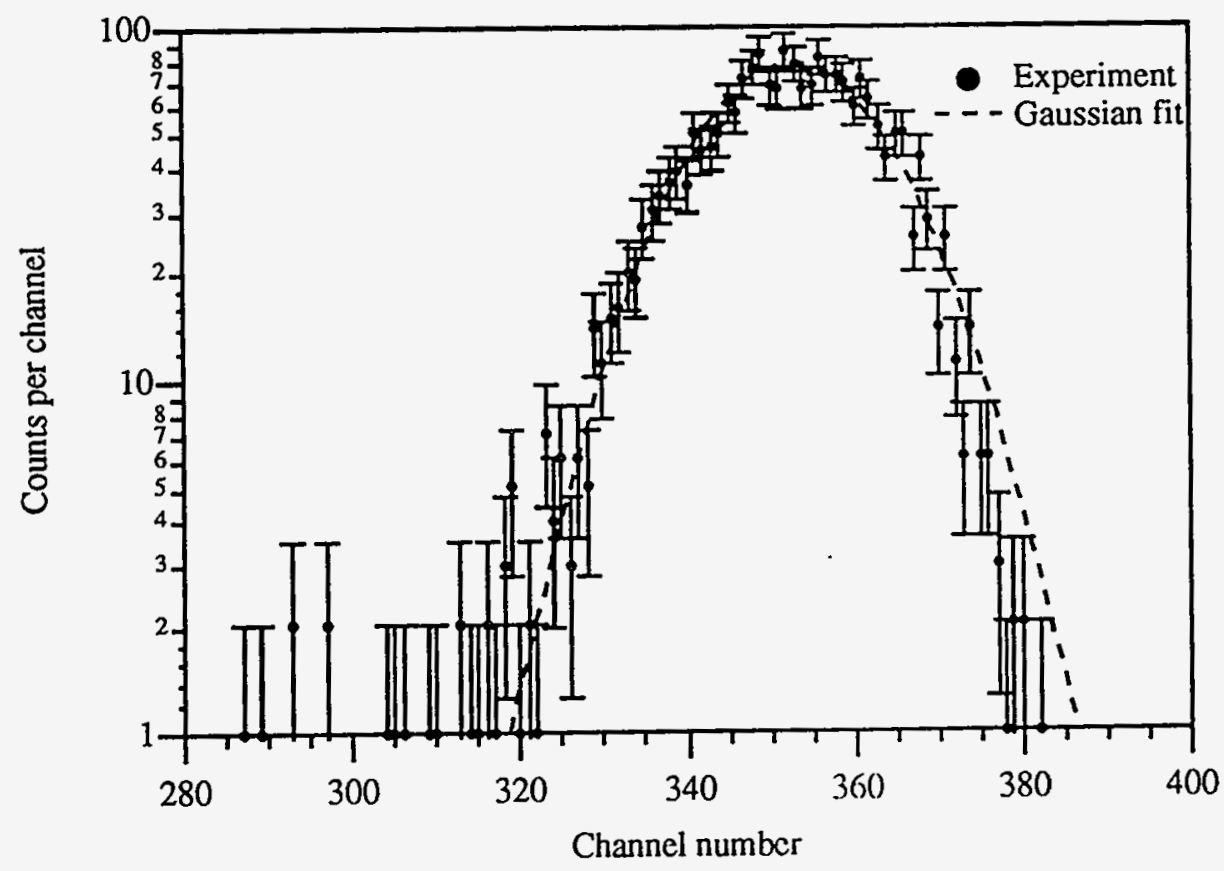

Figure 3.1.5: Pulse-height spectrum from SYM25m at an applied electric field of $E=-100 \mathrm{KV} / \mathrm{cm}$. A) is the pulse-height spectrum in linear scales and B) is A) in semi-log scales to emphasize the spectral tail regions. 
3.1.2 Total mean collected charge results

In Fig. 3.1.6, the total mean collected charge is shown as a function of applied electric field for SYM14m. The profile shown is similar to that first measured and discussed by Hecht [2] using photoconductivity of $\mathrm{AgCl}$ crystals, and later by Mott and Gurney [3] in discussing Gudden and Pohl's data taken with diamond crystals excited with $546 \mathrm{~nm}$ light [4]. For electric fields below $0.1 \times 10^{5} \mathrm{~V} / \mathrm{cm}$, the total collected charge appears to vary linearly with electric field, and is indicative of a mean carrier mobility that is independent of the field. The profile also indicates an approach to saturation in the collected charge at high fields $\left(\geq 0.5 \times 10^{5} \mathrm{~V} / \mathrm{cm}\right)$.

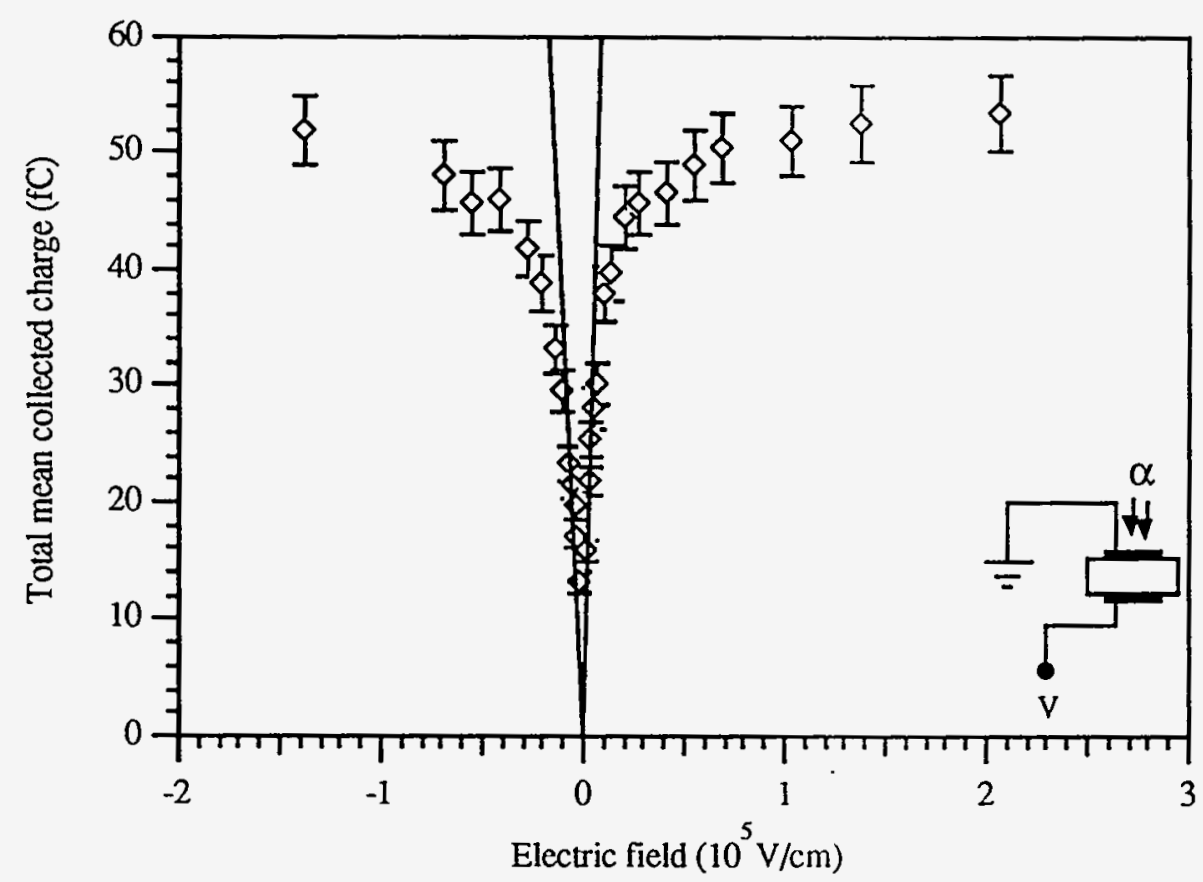

Figure 3.1.6: Total mean collected charge as a function of applied electric field for SYM14m. The solid line (—) is the linear portion of the profile.

The total mean collected charge as a function of electric field for SYM $25 \mathrm{~m}$ is shown in Fig. 3.1.7. Unlike the data shown in Fig. 3.1.6, a clear asymmetry in the 
collected charge with polarity of the field is prominent. This is presumably due to nonuniform excitation of the detector and differences in transport properties of electrons and holes. Kozlov et al. [5] have also seen this type of asymmetry (Fig. 3.1.8) where the ratio of the $\alpha$-particle range to the detector thickness was in the range $0.03-0.1$. For SYM25m, this value was about 0.6 .

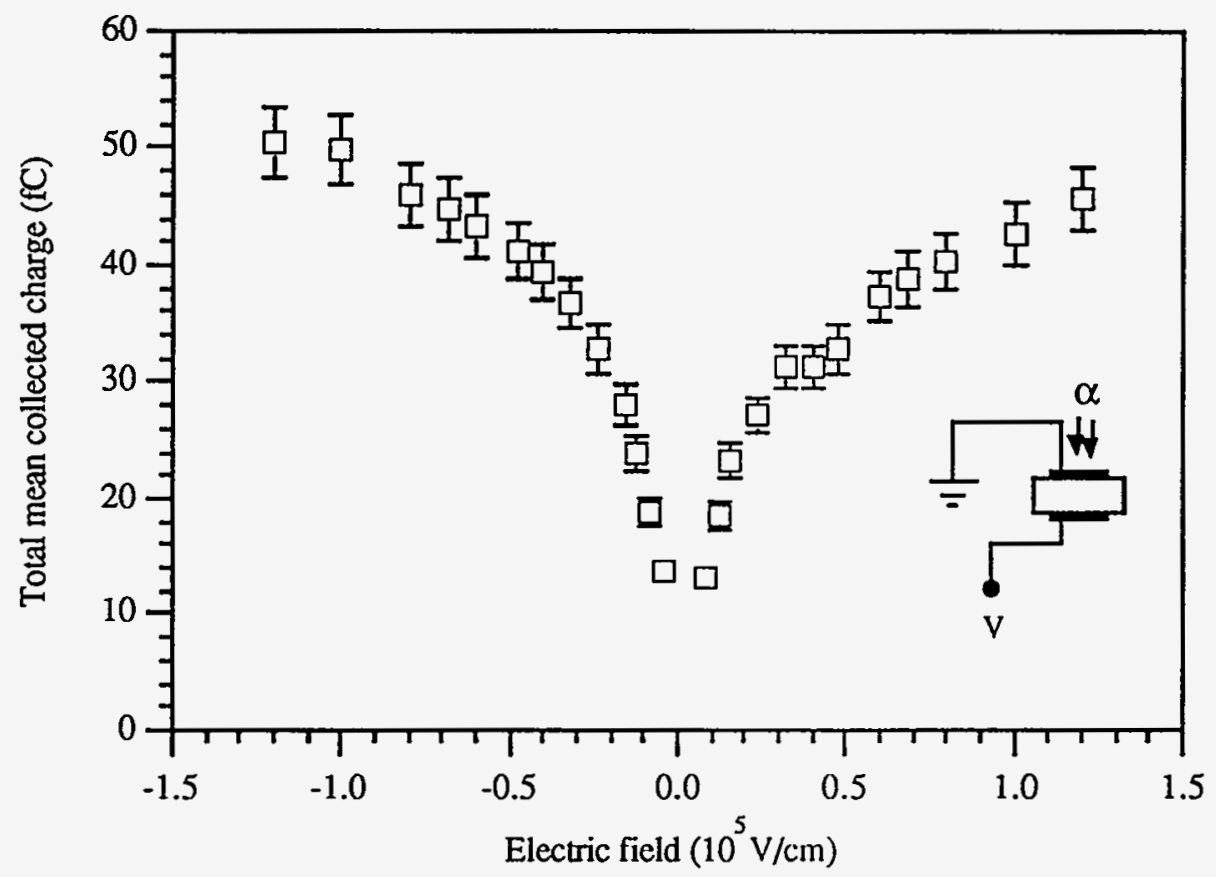

Figure 3.1.7: Total mean collected charge as a function of applied electric field for detector SYM25m. 


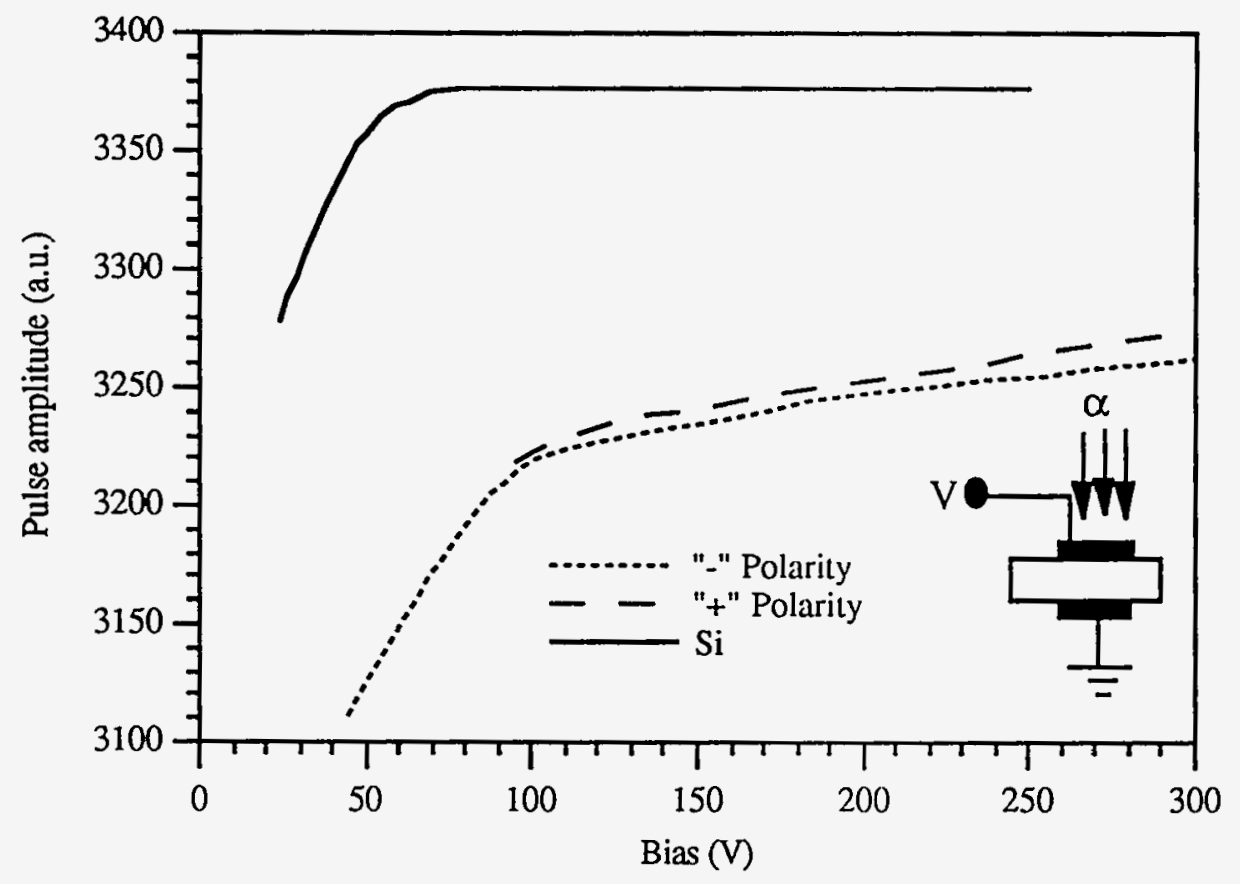

Figure 3.1.8: Mean collected charge as a function of applied bias reported by Kozlov, et al. [6]. The relative magnitude of the collected charge is defined by the pulse amplitude measured, presumably the centroid of the pulse-height distribution.

The charge collection profile for SYM70m is shown in Fig. 3.1.9 and is dominated by the asymmetry with a change in polarity. The asymmetry clearly increases as the ratio of the $\alpha$-particle range to the thickness of the detector decreases. If we compare with the extent of asymmetry seen in the data of Kozlov, et al. (Fig. 3.1.8) the asymmetry shown in Fig. 3.1.9 is much larger even though the ratio of the $\alpha$-particle range to the detector thickness is greater than for the detector used by Kozlov. This may indicate that the mean product $\mu \tau$ for the detector used by Kozlov is much larger than that of the material used for SYM70m. 


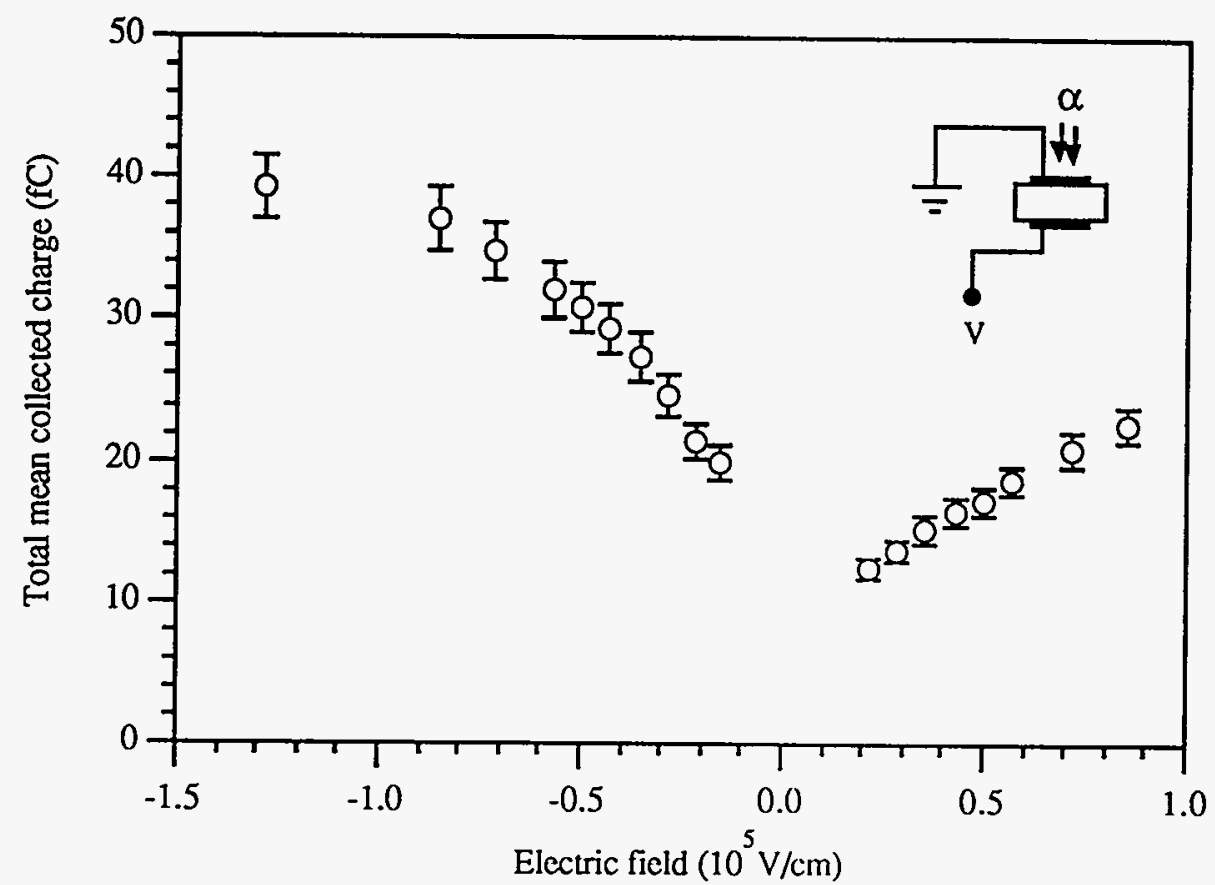

Figure 3.1.9: Total mean collected charge as a function of applied electric field for SYM70m.

The asymmetries with the three detectors studied here are compared in Fig. 3.1.10 by using the ratio of charge collected with positive polarity to charge collected with negative polarity. If the mean free paths of both electrons and holes were large compared to detector thickness, the ratios should all be unity, independent of applied electric field. Complete charge collection would always be found. For the thinnest detector, SYM14m, where the detector thickness is just slightly larger than the $\alpha$-particle range, the charge collection ratio is greater than unity at low fields $\left(<10^{4} \mathrm{~V} / \mathrm{cm}\right)$ and approaches unity asymptotically as the field increases beyond $0.5 \times 10^{5} \mathrm{~V} / \mathrm{cm}$. This behavior can be explained by assuming a shorter mean free path for electrons as compared to holes at low fields with the sum of the mean free paths for both carriers becoming large compared to $14.5 \mu \mathrm{m}$ at the highest field. For the $25 \mu \mathrm{m}$ thick detector, the charge collection ratio is well below unity at lower fields and approaches unity at the highest fields studied. For 
the thickest detector, charge collection appears incomplete at all field strengths. These results are discussed further in Sec. 3.2.

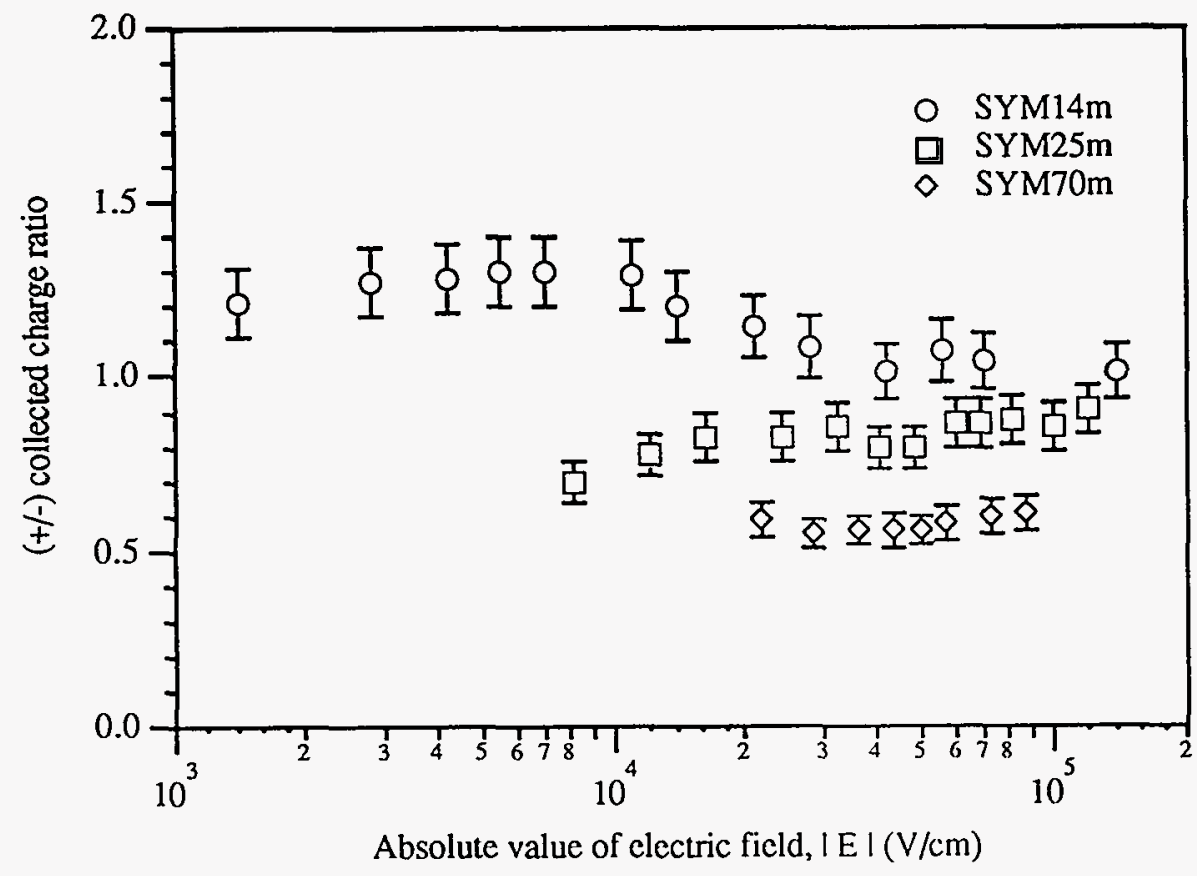

Figure 3.1.10: Ratio of total mean collected charge for positive polarity to that of negative polarity for diamond detectors varying in thickness.

\subsubsection{Detector resolution}

Measured detector resolutions are presented as the fractional resolution, DR,

$$
\text { Fractional resolution, } D R=\frac{F W H M}{\text { Mean pulse height }}
$$

In Fig. 3.1.11, the resolution for SYM14m is shown as a function of applied electric field. Similar to the profile of the collected charge, the resolution profile is nearly symmetric with respect to polarity. This seems reasonable because the excitation from $\alpha$-particles is roughly uniform between the electrical contacts. There is continuous improvement in the 


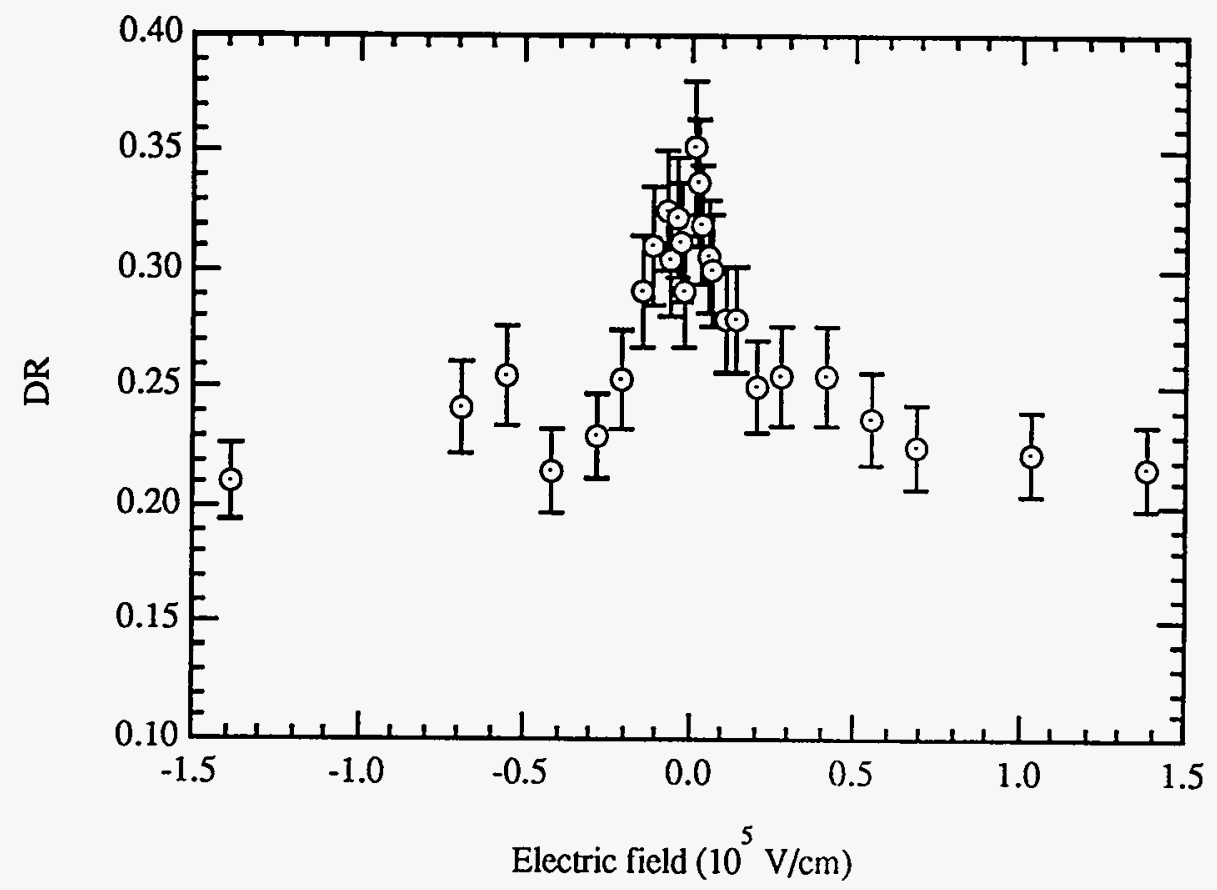

Figure 3.1.11: Fractional resolution, DR, of SYM $14 \mathrm{~m}$ as a function of applied electric field.

resolution with increasing field that approaches a value of about 0.21 at electric fields $>10^{5} \mathrm{~V} / \mathrm{cm}$. The improvement in the resolution is presumed due to the increase in collected charge.

It is important to note that the limiting resolution found experimentally is much greater than the resolution expected from production of the charge carriers. If the resolution of the detector was dependent only on the statistics of charge production, it would be expected to be approximately 0.004 (see Sec. 3.2.2). Because it has been shown that electronic noise is negligible, the only other process that can account for such poor resolution is incomplete charge collection. The effect of this is examined in subsequent sections of this chapter.

Figures 3.1.12 shows the measured resolution profile as a function of applied electric field for SYM25m. The asymmetry apparent in the profile is similar to that seen in the mean charge collection data. This supports the notion that incomplete charge 
collection is a significant factor affecting the detector resolution. The resolution at the highest field approaches about 0.17 , slightly lower than that found for SYM14m. The data for SYM70m shown in Fig. 3.1.13 suggests much the same characteristics. 


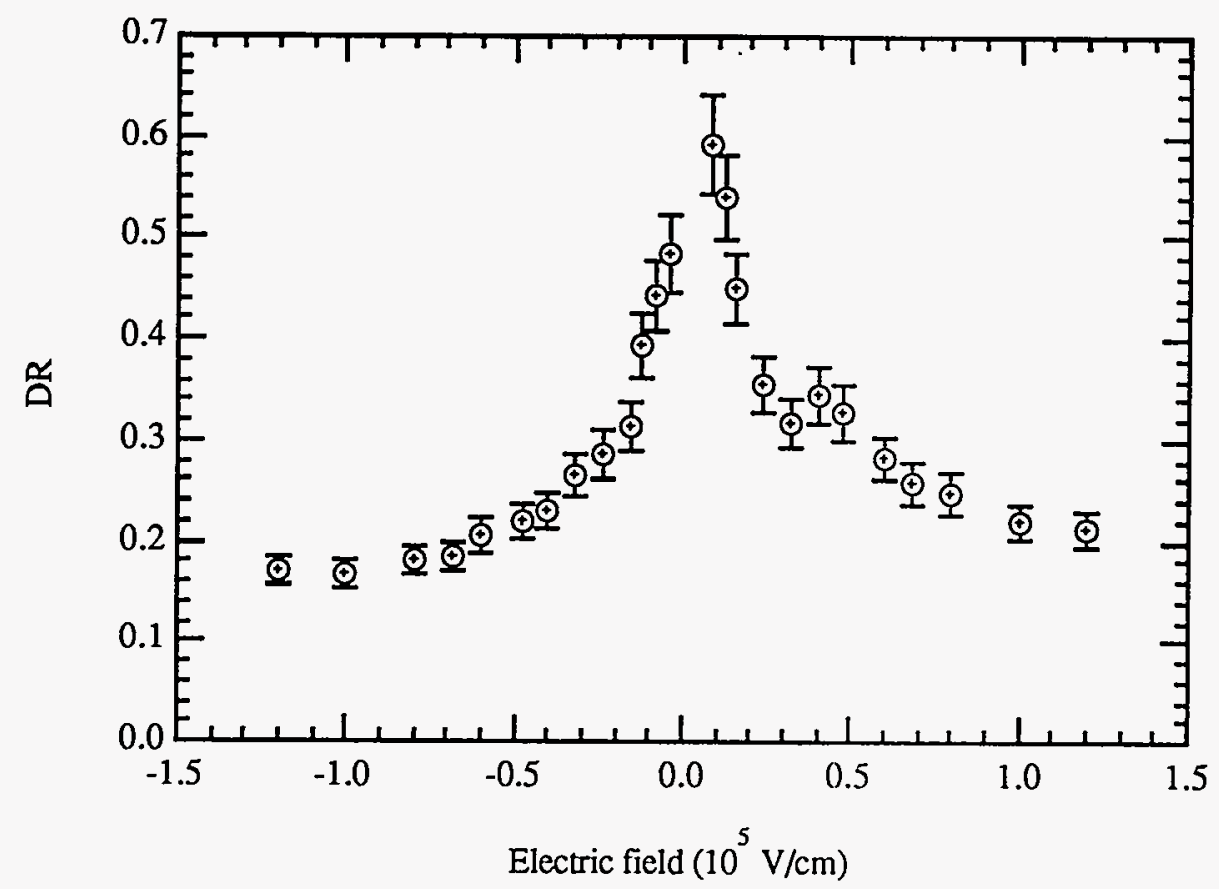

Figure 3.1.12: Fractional resolution, DR, of SYM25m as a function of applied electric field.

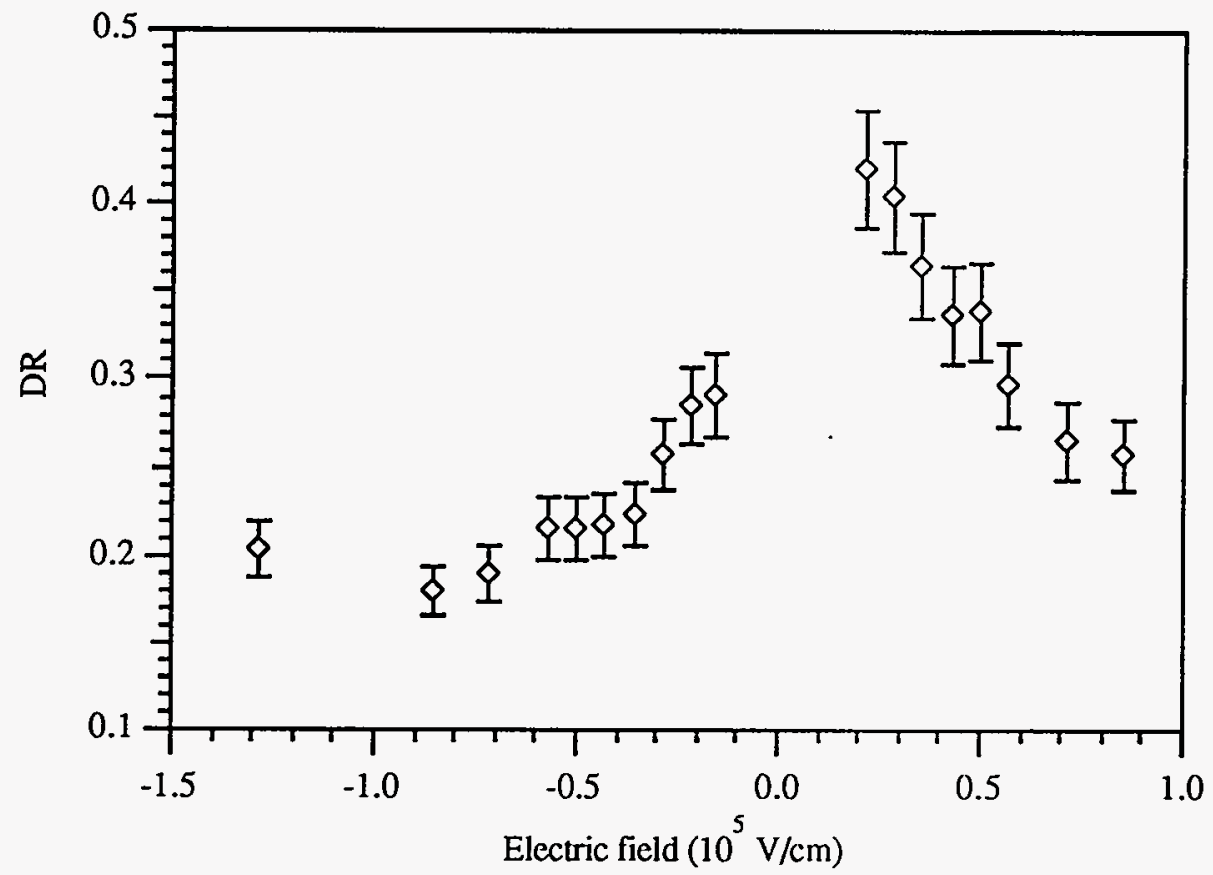

Figure 3.1.13: Fractional resolution, DR, of SYM70m as a function of applied electric field. 


\subsection{Charge collection}

\subsubsection{Model for mean collected charge}

In this section, a model for charge collection in a diamond detector is presented. The model is based on the assumption of independent transport of charge carriers and that charge is induced on the external circuit as long as the carriers are in motion. A schematic of the model is shown in Fig. 3.2.1 where $\mathrm{L}$ is the detector width (or electrical contact separation), $\mathrm{R}$ is the mean range of $\alpha$-particles that are assumed to be monoenergetic with energy $E_{o}$, and $V$ is the applied bias voltage.

From Ramo's theorem [6], the induced current due to a moving charge is given by

$$
i=\frac{d q}{d t}=\frac{Q}{L}\left(v_{e}+v_{h}\right)
$$

where $\mathrm{Q}=$ total charge in motion

$$
\begin{aligned}
v_{e} & =\mu_{\mathrm{e}}|\vec{E}|=\text { electron drift velocity } \\
\mu_{\mathrm{e}} & =\text { electron mobility } \\
v_{h} & =\mu_{\mathrm{h}}|\vec{E}|=\text { hole drift velocity } \\
\mu_{\mathrm{h}} & =\text { hole mobility } \\
\mathrm{q} & =\text { induced charge (or total collected charge in our derivation) in the } \\
& \text { external circuit due to total moving charge }
\end{aligned}
$$

The total number of carriers produced in a thickness $\mathrm{dx}$ due to ionization by an $\alpha$-particle is

$$
\begin{aligned}
& \qquad \begin{array}{l}
d N=\left(\frac{d E}{d x}\right) \frac{d x}{\varepsilon} \\
\text { where }\left(\frac{d E}{d x}\right)=\text { energy loss per unit distance (stopping power) } \\
\varepsilon=\text { energy needed to create an electron-hole pair }
\end{array}
\end{aligned}
$$




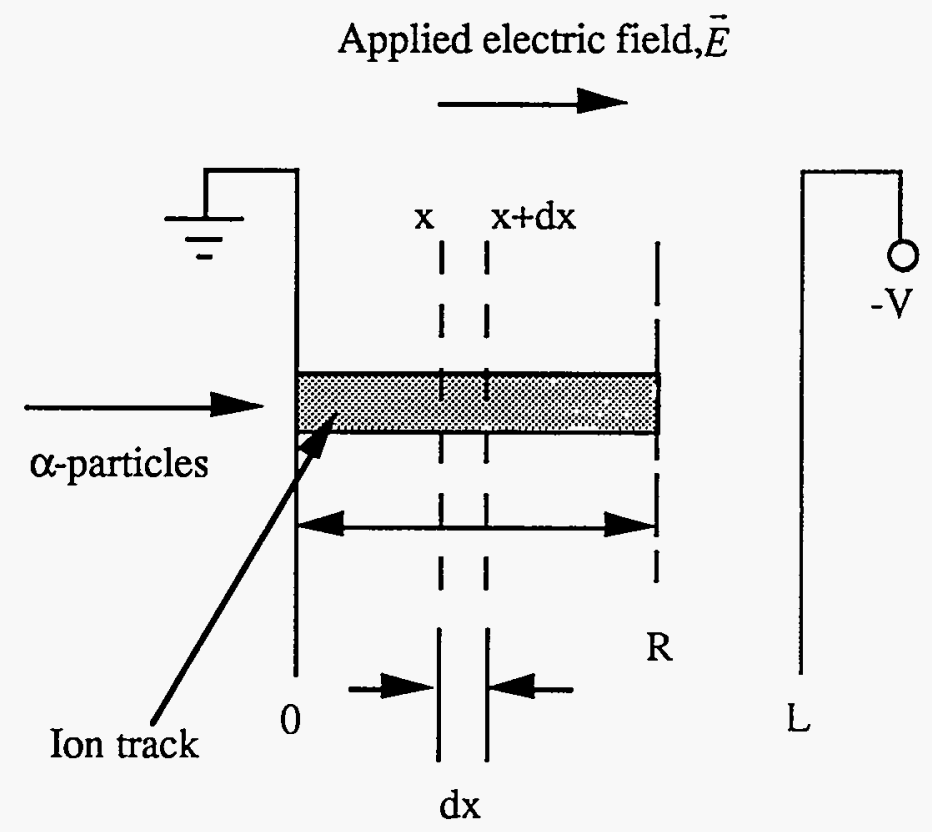

Figure 3.2.1: Schematic of model for the detectors studied. The detector is located between dimensions 0 and $L . R$ is the range of $\alpha$-particles. $\mathrm{V}$ is the applied bias and its polarity is assumed to be negative. The incident direction of the $\alpha$-particles is normal to the detector and is parallel to the applied electric field, $\vec{E} . \mathrm{x}$ is the location in the detector and $\mathrm{dx}$ is a excited slice in the detector.

By combining Eq (3.2.1), (3.2.2) and assuming that (1) the lifetimes of electrons and holes, $\tau_{\mathrm{e}}$ and $\tau_{\mathrm{h}}$, respectively, are constant, and (2) the carrier populations are assumed to be exponentially distributed in time with means characterized by their respective lifetimes, we arrive at the following expression

$$
\left(\frac{\partial^{2} q}{\partial x \partial t}\right)=\left(\frac{e}{\varepsilon L}\right)\left(\frac{d E}{d x}\right)\left(v_{e} e^{-t / \tau_{a}}+v_{h} e^{-t / \tau_{h}}\right)
$$

where $\mathrm{e}=$ elementary charge 
The assumption of constant carrier lifetimes implies constant electron and hole capture cross sections for charge carriers.

In order to perform integration over time, a maximum carrier drift time, $t_{\max }^{i}$, is chosen where i can denote either electron or hole

$$
\left(\frac{d q}{d x}\right)=\left(\frac{e}{\varepsilon L}\right)\left(\frac{d E}{d x}\right)\left[v_{e} \int_{0}^{t_{\max }^{\prime}} e^{-t / \tau_{e}} d t+v_{h} \int_{0}^{t_{\text {max }}^{h}} e^{-t / \tau_{h}} d t\right]
$$

Evaluating the integral gives

$$
\left(\frac{d q}{d x}\right)=\left(\frac{e}{\varepsilon L}\right)\left(\frac{d E}{d x}\right)\left\{\tau_{e} v_{e}\left[1-e^{-t_{\max }^{t} / \tau_{e}}\right]+\tau_{h} \nu_{h}\left[1-e^{-t_{\max }^{h} / \tau_{h}}\right]\right\}
$$

The maximum drift times for electrons and holes are defined by the types of electrical contacts, whether injecting or non-injecting. In defining the electrical contacts, it is assumed that an injecting contact will provide carriers as needed to maintain spacecharge neutrality at all times and everywhere in the detector. If any of the generated carriers is swept-out of the detector, it is assumed to be replenished by the contacts, immediately. This situation is analogous to having mean free paths of the carriers much shorter than the width of the detector such that no carrier ever reaches the detector boundaries. The maximum drift time for a carrier is infinite and is independent of where the carrier was created. If the contacts are non-injecting, a carrier is immediately lost upon reaching a contact. There then exists the possibility for space-charge build-up in some regions of the detector depending on the degree of carrier trapping, detrapping, and the magnitude of the leakage current in the detector. As explained in Chap. 1, a linear I$\mathrm{V}$ behavior (generally referred to as the requirement for having ohmic contacts) can occur by having a large surface recombination velocity at the metal/semiconductor interface. But such a large surface recombination velocity leads to non-injection of the carriers at the contacts. Therefore, the maximum drift time for these types of contacts is a function of where the carrier-pair is created, and the polarity of the contacts with respect 
to each type of carrier. The mathematical descriptions of these contacts for the maximum drift times are discussed in appendix $\mathrm{C}$ and are

$$
\begin{array}{ll}
\text { Non-injecting: } & t_{\max }^{e}=\left(\frac{x}{v_{e}}\right) \\
& \\
& t_{\max }^{h}=\left(\frac{L-x}{v_{h}}\right) \\
\text { Injecting: } & t_{\max }^{e}=\infty \\
& t_{\max }^{h}=\infty
\end{array}
$$

The limits of integration are defined by the start and the end of ionization along the $\alpha$-particle track. Therefore, the total collected charge is given by

$$
q=\left(\frac{e}{\varepsilon L}\right)\left(\int_{0}^{R}\left\{\left(\frac{d E}{d x}\right)\left\{\tau_{e} v_{e}\left[1-e^{-t_{\max }^{t} / \tau_{c}}\right]+\tau_{h} v_{h}\left[1-e^{-t_{\max }^{h} / \tau_{h}}\right]\right\}\right\} d x\right)
$$

This expression contains two independent terms; one applies to the charge collection due to electrons and the other to the charge collection due to holes, i.e.,

$$
\begin{aligned}
& q_{e}=\left(\frac{e}{\varepsilon L}\right)\left[\int_{0}^{R}\left\{\left(\frac{d E}{d x}\right)\left(\tau_{e} \nu_{e}\left[1-e^{-t_{\operatorname{tax}}^{k} / \tau_{t}}\right]\right)\right\} d x\right] \\
& q_{h}=\left(\frac{e}{\varepsilon L}\right)\left(\int_{0}^{R}\left\{\left(\frac{d E}{d x}\right)\left(\tau_{h} v_{h}\left[1-e^{-\tau_{\operatorname{mox}}^{k} / \tau_{t}}\right]\right)\right\} d x\right]
\end{aligned}
$$

and

$$
q=q_{e}+q_{h}
$$

In the limit that both contacts are injecting the exponential terms in equations 3.2.10 and 3.2.11 vanish and the integrals depend only on the total energy deposited in the detector by the incident ion. In this limit it is easily shown that 


$$
\begin{aligned}
& q_{e}=e\left(\frac{E_{o}}{\varepsilon}\right)\left(\frac{\lambda_{e}}{L}\right) \\
& q_{h}=e\left(\frac{E_{o}}{\varepsilon}\right)\left(\frac{\lambda_{h}}{L}\right)
\end{aligned}
$$

and

$$
q=q_{e}+q_{h}=\frac{e E_{o}}{\varepsilon L}\left(\lambda_{e}+\lambda_{h}\right)
$$

Here,

$$
\begin{aligned}
& \lambda_{e}=v_{e} \tau_{e} \\
& \lambda_{h}=v_{h} \tau_{h}
\end{aligned}
$$

Now, the total charge produced by ionization is

$$
Q=\frac{e E_{o}}{\varepsilon}
$$

Thus, the ratio of charge collected to the generated charge, the charge collection efficiency $\eta$ is

$$
\eta=\frac{q}{Q}=\frac{1}{L}\left(\lambda_{e}+\lambda_{h}\right)
$$

Clearly, if both of the contacts are injecting, the device can exhibit charge gain $(\eta>1)$ if

$$
\left(\lambda_{e}+\lambda_{h}\right)>L
$$

This case and that when only one contact is injecting is treated further below.

Notwithstanding the approximate nature of such calculations, it is almost always instructive to make simplifications that lead to simple analytical expressions to describe a phenomena. This leads to useful insights into the physical phenomena being modeled without undue mathematical complications. Thus, it is possible to obtain a closed form solution of Eq 3.2.10 for all possible contact types by assuming that the stopping power is constant, i.e.,

$$
\int \frac{d E}{d x} d x=R\left\langle\frac{d E}{d x}\right\rangle
$$




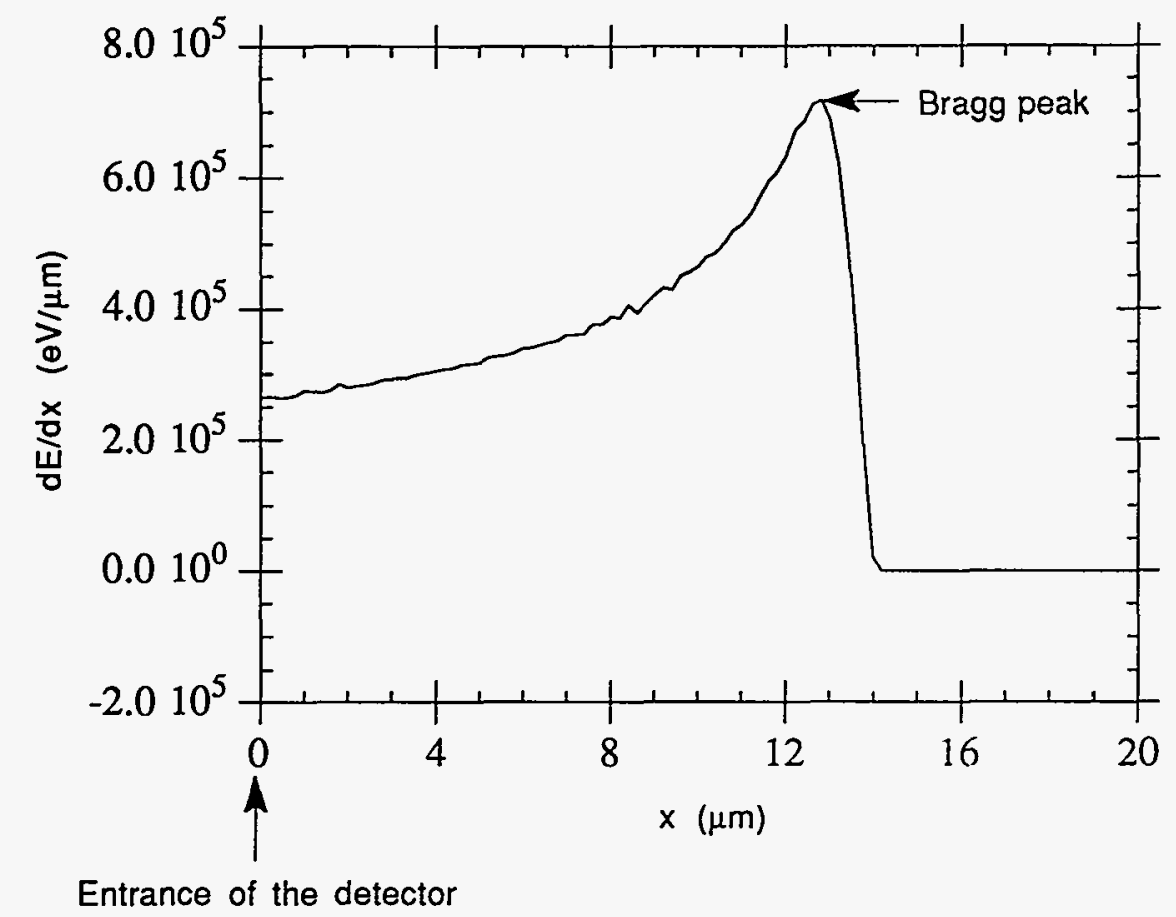

Figure 3.2.2: The TRIM-calculated stopping power distribution of 5.48-MeV $\alpha$ particle in diamond. In this calculation, diamond is assumed to be carbon with the material density of $3.51 \mathrm{~g} / \mathrm{cm}^{3}$. Any crystalline effect on the stopping power is ignored.

For 5.48-MeV $\alpha$-particles in diamond, the ratio of the stopping power at the peak of the ionization distribution to the stopping power at entrance to the detector is calculated to be less than 3 (see Fig 3.2.2). Thus, if we assume a constant stopping power, the differences in the total collected charge will not be greatly different from a calculation assuming nonconstant stopping power.

As a first example, the total collected charge when both contacts are injecting is identical to the result shown in Eq 3.2.16. For this case, the symmetry in the total collected charge is preserved regardless of the polarity of the applied field. When both contacts are non-injecting, the charge collected from electron and hole migration is given by 


$$
\begin{aligned}
& q_{h}=\left(\frac{e E_{o}}{\varepsilon}\right)\left(\frac{\lambda_{h}}{L}\right)-\left(\frac{e E_{o}}{\varepsilon}\right)\left\{\left(\frac{\lambda_{h}^{2}}{L R}\right)\left[e^{-(L-R) / \lambda_{h}}-e^{-L / \lambda_{h}}\right]\right\} \\
& q_{e}=\left(\frac{e E_{o}}{\varepsilon}\right)\left(\frac{\lambda_{e}}{L}\right)-\left(\frac{e E_{o}}{\varepsilon}\right)\left\{\left(\frac{\lambda_{e}^{2}}{L R}\right)\left[1-e^{-R / \lambda_{e}}\right]\right\}
\end{aligned}
$$

Finally, by combining appropriate expressions for the electron and hole contributions from equations $3.2 .14,3.2 .15,3.2 .21$ and 3.2.22, the charge collected from the two possible cases when one contact is injecting and the other non-injecting can be written down easily.

Each of equations 3.2.21 and 3.2.22 contains two terms. The contribution of the first to the total collected charge is linear in the number of carriers produced initially and the detector dimensions. The second term can be referred to as non-linear, and it accounts for the loss in charge due to non-injection of carrier at the contacts.

Note that the first term in each equation is identical to the charge collected when the contacts are injecting. Thus if mean free paths of both carriers are small compared to detector dimensions, the detector operation is identical to detectors equipped with injecting contacts. This is expected because if both types of carriers have such short mean free paths, they never reach the contacts and few carriers would ever interact with the detector boundaries. The injection properties of the contacts become irrelevant. The injection properties are only important if either or both of the carrier mean free paths is sufficiently large that the probability of one of them leaving the detector becomes appreciable.

From the above expressions, we can clearly see the need for large mean free paths in order to maximize charge collection when detectors are equipped with non-injecting contacts. With small mean free paths, incomplete charge collection can lead to spacecharge build-up from trapping of carriers. This will, in turn, result in further reduction of collected charge and counting efficiency due to polarization effects. 
A particular case of practical importance is when the ion range $R$, is identical to the detector thickness, $\mathrm{L}$. The total charge expected from Eq 3.2.21 and 3.2.22 then becomes

$$
q=\left(\frac{e E_{o}}{\varepsilon L}\right) \lambda-\left(\frac{e E_{o}}{\varepsilon L^{2}}\right)\left\{\lambda_{h}^{2}\left[1-e^{-L / \lambda_{h}}\right]+\lambda_{e}^{2}\left[1-e^{-L / \lambda_{c}}\right]\right\}
$$

and the nonlinear terms for both electrons and holes are identical. Therefore, charge collection symmetry as a function of bias polarity is independent of any difference in the mean free paths of the two carriers. This situation is equivalent to uniform illumination between the detector contacts, a case found in typical photoconductor applications [7]. Furthermore, Eq. 3.2.23 has been used by Trammell, et. al. |8| and McMath and Martini [9] to examine the response of thick $\gamma$-ray semiconductor detectors when the detector is uniformly irradiated.

At high fields, carrier velocities saturate. This corresponds to carrier mobilities decreasing as a function of electric field since the carrier velocity is defined as product of the carrier mobility and the electric field; carrier mobilities are approximately constant at low fields. Because we have already assumed that both lifetimes of electrons and holes are constant, and assumed that the absolute value of the applied field is constant within the detector, the only field dependence of the mean free paths is from the field-dependent mobility.

The electric field dependence of the carrier mobility, $\mu_{c}$ or $\mu_{h}$, is calculated based on an effective carrier temperature, $T_{i c}$, where $i$ can be either electrons or holes. $T_{i c}$ is calculated by assuming that the carrier kinetic energy, gained from the applied electric field, is dissipated with the emission of acoustic phonons and is given by [10]

$$
T_{i c}=\frac{T}{2}\left\{1+\sqrt{1+\frac{3 \pi}{8}\left(\frac{\mu_{o}|\vec{E}|}{c_{s}}\right)^{2}}\right\}
$$


where $T_{i c}=$ carrier temperature where $\mathrm{i}$ is electron or hole

$\mathrm{T}=$ lattice temperature

$|\vec{E}|=$ applied electric field

$c_{S}=$ speed of sound in the material

$\mu_{o i}=$ low field carrier mobility, with $\mathrm{i}$ being either $\mathrm{n}$ or $\mathrm{p}$

In the derivation, it is assumed that $|\vec{E}|$ is constant everywhere and the bias polarity is assumed to be negative. The electric field-dependent mobility can be expressed with

$$
\mu_{a i}=\sqrt{\frac{T}{T_{c i}}} \mu_{o i}
$$

where $\mu_{a i}=$ carrier mobility due solely to acoustic phonon scattering

and the carrier drift velocity, $v_{a i}$, is

$$
v_{a i}=\mu_{a i}|\vec{E}|
$$

At high enough fields, the carrier kinetic energy becomes sufficiently large to excite optical phonon modes which in turn becomes the predominant scattering mechanism. Because optical phonons are much more efficient in dissipating the free carrier energy, the carrier drift velocity saturates. Therefore, there exists a limiting carrier drift velocity that is given by

$$
v_{s}=\sqrt{\frac{8 h v_{o}}{3 \pi m_{i}^{*}}}
$$

where $h v_{o}=$ optical phonon energy, $163 \mathrm{meV}$ [11]

$$
m_{i}^{*}=\text { carrier density-of-states effective mass }
$$

In combining the drift velocities from both the acoustic and optical phonon scattering, we can utilize the Mathiesson's rule of reciprocal addition with a minor modification [12]

$$
\frac{1}{v}=\frac{(1-f)}{v_{a i}}+\frac{f}{v_{s}}
$$




$$
\text { where } f=\text { Boltzmann factor }
$$

$$
=\exp \left(-\frac{h v_{o}}{k T_{c i}}\right)
$$

This expression calculates the effective contributions of acoustic and optical phonon scattering mechanisms with the factor, $\mathrm{f}$, approaching the correct limit of 1 at large applied electric fields. The constants in the expression for the carrier velocity are expressed in terms of free electron mass, $m_{o}$, and for diamond are

$$
\begin{aligned}
& c_{s}=1.8 \times 10^{6} \mathrm{~cm} / \mathrm{sec}[13] \\
& m_{e}^{*}=0.57 m_{o}[14] \\
& m_{h}^{*}=1.2 m_{o}[15]
\end{aligned}
$$

Using the above formulation for the carrier mobilities, charge collection efficiencies corresponding to equations 3.2.21 and 3.2.22 were calculated and are shown in Fig. 3.2.3. Fig. 3.2.3-A shows the charge collection efficiency as a function of electric field with identical low-field $\mu \tau$-products for electrons and holes when both contacts are non-injecting. There is very slight asymmetry in the charge collection with bias polarity owing to the difference in the effective carrier masses that appear in the field-dependent mobility calculations. However, the asymmetry is greatly magnified in Fig. 3.2.3-B where the low-field $\mu \tau$-product for the electron is taken to be an order of magnitude smaller than that for holes. It therefore becomes clear that the major source of asymmetry in charge collection is the difference in the $\mu \tau$-products for electrons and holes for a given applied electric field strength. 


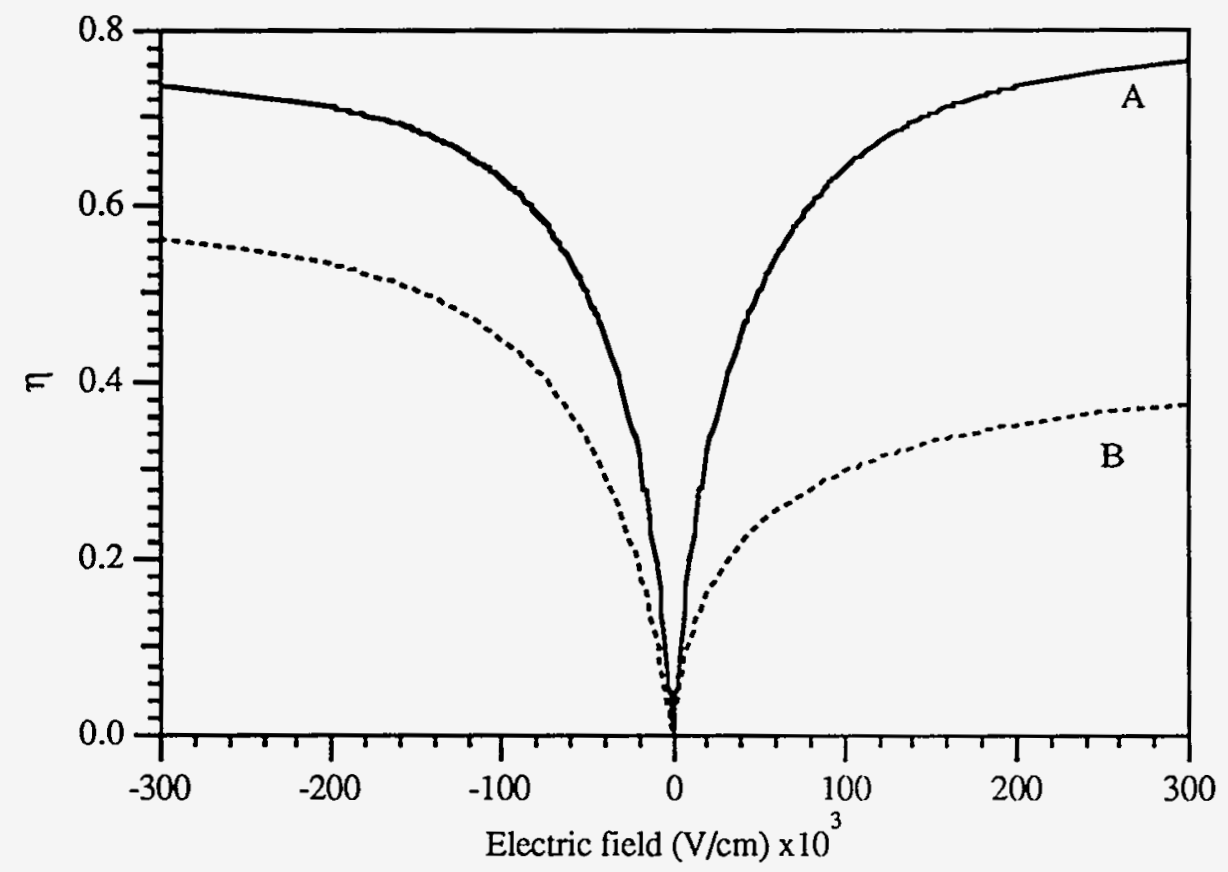

Figure 3.2.3: Charge collection efficiency, $\eta$, as a function of applied electric field where both contacts are non-injecting. The detector width is $\mathrm{L}=14.5 \mu \mathrm{m}$. (A) Charge collection efficiency when the low field $\mu \tau$-products for electrons and holes are the identical and equal to $3 \times 10^{-8} \mathrm{~cm}^{2} / \mathrm{V}$. (B) Charge collection efficiency when the lowfield $\mu \tau$-product for electrons is an order of magnitude less than that for holes.

Charge collection efficiencies clearly depend upon both the mean free paths of the carriers and the ratio of the ion range to detector thickness. This is illustrated in Fig. 3.2.4 for a range of parameters that are relevant in the detector systems studied here, assuming both electrodes are of the non-injecting type. The difference between electron and hole collection efficiencies is greatest for the smallest (R/L) ratio. As $R / L$ approaches 1, the two collection efficiencies become identical (See Equ. 3.2.22). The variation of electron and hole collection efficiencies with $\mathrm{R} / \mathrm{L}$ is the basis for time-of- 


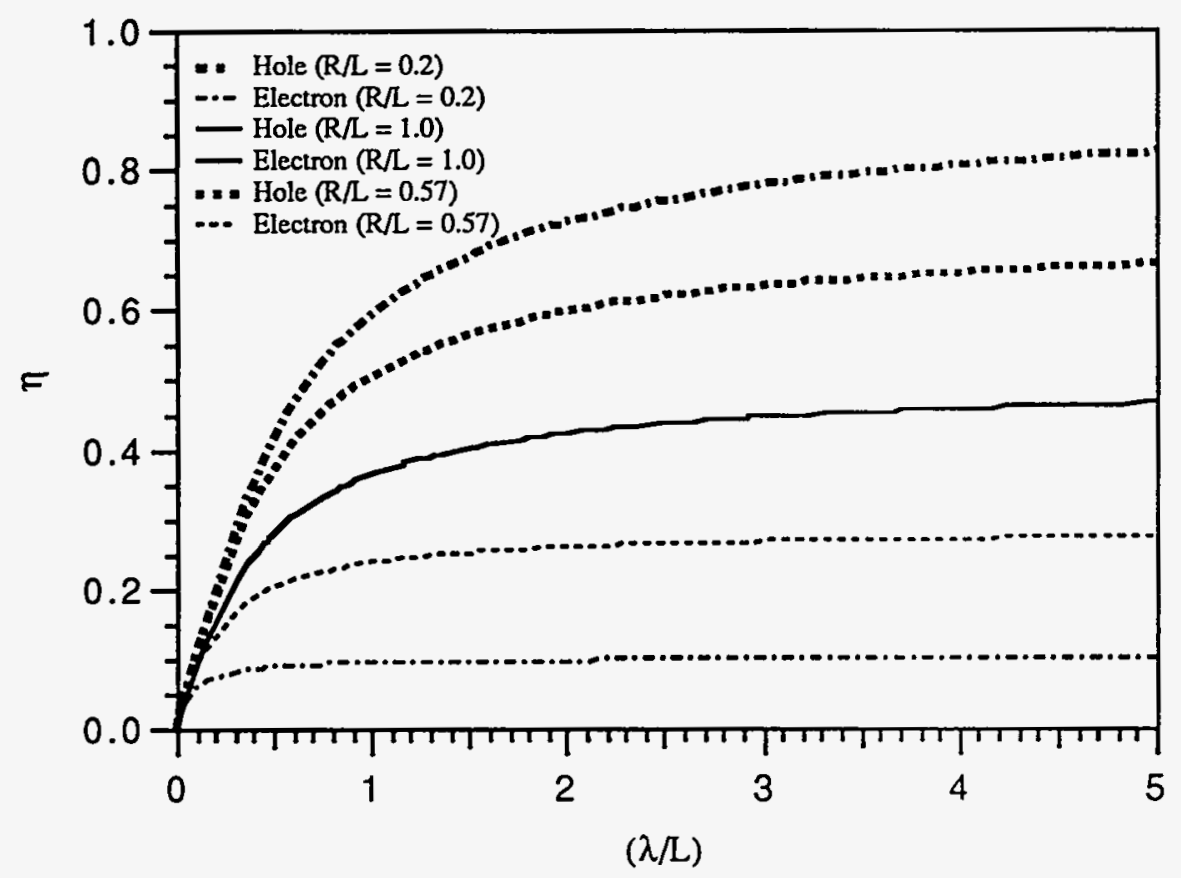

Figure 3.2.4: Illustrations of charge collection efficiencies for two non-injecting contacts as a function of the ratio of carrier mean free path to detector width (electrode separation) $\lambda / L$ and the ratio of the mean ion range to detector width. The material parameters used are $\mu_{\mathrm{oe}}$ $=200 \mathrm{~cm}^{2} / \mathrm{V}$-sec, $\mu_{\mathrm{oh}}=148 \mathrm{~cm}^{2} / \mathrm{V}$-sec, $\tau_{\mathrm{c}}=150 \mathrm{ps}, \tau_{\mathrm{h}}=608 \mathrm{ps}$.

flight experiments that measure carrier transport properties using non-penetrating radiation [16].

By use equations 3.2.14, 3.2.15, 3.2.21, and 3.2.22, the charge collection efficiency profile was calculated as a function of electric field and character of the electrical contacts. The material and operational parameters used in the calculations are

$$
\begin{gathered}
\mu_{\mathrm{oe}}=200 \mathrm{~cm}^{2} / \mathrm{V}-\mathrm{sec}, \mu_{0 h}=148 \mathrm{~cm}^{2} / \mathrm{V}-\mathrm{sec} \\
\tau_{\mathrm{e}}=150 \mathrm{ps}, \tau_{\mathrm{h}}=608 \mathrm{ps} \\
\text { detector thickness, } \mathrm{L}=25 \mu \mathrm{m}
\end{gathered}
$$




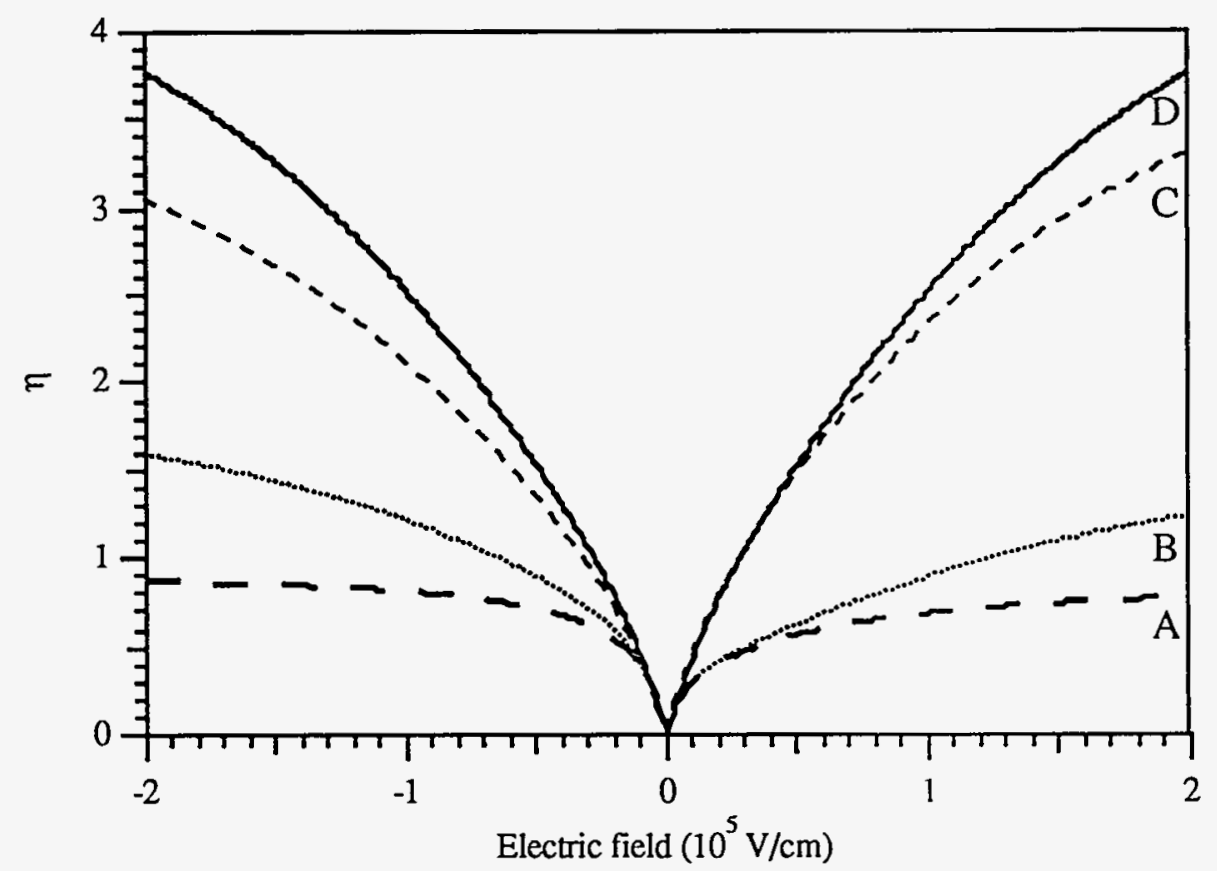

Figure 3.2.5: Charge collection efficiency, $\eta$, as a function of electrical contacts using constant stopping power. The operational and material parameters used to calculate the distributions are: $\mu_{\mathrm{oc}}=200$ $\mathrm{cm}^{2} / \mathrm{V}-\mathrm{sec}, \mu_{\mathrm{oh}}=148 \mathrm{~cm}^{2} / \mathrm{V}-\mathrm{sec}, \tau_{\mathrm{c}}=150 \mathrm{ps}, \tau_{\mathrm{h}}=608 \mathrm{ps}, \mathrm{L}=25 \mu \mathrm{m}$. A) Two non-injecting contacts. B) Electron non-injecting and hole injecting contacts. C) Electron injecting and hole non-injecting contacts. D) Two injecting contacts.

These parameters were chosen for illustrative purposes and they are representative of 25 $\mu \mathrm{m}$ thick detector studied. As seen in Fig. 3.2.5, if either or both of the contacts is injecting, there exists a possibility of gain $(\eta>1)$. If both contacts are non-injecting, however, the charge collection efficiency can never exceed unity. Clearly, the greatest charge collection efficiency is found when both contacts are injecting, whereas the smallest charge collection efficiency is found when both contacts are non-injecting. This is due to the fact that with injecting contacts, the carriers contribute linearly 
$(\operatorname{as}(\mu \tau|\vec{E}| / L))$ to the collected charge whereas in the non-injecting system, the exponential term in $(\mu \tau|\vec{E}| / L)$ is subtracted from the linear term. In comparing experimental results to the model, all four contact combinations are considered to deduce the most likely behavior of the electrical contacts used in our detectors.

A calculation identical to the one discussed above was performed except for use of a realistic stopping power distribution calculated with the code TRIM and the resulting $\eta$ are shown in Fig. 3.2.6. The profiles are very similar to those shown in Fig. 3.2.5. To examine the difference between the two stopping power assumptions, we have calculated the relative difference in efficiencies defined as

$$
\text { Relative difference }=\frac{\eta_{T R I M}-\eta_{\text {const }}}{\eta_{\text {TRIM }}}
$$

Fig. 3.2.7 shows the relative differences for the four possible contact systems. When both contacts are injecting, the relative difference is expected to be zero because charge collection is due entirely to the number of carrier-pairs initially created and their motion. The important point is that the largest relative difference is only about 0.05 and occurs at the intermediate electric fields. 


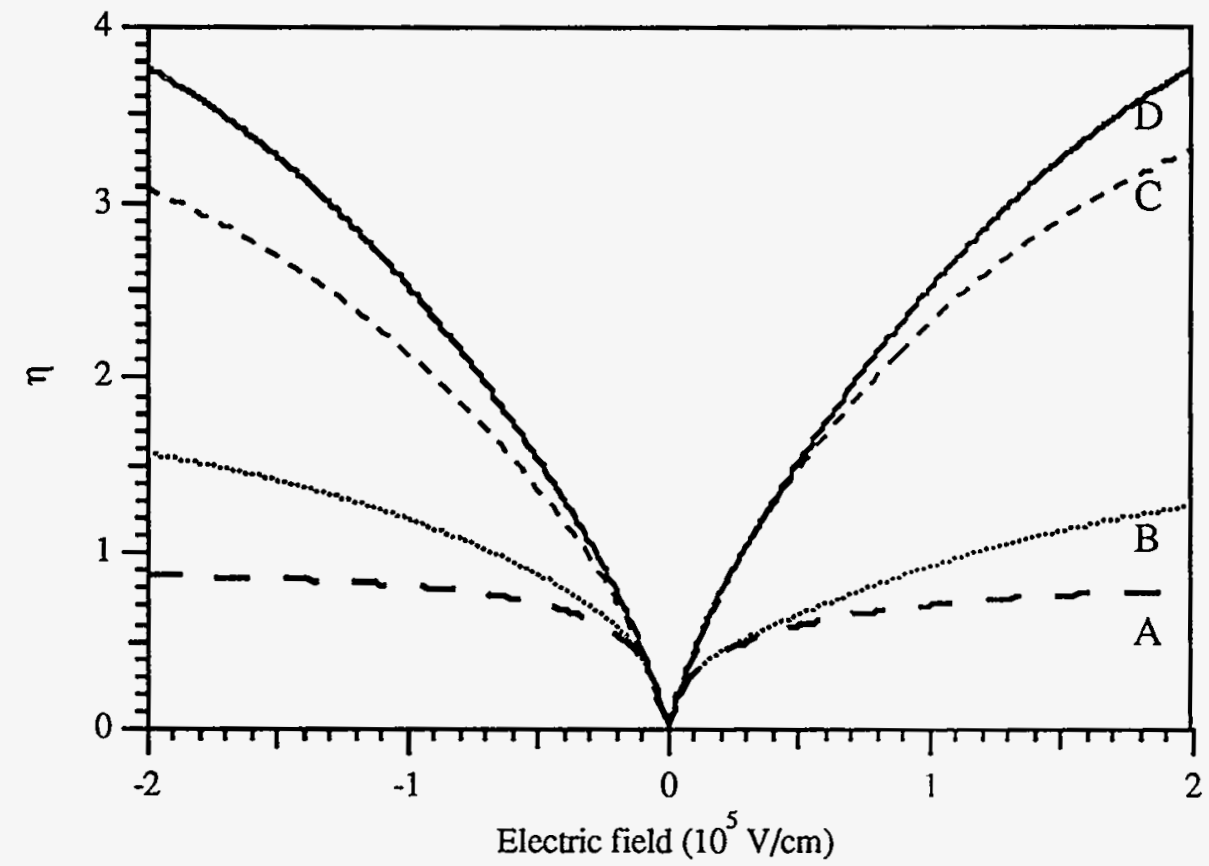

Figure 3.2.6: Charge collection efficiency, $\eta$, as a function of electrical contacts using TRIM-calculated stopping power. The operational and material parameters used to calculate the distributions are: $\mu_{0 e}=$ $200 \mathrm{~cm}^{2} / \mathrm{V}$-sec, $\mu_{\mathrm{oh}}=148 \mathrm{~cm}^{2} / \mathrm{V}$-sec, $\tau_{\mathrm{c}}=150 \mathrm{ps}, \tau_{\mathrm{h}}=608 \mathrm{ps}, \mathrm{L}=25$ $\mu \mathrm{m}$. A) Two non-injecting contacts. B) Electron non-injecting and hole injecting contacts. C) Electron injecting and hole noninjecting contacts. D) Two injecting contacts. 


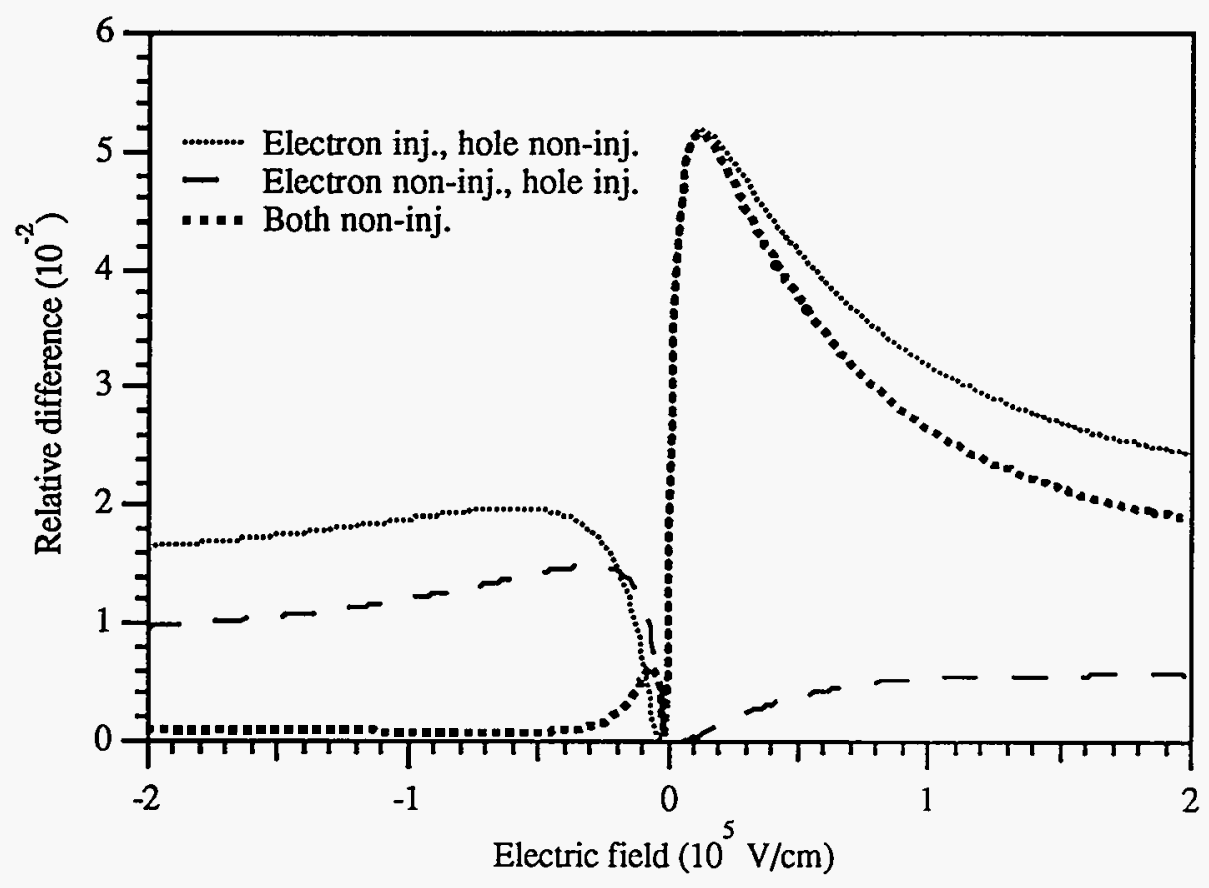

Figure 3.2.7: Comparison between calculated charge collection efficiencies with the constant stopping power assumption and the stopping power calculated with TRIM.

3.2,2 Comparison between calculated and experimental collected charge

Comparisons between data taken with the three diamond detectors and the general model as expressed by equations 3.2.11 - 3.2.13 were performed with two purposes in mind. First, the general issue of whether the model is an adecjuate representation of the detectors could be approached by determining simply if the equation led to high-quality fits to experimental data, and the parameters derived from the fits were physically reasonable. Second, if high-quality fits were obtained, then the issue of whether the simple spectrometric measurements performed here represented a means for determination of electrical properties of semiconductor detectors, or at least a good screening method for comparison of different materials, could be determined.

Fits with the present model involve essentially six free parameters; electron mobility and lifetime, hole mobility and lifetime, energy required to produce an electron- 
hole pair, and specification of the characteristics of the electrical contacts. Given the limited statistical quality of the data obtained, it is reasonable to expect to see if model fits are adequate, but somewhat optimistic to expect to obtain fitting parameters with small uncertainties. Therefore, an alternative approach was used to try and reduce the number of free parameters in all fits.

Although the magnitudes of low-field electron and hole mobilities reported in the literature have substantial scatter, this is not true for the ratios of mobilities reported by various workers. In Table 3.2.1, individual values for mobilities and their ratios are shown. The average value of the mobility ratio of electron mobility to hole from the measurements included in the table is $1.34 \pm 0.2$. As a result, this ratio was held fixed at 1.34 for most calculations.

Second, the contacts used on all three detectors were essentially identical, and there is no reason to believe that materials properties varied so much that their injecting characteristics should vary among the devices. As a result, once the nature of the contacts was inferred from one detector, they were assumed to be known for all.

Table 3.2.1: List of electron and hole mobilities reported by other investigators at $300 \mathrm{~K}$. The mean and the standard deviation of the ratio is $1.34 \pm 0.2$

\begin{tabular}{|cccc|}
\hline Source & Electron mobility, $\mu_{\mathrm{e}}$ & Hole mobility, $\mu_{\mathrm{h}}$ & Ratio, $\frac{\mu_{e}}{\mu_{h}}$ \\
\hline Pan [9] & 2000 & 1200 & 1.68 \\
Redfield [17] & 1500 & 1200 & 1.25 \\
Konorova, et al. [18] & 2000 & 1500 & 1.33 \\
Chuenkov [19] & & 2000 & \\
Kozlov, et al. [20] & 2000 & 1550 & 1.29 \\
Canali, et al. [21] & 2400 & 2100 & 1.14 \\
\hline
\end{tabular}


Third, and again because the materials properties of the three detectors is not expected to vary greatly, the energy required to produce an ion pair by alpha bombardment should not vary significantly from detector to detector. Once measured on one device, it can be considered known.

With this approach, the fits with the model involved three free parameters. In effect they provide the two mean carrier lifetimes and one carrier mobility that are selfconsistent with the mobility ratio, contact characteristics, and energy required to produce an ion pair that were assumed. Estimates of how much bias is produced with this approach can be obtained by comparison of the results for this procedure with those obtained when all six parameters were free.

Figures 3.2.8-3.2.11 show fits of the model to the experimental data from SYM25m with different contact systems. All of the remaining five parameters were considered free. From inspection of the model equation, we expect symmetry between data taken with the opposite polarities with two injecting contacts. The experimental data clearly display significant asymmetry. As seen in Fig. 3.2.8, this assumption does not reproduce the data at all. Figures 3.2.9 and 3.2.10 show model fits when one contact was injecting and the other non-injecting. Both show asymmetry with change in polarity and reproduce the experimental data well except as high fields are approached. In Fig. 3.2.11, the fit when both contacts are non-injecting is shown and clearly this is the highest quality of all fits. From these comparisons, we can conclude that the two electrical contacts are most likely not injecting to both electrons and holes. However, it cannot be completely ruled out that the contacts have some small injecting character.

In attempts to fit the model to data taken with detectors SYM14m and SYM70m, the value for $\varepsilon$ obtained with detector SYM $25 \mathrm{~m}$ was used and the contacts were assumed to be non-injecting. The value of $\varepsilon$ was found to be $14.1 \pm 0.5 \mathrm{eV}$. The number of free fitting parameters was reduced to three which are $\mu_{\mathrm{e}}, \tau_{\mathrm{e}}$ and $\tau_{\mathrm{h}}$, and this, in principle, 


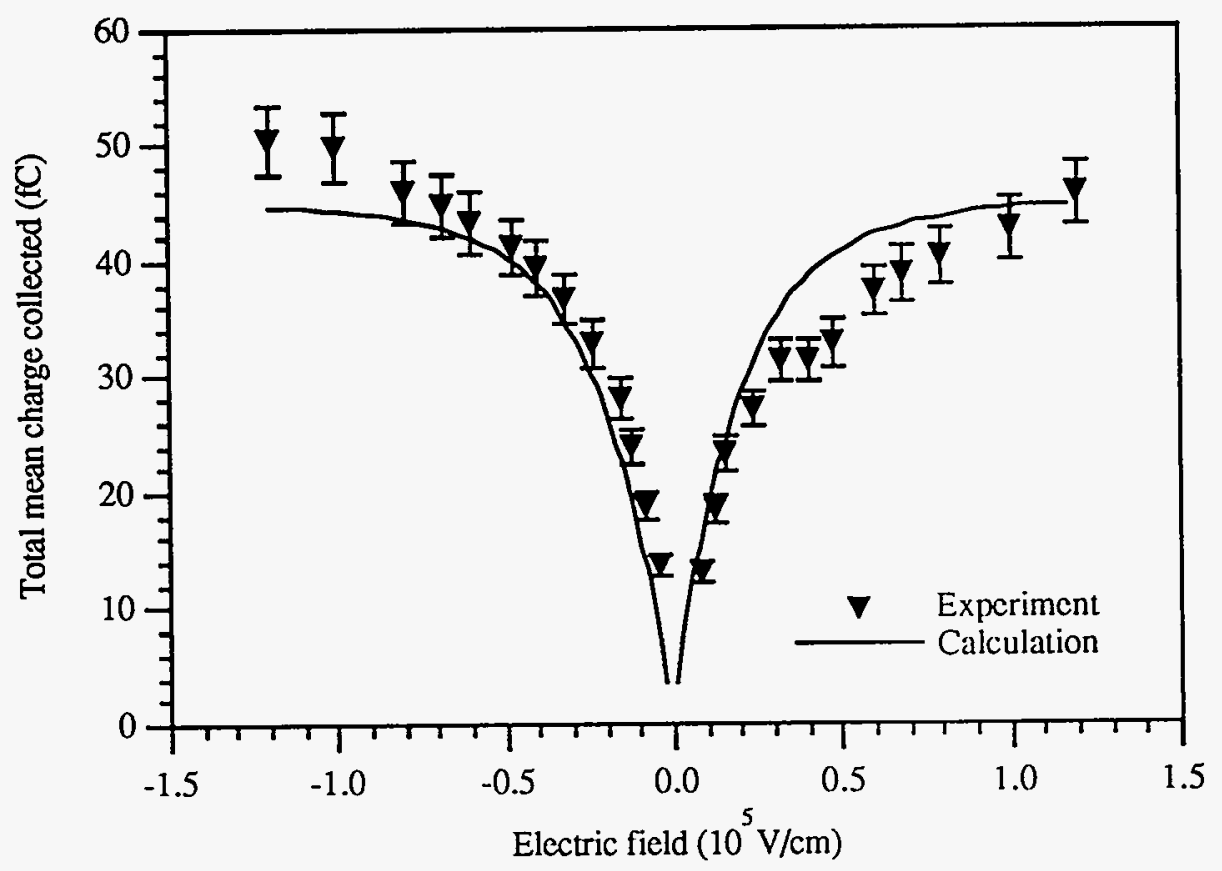

Figure 3.2.8: Comparison between the model and the experimental data for SYM25m assuming both contacts to be injecting.

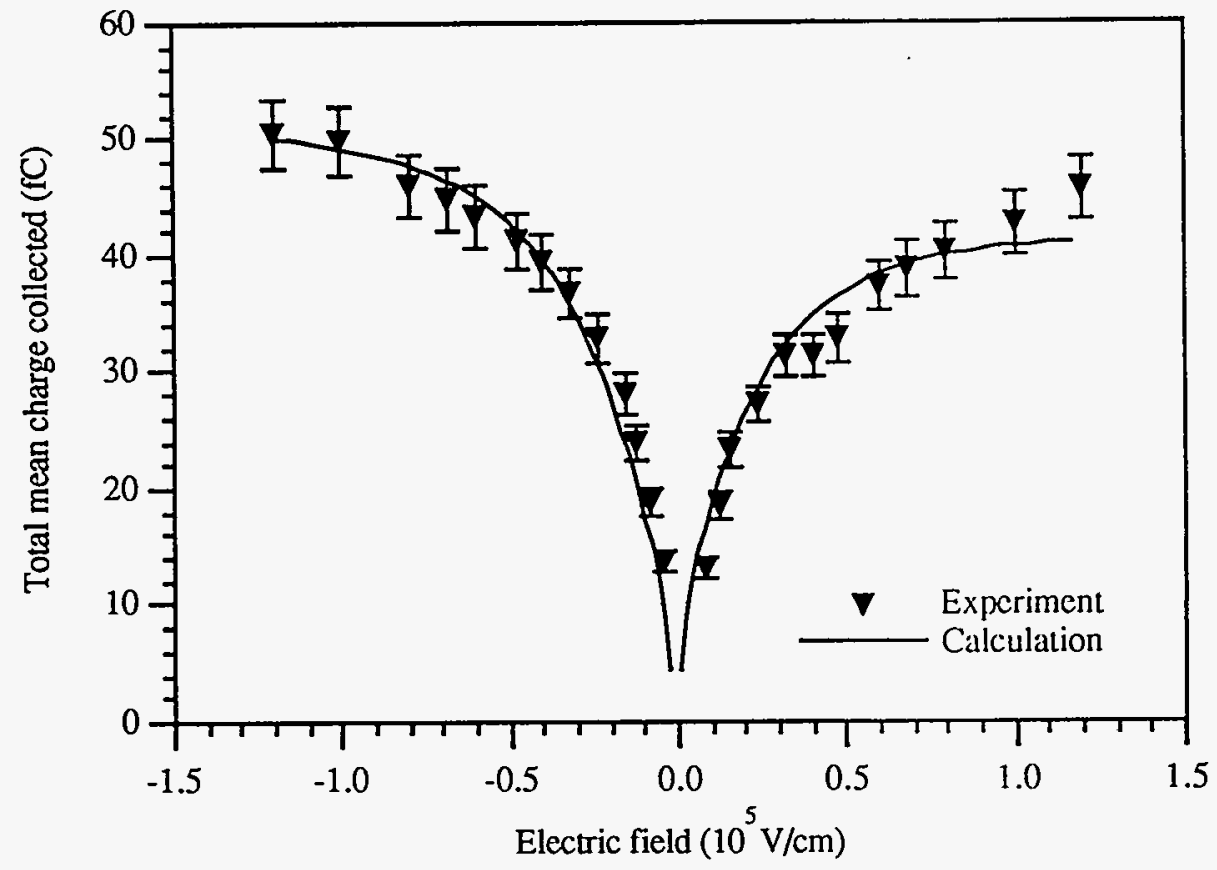

Figure 3.2.9: Comparison between the model and the experimental data for SYM25m assuming one contact to be electron injecting and the other non-injecting to holes. 


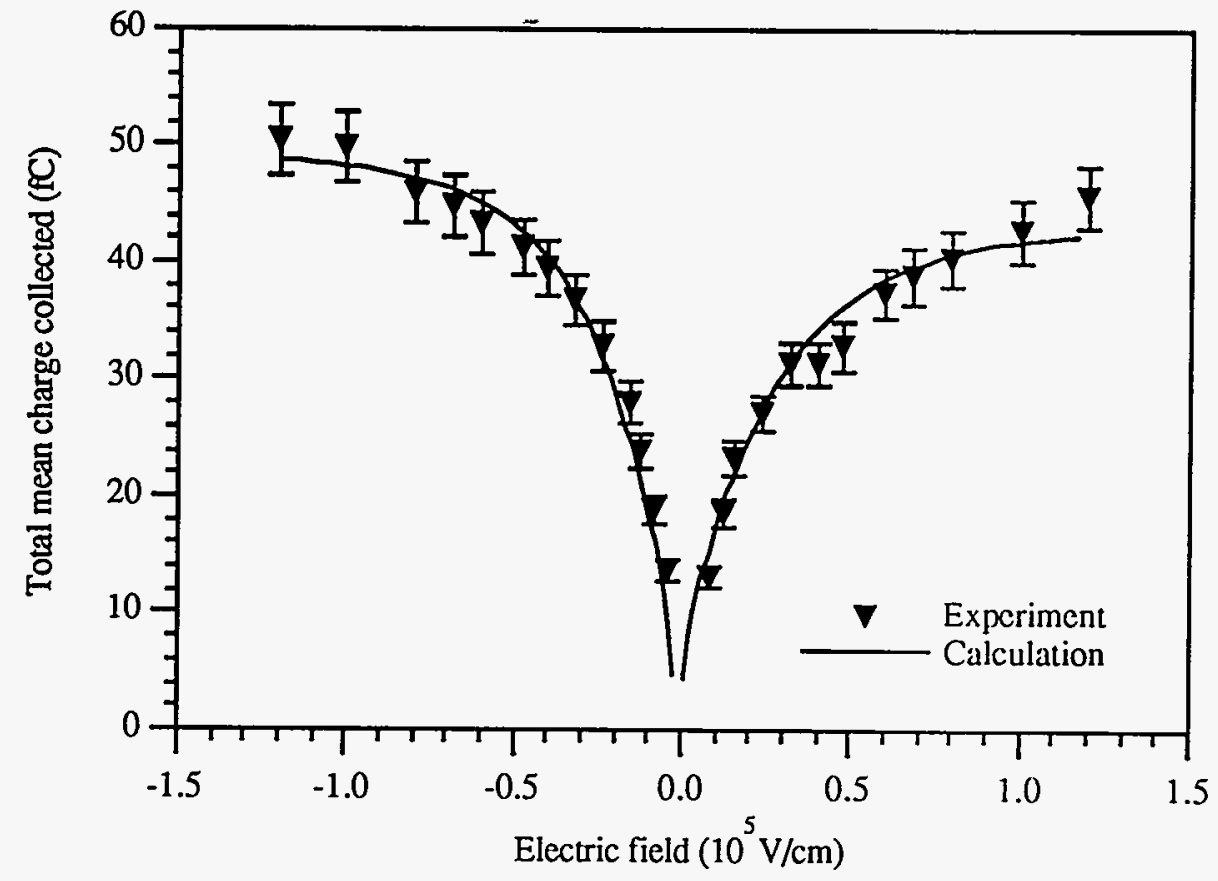

Figure 3.2.10: Comparison between the model and the experimental data for SYM25m assuming one contact to be hole injecting and the other non-injecting to electrons.

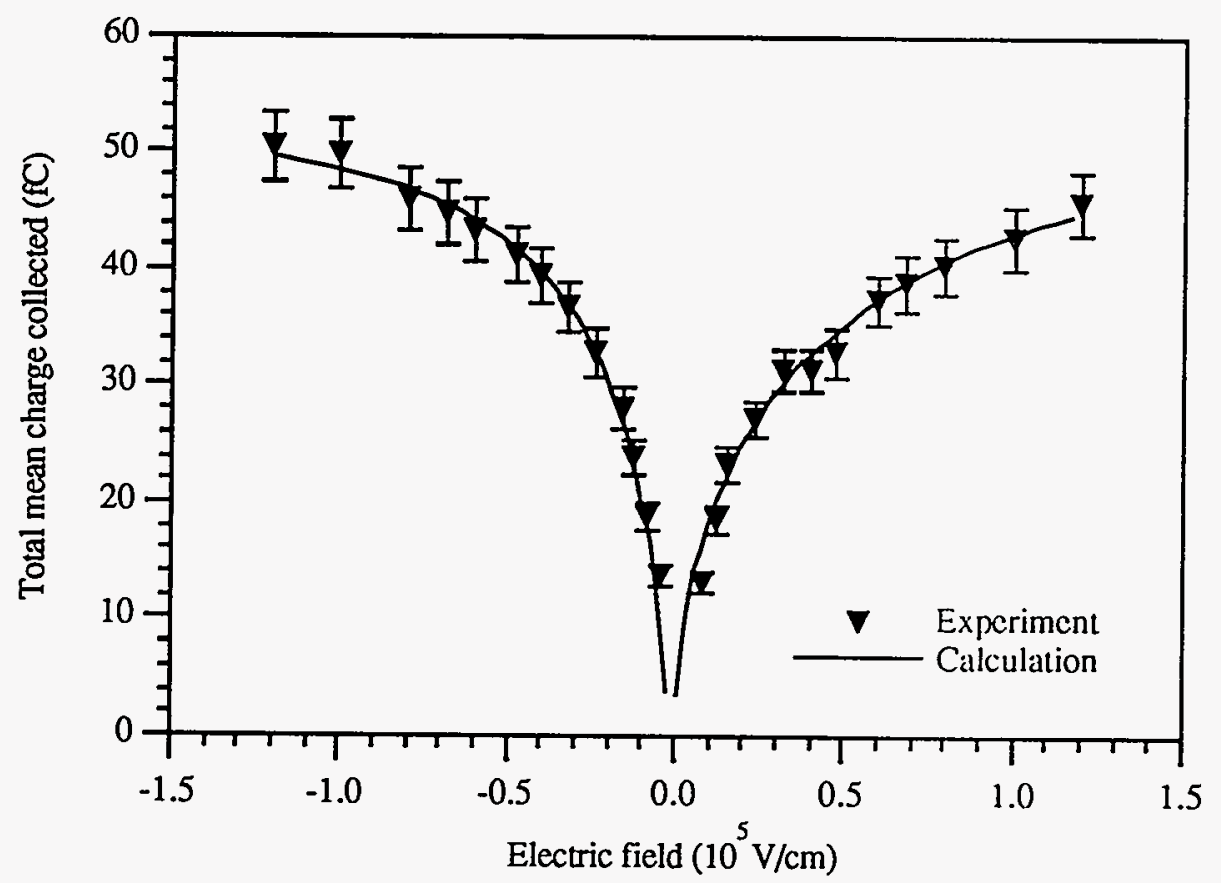

Figure 3.2.11: Comparison between the model and the experimental data for SYM25m using non-injecting contacts for the both contacts. 
should reduce the uncertainties for these parameters. The fits to the data are shown in Fig. 3.2.12 and Fig 3.2.13 and are of comparable quality to that seen for detector SYM25m. The parameters derived from these fits along with the estimated errors $( \pm \sigma)$ are shown in Table 3.2.1.

The effect of allowing $\varepsilon$ to vary was examined by fitting the model to the experimental data for SYM14m and SYM70m. The carrier mobility ratio was held constant at 1.34. The calculations are compared to the experimental data in figures 3.2 .14 and 3.2.15. The fits are again of high quality and virtually identical to those shown in figures 3.2.12 and 3.2.13. The parameters obtained from these fits are listed in Table 3.2.2. The estimated errors on parameters for SYM14m and SYM70m are much larger in comparison to those obtained with fixed $\varepsilon$. When compared with the results given in Table 3.2.2, the carrier parameters agree within $1 \sigma$ for SYM $14 \mathrm{~m}$ and $1.4 \sigma$ for $S Y M 70 \mathrm{~m}$, but a significant change in $\varepsilon$ is seen for SYM70m. The significant change in $\varepsilon$ for SYM70m is likely due to the lack of the low-field data in comparison to the other two detectors.

As another check, we performed model fits when all five material parameters were free assuming contacts were non-injecting. The parameters found are shown in Table 3.2.3. Some of the errors are larger than the mean values themselves and it is clear that the data quality of model assumptions do not permit "unique" fits to be obtained. However, it is interesting to note that $\varepsilon$ does not vary greatly. This is reasonable because $\varepsilon$ affects only the absolute magnitudes of the collected charge and the shapes of the profiles are determined by low-field $\mu \tau$-products. We can expect that $\varepsilon$ will be sensitive to the estimated mean values of the low-field $\mu \tau$-products and this is reflected in the data of Table 3.2.3.

In addition, literature values for $\varepsilon$ are listed in Table 3.2.4. The values of $\varepsilon$ from all three detectors from Table 3.2.3 are compared to the ones shown in Table 3.2.6, they all lie within the range of the reported values. The deduced parameters appears to be 


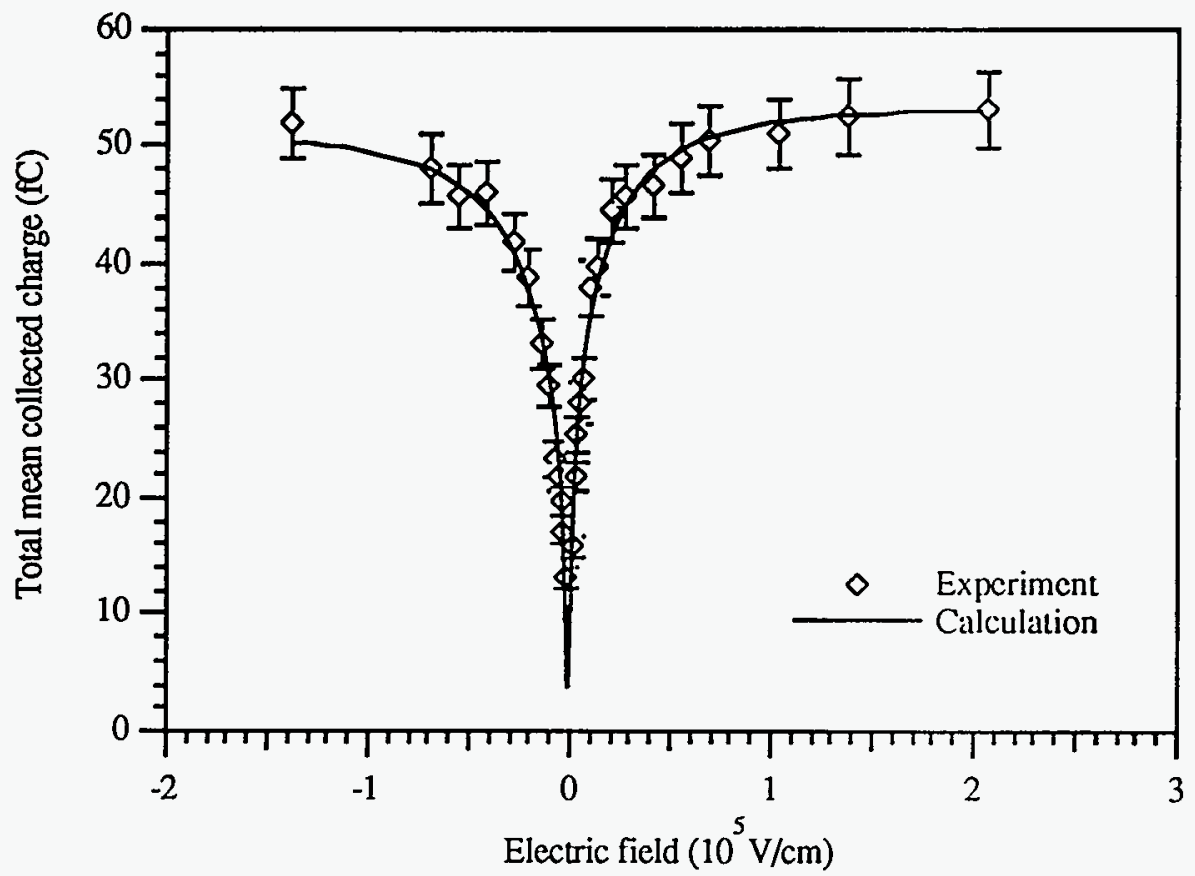

Figure 3.2.12: Comparison between the experiment and the model for SYM14m.

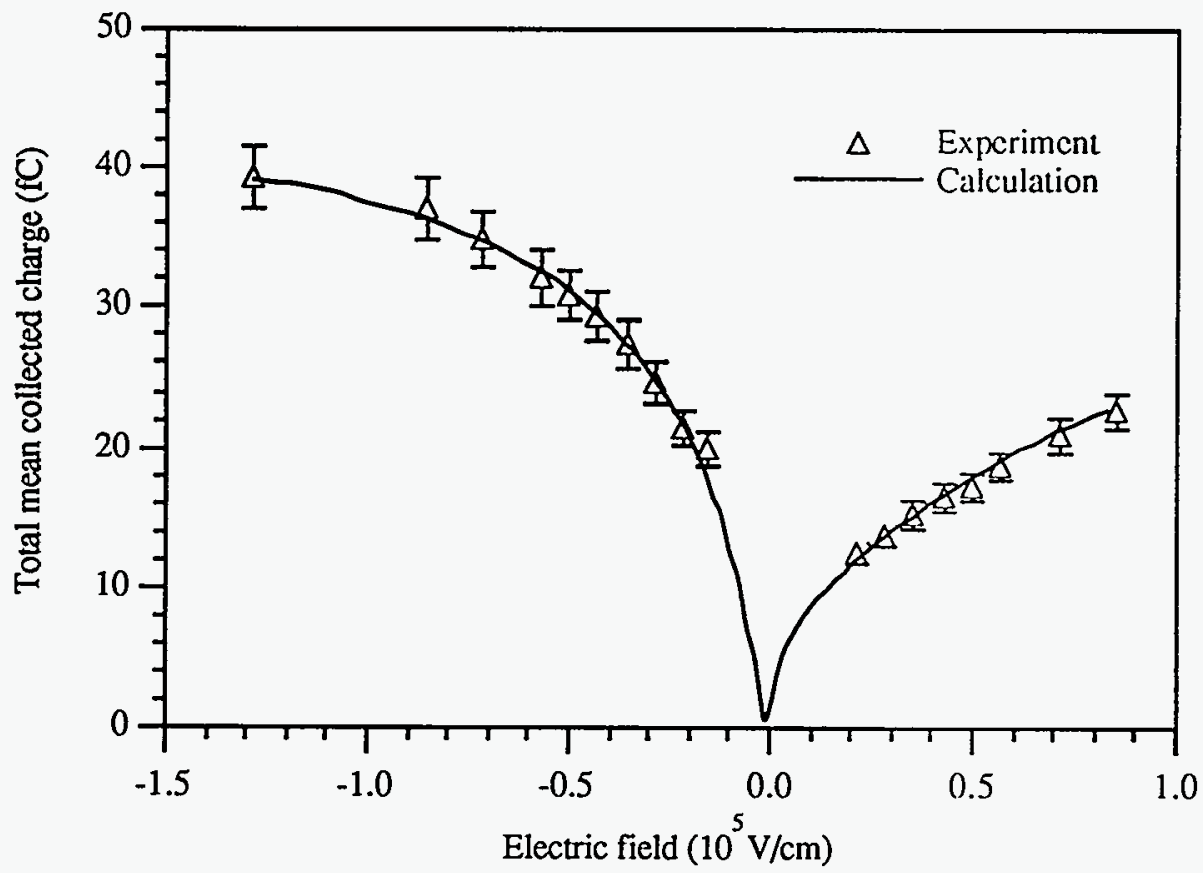

Figure 3.2.13: Comparison between the experiment and the model for SYM70m. 
Table 3.2.1: Estimated material parameters obtained from fitting the model to the experimental data for all detectors studied. The errors on the estimated parameters are just the estimated errors from non-linear least squares fitting. $\varepsilon$ was fixed at $14.1( \pm 0.5) \mathrm{eV}$ and the ratio of electron mobility to that of holes was taken to be 1.34. The contacts were assumed to be noninjecting. $\varepsilon$ was obtained by fitting the experimental data from SYM25m with the model.

\begin{tabular}{|cccccc|}
\hline Detector & $\begin{array}{c}\mu_{\mathrm{e}} \\
\left(\mathrm{cm}^{2} / \mathrm{V}-\mathrm{sec}\right)\end{array}$ & $\begin{array}{c}\mu_{\mathrm{h}} \\
\left(\mathrm{cm}^{2} / \mathrm{V}-\mathrm{sec}\right)\end{array}$ & $\begin{array}{c}\tau_{\mathrm{c}} \\
(\mathrm{ps})\end{array}$ & $\begin{array}{c}\tau_{\mathrm{h}} \\
(\mathrm{ps})\end{array}$ \\
\hline SYM14m & $571.1 \pm 135.0$ & $426.2 \pm 100.7$ & $63.8 \pm 8.1$ & $503 \pm 138.0$ \\
SYM25m & $244.6 \pm 124.0$ & $182.5 \pm 92.5$ & $124.6 \pm 55.9$ & $433.0 \pm 203.0$ \\
SYM70m & $480.3 \pm 45.6$ & $358.4 \pm 34.0$ & $111.4 \pm 7.4$ & $418.3 \pm 24.5$ \\
\hline
\end{tabular}




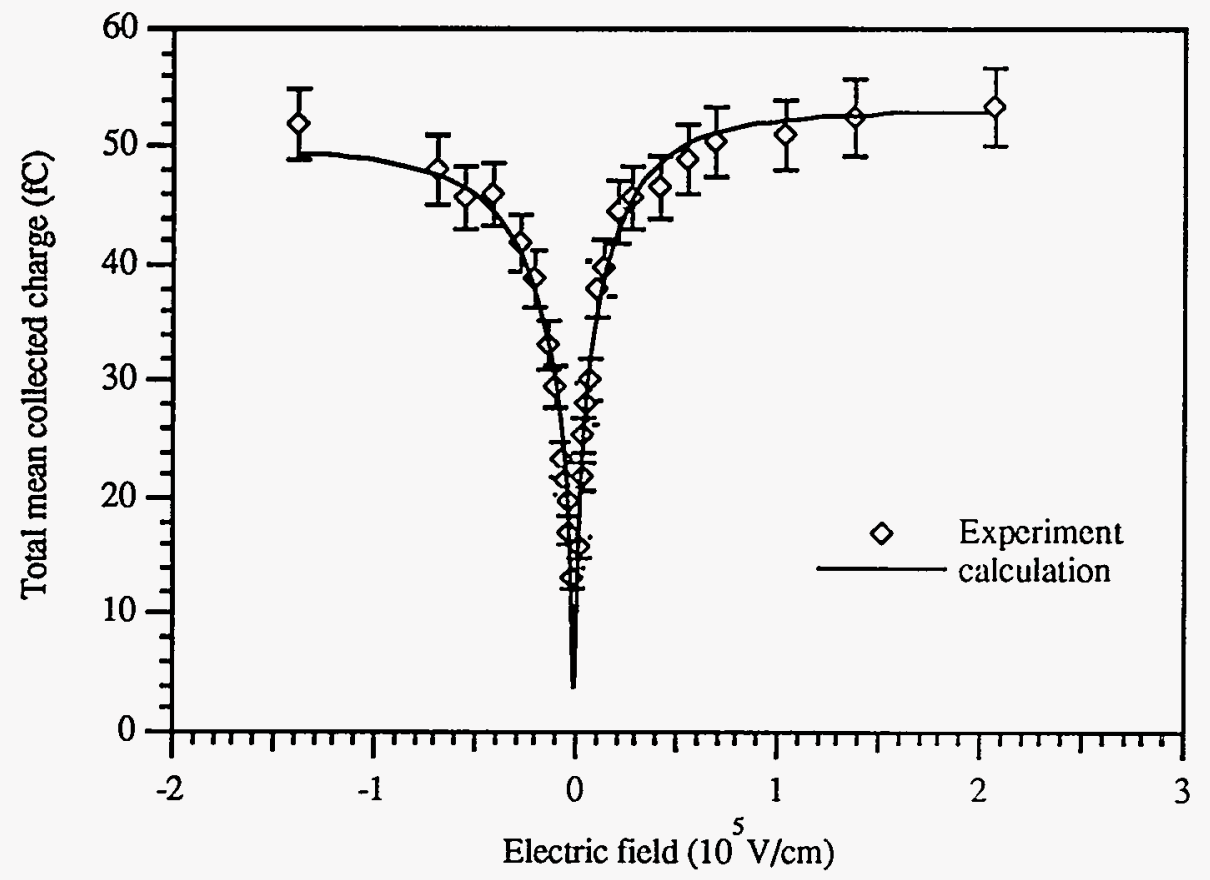

Figure 3.2.14: Comparison between the model and the experimental data for SYM14m using non-injecting contacts for both contacts. The mobility ratio was assumed to be 1.34 but $\varepsilon$ was free.

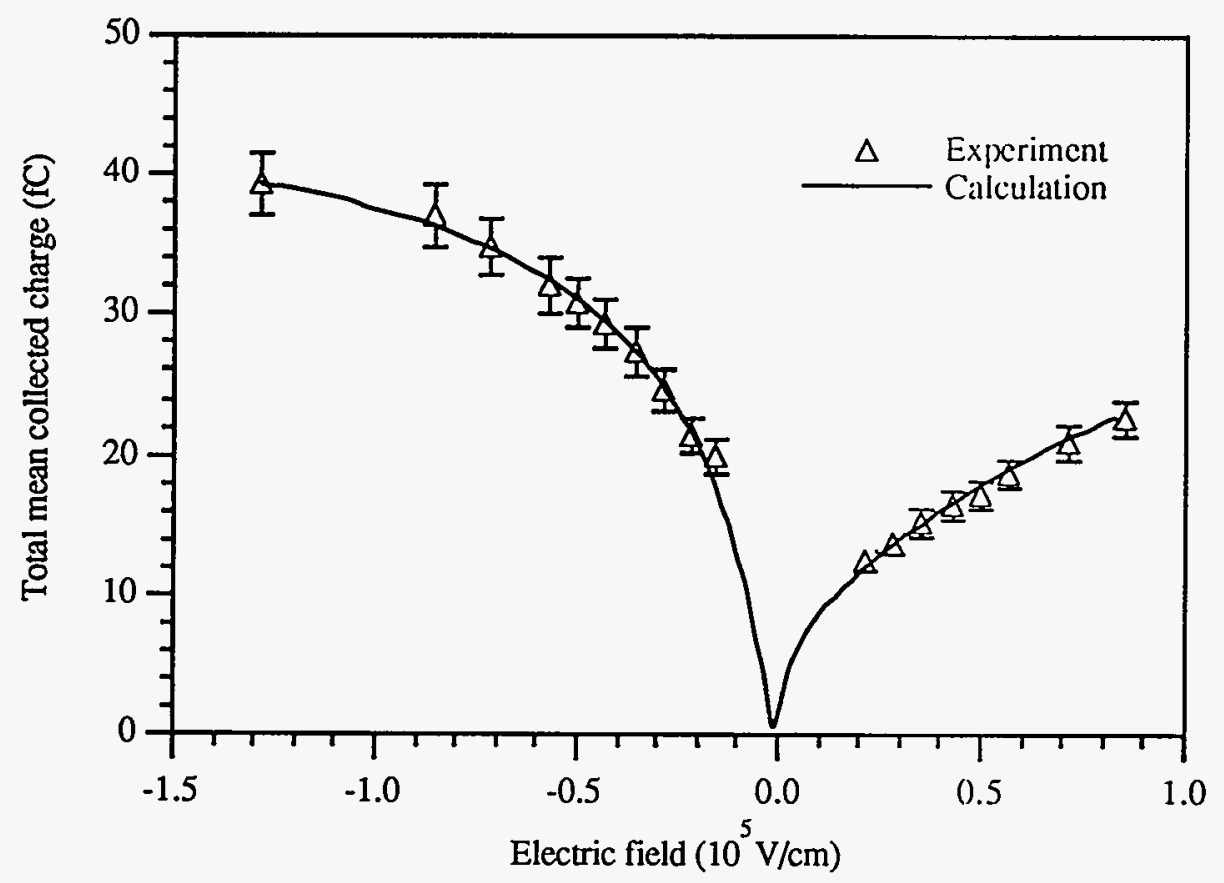

Figure 3.2.15: Comparison between the model and the experimental data for SYM70m using non-injecting contacts for both contacts. The mobility ratio was assumed to be 1.34 but $\varepsilon$ was free. 
Table 3.2.2: Estimated material parameters obtained from fitting the model to the experimental data for all detectors studied. The errors on the estimated parameters are just the estimated errors from non-linear least squares fitting. The contacts were assumed to be non-injecting. All material parameters were free to vary except for the electron to hole mobility ratio was fixed at 1.34 .

\begin{tabular}{|cccccc|}
\hline Detector & $\begin{array}{c}\mu_{\mathrm{e}} \\
\left(\mathrm{cm}^{2} / \mathrm{V}-\mathrm{sec}\right)\end{array}$ & $\begin{array}{c}\mu_{\mathrm{h}} \\
\left(\mathrm{cm}^{2} / \mathrm{V}-\mathrm{sec}\right)\end{array}$ & $\begin{array}{c}\tau_{\mathrm{e}} \\
(\mathrm{ps})\end{array}$ & $\begin{array}{c}\tau_{\mathrm{h}} \\
(\mathrm{ps})\end{array}$ & $\begin{array}{c}\varepsilon \\
(\mathrm{eV})\end{array}$ \\
\hline SYM14m & $825.0 \pm 230.0$ & $615.7 \pm 171.6$ & $38.1 \pm 16.8$ & $325.4 \pm 113.0$ & $13.1 \pm 1.0$ \\
SYM25m & $244.6 \pm 124.0$ & $182.5 \pm 92.5$ & $124.6 \pm 55.9$ & $433.0 \pm 203.0$ & $14.1 \pm 0.5$ \\
SYM70m & $226.3 \pm 152.0$ & $168.8 \pm 113.4$ & $247.7 \pm 144.0$ & $964.6 \pm 556.0$ & $16.7 \pm 1.0$ \\
\hline
\end{tabular}

Table 3.2.3: Estimated material parameters obtained from fitting the model to the experimental data for all detectors studied. The errors on the estimated parameters are just the estimated errors from non-linear least squares fitting. The contacts were assumed to be non-injecting. All five material parameters were free to vary in order to obtain these results

\begin{tabular}{|c|c|c|c|c|c|}
\hline Detector & $\begin{array}{c}\mu_{\mathrm{e}} \\
\left(\mathrm{cm}^{2} / \mathrm{N}-\mathrm{sec}\right)\end{array}$ & $\begin{array}{c}\mu_{\mathrm{h}} \\
\left(\mathrm{cm}^{2} / \mathrm{V}-\mathrm{sec}\right)\end{array}$ & $\begin{array}{c}\tau_{\mathrm{e}} \\
\text { (ps) }\end{array}$ & $\begin{array}{l}\tau_{\mathrm{h}} \\
(\mathrm{ps}) \\
\end{array}$ & $\begin{array}{c}\varepsilon \\
(\mathrm{eV})\end{array}$ \\
\hline SYM14m & $644.0 \pm 187.0$ & $1414 \pm 1550$ & $40.6 \pm 25.2$ & $156.0 \pm 178.0$ & $12.3 \pm 2.7$ \\
\hline SYM25m & $153.2 \pm 157.0$ & $320.1 \pm 209.0$ & $170.0 \pm 140.0$ & $270.0 \pm 160.0$ & $13.8 \pm 0.9$ \\
\hline SYM70m & $164.2 \pm 252.0$ & $186.9 \pm 132.0$ & $313.6 \pm 364.0$ & $882.6 \pm 544.0$ & $16.6 \pm 1.0$ \\
\hline
\end{tabular}


Table 3.2.6: Literature values for the average energy required to produce an electronhole pair in diamond.

Source

Dean, et al. [22]

Kennedy [23]

Canali, et al. [19]

Kozlov, et al. [24]

Konorova, et al. [25] $\varepsilon$ obtained with $\alpha$-particles, $\varepsilon$ obtained with $\beta$-particles,

$\mathrm{eV}$

$24.5 \pm 1.0$

$18.5 \pm 1.5$

$18.6 \pm 1.1$

13.25

13.56

$\mathrm{eV}$

consistent with the values associated with Canali, et. al. [21], Kozlov, et. al. [24] and Konorova, et. al. [25].

If we examine the $\mu-\tau$ product for holes, it is greater by a factor of about 2.6 as compared to the product for electrons and this is reflected in the total collected charge at a given electric field strength. With positive polarity, electrons must drift across the detector where they are trapped quickly and contribute relatively little to the collected charge. With negative polarity, the holes must drift over large distances with lower trapping in comparison to electrons. The dominance of the hole on the collected charge seems contradictory to the assumption that nitrogen is the main source of trapping in diamond. If this was true, we would expect the hole lifetime to be shorter than electron lifetime because nitrogen, with an energy level at $2 \mathrm{eV}$ below the conduction band edge [26], should be neutral donor initially and thereby capture holes [23]. This implies that these detectors may be trapping electrons preferentially at sites such as vacancies, in addition to the trapping of electrons by positively-charged nitrogen after the hole capture.

It has been speculated that vacancies may act as acceptors [27] in diamond.

Urlau, et. al., [28] have suggested the existence of an electron trap at $3.3 \mathrm{eV}$ above the 
valence band for type IIa diamond. In the limit of thermal equilibrium, the electron lifetimes listed in Table 3.2.1 can result if the acceptor concentration is on the order of $7 \times 10^{16} \mathrm{~cm}^{-3}$ and if the electron capture cross section is about $10^{-14} \mathrm{~cm}^{2}$, the latter being a conservative estimate for a positively-charged center [29]. Clearly, even small concentrations of acceptors may cause the electron lifetime to be short.

In Fig. 3.2.16, comparisons between experimental and calculated ratios of the total mean collected charge with positive polarity to that with negative polarity are shown.. For all three detectors the agreement between calculation and experiment is reasonable at absolute values of electric fields of $>0.2 \times 10^{5} \mathrm{~V} / \mathrm{cm}$. There is a greater difference in the absolute magnitude of the ratio at fields below $0.2 \times 10^{5} \mathrm{~V} / \mathrm{cm}$. This may be due to the non-constant carrier lifetimes as a function of applied electric field. The field-dependence of the carrier lifetimes has been seen by Pan [30] for the field in the range $0-5 \times 10^{3} \mathrm{~V} / \mathrm{cm}$ where larger lifetimes exist at lower fields in comparison to the higher fields.

The experimental distributions for both SYM14m and SYM25m show significant changes in slopes for fields less than about $0.2 \times 10^{5} \mathrm{~V} / \mathrm{cm}$. According to the model, a change in slope is expected under some cases. It is due primarily to the interplay between variation in detector thickness that increases the drift dimensions for charge collection of both electrons and holes, and the difference in the transport properties of the two carriers. For SYM14m, the calculated ratio shows this effect but it is damped relative to the experimental data. For SYM70m, the trend in the data is not reflected in the model calculation at all.

The model indicates that when a minimum in the collected charge ratio is predicted, its magnitude is primarily dependent upon the carrier lifetimes. On the other hand, when a maximum is predicted, its location is determined primarily by the electric field. 
Finally, in Fig. 3.2.17, ratios of $\varepsilon$ to the bandgap for different detector materials are shown along with least-squares-fit to an equation of the form $y=a+b / x$. The figure clearly shows a general tendency for decreasing $\varepsilon$ with increasing band gap. The ratio for diamond was calculated using $5.5 \mathrm{eV}$ for the band gap and $\varepsilon$ from the model fit to SYM25m. The figure also includes Klein's model [31] where an incident particle energy is lost by producing plasmons and each plasmon decays with formation of a single electron-hole pair. One of the caveats in Klein's model is that the phonons are assumed to play a negligible role in the ionization. The coefficients from the fit to the available data appear to be in a reasonable agreement with Klein's model. 


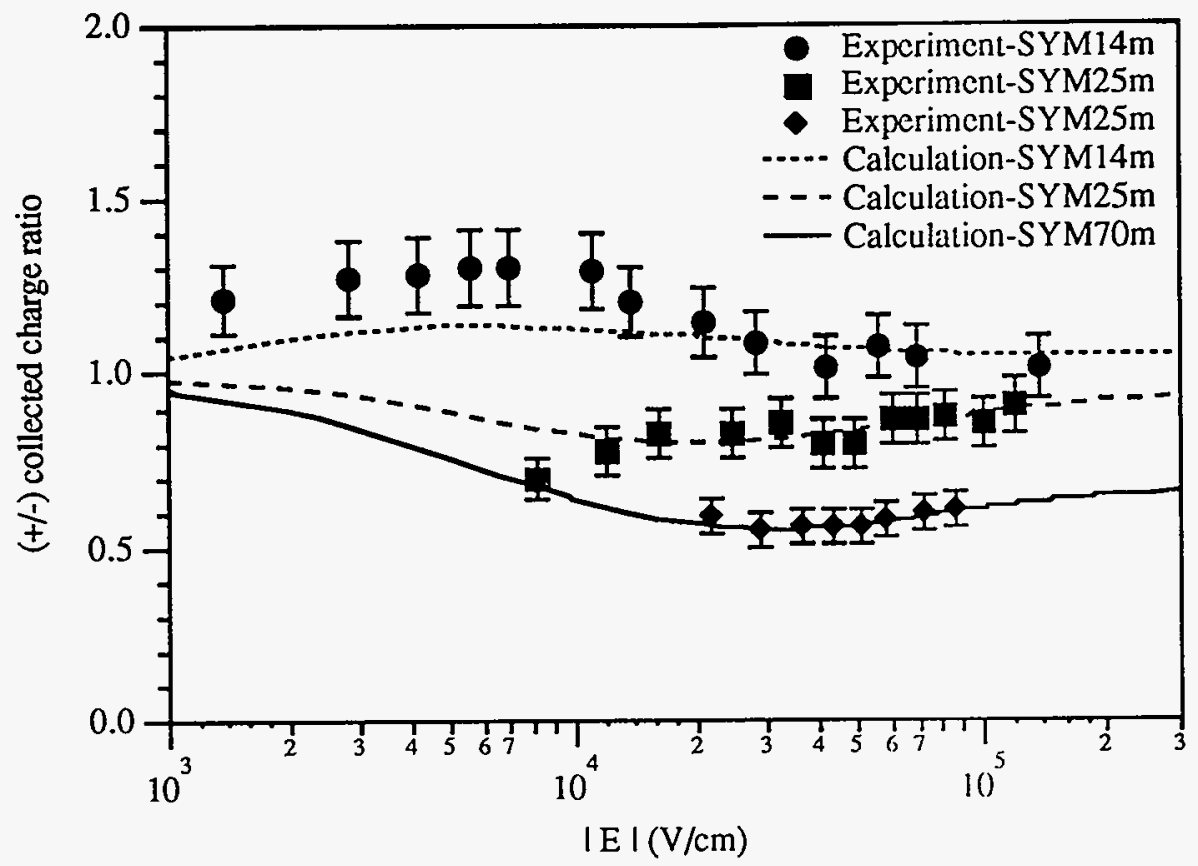

Figure 3.2.15: Comparison between experimental and calculated ratios of the mean collected charge for the positive polarity to that for negative polarity as a function of the absolute value of the applied electric field, IEI. 


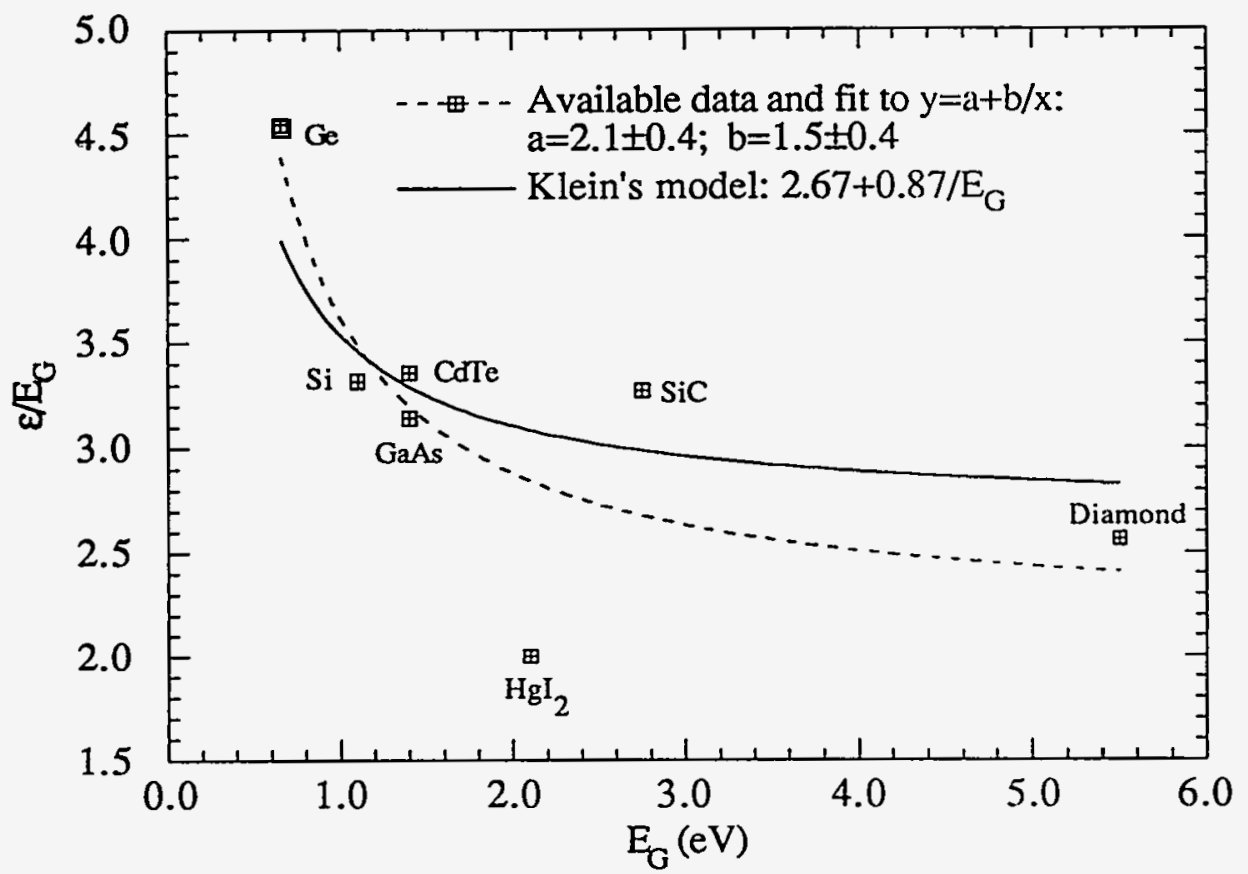

Figure 3.2.16: Ratio of mean energy required to produce an electron-hole pair, $\varepsilon$, to band gap, $\mathrm{E}_{\mathrm{G}}$, as a function of $\mathrm{E}_{\mathrm{G}}$. As the band gap increases, the ratio correspondingly decreases. The data were taken from Ref. 32. Klein's model [ 36] is also plotted. 


\subsection{Detector resolution}

As indicated previously, the fractional resolution of the three diamond detectors studied is much greater than expected if the statistics of charge production was the main source of variation in the collected charge. In the latter case, the fractional resolution, expressed as the ratio of the of FWHM of a Gaussian to the mean charge in the limit of a Poisson distribution and full charge collection, is [33]

$$
D R=2.355 \sqrt{\frac{1}{N_{I}}}
$$

where $N_{I}=$ the mean initial number of carriers produced, $\frac{E_{o}}{\varepsilon}$

$$
\begin{aligned}
& E_{o}=\text { energy deposited in the detector } \\
& \varepsilon=\text { mean energy needed to create an electron-hole pair. }
\end{aligned}
$$

This assumes that the production of individual ion pairs is independent of the production of all others. In reality, the inherent detector fractional resolution is better than that calculated with Equ. 3.3.1 for truly monoenergetic events because of the correlation in production of charge carriers. Such correlations are usually described by the Fano factor, F [34], and the expected fractional resolution is given by

$$
D R=2.355 \sqrt{\frac{F}{N_{I}}}
$$

The Fano factor varies from 0 to 1 where 1 represents the Poisson limit. For Si, Fano factors reported in the literature lie in the range 0.08 to $0.5[40,35]$. Fano factors have been calculated by Alkhazov, et al. [36], for various semiconductor and gas radiation detectors, and all are significantly less than unity. As a result, the simple Poisson limit $(\mathrm{F}=1)$ can be considered to be a very conservative estimate of the detector resolution if it is solely due to the statistics of carrier production. 
No measurement or estimate of the Fano factor for diamond has been made. The fractional resolution in the Poisson limit with $\varepsilon=14.1 \mathrm{eV}$ is calculated to be about 0.004 . Experimentally, the best fractional resolution found with any of the three detectors studied at the highest applied electric field is about 0.17 .

Obviously the statistics of charge production can be but a small contributor to the overall detector resolution if all charges are collected. In an attempt to understand the enormous difference between expected and measured resolution, the contribution of incomplete charge collection has been considered.

\subsubsection{Resolution effects of incomplete charge collection}

The effect of incomplete charge collection on fractional resolution has been considered by Dearnaley and Northrop [37] and Day, et. al [381. In the analysis by Dearnaley and Northrop, the case of injecting contacts has been treated and Day, et. al have considered the case of non-injecting contacts. For the case of injecting contacts, the fractional resolution, DR, is given by

$$
D R=2.355 \sqrt{\frac{1}{N_{I}}}
$$

This is exactly the fractional resolution obtained from considering charge production alone with $\mathrm{F}=1$. Therefore, the total fractional resolution (charge production and collection) for the case of injecting contacts can be expressed as

$$
D R=2.355 \sqrt{\frac{(F+1)}{N_{I}}}
$$

If the diamond detectors studied here had injecting contacts, the total fractional resolution can be estimated to be about 0.006 in the Poisson limit. Again, this value is much less than the resolution of 0.17 and thus can be considered additional evidence supporting the 
conclusion in Sec. 3.3.2 that the contacts used with the present detectors are of the noninjecting type.

In the case of non-injecting contacts, Day, et. al. have treated the case of incomplete charge collection when a detector is uniformly irradiated. In their derivation the total mean collected charge, $\bar{q}$, was derived and then the variance of the mean was found by use of the definition

$$
\sigma^{2}=\left(\overline{q^{2}}\right)-(\bar{q})^{2}
$$

where the mean charge $\bar{q}$ is given by Equ. 3.2.23 and is derived by considering the spatial dependence of the collected charge (see Sec. 3.2). Thus

$$
\begin{aligned}
& \qquad \overline{q^{2}}=\frac{1}{L} \int_{0}^{L}\left(q_{e}(x)+q_{h}(x)\right)^{2} d x \\
& \text { where } q_{e}(x)=\frac{e N_{l} \lambda_{e}}{L}\left[1-e^{-x / \lambda_{e}}\right] \\
& \text { and } q_{h}(x)=\frac{e N_{l} \lambda_{h}}{L}\left[1-e^{-x / \lambda_{h}}\right]
\end{aligned}
$$

The variance resulting with the uniform illumination of a detector case is then found as

$$
\sigma^{2}=\left(\frac{e^{2} N_{I}^{2}}{L^{3}}\right)\left[\begin{array}{l}
\frac{\left(\lambda_{e}^{3}+\lambda_{h}^{3}\right)}{2}-2\left(\lambda_{e}+\lambda_{h}\right)\left(\lambda_{e}^{2}+\lambda_{h}^{2}\right)-\frac{\lambda_{e}^{3}}{2} e^{-2 L / \lambda_{e}} \\
+\frac{2 \lambda_{e}^{2} \lambda_{h}^{2}}{\left(\lambda_{e}-\lambda_{h}\right)} e^{-L / \lambda_{e}}+2 \lambda_{e}^{2}\left(\lambda_{e}+\lambda_{h}\right) e^{-2 L / \lambda_{e}} \\
-\frac{\lambda_{h}^{3}}{2} e^{-2 L / \lambda_{h}}+L\left(\lambda_{e}+\lambda_{h}\right)^{2}-\frac{2 \lambda_{e}^{2} \lambda_{h}^{2}}{\left(\lambda_{e}-\lambda_{h}\right)} e^{-L / \lambda_{e}} \\
+2 \lambda_{h}^{2}\left(\lambda_{h}+\lambda_{e}\right) e^{-L / \lambda_{h}}
\end{array}\right]-(\bar{q})^{2}
$$

In the present case, where $\alpha$-particles enter the detector through one of the electrical contacts, and when the average trajectory is parallel to the applied electric field, 
significant spatial non-uniformities in chärge production are present. As a result, the expressions for $q_{e}(x)$ and $q_{h}(x)$ become

$$
\begin{aligned}
& q_{e}(x)=\frac{e}{\varepsilon} \int_{0}^{x}\left(\frac{d E}{d x^{\prime}}\right) e^{-x^{\prime} / \lambda_{e}} \frac{d x^{\prime}}{L} \\
& q_{h}(x)=\frac{e}{\varepsilon} \int_{0}^{x}\left(\frac{d E}{d x^{\prime}}\right) e^{-x^{\prime} / \lambda_{k}} \frac{d x^{\prime}}{L}
\end{aligned}
$$

where $\left(\frac{d E}{d \dot{x}}\right)$ is the stopping power for the $\alpha$-particles. Similar to the approximation used in Sec. 3.2.1, we assume first a constant stopping power, i.e., $\int \frac{d E}{d x} d x=R\left\langle\frac{d E}{d x}\right\rangle$, to arrive at a closed form solution analogous to Equ. 3.3.9. With this assumption, equations 3.3.10 and 3.3.11 reduce to equations 3.3.7 and 3.3.8. The mean squared collected charge given in Eq. 3.3.6 must now be written as

$$
\overline{q^{2}}=\frac{1}{R} \int_{0}^{R}\left(q_{e}(x)+q_{h}(x)\right)^{2} d x
$$

With use of the value of $\bar{q}$ from the summation of the terms given in equations 3.3 .21 and 3.2.22, integration of Eq. 3.3.12 leads to the following variance from charge collection;

$$
\sigma^{2}=\left(\frac{e^{2} N_{l}^{2}}{R L^{2}}\right)\left[\begin{array}{l}
\frac{\lambda_{h}^{3}}{2}-2 \lambda_{h}^{2}\left(\lambda_{e}+\lambda_{h}\right)-\frac{\lambda_{e}^{3}}{2} e^{-2 L / \lambda_{c}} \\
+\frac{2 \lambda_{e}^{2} \lambda_{h}^{2}}{\left(\lambda_{e}-\lambda_{h}\right)} e^{-L / \lambda_{e}}+2 \lambda_{e}^{2}\left(\lambda_{e}+\lambda_{h}\right) e^{-L / \lambda_{c}} \\
-\frac{\lambda_{h}^{3}}{2} e^{-2 R / \lambda_{h}}-2 \lambda_{e}^{2}\left(\lambda_{e}+\lambda_{h}\right) e^{-(L-R) / \lambda_{e}} \\
+\frac{\lambda_{e}^{3}}{2} e^{-2(L-R) / \lambda_{e}}+2 \lambda_{h}^{2}\left(\lambda_{e}+\lambda_{h}\right) e^{-R / \lambda_{h}} \\
-\frac{2 \lambda_{e}^{2} \lambda_{h}^{2} e^{-(L-R) / \lambda_{e}} e^{-R / \lambda_{h}}}{\left(\lambda_{e}-\lambda_{h}\right)}+R\left(\lambda_{e}+\lambda_{h}\right)^{2}
\end{array}\right]-(\bar{q})^{2}
$$


We can now express the expected fractional resolution as

$$
D R=\frac{2.355 \sqrt{\sigma^{2}+\sigma_{p r o d}^{2}}}{\bar{q}}
$$

where $\sigma_{\text {prod }}^{2}=$ variance due to carrier production, $e F \frac{E_{0}}{\varepsilon}$

In the limit that $\frac{\lambda_{e}}{L} \rightarrow 0$ and $\frac{\lambda_{h}}{L} \rightarrow 0, \sigma^{2}$ and $\bar{q}$ approach zero, as expected, and DR approaches infinity because $\sigma_{\text {prod }}^{2}$ is constant as long as $\mathrm{F}$ is finite. In the limit that $\frac{\lambda_{e}}{L} \rightarrow \infty$ and $\frac{\lambda_{h}}{L} \rightarrow \infty, \sigma^{2}$ again approaches zero and DR approaches the value given by

Equ. 3.3.2. In this case all charges are collected, and it is obvious that large mean free paths are required to minimize resolution effects of incomplete charge collection if the detector contacts are non-injecting.

We have used the result of Eq. 3.3.13 to calculate the expected fractional resolution (Equ. 3.3.14) as a function of bias polarity and electric field. For brevity, we will refer to the low-field $\mu_{h} \tau_{h}$ and the low-field $\mu_{e} \tau_{e}$ as just $\mu_{h} \tau_{h}$ or $\mu_{e} \tau_{e}$, respectively'. In Fig. 3.3.1 we show the fractional resolution assuming for the cases $\frac{\mu_{e} \tau_{e}}{\mu_{h} \tau_{h}}=0.1,1$, assuming $\mu_{h} \tau_{h}=3 \times 10^{-8} \mathrm{~cm}^{2} / \mathrm{V}, \mathrm{F}=0.5,1$, and a detector thickness $\mathrm{L}=25 \mu \mathrm{m}$. When $\mu_{k} \tau_{k}=\mu_{e} \tau_{e}$ (Fig. 3.3.1), an asymmetry in the fractional resolution is seen at high fields. This is due to the non-uniform excitation caused by the $\alpha$-particles $(R \approx 0.5 \mathrm{~L})$ and the differences in the effective masses of electrons and holes. For both Fano factors, the shapes of DR profiles do not change but the absolute magnitudes because $\sigma_{\text {prod }}^{2}$ is independent of the applied field. In Fig. 3.3.2, the ratio of $\sigma^{2}$ to $\sigma_{\text {prod }}^{2}$ (VR) as a function of bias polarity and applied field is shown for both Fano factors. Under the given material and operational parameters, $\sigma_{\text {prod }}^{2}$ is greater than $\sigma^{2}$ over the entire range of fields considered. With an order of magnitude difference in the low-field $\mu \tau$-products (Fig. 3.3.3), the asymmetry is greatly magnified. With positive polarity the resolution is worsened by a factor of about 2.5 compared to negative polarity. As will be shown, this 


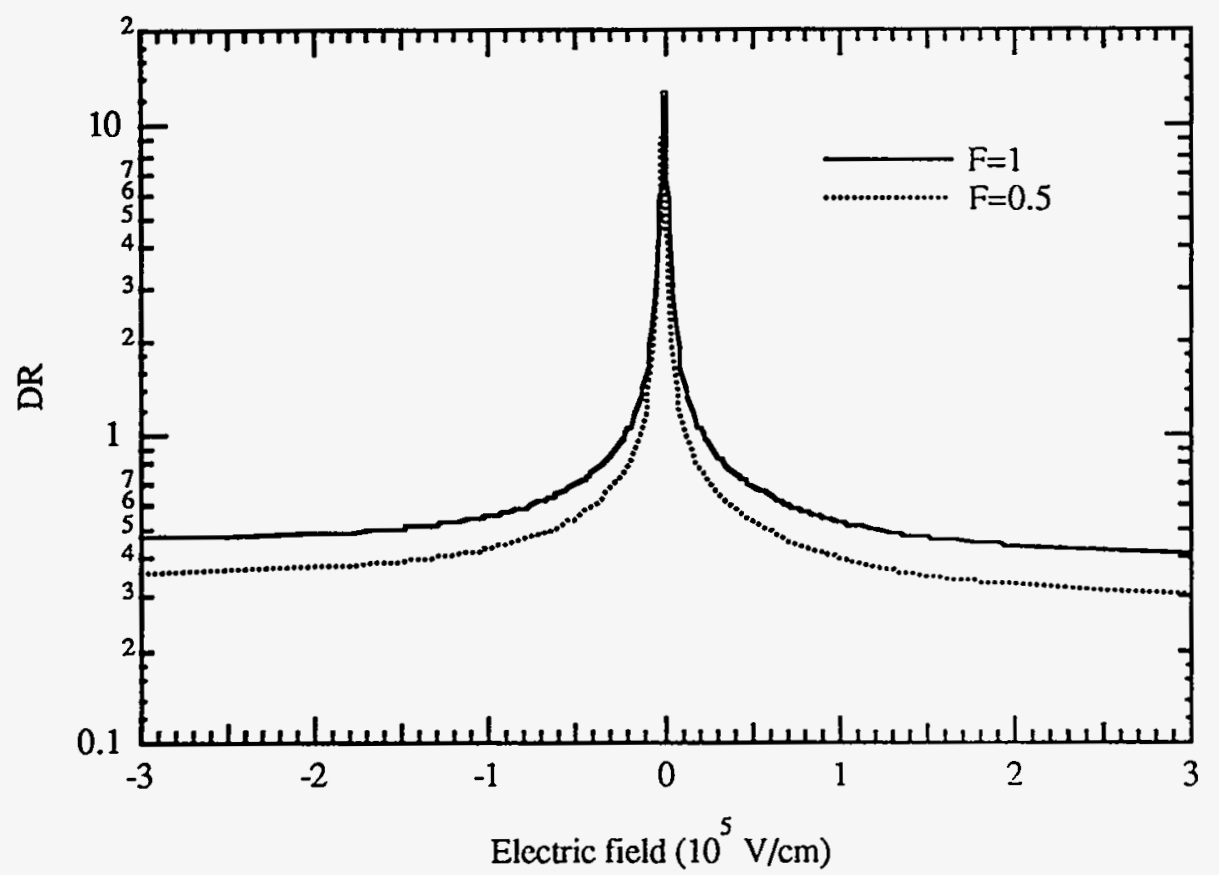

Figure 3.3.1: Calculated fractional resolution (DR) as a function of bias polarity, applied electric field and the Fano factor $(F)$. The detector width is $\mathrm{L}=25 \mu \mathrm{m}$ and $\mu_{h} \tau_{h}=\mu_{e} \tau_{e}=3 \times 10^{-8} \mathrm{~cm}^{2} / \mathrm{V}$. 


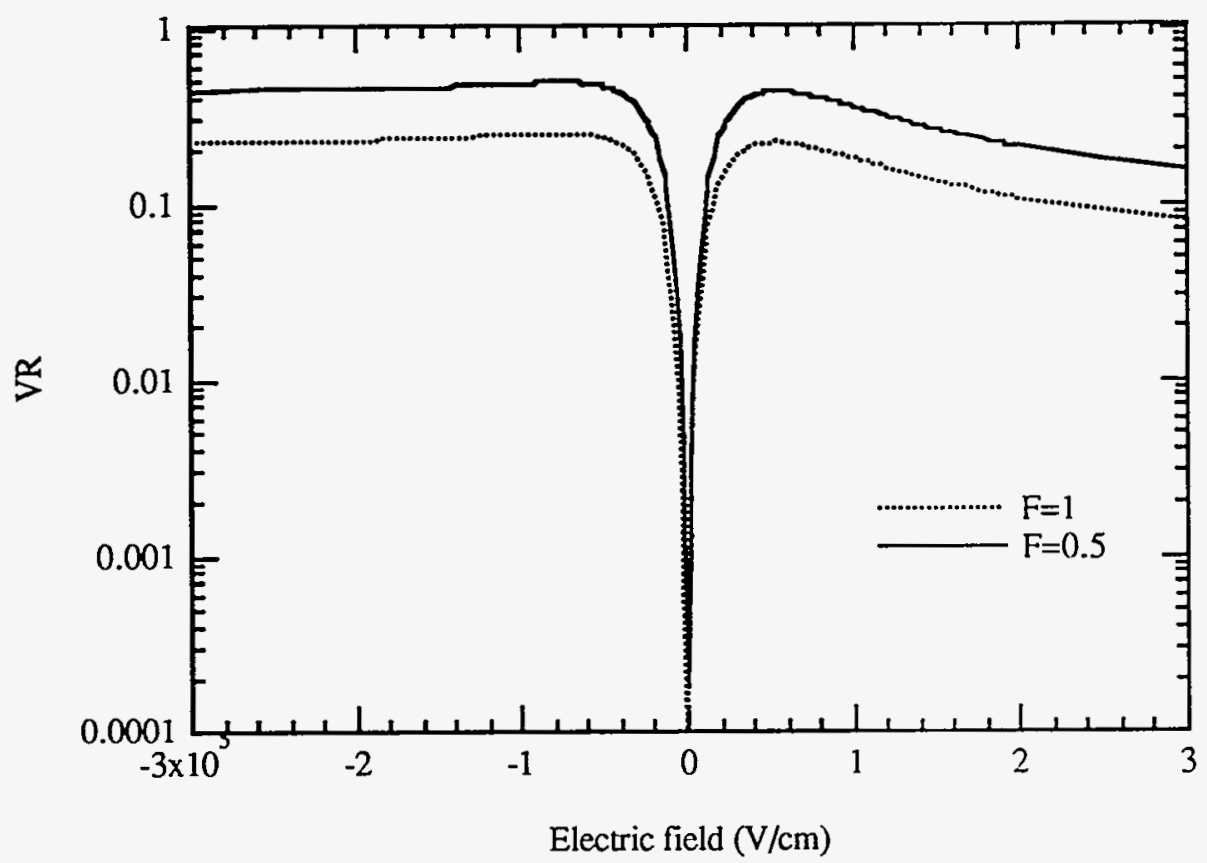

Figure 3.3.2: Ratio of variance due to charge collection to variance due to charge production, VR, as a function of bias polarity, applied electric field and the Fano factor $(F)$. The detector width is $\mathrm{L}=25 \mu \mathrm{m}$ and $\mu_{h} \tau_{h}=\mu_{e} \tau_{e}=3 \times 10^{-8} \mathrm{~cm}^{2} / \mathrm{V}$. 


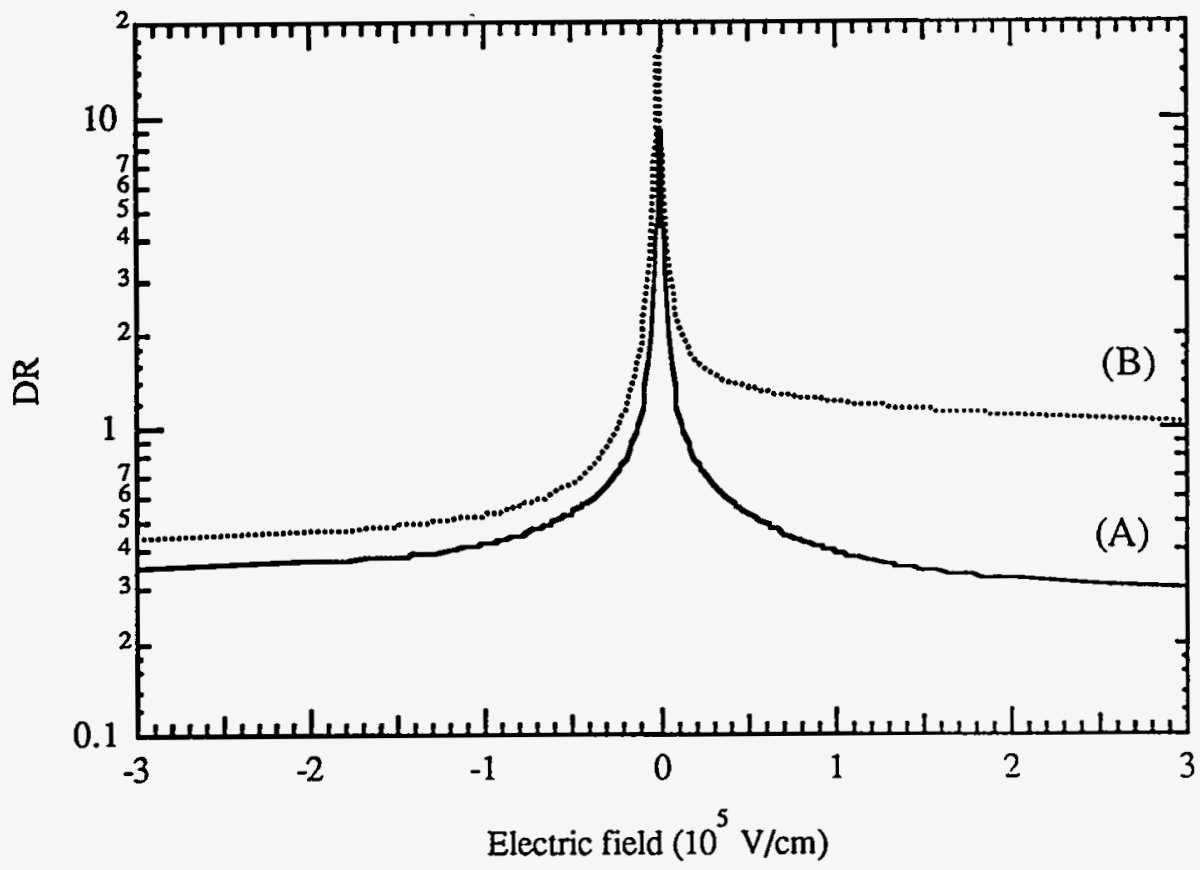

Figure 3.3.3: Calculated fractional resolution (DR) as a function of bias polarity, applied electric field; (A) calculated DR with $\mu_{h} \tau_{h}=\mu_{e} \tau_{e}$; (B) calculated DR with $\mu_{e} \tau_{e}=0.1 \mu_{h} \tau_{h}$. The detector width, $\mu_{h} \tau_{h}$ and the Fano factor are $\mathrm{L}=25 \mu \mathrm{m}, 3 \times 10^{-8} \mathrm{~cm}^{2} / \mathrm{V}$ and $\mathrm{F}=0.5$, respectively.

type of asymmetry is clearly evident in the measured resolutions of all three detectors. Nevertheless, it is clear from Fig. 3.3.3 that if incomplete charge collection is serious, an appropriate choice of operating polarity will allow at least one carrier to be collected to a reasonable degree and therefore lead to improved resolution.

The effect of low-field carrier mobility and lifetime on the detector resolution has also been examined. For these calculations, we assumed $\mu_{h} \tau_{h}=\mu_{e} \tau_{e}=\mu \tau$ and $\mathrm{F}=0.5$. As stated in Sec. 3.2, the carrier lifetime is assumed to be independent of electric field whereas the carrier mobility was taken to be field-dependent. The effect of variation in carrier lifetime is shown in Fig. 3.3.4 where the low-field $\mu \tau$-products were varied by changing the magnitude of the carrier lifetime with the low-field mobility held constant. 
The effect of variation in the carrier mobility is shown in Fig. 3.3.5 where the low-field $\mu \tau$-products were varied by changing the value of carrier mobility with the carrier lifetime held constant. We can clearly see that the detector resolution over the range of electric fields considered is better in the case of increasing lifetime as compared to increasing carrier mobility. This is due to the fact that the carrier drift velocity begins to saturate beyond about $1 \times 10^{4} \mathrm{~V} / \mathrm{cm}$, whereas the carrier lifetime is assumed to be fieldindependent. Also, asymmetry in the fractional resolution is much greater when the mobility is varied, again, due to saturation in the carrier drift velocity.

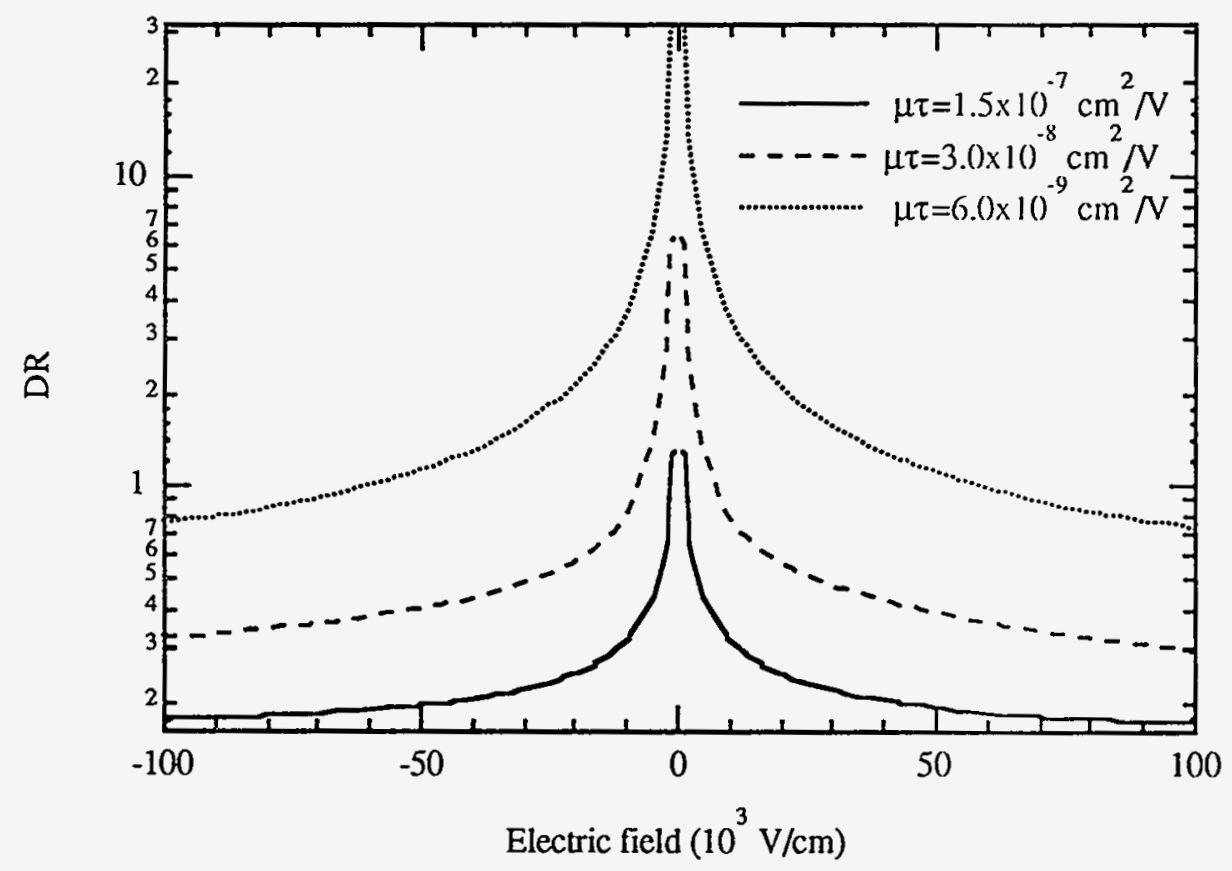

Figure 3.3.4: Calculated fractional resolution (DR) as a function of applied electric field and bias polarity with $\mu_{h} \tau_{h}=\mu_{e} \tau_{e}=\mu \tau . \mu \tau$ was varied by changing the carrier lifetime. The detector width and the Fano factor are $L=25 \mu \mathrm{m}$ and $F=0.5$, respectively. 


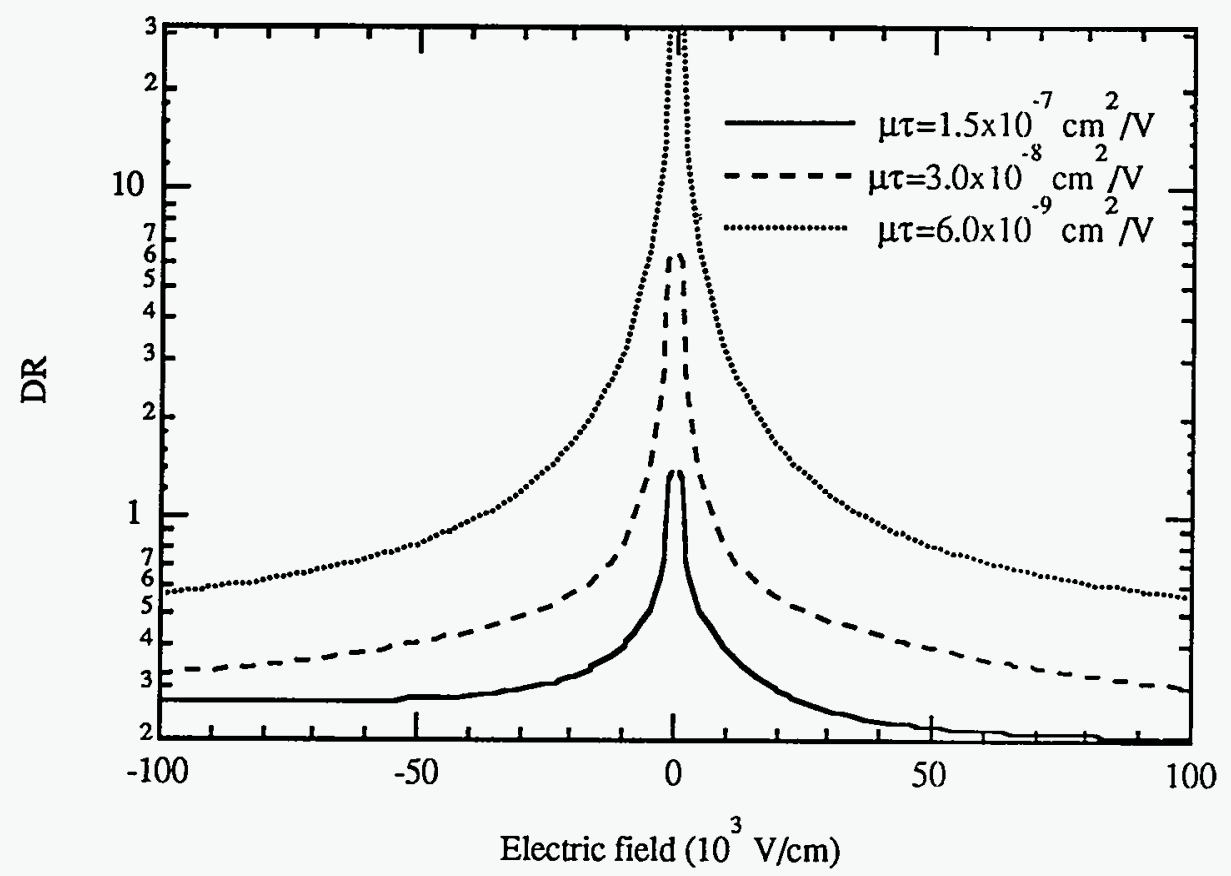

Figure 3.3.5: Calculated fractional resolution (DR) as a function of applied electric field and bias polarity with $\mu_{h} \tau_{h}=\mu_{e} \tau_{e}=\mu \tau . \mu \tau$ was varied by changing the low-field carrier mobility. The detector width and the Fano factor are $\mathrm{L}=25 \mu \mathrm{m}$ and $\mathrm{F}=0.5$, respectively.

In Fig. 3.3.6, the calculated fractional resolution is shown as a function of detector thickness, $\mathrm{L}$, when $\mu_{e} \tau_{e}=\mu_{h} \tau_{h}=3 \times 10^{-8} \mathrm{~cm}^{2} / \mathrm{V}$. As expected, the fractional resolution is significantly better when the detector thickness is the smallest because the fraction of the initial charge that is collected is the largest. As the detector thickness increases, the resolution first degrades rapidly because of reduced total charge collection. In the limit that $\mathrm{L} \rightarrow \infty, \bar{q}$ approaches zero because the mean free paths of both electrons and holes become small compared to L. At this limit, DR approaches infinity, as expected, because $\sigma_{\text {prod }}^{2}$ is constant. As expected, when $\mathrm{R}$ is identical to $\mathrm{L}$ (here taken as $14.2 \mu \mathrm{m}$ ), the fractional resolution profile is symmetric with respect to bias polarity.

While the discussion above provides a guide to interpreting the experimental data, detailed comparisons require use of a realistic representation of the stopping power of the 


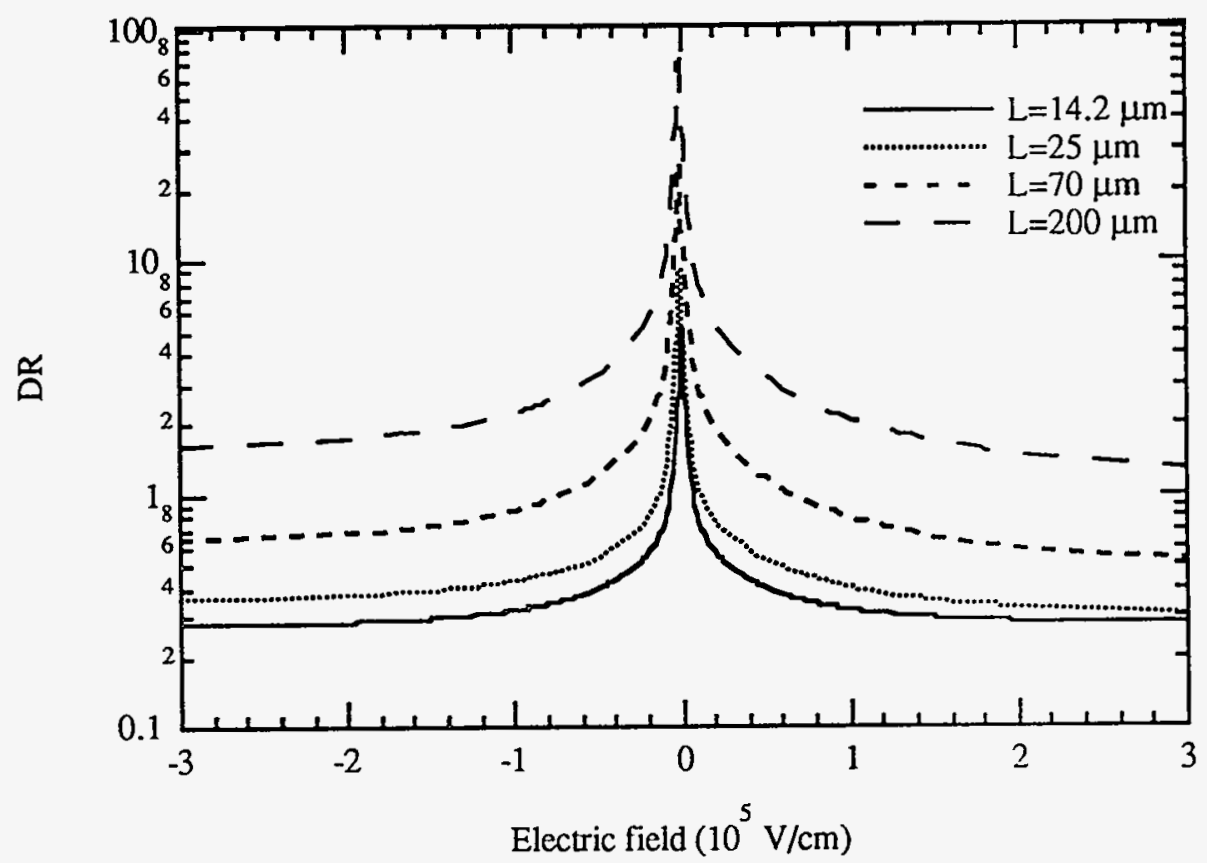

Figure 3.3.6: Calculated fractional resolution (DR) as a function of applied electric field, bias polarity and the detector width, L. The Fano factor is taken to be 0.5 .

$\alpha$-particles. For this purpose, the stopping powers shown in Fig. 3.2.2 were used in Eq. 3.3.10 - 3.3.11. Numerical integration of Eq. 3.3.12 then yielded the expected fractional resolutions. In Fig. 3.3.7, the resolution profiles calculated with constint and nonconstant stopping powers are shown using $\mu_{h} \tau_{h}=\mu_{e} \tau_{e}=3 \times 10^{-8} \mathrm{~cm}^{2} / \mathrm{V}$. We can observe a significant difference in the absolute value of the fractional resolutions but the shapes of the profiles are approximately the same.

To quantify the difference between the two calculations, the relative difference, FD, defined as

$$
F D=\frac{|D R|_{\text {TRIM }}-\left.D R\right|_{\text {const }} \mid}{\left.D R\right|_{\text {TRMM }}}
$$


was calculated and is shown in Fig. 3.3.8. For electric fields in the range $\pm\left(0.07 \times 10^{5}\right.$ $\left.3 \times 10^{5}\right) \mathrm{V} / \mathrm{cm}$, the difference between the two calculation is between $0.32-0.45$ with the largest differences appearing at the lower fields. The largest difference is about 0.45 . These errors between the two approaches appear to be large. Therefore, in comparing measured and calculated fractional resolution, non-constant stopping power will be used. 


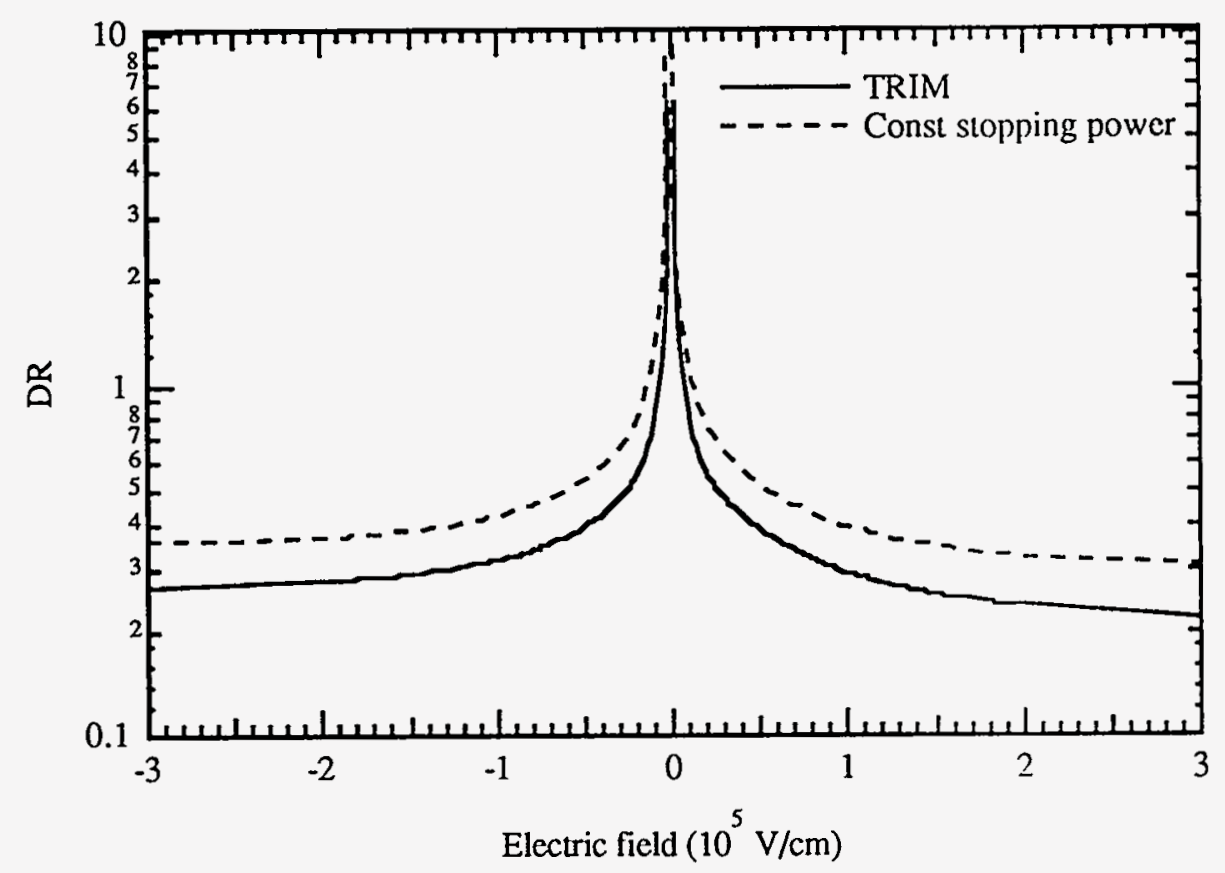

Figure 3.3.7: Comparison between fractional resolution calculated with constant and non-constant stopping powers for $\alpha$-particles. The parameters assumed in the calculations are $\mathrm{L}=25 \mu \mathrm{m}, \mathrm{R}=14.2 \mu \mathrm{m}, \mathrm{F}=0.5$ and $\mu_{e} \tau_{e}=\mu_{h} \tau_{h}=3 \times 10^{-8} \mathrm{~cm}^{2} / \mathrm{V}$. 


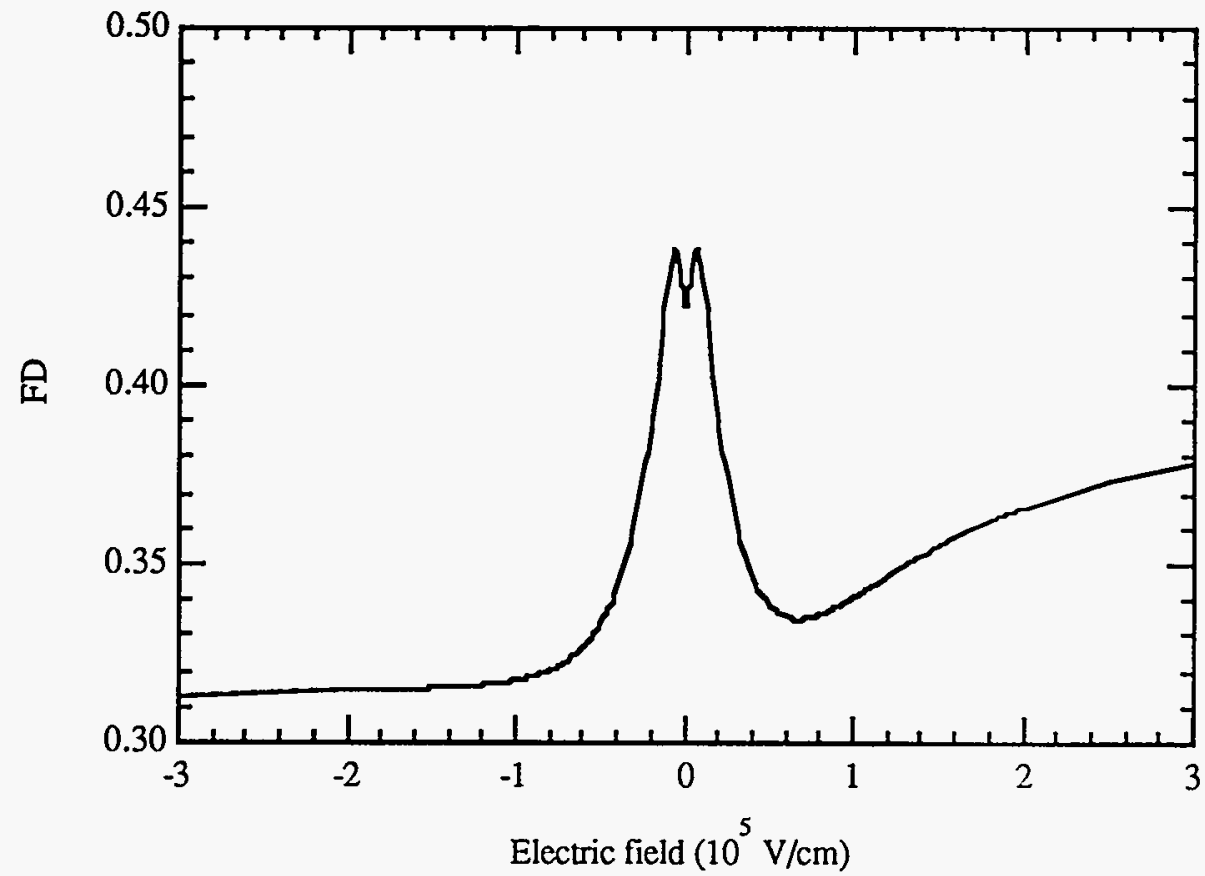

Figure 3.3.8: Relative difference between non-constant and constant stopping powers as a function of electric field. The parameters assumed in the calculations are $\mathrm{L}=25 \mu \mathrm{m}, \mathrm{R}=14.2 \mu \mathrm{m}, \mathrm{F}=0.5$ and $\mu_{e} \tau_{e}=\mu_{h} \tau_{h}$ $=3 \times 10^{-8} \mathrm{~cm}^{2} / \mathrm{V}$.

3.3.2 Comparison between calculated and experimental detector resolutions

In this section, the comparisons between calculated and experimental detector fractional resolutions are presented. The comparisons were performed with two purposes in mind. The first was to understand the large fractional resolutions that were found experimentally and the second was to examine the possibility of using the fractional resolution to estimate mean carrier transport properties. No model fitting was performed but the transport properties obtained in Sec. 3.2.2 by fitting were used to calculate the fractional resolution profiles. In these calculations, only four parameters, $\mu_{\mathrm{e}}, \tau_{\mathrm{e}}, \mu_{\mathrm{h}}$, and $\tau_{\mathrm{h}}$ are required. 
In an attempt to minimize differences between the calculated and experimental resolutions, a series of profiles was calculated by varying transport properties independently by a fraction $f$ of their standard deviations shown in Table 3.2.1. Values of $\mathrm{f}$ were chosen randomly in the range -1 to +1 , a profile was calculated and the sum of the squares of deviation between the experimental and calculated resolutions was determined. The process was repeated to find the values of $f$ that minimized the sum of the deviation. The values of $f$ that resulted in the best fits to experiment are given in Table 3.3.1 and the resolution profiles obtained from the best fit are included in the figures discussed below.

Fig. 3.3.9 shows the comparison between measured and calculated for SYM25m using a Fano factor of 0.3 . For this detector, the calculated fractional resolution with the estimated mean transport properties from Table 3.2.2 agrees well over the range of applied fields with positive. The calculated fractional resolution for the negative polarity is greater by factors $<1.4$. The quantitative and qualitative agreements appear to be reasonable over the entire range of the applied fields. As mentioned previously, asymmetry in the fractional resolution profile with respect to the bias polarity is clearly seen in both the measured and the calculated fractional resolution profiles. By modifying the estimated mean by a fraction, $f$, between $\pm(0.63-0.98)$ of the standard deviation, the agreement between model and experiment appears to be excellent.

In figures 3.3.10 and 3.3.11, the comparisons between calculated and experimental fractional resolutions for detector SYM14m are shown with Fano factors of 0 and 0.3 . By using the mean values, the expected fractional resolution is calculated to be larger than experiment by a factor of about 2 over the entire range of applied electric field used in the experiment with the Fano factor of 0.3. Even with the Fano Factor of 0 , which implies that no fluctuation in the charge production exists, the calculated resolution is greater by a factor of about 2 . 


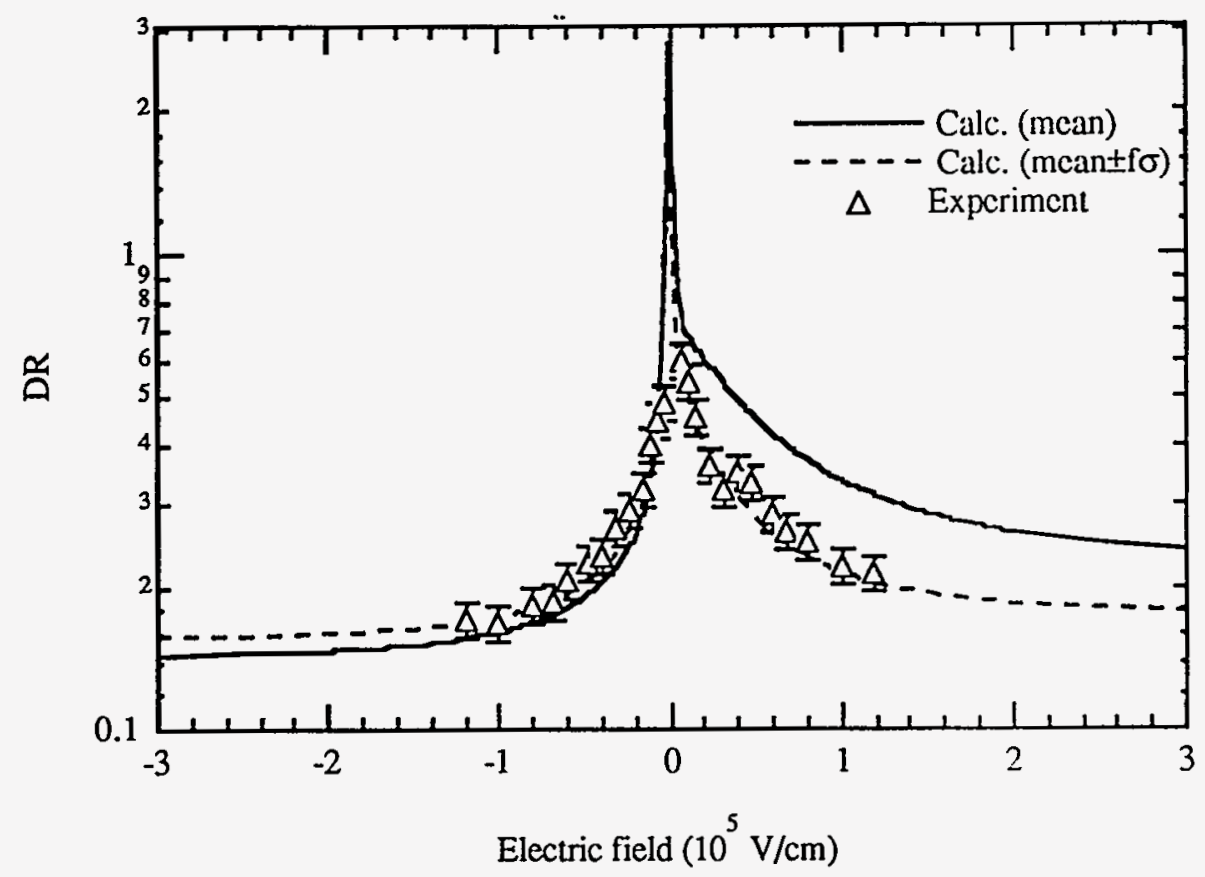

Figure 3.3.9: Comparison between calculated and experimental fractional resolution for detector SYM25m. ( ------- ) calculated width mean values of carrier mobilities and lifetimes from Table 3.2.1. $\left(\_\right.$) calculated by modifying the mean by the quantity fo where the $\mathrm{f}$ are given in Table 3.3.1. The Fano factor was taken to be 0.3 .

By modifying the transport properties within one standard deviation from the mean, better quantitative agreement between measured and calculated fractional resolutions is seen but the discrepancy is still very large at fields $<0.5 \times 10^{5} \mathrm{~V} / \mathrm{cm}$ (Fig.

3.3.11). Aside from the quantitative disagreement, the qualitative agreement appears to be reasonable.

Both calculated and measured resolution profiles show slight asymmetry in the profiles. Small asymmetry is primarily due to the $\alpha$-particle range being nearly identical to the width of the detector whereas large asymmetry is seen for a detector with the thickness much larger than the range of $\alpha$-particles (Fig. 3.3.9). The poor calculated 


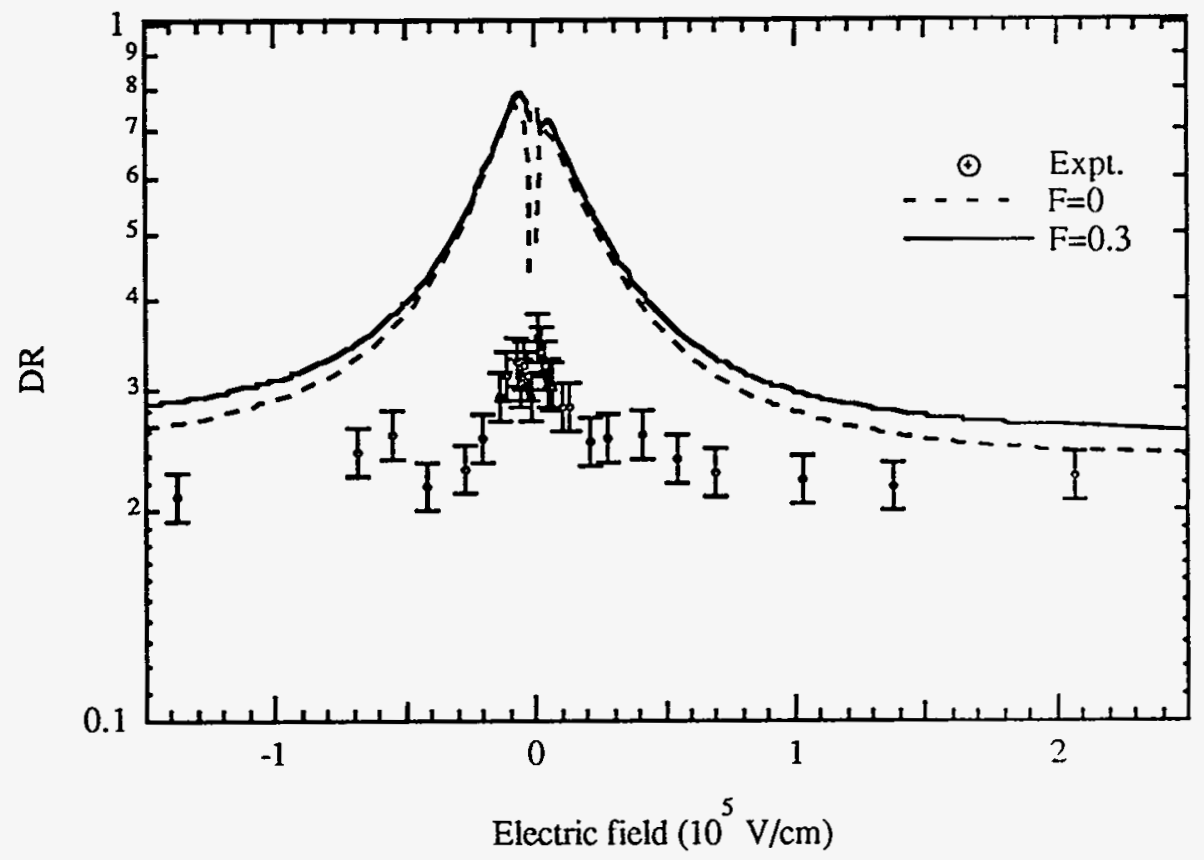

Figure 3.3.10: Comparison between calculated and experimental fractional resolution for detector SYM14m with $F=0,0.3$. The carrier mobilities and lifetimes were taken from Table 3.2.1. 


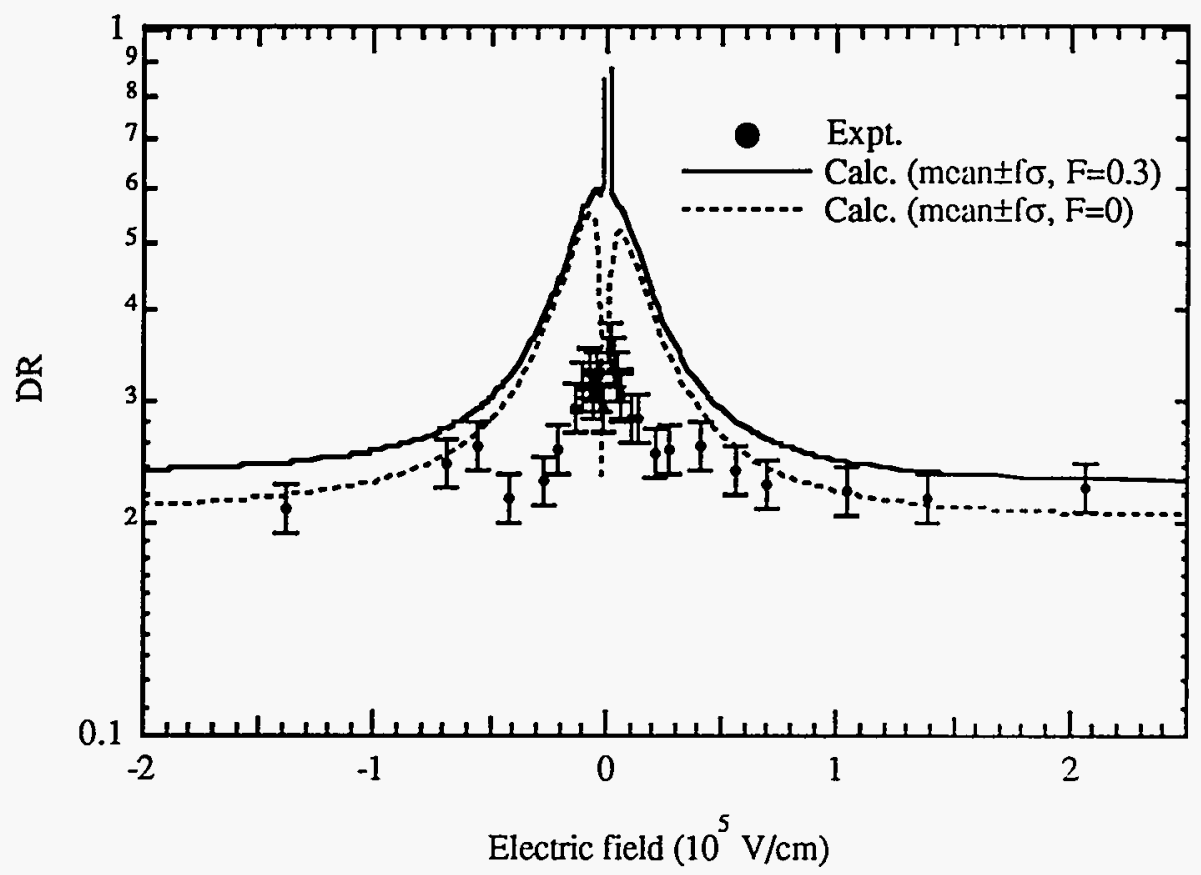

Figure 3.3.11: Comparison between calculation and experiment for detector SYM14m with $F=0,0.3$. The calculated profiles were obtained by modifying mean values of carrier mobilities and lifetimes from Table 3.2.1 by the quantity f $\sigma$.

resolution is attributed to a large disparity between the electron and hole transport properties in comparison to the carrier properties of SYM25m. It is not clear, however, why the calculated values are much larger than the measured values. But it is clear that large incomplete charge collection due to the small carrier mean free paths, i.e., large trap densities, is one of the primary causes for poor detector resolution.

In Fig. 3.3.12, the comparison between model and experiment for SYM70m is shown again with the Fano factor of 0.3. For this detector, the calculated fractional resolution fits well with the mean carrier transport properties for the entire range of electric fields studied. Again, by modifying the mean by fraction $f$ between $\pm(0.092$ 0.80 ) of the standard deviation, the agreement between model and experiment is only slightly improved. 
It is clear that the agreement between calculated and measured fractional resolution is qualitatively and quantitatively reasonable except for SYM14m when the mean transport properties were varied within $1 \sigma$. One of the main reasons for needing to vary the transport properties by fractions $\mathrm{f}$ may lie in the assumption that the carrier lifetime is constant as a function of applied field. It is apparent from Equ. 3.3.13 that the variance for the detector resolution is extremely sensitive to the carrier mean free paths. Therefore, if there was any field dependence in the carrier lifetimes, the fractional resolution may vary greatly as a function of applied field. Thus, in using the fractional resolution to estimate the mean carrier transport properties may not be feasible until a better understanding of the carrier lifetimes can be obtained.

As mentioned in Sec. 3.2 it is generally believed that natural diamond is very inhomogenious. The effect of material inhomogeneity would be to increase the fractional resolution. It appears that the material inhomogeneities cannot play a critical role in determining the detector resolution of the detectors studied here. 


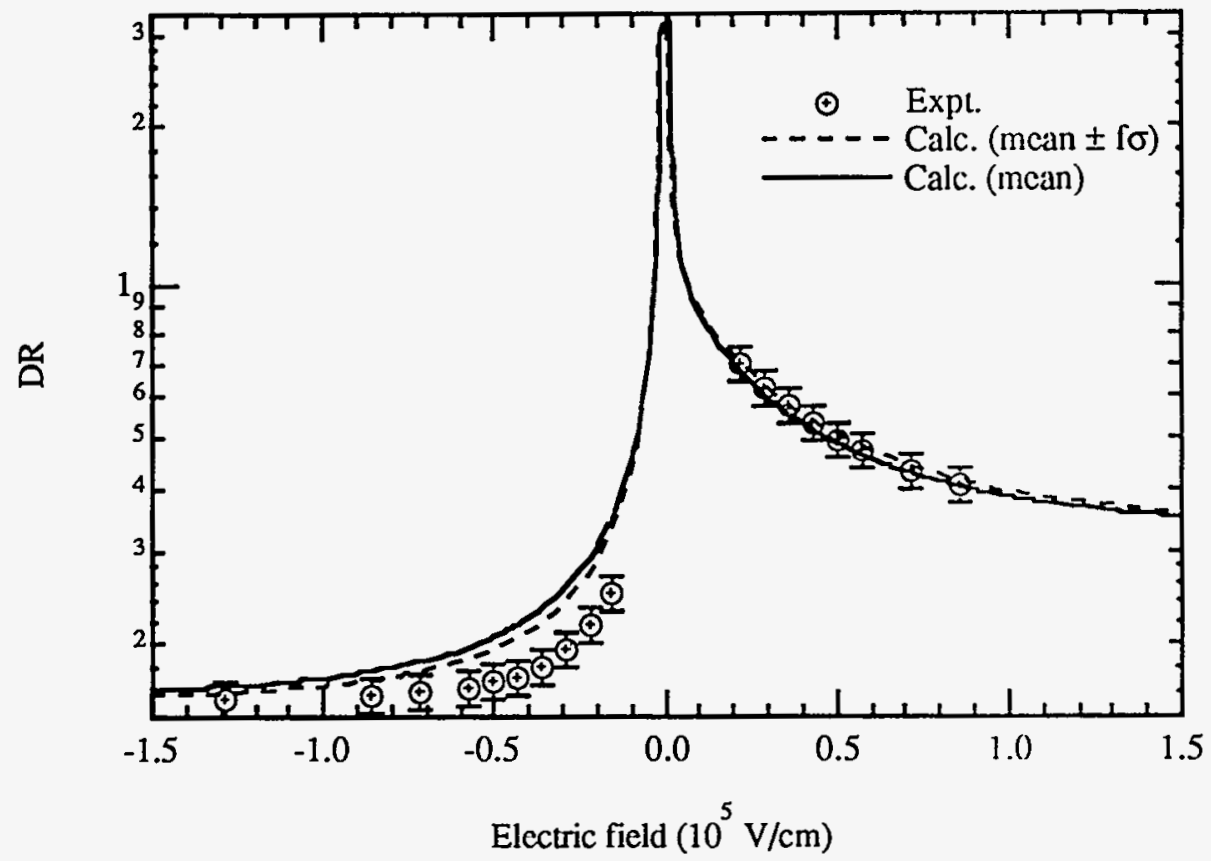

Figure 3.3.12: Comparison between calculated and experimental fractional resolution for detector SYM70m. ( ------- ) calculated width mean values of carrier mobilities and lifetimes from Table 3.2.1. ( $ـ$ ) calculated by modifying the mean by the quantity fo where the $f$ are given in Table 3.3.1. The Fano factor was taken to be 0.3 .

Table 3.3.1: List of the fractions, f, used to modify the mean values of $\mu_{c}, \tau_{c}, \mu_{h}$, and $\tau_{\mathrm{h}}$ for calculating fractional resolutions. The Fano factor was taken to be 0.3 .

\begin{tabular}{|ccccc|}
\hline Detector & $\pm f\left(\mu_{\mathrm{e}}\right)$ & $\pm \mathrm{f}\left(\mu_{\mathrm{h}}\right)$ & $\pm \mathrm{f}\left(\tau_{\mathrm{e}}\right)$ & $\pm \mathrm{f}\left(\tau_{\mathrm{h}}\right)$ \\
\hline SYM14m & $+9.3 \times 10^{-1}$ & $-7.7 \times 10^{-2}$ & $+7.8 \times 10^{-1}$ & $-5.9 \times 10^{-1}$ \\
SYM25m & $+7.9 \times 10^{-1}$ & $+9.5 \times 10^{-1}$ & $+9.8 \times 10^{-1}$ & $-6.3 \times 10^{-1}$ \\
SYM70m & $-9.2 \times 10^{-2}$ & $+8.0 \times 10^{-1}$ & $+2.3 \times 10^{-1}$ & $+7.3 \times 10^{-1}$ \\
\hline
\end{tabular}


References:

[1] K. Zanio, NIM, 83 (1970) 288

[2] K. Hecht, Z. Physik, 77 (1932) 235

[3] N. F. Mott and R. W. Gurney, Electronic Processes in Ionic Crystals, second ed., Oxford Press (1953) 122

[4] V. B. Gudden and R. Pohl, Z. Physik, 17 (1923) 170

[5] S. F. Kozlov, E. Belcarz, M. Hage-Ali, R. Stuck, P. Sieffert, NIM, 117 (1974) 277

[6] S. Ramo, P.I.R.E. 27 (1939) 584.

[7] See for examples, E. H. Putley, in Semiconductors and Semimetals, Vol. 12, Ed. R. K. Willardson and A. C. Beer, Academic Press, New York (1977) 143; D. H. Auston, in Ultrashort Laser Pulses and Applications, Ed. W. Kaiser, SpringerVerlag, Berlin (1988) 183; R. H. Bube, Photoconductivity of Solids, Rober Krieger, New York, (1978)

[8] R. Trammell and F. J. Walter, NIM, 76 (1969) 317

[9] T. A. McMath and M. Martini, NIM, 86 (1969) 245

[10] See for example, K. W. Böer, Survey of Semiconductor Physics, van Norstrand Reinhold, Ch 33 (1990)

[11] G. Davis, "Cathodoluminescence", The Properties of Diamond, ed. J. E. Field, Academic Press, 1979, p171

[12] L. S. Pan, Ph. D Thesis, Stanford University, 1991.

[13] H. J. McSkirmin, P. Andreatch, Jr. and P. Glynn, J. Appl. Phys., 43(3) (1982) 985

[14] F. Nava, C. Canali, C. Jacoboni and L Reggiani, Sol. State Comm, 33 (1980) 475

[15] L. Reggiani, S. Bosi, C. Canali and F. Nava, Sol. State Comm., 30 (1979) 333

[16] M. Martini, J. W. Mayer and K. R. Zanio, Applied Solid State Science: Advances in Materials and Device Research. Edited by R. Wolf, Vol. 3, Academic Press, New York, (1972) 181

[17] A. G. Redfield, Phys. Rev., 94(3) (1954) 526 
[18] E. A. Konorova and S. A. Shevchenko, Sov. Phys. - Semicond., 1(3) (1967) 299

[19] V. A. Chuenkov, Kratkie Soobshcheniya Pufizkika, 8 (1984) 25

[20] S. F. Kozlov, E. Belcarz, M. Hage-Ali, R. Stuck, P. Sieffert, NIM, 117 (1974) 277

[21] C. Canali, E. Gatti, S. F. Kozlov, P. F. Manfredi, C. Mangredotti, F. Nava and A. Quirini, NIM, 160 (1979) 73

[22] P. J. Dean and J. C. Male, J. Phys. Chem. Solids, 25 (1964) 311

[23] P. J. Kennedy, P.Royal Soc., London, A. Mat., 253 (1959) 37

[24] S. F. Kozlov, R. Stuck, M. Hage-Ali and P. Sieffert, IEEE Trans. Nucl. Sci., NS22 (1975) 160

[25] E. A. Konorova and S. F. Kozlov, Sov. Phys. - Semicond., 4 (1971) 160

[26] P. J. Dean, Phys. Rev. A, 139 (1965) 588

[27] J. F. Prins, Matl. Sci. Rep., 7(79, 80) (1992) 271

[28] R.R. Urlau, the late H. J. Logie and F. R. N. Nabarro, Proc. Phys. Soc. (London), 78 (1961) 256

[29] R. H. Bube, Photoelectronic Properties of Semiconductors, Cambridge University Press, Cambridge, (1992) 113

[30] L. S. Pan, Private communication

[31] C. A. Klein, J. Appl. Phys., 39 (1968) 2029

[32] P. A. Tove, Sens. and Act., 5 (1984) 103

[33] See for example, G. F. Knoll, Radiation Detection and Measurement, John Wiley \& Sons, Ch. 3 (1979); W. R. Leo, Techniques for Nuclear and Particle Physics Experiments: A How-to Approach, Springer-Verlag, Berlin (1987)

[34] U. Fano, Phys. Rev., 72 (1947) 26.

[35] G. Restelli and A. Rota, in Semiconductor Detectors, Edited by G. Bertolini and A. Coche, Elsevier-North Holland, Amsterdam (1968) 97

[36] G. D. Alkhazov, A. P. Komar and A. A. Vorobev, NIM, 48 (1967) 1. 
[37] G. Dearnaley and D. C. Northrop, Semiconductor Counters for Nuclear Radiation. John Wiley \& Sons, New York (1966) 91

[38] R. B. Day, G. Dearnaley and J. M. Palms, IEEE Trans. Nucl. Sci., NS-14(1) (1967) 487 


\section{Chapter 4: Time-resolved current experiments}

As mentioned in Chapter 2, time-resolved current measurements were performed to examine the dynamics of the charge collection process in RIC detectors. Because energy deposition is highly localized in the vicinity of the trajectory of a heavy charged particle, i.e., a proton, or the nucleus of a heavier element, significantly different response can be expected compared to the response from the uniform excitation normally encountered with photoconductors.

Given the understanding of diamond RIC detectors gained from the integratedcharge experiments, and especially the definition of the character of the electrical contacts, a model was developed to calculate the transient response of a detector under a variety of conditions. The model is represented in a one-dimensional transport code with spatially- and temporally-dependent variables. It is built around a transport code previously developed by Iverson [1].

In this chapter experimental results on the transient behavior of the detectors to both $\mathrm{He}$ and $\mathrm{Si}$ ions are described and compared to the response calculated with the model.

\subsection{Experimental results}

\subsubsection{Experimental conditions}

All experiments were performed with the system and in the manner described in Sec. 2.3. Before sampling transient pulses from the interaction of $\mathrm{He}$ or $\mathrm{Si}$ ions, bias voltage was applied to a detector and an average waveform from noise was developed by random sampling of the input voltage when the oscilloscope was allowed to trigger on system noise. This waveform was stored and automatically subtracted during development of the average waveform characteristic of stopping of a heavy ion. Table 
4.1.1 lists the experimental conditions under which the transient measurements were performed for the two ions studied. For all measurements, waveforms were first developed with positive polarity and then developed with negative polarity.

Table 4.1.1: Experimental conditions used for measurement of current transients. In order, the columns represent the ion type, its charge state, the ion kinetic energy $\left(\mathrm{E}_{\mathrm{I}}\right)$, the beam current $\left(\mathrm{I}_{\text {beam }}\right)$, the scattering angle $(\Theta)$ used to control the beam intensity on the target, and the distance $\left(R_{f c}\right)$ between the scattering foil and the collimator.

\begin{tabular}{|cccccc|}
\hline Ion type & $\begin{array}{c}\text { Ion charge } \\
\text { state }\end{array}$ & $\begin{array}{c}\mathrm{E}_{\mathrm{I}} \\
(\mathrm{MeV})\end{array}$ & $\begin{array}{c}\mathrm{I}_{\text {beam }} \\
(\mathrm{nA})\end{array}$ & $\begin{array}{c}\Theta \\
(\mathrm{deg})\end{array}$ & $\begin{array}{c}\mathrm{R}_{\mathrm{fc}} \\
(\mathrm{cm})\end{array}$ \\
\hline $\mathrm{He}$ & $1+$ & 5 & 70 & 2.7 & 20 \\
$\mathrm{Si}$ & $3+$ & 10 & 25 & 2.7 & 20 \\
\hline
\end{tabular}

4.1.2 Results from the interaction of 5-MeV He+ ions with the detector HE6.4

Fig 4.1.1 shows average current transients from the interaction of $5-\mathrm{MeV} \mathrm{He}^{+}$ions in detector HE6.4 as a function of applied bias voltage in the range $\pm(75-200) \mathrm{V}$, corresponding to applied electric fields in the range $\pm(0.37-1.0) \times 10^{5} \mathrm{~V} / \mathrm{cm}$. The amplitudes of all waveforms are given relative to the peak voltages found experimentally. In all cases, the main pulse is followed by a small transient beginning about 250 ps later. This represents the time required for an electromagnetic disturbance to propagate a distance of $2.4 \mathrm{~cm}$ through the system, and thus the transient can be identified as a reflection of the original current transient at the boundary between the mid- and the endsections of the detector package (Fig. 2.3.1-A). The fact that the ratio of the amplitudes of the two peaks is roughly independent of the applied bias is consistent with this 
A)

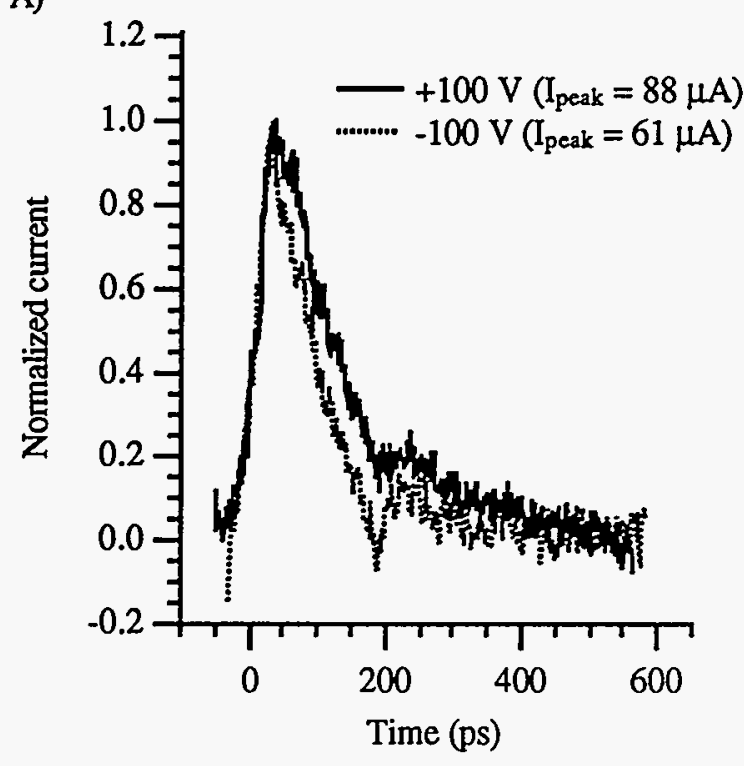

C)

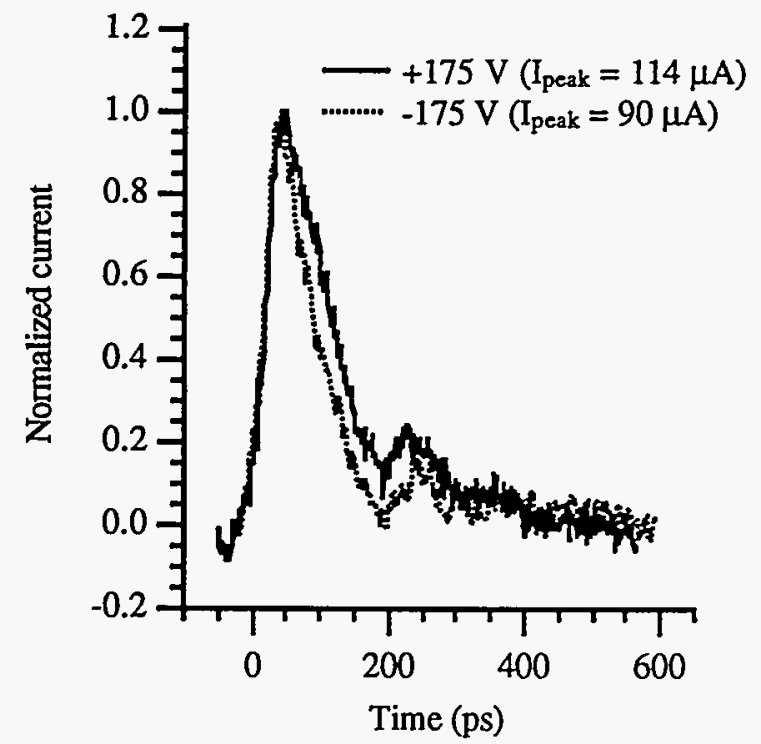

B)

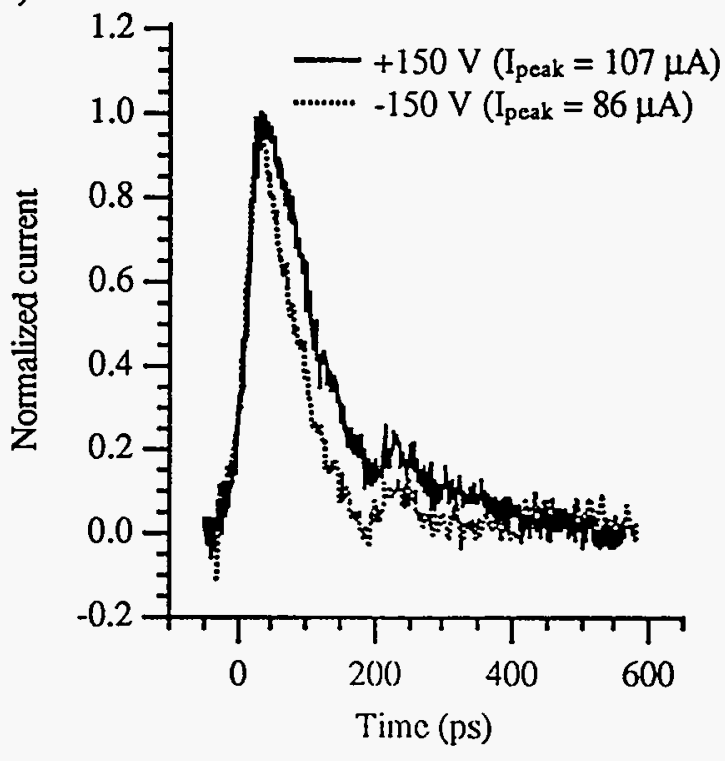

D)

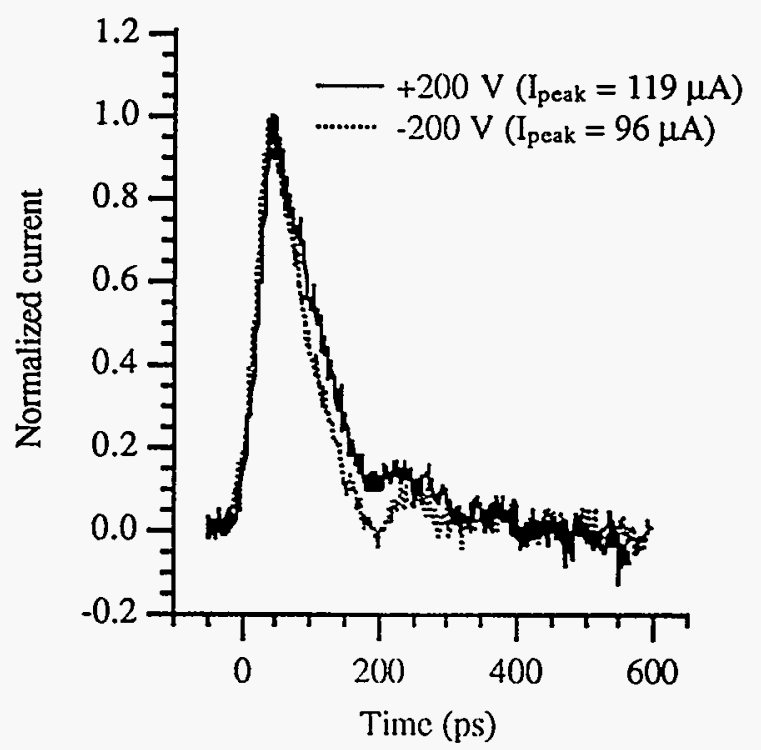

Figure 4.1.1: Average time-resolved current pulses from detector system HE6.4 excited with 5-MeV He ions. 
interpretation. Due to the limited sensitivities of the recording system and the detector, no transient was observed below a bias voltage of $75 \mathrm{~V}$.

The time variation of the transient from interaction of individual ions in the detector is roughly Gaussian rather than exponential in form. This may be taken as further evidence for the non-injecting character of the contacts. A strictly exponential waveform would be expected if the contacts were injecting because the time dependence of the current would then be a function of carrier lifetime only.

In table 4.1.1 are listed the relevant parameters for each of the measured current transients from the interaction of $5 \mathrm{MeV}$ He ions in detector HE6.4, including the magnitude of the applied electric field, the peak current (Ipeak) of the transient, rise- and fall-times $\left(T_{\text {rise }}, T_{\text {fall }}\right.$, the FWHM of the pulse, the total charge contained in the pulse $\left(Q_{\text {coll }}\right)$, the corresponding charge collection efficiency $(\eta)$, and the detector sensitivity (S) , defined as the charge collected per unit energy deposited per unit applied electric field. Rise- and fall times are defined as the time interval for a transient to vary from $10 \%$ to 90 $\%$ of the peak height on the leading and trailing edges of a pulse, respectively. The magnitudes of these parameters must be considered approximate because they can be quite sensitive to the estimated baseline current. A better measure of a characteristic time for the transients would be the FWHM which is normally less sensitive to the nature of the tails of a pulse. The charge collection efficiency is defined as the ratio of the collected charge, $Q_{\text {coll }}$, to the total charge created by stopping of the He ion in the detector.

The FWHM of the transients reported in Table 4.1.2 are roughly independent of the applied field or tend to decrease slightly with increase in the applied field. In either case, their magnitudes are about what is expected from the saturation velocities that can be estimated from the mobilities found in the charge integrated experiments. For example, if the carrier velocity is taken as its saturation value of $2 \times 10^{7} \mathrm{~cm} / \mathrm{sec}$, the sum of the average transit-times of the two carrier types across the detector is about 100 ps. 
With respect to the magnitudes of the measured rise times, it is readily demonstrated that they cannot represent the charge production rate in the detector. In the ideal case, where the recording system introduced no perturbation on the waveform, the rise time of a pulse should be a function only of the rate of charge production during slowing down of a heavy ion. With $5-\mathrm{MeV} \mathrm{He}^{+}$ions, the average stopping time calculated with Equ. 2.2.1, and assuming using a mean ion range of $12 \mu \mathrm{m}$ (Fig. 4.1.2) is about 1.3 ps. Because the response time of the recording system is about an order of magnitude larger, the time required for charge production can be considered to be instantaneous. Therefore, observed rise times with magnitudes larger than the system rise time cannot be characteristic of the charge production. In the present case, where very high initial charge densities are involved, the rise times are generally expected to be characteristic of the screening of the applied field by the electron-hole plasma produced by the heavy ion. Thus, the measured rise times can be taken as direct measures of the plasma time.

While the errors are large, the mean rise times given in Table 4.1.2 appear to decrease somewhat as the applied field is increased. Qualitatively, this is consistent with the expectation of greater penetration of high fields into a plasma and the subsequent increase in the rate of plasma erosion by transport of charge away from the site of formation. A small dependence of rise time on applied field was also seen in previous measurements of ultrafast current transients in Si and GaAs diodes 12, 3]. 


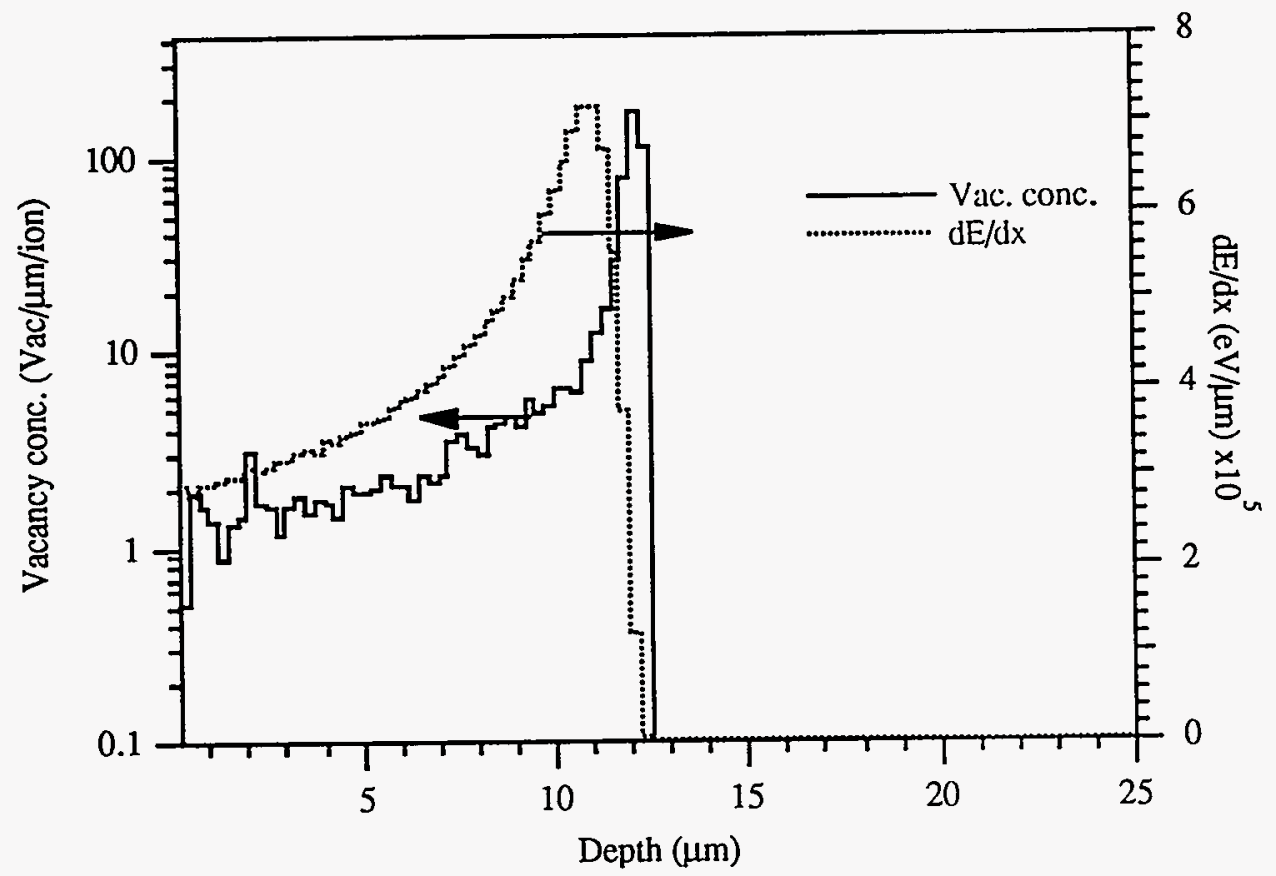

Figure 4.1.2: Stopping power and ion-induced vacancy concentration distributions for $5-\mathrm{MeV} \mathrm{He} \mathrm{He}^{+}$-ion as calculated with the code TRIM [4]. The vacancy concentration is in units of vacancies per incident ion per micron traversed by the $\mathrm{He}^{+}$ion. 
Table 4.1.2: Pulse parameters as a function of applied bias for detector HE6.4

\begin{tabular}{|cccccccc|}
\hline $\begin{array}{c}|\vec{E}| \\
(\mathrm{KV} / \mathrm{cm})\end{array}$ & $\begin{array}{c}\mathrm{I}_{\text {peak }} \\
(\mu \mathrm{A})\end{array}$ & $\begin{array}{c}\mathrm{FWHM} \\
(\mathrm{ps})\end{array}$ & $\begin{array}{c}\mathrm{T}_{\text {rise }} \\
(\mathrm{ps})\end{array}$ & $\begin{array}{c}\mathrm{T}_{\text {fall }} \\
(\mathrm{ps})\end{array}$ & $\begin{array}{c}\text { Qcoll } \\
(\mathrm{fC})\end{array}$ & $\eta$ & $\begin{array}{c}\mathrm{S} \\
(\mathrm{C} / \mathrm{J} / \mathrm{V}) \times 10^{-5}\end{array}$ \\
\hline 50 & $83 \pm 8.3$ & $113 \pm 11.3$ & $42 \pm 4.2$ & $185 \pm 18.5$ & $10.7 \pm 1.1$ & $0.19 \pm 0.02$ & $13.4 \pm 1.3$ \\
75 & $101 \pm 10.1$ & $103 \pm 10.3$ & $38 \pm 3.8$ & $179 \pm 17.9$ & $11.8 \pm 1.2$ & $0.21 \pm 0.02$ & $9.9 \pm 1.0$ \\
87.5 & $113 \pm 11.3$ & $96 \pm 9.6$ & $34 \pm 3.4$ & $137 \pm 13.7$ & $12.4 \pm 1.2$ & $0.22 \pm 0.02$ & $8.8 \pm 0.9$ \\
100 & $117 \pm 11.7$ & $95 \pm 9.5$ & $37 \pm 3.7$ & $193 \pm 19.3$ & $12.7 \pm 1.3$ & $0.22 \pm 0.02$ & $7.9 \pm 0.8$ \\
-50 & $59 \pm 5.9$ & $82 \pm 8.2$ & $40 \pm 4.0$ & $114 \pm 11.4$ & $6.1 \pm 0.6$ & $0.11 \pm 0.01$ & $7.6 \pm 0.8$ \\
-75 & $80 \pm 8.0$ & $78 \pm 7.8$ & $40 \pm 4.0$ & $104 \pm 10.4$ & $8.3 \pm 0.8$ & $0.15 \pm 0.02$ & $6.9 \pm 0.7$ \\
-87.5 & $89 \pm 8.9$ & $74 \pm 7.4$ & $36 \pm 3.6$ & $108 \pm 10.8$ & $8.9 \pm 0.9$ & $0.16 \pm 0.02$ & $6.3 \pm 0.6$ \\
-100 & $90 \pm 9.0$ & $78 \pm 7.8$ & $38 \pm 3.8$ & $100 \pm 10.0$ & $8.8 \pm 0.9$ & $0.15 \pm 0.02$ & $5.5 \pm 0.6$ \\
\hline
\end{tabular}

The data in Table 4.1.2 indicate that the charge collection efficiency with negative polarity is about $2 / 3$ of that found with positive polarity. If, in fact, the characteristics of the contacts were identical, i.e., they were non-injecting to both electrons and holes, the response of the detector should be independent of the polarity of the applied field. Given the experimental results discussed in Chap. 3 , there is no indication for injecting characteristics of the contacts and some other factor must give rise to this difference in ion collection efficiencies. Based on experience with a high intensity beam on target, and considering that the set of data from positive polarities was taken before that of negative polarity, a likely source for the discrepancy is radiation damage from the ion bombardment itself.

From Table 4.1.1, the rate at which the $\mathrm{He}^{+}$ions interact with the detector is about $7.3 \times 10^{3} \mathrm{sec}^{-1}$. The entire set of data on current transients with positive polarity, was taken first over a total irradiation period of $35 \mathrm{~min}$. , and the total $\mathrm{He}^{+}$ion fluence over this period is then estimated to be about $6 \times 10^{12} \mathrm{~cm}^{-2}$. From the vacancy production 
profile shown in Fig. 4.1.2, we can expect a vacancy density to vary in the range $1 \times 10^{12}$ $1 \times 10^{16} \mathrm{~cm}^{-3}$ over the mean trajectory of the $\mathrm{He}^{+}$ions in the detector. Such defects are likely to represent additional carrier trapping/recombination sites. As a result, the mean carrier lifetime should be reduced, the response rate of the detector after the damage should be increased, and the total charge collected should be reduced relative to the case of an undamaged detector. In fact, the characteristics of the data taken with negative polarity display, qualitatively, just the characteristics expected from these effects. In addition to the reduction in collected charge, the FWHM of the negative polarity transients are also reduced.

In Fig. 4.1.3 the sensitivity, $S$, is shown as a function of the absolute value of applied electric field for the two sets of measurements. Within experimental errors, the variation in sensitivity with applied field is identical for both polarities. This is completely consistent with the assumption that radiation-induced defects are the principal source for the differences in sensitivities of the two sets of measurements. 


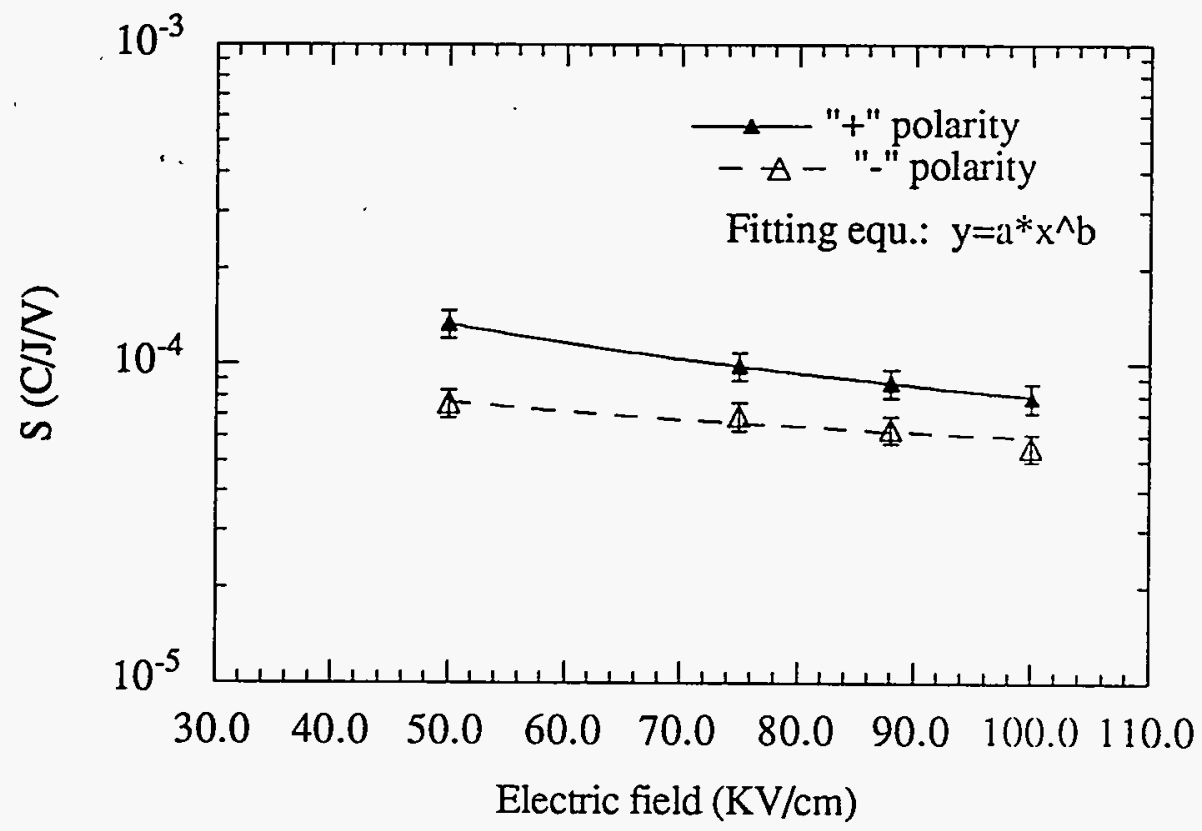

Figure 4.1.3: Sensitivity, $\mathrm{S}$, as a function of the absolute value of the applied electric field. The lines drawn through the data represent power fits of the form $y=a x^{b}$ :

'+' polarity: $\mathrm{a}=(253.8 \pm 7.6) \times 10^{-5} \mathrm{C} / \mathrm{J} / \mathrm{V} ; \mathrm{b}=(-75.2 \pm 0.7) \times 10^{-2}$

'-' polarity: $\mathrm{a}=(333.2 \pm 11.7) \times 10^{-5} \mathrm{C} / \mathrm{J} / \mathrm{V} ; \mathrm{b}=(-37.5 \pm 8.5) \times 10^{-2}$

4.1.3 Results from the interaction of 10-MeV Si3+ ions with the detector SI6.9

In Figures 4.1.5 and 4.1.6 are shown the current transients measured when the detector SI6.9 was excited with $10-\mathrm{MeV} \mathrm{Si}^{3+}$ ions and the bias voltage was varied in the range $\pm(75-200) \mathrm{V}$ (electric fields in the range $\pm\left(0.37-1.0 \times 10^{5}\right) \mathrm{V} / \mathrm{cm}$. Again, all waveforms have been normalized relative to their peak amplitudes. The second peaks evident in the figures are due to the same type of reflection as discussed above for the measurements with $\mathrm{He}^{+}$ions. The average stopping time for $10-\mathrm{MeV} \mathrm{Si}^{3+}$ ions calculated with Equ. 2.3.1, and using the mean ion range of $2.6 \mu \mathrm{m}$ shown in Fig. 4.1.2. is about 0.5 ps. Once again, this is clearly much shorter than the rise time of the recording system and the excitation can be considered an impulse. The current transients 
A)

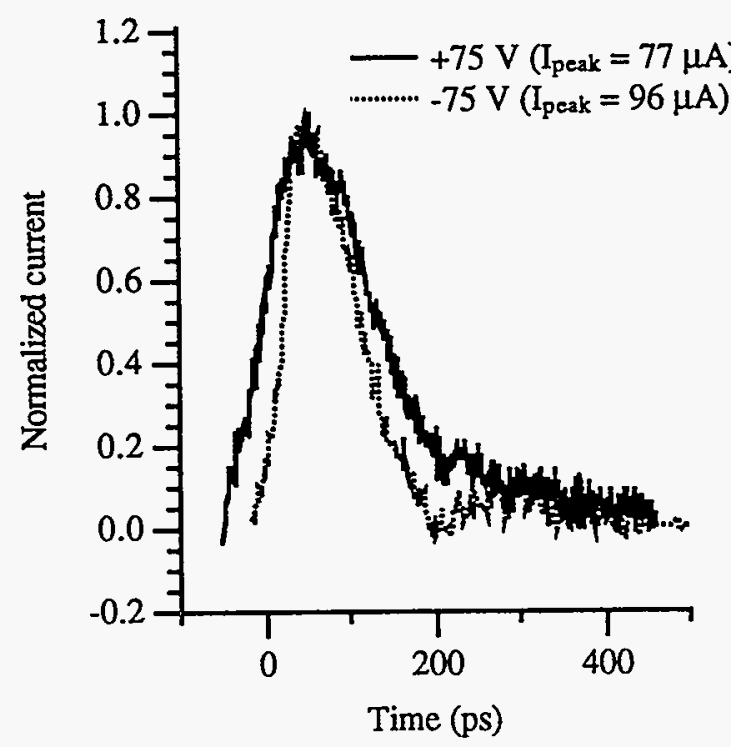

C)

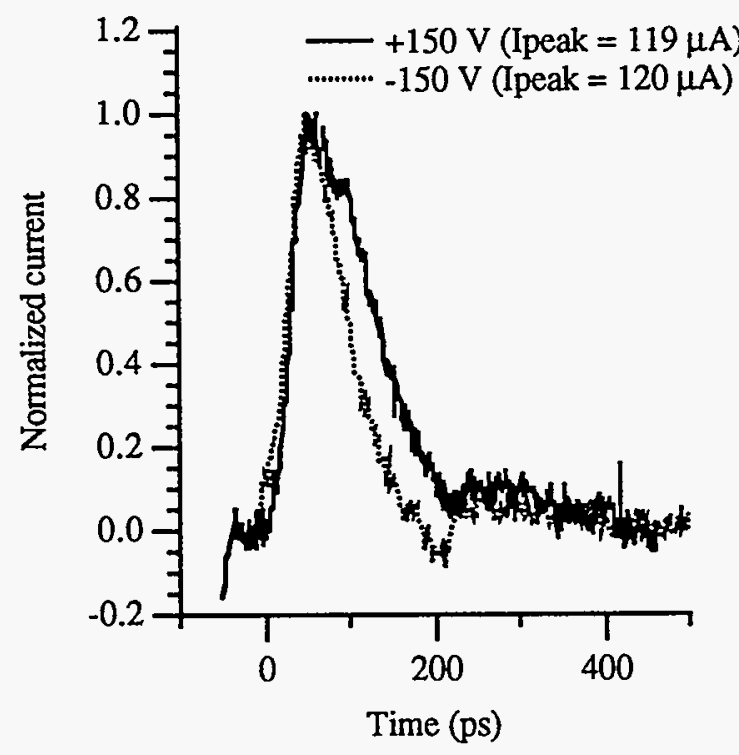

B)

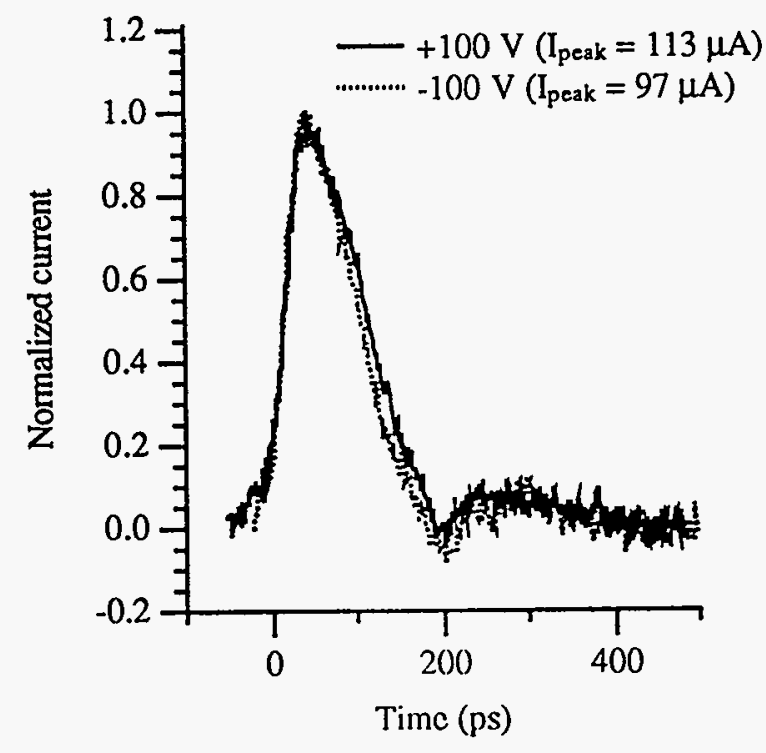

D)

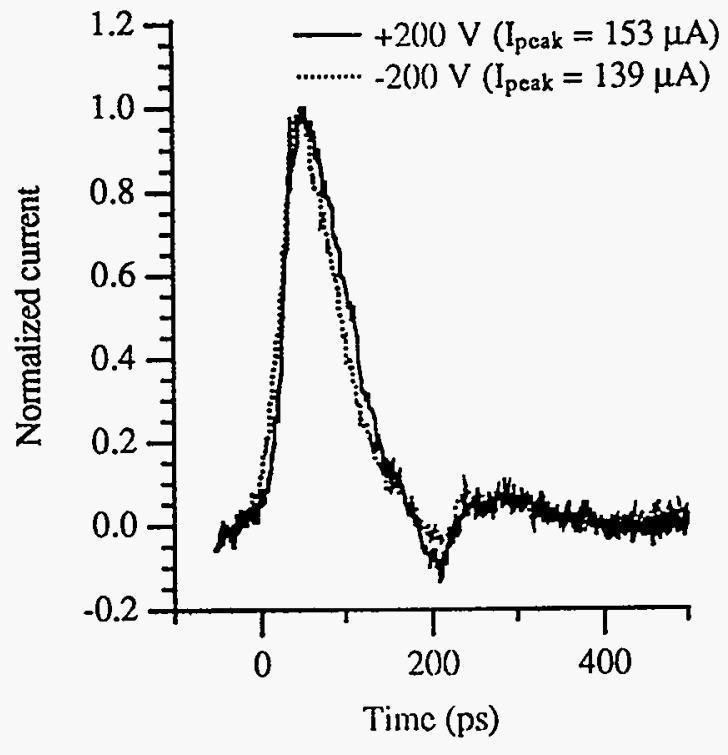

Figure 4.1.4: Mean time-resolved current transients from detector SI6.9 excited with $10-\mathrm{MeV} \mathrm{Si}^{3+}$ ions. 
found with negative polarity are always significantly narrower than those found with positive polarity. All of the waveforms are quasi- Gaussian rather than exponential in form.

A listing of the transient characteristics is given in Table 4.1.3. As in the case of the experiments with $\mathrm{He}^{+}$ions, all measurements with positive polarity were taken before measurements with negative polarity. According to the experimental conditions given in

Sec. 4.1.1, the interaction rate of the ions with the detector was about $9 \times 10^{3} \mathrm{~s}^{-1}$, and the total fluence of $\mathrm{Si}^{+3}$ ions over the duration of these measurements (about $20 \mathrm{~min}$.) is then roughly $3 \times 10^{12} \mathrm{~cm}^{-2}$. From Fig 4.1 .5 , this fluence would lead to an induced vacancy concentration in the range of about $10^{17}-2 \times 10^{20} \mathrm{~cm}^{-3}$ over the mean trajectory of an ion. Neglecting annealing during ion bombardment, this range is comparable or greater than the nominal nitrogen concentration in type Ila diamond. Consequently, the effect of the additional defects on carrier lifetimes is expected to be large. Indeed, essentially all of the transient characteristics given in Table 4.1.3 are smaller for measurements taken with negative polarity. In particular, both the FWHM and the total collected charge at the same field magnitude are significantly smaller in the case of negative polarity.

Because the excitation density is expected to be considerably larger by virtue of a shorter range and larger total energy deposition, the plasma time, and hence pulse rise times, are expected to be larger for the case of the $\mathrm{Si}$ ion interactions as compared to the i $\mathrm{MeV}$ He ions discussed above. However, reference to Tables 4.1.2 and 4.1.3 indicate that for the same electric field, the rise times from stopping of the $\mathrm{Si}$ ions are approximately equal to those for the stopping of $\mathrm{He}^{+}$. Because the plasma time is dependent on the charge transport properties [4], this result may reflect differences in the materials properties of the two detectors used for the measurements. Nevertheless, the rise time of the transients are seen to decrease with increasing bias voltage in agreement with the higher plasma erosion rate expected. This observation is consistent with previous reports 


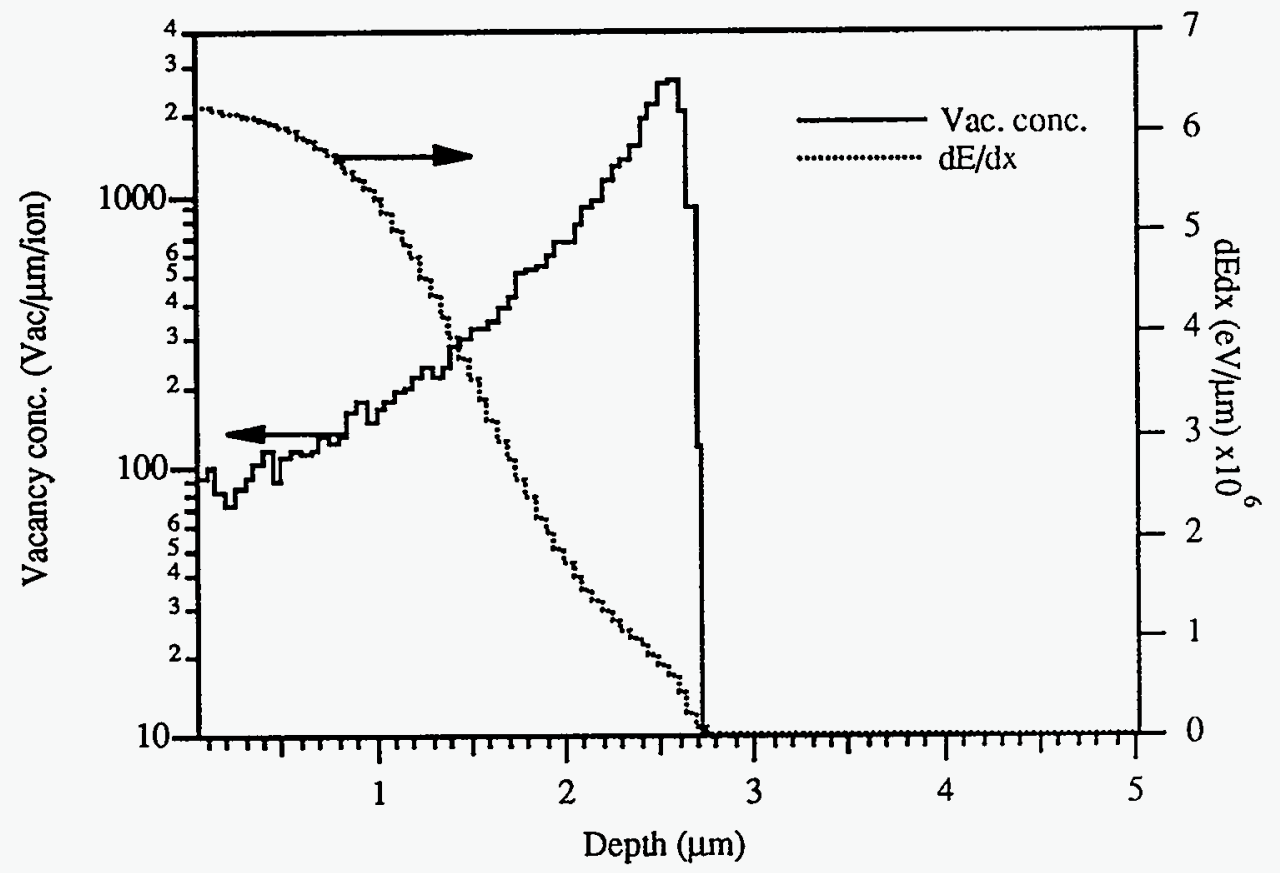

Figure 4.1.5: Stopping power and vacancy concentrations for the interaction of $10 \mathrm{MeV}$ Si ions in diamond as calculated with TRIM [2].

Table 4.1.3: Pulse information as a function of bias voltage for detector SI6.9.

\begin{tabular}{|cccccccc|}
\hline $\begin{array}{c}|\vec{E}| \\
(\mathrm{KV} / \mathrm{cm})\end{array}$ & $\begin{array}{c}\mathrm{I}_{\text {peak }} \\
(\mu \mathrm{A})\end{array}$ & $\begin{array}{c}\text { FWHM } \\
(\mathrm{ps})\end{array}$ & $\begin{array}{c}\mathrm{T}_{\text {rise }} \\
(\mathrm{ps})\end{array}$ & $\begin{array}{c}\mathrm{T}_{\text {fall }} \\
(\mathrm{ps})\end{array}$ & $\begin{array}{c}\mathrm{Q}_{\text {coll }} \\
(\mathrm{fC})\end{array}$ & $\begin{array}{c}\eta \\
(\mathrm{C} / \mathrm{J} / \mathrm{V}) \times 10^{-5}\end{array}$ \\
\hline 37.5 & $69 \pm 7$ & $149 \pm 15$ & $67 \pm 7$ & $151 \pm 15$ & $12.6 \pm 1.3$ & $0.11 \pm 0.01$ & $10.4 \pm 1.0$ \\
50 & $109 \pm 11$ & $100 \pm 10$ & $41 \pm 4$ & $107 \pm 11$ & $12.8 \pm 1.3$ & $0.11 \pm 0.01$ & $8.2 \pm 0.8$ \\
75 & $98 \pm 10$ & $105 \pm 11$ & $29 \pm 3$ & $125 \pm 13$ & $12.3 \pm 1.2$ & $0.11 \pm 0.01$ & $5.4 \pm 0.5$ \\
100 & $153 \pm 15$ & $82 \pm 8$ & $35 \pm 4$ & $96 \pm 10$ & $13.2 \pm 1.3$ & $0.12 \pm 0.01$ & $4.2 \pm 0.4$ \\
-37.5 & $93 \pm 9$ & $90 \pm 9$ & $37 \pm 4$ & $104 \pm 10$ & $8.4 \pm 0.8$ & $0.07 \pm 0.01$ & $7.0 \pm 0.7$ \\
-50 & $96 \pm 10$ & $90 \pm 9$ & $35 \pm 4$ & $106 \pm 11$ & $9.2 \pm 0.9$ & $0.08 \pm 0.01$ & $5.9 \pm 0.6$ \\
-75 & $119 \pm 12$ & $77 \pm 8$ & $41 \pm 4$ & $86 \pm 9$ & $10.6 \pm 0.9$ & $0.09 \pm 0.01$ & $4.6 \pm 0.5$ \\
-100 & $139 \pm 14$ & $72 \pm 7$ & $41 \pm 4$ & $85 \pm 8$ & $11.7 \pm 1.0$ & $0.10 \pm 0.01$ & $3.3 \pm 0.4$ \\
\hline
\end{tabular}


describing modeling and experimental studies on plasma erosion and single-event upset from high-energy ion interactions $[3,5,6,7,8,9,10]$. In addition, the charge collection efficiency is seen to be about a factor of 2 less than found with the $\mathrm{He}^{+}$excitation. This is consistent with differences in plasma properties because plasma screening of the carriers is expected to be greater. At the highest electric field, the charge collection efficiency is about 0.1 .

In Fig. 4.1.6 the detector sensitivity is plotted as a function of applied electric field. In general, the sensitivities are smaller than observed with the $\mathrm{He}^{+}$ions, due predominantly to poorer charge collection efficiencies. Of particular interest is the fact that the sensitivity decreases more rapidly with increasing field than seen in the data from the $\mathrm{He}^{+}$experiments. Once again, this may be an indication of differences in carrier transport properties, and will be examined further in Sec. 4.3.

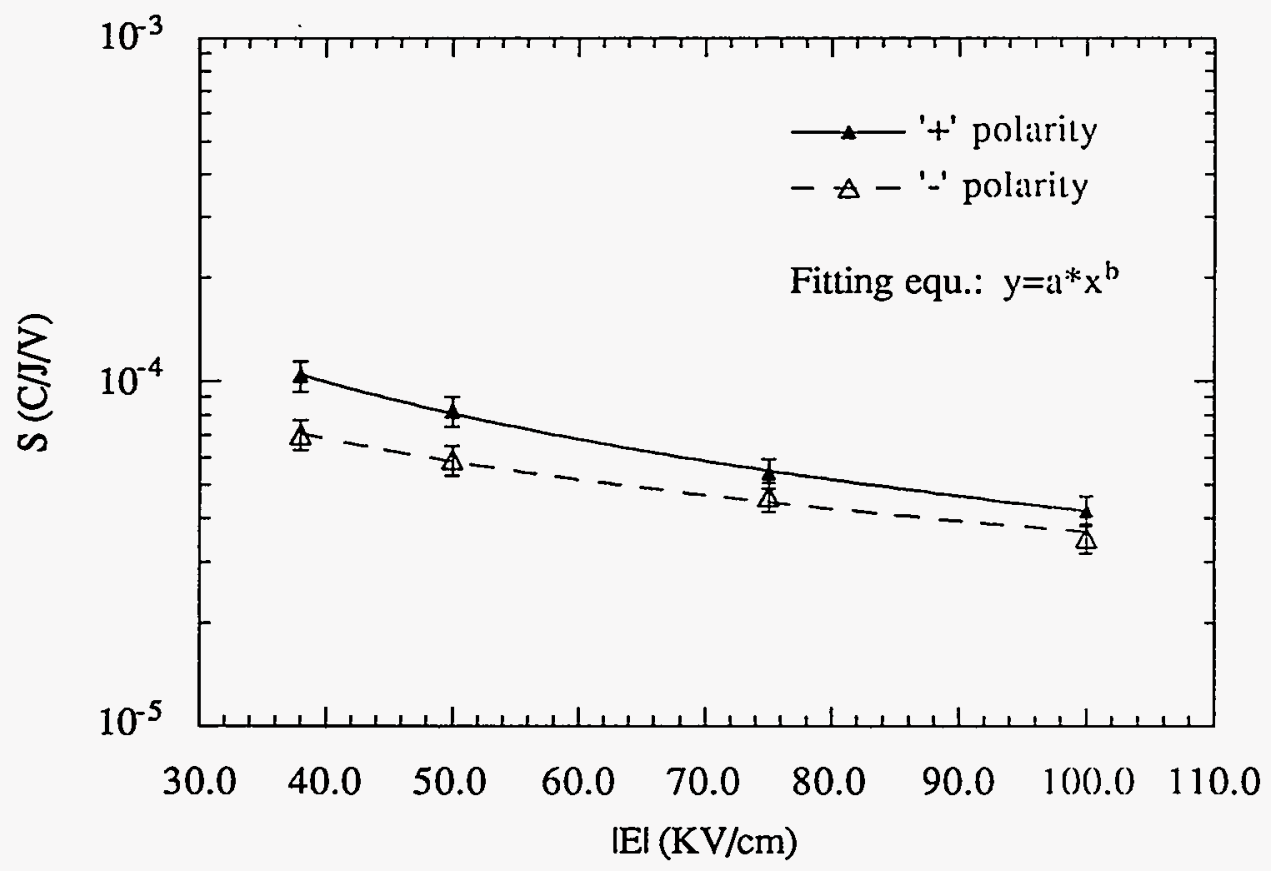

Figure 4.1.6: Sensitivity, $\mathrm{S}$, as a function of the absolute value of the applied electric field. The lines drawn through the data represent power fits of the form $y=a x^{b}$ :

' + ' polarity: $\mathrm{a}=(327.5 \pm 34.1) \times 10^{-5} \mathrm{C} / \mathrm{J} / \mathrm{V} ; \mathrm{b}=(-94.6 \pm 2.7) \times 10^{-2}$

$\therefore$ polarity: $\mathrm{a}=(83.7 \pm 14.8) \times 10^{-5} \mathrm{C} / \mathrm{J} / \mathrm{V} ; \mathrm{b}=(-68.0 \pm 4.5) \times 10^{-2}$ 


\subsection{Modeling of time-resolved current transients}

In many analyses of conductivity modulated devices given in standard semiconductor physics texts, such as the ones referenced in Sec. 1.4, the conditions considered are restricted to small deviations of total carrier densities from the (thermal) equilibrium densities. For example, the electron and the hole densities are usually written as

$$
\begin{aligned}
& \mathrm{n}=\mathrm{n}_{\mathrm{o}}+\delta \mathrm{n} \\
& \mathrm{p}=\mathrm{p}_{\mathrm{o}}+\delta \mathrm{p}
\end{aligned}
$$

where $\mathrm{n}=$ total conduction electron density

$$
\begin{aligned}
& \mathrm{p}=\text { total valence hole density } \\
& \mathrm{n}_{\mathrm{O}}=\text { thermal equilibrium conduction electron density } \\
& \mathrm{p}_{\mathrm{O}}=\text { thermal equilibrium valence hole density } \\
& \delta \mathrm{n}=\text { excess conduction electron density caused be external excitation } \\
& \delta \mathrm{p}=\text { excess valence hole density caused by external excitation }
\end{aligned}
$$

Such analyses are essentially perturbation calculations, and describe the behavior of $n(x, t)$ and $\mathrm{p}(\mathrm{x}, \mathrm{t})$ analytically when $\frac{\delta n}{n_{o}} \ll 1$ and $\frac{\delta p}{p_{o}} \ll 1$.

Adler, et al. [11], have analyzed a case where dp and dn are comparable to their thermal equilibrium values. However, they consider the response characteristics of a device only after the material has relaxed sufficiently that the small excess carrier generation approximation can be used.

In the late 80 's, Iverson, et al. [12 13] investigated the response of a photoconductor when the excess carrier density is large compared to eq̣uilibrium values, i.e., $\frac{\delta n}{n_{0}} \gg 1$ and $\frac{\delta p}{p_{o}} \gg 1$. Due to the nature of the partial differential equations involved, numerical methods were used to solve the set of continuity equations that were developed. The numerical reformulation of the continuity equations for semiconductors 
given by Gummel and Schrefetter [14] were used in their analysis. Rather than discretizing carrier densities, Iverson et al. discretized the current density equations, and the carrier densities were taken as manifestations of the current density equations. The authors have shown in Ref. 10 that with this approach, the problem of recurring numerical instabilities can be avoided when the potential between successive spatial points becomes greater than $(\mathrm{kT} / \mathrm{e})$. This approach is adopted in this thesis to solve the equations describing a one-dimensional model of the transient response of diamond detectors to excitation with heavy ions.

\subsubsection{Model assumptions}

In reality, the description of the ion interaction with the detector and the subsequent detector response should be treated in three dimensions (see the schematic diagram in Fig. 4.2.1-A). A possible set of carrier density distributions in all directions is shown in Fig. 4.2.1-B: The carrier distributions in $\mathrm{x}$-and $\mathrm{y}$-directions are assumed to be Gaussian in form whereas the carrier density distribution in the z-direction would be dictated by the ion stopping power distribution.

A simplifying assumption that should not lead to significant error is to take the stopping power of the heavy ion as a constant. In the limit that the ion track is long compared to the track's radius, the transport characteristics of the charges in any disc slice of thickness $\mathrm{dR}$ will be identical to all others. Then, to a fair approximation, the transport of ions can be studied by considering the 2-dimensional transport of the charge in a disc as indicated in Fig. 4.2.2.

Reduction of the two-dimensional transport problem to a one dimensional representation cannot be accomplished without significant loss in detail because of the large radial variation in charge density likely to be found in the real case of stopping of heavy ions. The carrier density near the edge of the disk is sufficiently smaller than near the center that space charge effects will be much less important to the transport. Per unit 
A)

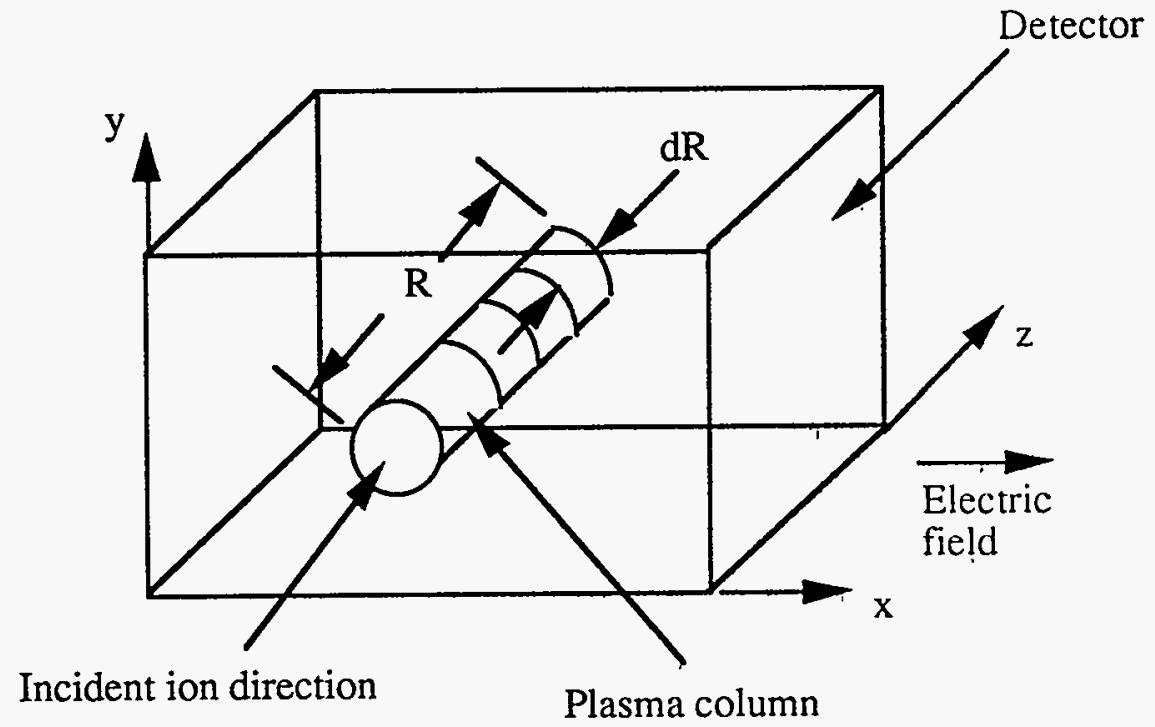

B)

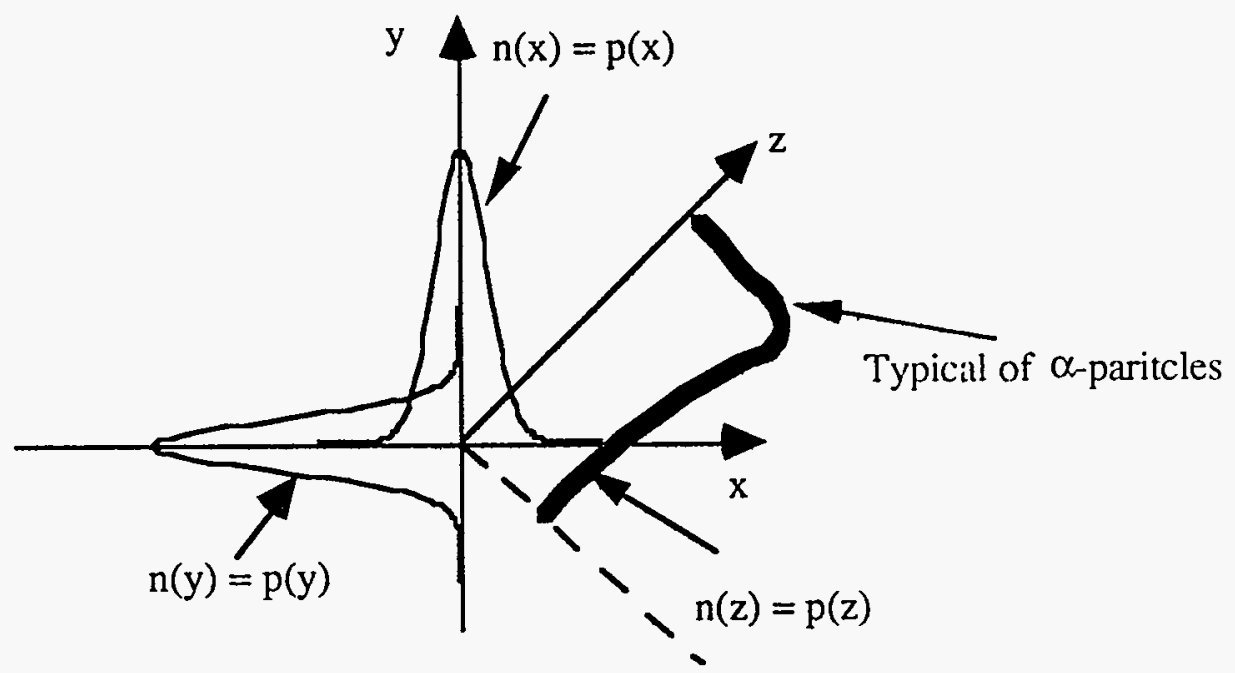

Figure 4.2.1: Schematic diagram of the electron-hole plasma created in the detector by stopping of a single incident ion.

A) Schematic of the envelope of the initial charge distribution. The dimension $\mathrm{R}$ is the mean range of the ion. The dimension $\mathrm{d} R$ represents the thickness of a small disc slice of the initial distribution. B) Schematic of the carrier density distributions along all three coordinates. The origin is located at the center of plasma at the surface of the detector. 
charge, charge collection from a slice of the disc of dimension dy located near the circumference of the initial distribution will be much higher than charge collection from a slice that includes the central portion of the distribution.

Nevertheless, the main features of the physics of charge collection as well as the overall magnitude of signals can be maintained with the following simplification; in place of the radially-symmetric charge distribution, the charge distribution will be approximated as a one-dimensional Gaussian as indicated in Fig. 4.2.3. This is equivalent to following the transport of ions present initially in a thin slice of the disc of Fig. 4.2.2-A parallel to the $\mathrm{x}$-axis and centered about its axis.

Quantitatively, this simplification should overestimate the effects due to space charge relative to the two-dimensional approximation. Any factors leading to incomplete charge collection should be emphasized, and all other things being equal, we expect to see model calculations underestimating the charge collected in comparison to any experimental results.

A schematic of the simplified model for charge collection in a one-dimensional detector is shown in Fig. 4.2.4. The mathematical formulation of its description was developed under the following set of assumptions:

1) Because the stopping times for $5-\mathrm{MeV} \mathrm{He}^{+}$and $10-\mathrm{MeV} \mathrm{Si}^{3+}$ are less than about $1.3 \mathrm{ps}$, all free carriers generated from the interaction of ions with the detector are assumed to be produced instantaneously, i.e., no relaxation of the detector occurs during the slowing down of an ion.

2) Bias voltage applied externally is considered to drop entirely across the detector and is assumed to be constant throughout a current transient. This is justified because the resistivity of the detector is greater than $10^{10} \Omega$-cm, much greater than the resistivity of the electrical contact of approximately $10^{-5} \Omega-\mathrm{cm}^{-2}[15,16]$. 
A)

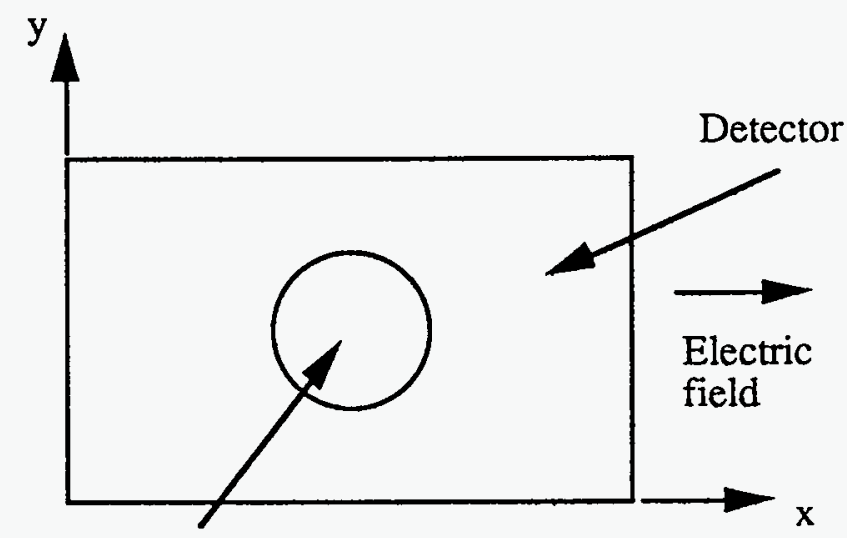

2-D plama disk

B)

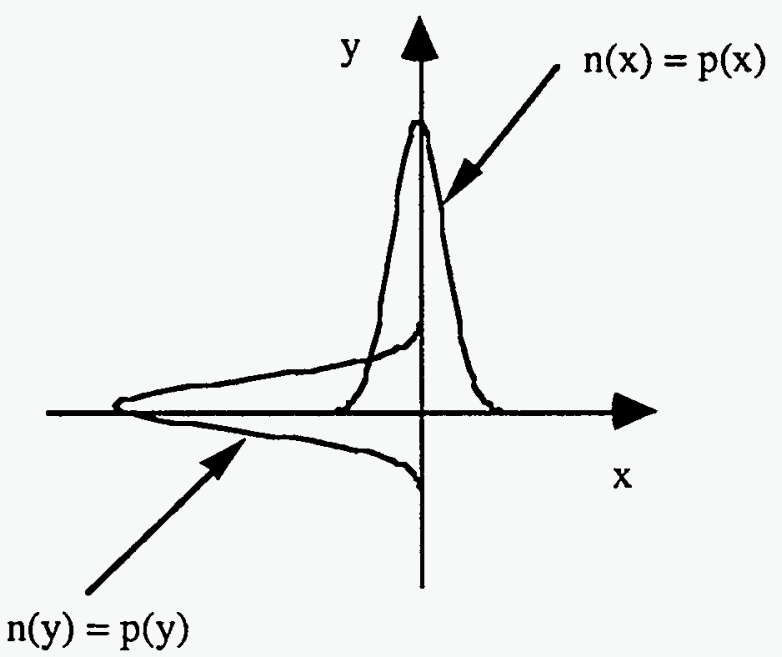

Figure 4.2.2: Schematic diagram of a two-dimensional model for the initial charge distribution about a heavy ion track. A) Plasma column from Fig. 4.2.1 collapsed as a disk B) Carrier density distributions in $\mathrm{x}$ - and $y$-directions in the disk with the origin at its center. 


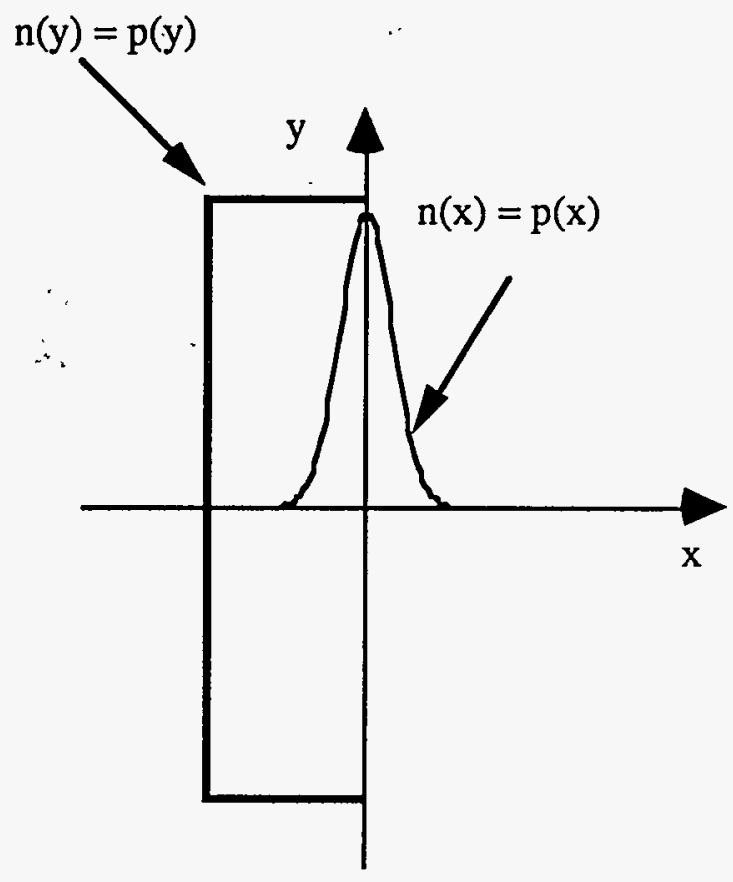

Figure 4.2.3: Schematic of a one-dimension approximation to the initial charge distribution about a heavy ion track. 


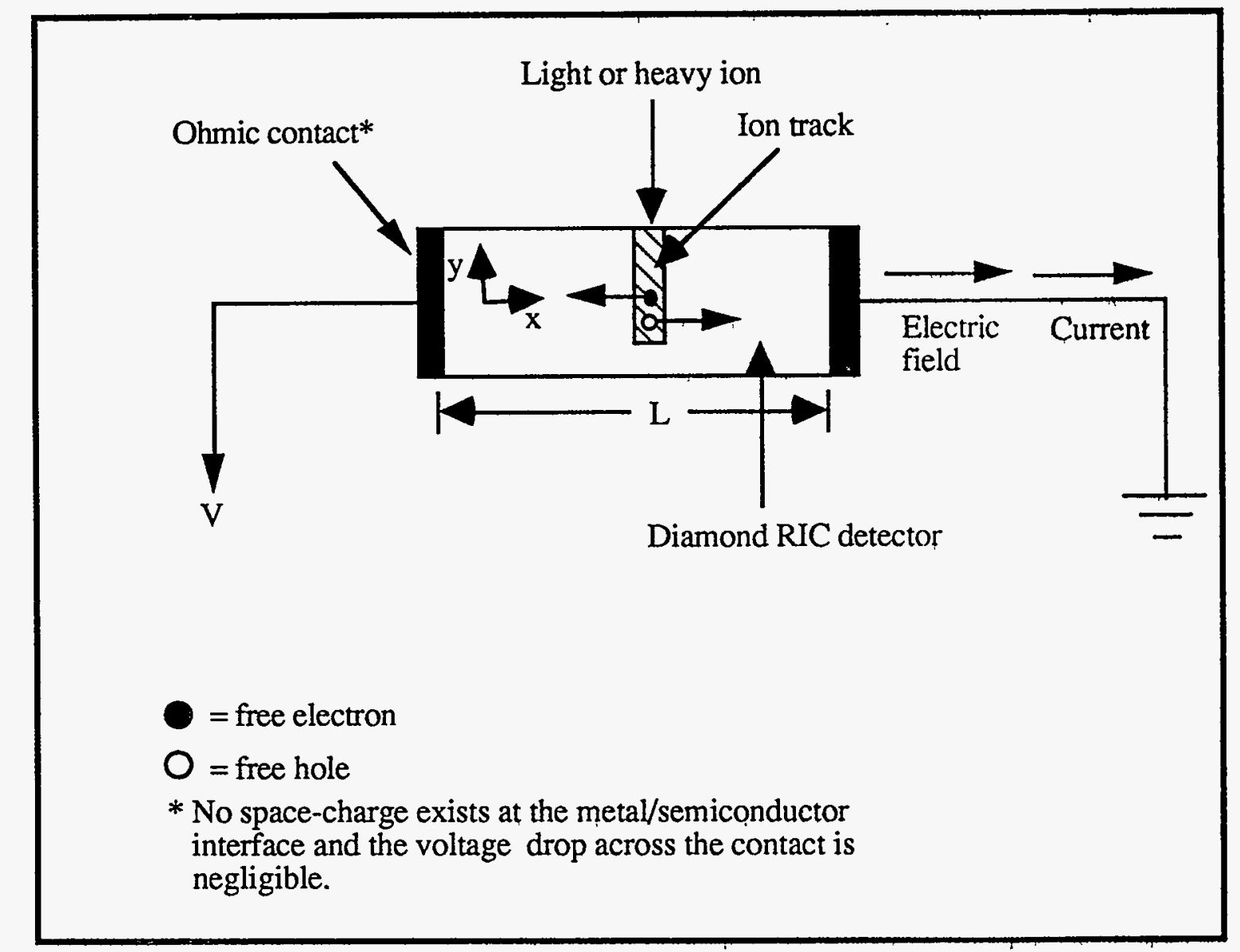

Figure 4.2.4: Schematic diagram of the one dimensional model for charge collection in a RIC detector. The detector width is $\mathrm{L}$ and the applied bias voltage is $\mathrm{V}$.

3) The electric field throughout the detector is assumed to be uniform and constant prior to the start of the transient.

4) The trajectory of an incident ion is linear and is normal to the electric field vector.

5) The track of a heavy ion is assumed to be linear and normal to the $x$ coordinate of Fig. 4.2.4. The density of free carriers generated by the ion is taken to be Gaussian-distributed along the $\mathrm{x}$-coordinate and constant along the particle's trajectory. Thus, 


$$
n(x)=n_{o} e^{-\left[(2.35)^{2}\left(x-x_{0}\right)^{2} /(F W H M)^{2} / 2\right]}
$$

where $n_{o}=$ peak density of the free carrier density distribution

$$
\begin{aligned}
& x=\text { spatial coordinate in the detector } \\
& x_{0}=\text { centroid of the free carrier density distribution. }
\end{aligned}
$$

FWHM = full-width-at-half-maximum of the free carrier density distribution.

The mean radius of the ion track, $r$, is assumed to be the FWHM. In doing so, greater than 0.97 of the total number of carriers produced is contained in the track. With either known or assumed mean ion range $(R)$ and mean radius of the track, $\mathrm{r}$, the mean volume of the ion track $\left(V_{r}\right)$ is given by

$$
V_{T}=\pi r^{2} R
$$

The initial number of carriers produced $\left(N_{l}\right)$ is just the ratio of initial ion energy to the mean energy required to produce an electron-hole pair assuming the ion is stopping within the detector. Therefore, the mean initial carrier density is given by

$$
\bar{n}=V_{T} / N_{l}
$$

The peak carrier density, $n_{o}$, is found by calculating the ratio between the peak and mean densities $\left(n_{0} / n\right)$ for a Gaussian distributed carrier density. This ratio is 2.76 (see appendix E). Conservation of the total number of carriers produced is inherent in this formulation because the peak density is normalized to the mean density which already includes the total number of carriers.

6) The centroid of the carrier distribution is located at the midpoint of the detector, i.e., at $\mathrm{x}=\mathrm{L} / 2$ 
7) For simplicity, all trapping/recombination is assumed to take place with the nitrogen impurity in the diamond. Nitrogen is known to be a deep donor in single-crystal, natural type IIa diamond, with an energy level at $2 \mathrm{eV}$ below the bottom of the conduction band [17]. Each trapping site is considered to have two possible charge states of +1 and 0 . Furthermore, due to the depth of the donor level, the holes trapped in these levels are fixed and no detrapping is considered to occur.

8) Three-body interactions, such as impact ionization and Auger recombination are neglected.

9) The detector operating temperature is taken as $300 \mathrm{~K}$.

10) The field-dependent mobility is taken to be that described in section 3.2 .

11) The effect of carrier-carrier scattering on mobility [1]] at high excitation densities is assumed to be described by

$$
\mu_{c c s}=3.11 \times 10^{16} \frac{T_{c}^{3 / 2}}{\sqrt{n p}}\left(\frac{1}{\ln \left[1+1.77 \times 10^{8}\left\{T_{c}^{2} /(n p)^{1 / 3}\right\}\right]}\right)
$$

where $\mathrm{n}, \mathrm{p}=$ free electron and hole densities, $\mathrm{cm}^{-3}$

$$
\mathrm{T}_{\mathrm{C}}=\text { carrier temperature, } \mathrm{K}
$$

The carrier temperature is calculated with Equ. 3.2.24

12) The surface recombination velocity at the detector boundaries is considered to be infinite so that the carriers reaching the contacts are immediately removed through recombination. 


\section{2,2 Model equations}

In any of the semiconductor texts previously mentioned, continuity equations for free carriers and Poisson's equation are found. We have chosen the forms of the equations given by Markowich [18]. The general continuity equations for free carriers are

$$
\begin{aligned}
& -\operatorname{div} J_{n}+e \frac{\partial n}{\partial t}=e G-e R_{n} \\
& \operatorname{div} J_{p}+e \frac{\partial p}{\partial t}=e G-e R_{p},
\end{aligned}
$$

and the general current equation for each carrier is defined as

$$
\begin{aligned}
& J_{n}=e \mu_{n}(\vec{n} \bullet \vec{E})-e D_{n}(\vec{\nabla} \bullet \vec{n}) \\
& J_{p}=e \mu_{p}(\vec{p} \bullet \vec{E})-e D_{p}(\vec{\nabla} \bullet \vec{p}),
\end{aligned}
$$

where $J_{n}=$ electron current density, $A / \mathrm{cm}^{2}$

$\mathrm{J}_{\mathrm{p}}=$ hole current density, $\mathrm{A} / \mathrm{cm}^{2}$

$\mathrm{e}=$ elementary charge, Coulombs

$\mathrm{n}=$ electron density, $\mathrm{cm}^{-3}$

$\mathrm{p}=$ hole density, $\mathrm{cm}^{-3}$

$\mathrm{R}_{\mathrm{n}}=$ electron density reduction rate, $\mathrm{cm}^{-3}-\mathrm{sec}^{-1}$

$\mathrm{R}_{\mathrm{p}}=$ hole density reduction rate, $\mathrm{cm}^{-3}-\mathrm{sec}^{-1}$

$\mathrm{G}=$ free carrier generation rate, $\mathrm{cm}^{-3}-\mathrm{sec}^{-1}$

$\mathrm{E}=$ electric field, $\mathrm{V} / \mathrm{cm}$

$D_{\mathrm{n}}=$ electron diffusivity, $\mathrm{cm}^{2} / \mathrm{sec}$

$\mathrm{D}_{\mathrm{p}}=$ hole diffusivity, $\mathrm{cm}^{2} / \mathrm{sec}$

$\mu_{\mathrm{p}}=$ hole mobility, $\mathrm{cm}^{2} / \mathrm{V}$-sec

$\mu_{\mathrm{n}}=$ electron mobility, $\mathrm{cm}^{2} / \mathrm{V}$-sec

Diffusivities of electrons and holes are calculated by use of the Einstein relation: 


$$
D_{i}=\frac{k T}{e} \mu_{i} \frac{F_{1 / 2}\left(\eta_{j}\right)}{F_{-1 / 2}\left(\eta_{j}\right)}
$$

where $i=n$ or $p$

$$
\begin{aligned}
& \mathrm{j}=\mathrm{c} \text { for conduction band } \\
& =\mathrm{v} \text { for valence band } \\
& \mathrm{E}_{\mathrm{F}}=\text { Fermi energy, } \mathrm{eV} \\
& \eta_{\mathrm{j}}=\frac{E_{j}-E_{F}}{k T} \\
& \mathrm{~F}_{1 / 2}\left(\eta_{\mathrm{j}}\right)=\text { Fermi-Dirac integral of order } \frac{1}{2}
\end{aligned}
$$

For energies larger than 4kT, the ratio of the Fermi-Dirac integrals in Equ. 4.2.11 is approximately unity. Since our simulated device has a energy gap of $5.5 \mathrm{eV}$ and the Fermi level is approximately at the middle of the band gap, the differences in energies between the band edges and the Fermi level are much greater than $4 \mathrm{kT}$. Therefore, Equ. 4.2.11 reduces to

$$
D_{i}=\frac{k T}{e} \mu_{i}
$$

The general Poisson's equation is written as

$$
\nabla^{2} \Psi=-\frac{e}{\varepsilon}(|\vec{p}|-|\vec{n}|-|\vec{c}|)
$$

where $\Psi=$ electric potential, $\mathrm{V}$

$$
\begin{aligned}
& \varepsilon=\text { permittivity }, F / \mathrm{cm} \\
& c=\text { doping profile }, \mathrm{cm}^{-3}
\end{aligned}
$$

Since the potential is defined by the Poisson's equation, the electric field is also defined by the second order Poisson's equation

$$
\vec{\nabla} \bullet \vec{E}=-\nabla^{2} \Psi=\frac{e}{\varepsilon}(|\vec{p}|-|\vec{n}|-|\vec{c}|)
$$

With these general equations, we can adequately describe the dynamic behavior of free carriers and the electric field in photoconductors. In the following, the 
vector notation will be deleted because we deal solely with one-dimensional transport equations.

At any time, the densities of free carriers are defined to be

$$
\begin{aligned}
& \mathrm{n}=\mathrm{n}_{\mathrm{o}}+\Delta \mathrm{n} \\
& \mathrm{p}=\mathrm{p}_{\mathrm{o}}+\Delta \mathrm{p}
\end{aligned}
$$

where $\mathrm{n}=$ total conduction electron density

$\mathrm{p}=$ total valence hole density

$\mathrm{n}_{\mathrm{o}}=$ thermal equilibrium conduction electron density

$\mathrm{p}_{\mathrm{o}}=$ thermal equilibrium valence hole density

$\Delta \mathrm{n}=$ excess conduction electron density from a particle interaction

$\Delta p=$ excess valence hole density from a particle interaction

Due to the large bandgap of diamond, and the complete absence of shallow levels

$\left(E_{\text {defect }}<k T\right.$ ), the thermal equilibrium concentration of free carriers is near zero. (In fact, if natural diamond was ideal intrinsic material, there would be about 1 carrier per volume of the earth.) Assuming that the resistivity of the detector material is $10^{10} \Omega-\mathrm{cm}$ and that carriers move at their saturation velocity of approximately $2 \times 10^{7} \mathrm{~cm} / \mathrm{sec}$, the thermal equilibrium carrier concentration would be about $31 \mathrm{~cm}^{3}$. Therefore, it is clear that we can neglect $n_{o}$ and $p_{0}$ during a transient and express the densities of free carriers solely in terms of excess carriers. With the assumption of a one-dimensional detector, and by applying assumptions 1 and 7 , equations $4.2 .7-4.2 .10$ are simplified to reald

$$
\begin{aligned}
& -\frac{\partial J_{n}}{\partial x}+e \frac{\partial \Delta n}{\partial t}=-e R_{n} \\
& \frac{\partial J_{p}}{\partial x}+e \frac{\partial \Delta p}{\partial t}=-e R_{p} \\
& J_{n}=e \mu_{n} \Delta n E+e D_{n} \frac{\partial \Delta n}{\partial x} \\
& J_{p}=e \mu_{p} \Delta p E-e D_{p} \frac{\partial \Delta p}{\partial x}
\end{aligned}
$$


The recombination rates given here are identical to those derived in Sec. 1.4.2.

In view of equations $4.2 .16-19$, the continuity equations can be written

$$
\begin{aligned}
& -\frac{\partial J_{n}}{\partial x}+e \frac{\partial \Delta n}{\partial t}=-e\left(B_{n} \Delta n n_{t r}^{+}\right) \\
& \frac{\partial J_{p}}{\partial x}+e \frac{\partial \Delta p}{\partial t}=-e\left(B_{p} \Delta p n_{t r}^{o}\right)
\end{aligned}
$$

where $n_{t r}^{\circ}=$ neutral trap density,

$$
\mathrm{n}_{\mathrm{tr}}^{+}=\text {positively-charged trap density }
$$

Because the charge states on the traps can change, the continuity equation for the traps is given by

$$
N_{t r}=n_{t r}^{+}+n_{t r}^{0}
$$

The density of positively-charged traps may be expressed in terms of the occupancy of the traps at thermal equilibrium and the change in occupancy due to the carriers generated by the incident radiation. This is expressed as

$$
n_{t r}^{+}=n_{t r o}^{+}+\Delta n_{t r}^{+}
$$

where $\Delta n_{t r}^{+}=$change of positively-charged trap density

$$
n_{t r_{0}}^{+}=\text {thermal equilibrium density of positively-charged traps }
$$

Upon substituting Equ. 4.2.22 and 4.2.23 into the continuity equations, one obtains

$$
\begin{aligned}
& -\frac{\partial J_{n}}{\partial x}+e \frac{\partial \Delta n}{\partial t}=-e\left(B_{n} \Delta n\left(N_{t r}-n_{t r_{0}}^{+}-\Delta n_{t r}^{+}\right)\right) \\
& \frac{\partial J_{p}}{\partial x}+e \frac{\partial \Delta p}{\partial t}=-e\left(B_{p} \Delta p\left(n_{t r_{0}}^{+}+\Delta n_{t r}^{+}\right)\right)
\end{aligned}
$$

Since the density of positively-charged traps is a dynamic variable, a rate equation is needed to describe it. The time rate of change of this density is simply the difference between the electron capture rate and the hole capture rate:

$$
\frac{\partial \Delta n_{t r}^{+}}{\partial t}=\left(B_{n} \Delta n\left(N_{t r}-n_{t r_{0}}^{+}-\Delta n_{t r}^{+}\right)\right)-\left(B_{p} \Delta p\left(n_{t r_{o}}^{+}+\Delta n_{t r}^{+}\right)\right)
$$


If we apply assumption 7, Equ. 4.2.25-27 reduce to

$$
\begin{aligned}
& -\frac{\partial J_{n}}{\partial x}+e \frac{\partial \Delta n}{\partial t}=-e\left(B_{n} \Delta n\left(N_{t r}-\Delta n_{t r}^{+}\right)\right) \\
& \frac{\partial J_{p}}{\partial x}+e \frac{\partial \Delta p}{\partial t}=-e\left(B_{p} \Delta p \Delta n_{t r}^{+}\right) \\
& \frac{\partial \Delta n_{t r}^{+}}{\partial t}=\left(B_{n} \Delta n\left(N_{t r}-\Delta n_{t r}^{+}\right)\right)-\left(B_{p} \Delta p \Delta n_{t r}^{+}\right)
\end{aligned}
$$

Finally, we can write the Poisson's equation for a one-dimensional detector with singleenergy traps--which, in essence, describes the doping profile-- to complete the set of modeling equations:

$$
\frac{\partial E}{\partial x}=-\frac{\partial^{2} \Psi}{\partial x^{2}}=\frac{e}{\varepsilon}\left(\Delta p-\Delta n+\Delta n_{t r}^{+}\right)
$$

Because of the spatial invariant property of the total current, we can write

$$
\begin{aligned}
J_{T}(t)=J_{p}(x, t) & +J_{n}(x, t)+J_{D}(x, t), \\
\text { where } J_{D}(x, t) & =\varepsilon \frac{\partial E}{\partial t} \\
& =\text { displacement current }
\end{aligned}
$$

The displacement current is due to the time dependence of the electric field distribution during the relaxation processes.

Given the set of governing equations above, boundary and initial conditions must be specified to complete the mathematical formulation of the model. The boundaries of the modeled system are the electrical contacts. These are taken as non-injecting ohmic contacts. They are ohmic only in the sense that the contact resistance is considered to be negligible compared to that of the detector. No boundary conditions need be specified for the equation describing the density of trapped carriers because this equation is a consequence of the carrier continuity equations. Translating these conditions into a mathematical form and using the initial conditions specified in the model assumptions, we arrive at the following for both the initial and boundary conditions: 


$$
\begin{aligned}
& \Delta \mathrm{n}(0, \mathrm{t})=\Delta \mathrm{n}(\mathrm{L}, \mathrm{t})=\Delta \mathrm{p}(0, \mathrm{t})=\Delta \mathrm{p}(\mathrm{L}, \mathrm{t})=0 \\
& \Delta n(x, 0)=\Delta p(x, 0)=n(x) \\
& \Delta n_{t r}^{+}(x, 0)=0 \\
& \Psi(0, t)=0, \Psi(L, t)=V
\end{aligned}
$$

where $n(x)$ is given in assumption 5 .

The set of continuity equations along with Poisson's equation form a system of non-linearly coupled, elliptic/parabolic partial differential equations for which analytical solutions are not available. Solutions must be obtained by numerical approximation. As indicated above, we have used the formulation given in Ref. 11 to analyze the response of diamond RIC detectors under local illumination caused by detector-ion interactions. Before solving this set of coupled partial differential equations, the equations are nondimensionalized. The time is non-dimensionalized by dividing the physical time by a normalization time, $\tau$, which is chosen to be $1 \mathrm{ps}$. Following the initial formulation given in Ref. 11 where a model similar to the one presented in this section is applied to examine the response of photoconductor excited uniformly with the excitation pulse having a finite width in time, the free carrier generation rate in Equ. 4.2.7 or 4.2.8 is taken as the constant go. But in a case of an impulse excitation, the free carrier generation rate becomes merely a scaling factor for non-dimensionalization. The electric field, E, is nondimensionalized with the initial applied field, $V / L$. The positively-charged trap density, $\Delta n_{t r}^{+}$is non-dimensionalized with the total trap density, $N_{T}$. The numerical method used in Ref. 11 to solve the partial differential equations is described in some detail in the next section.

4.2.3 Numerical solution to the model equations

Iverson used the exponentially weighted, spatial-finite-upwind-differencing scheme of Scharfetter and Gummel[42] to discretize the system of equations for the current densities (see Equ. 4.2.27 and 4.2.28). This method of discretization has been 
shown to be numerically stable unconditionally, whereas the common finite difference scheme can be shown to be numerically unstable if the potential change between any two mesh points in the spatial grid is greater than $2 \mathrm{kT} / \mathrm{e}$. The stability comes from exponentially weighting the mesh points whereby a large change in the potential between any two adjacent mesh points is damped so that the current density midway between the two adjacent points approaches the drift current at either mesh points i or $\mathrm{i}-1$. This process results in a non-linearly coupled set of first-order ordinary differential equations in time, and algebraic equations resulting from Poisson's equation. These two sets of equations were then solved simultaneously and were integrated forward in time using Gear's integration algorithm.

The discretization of the equations was done on a spatially non-uniform grid. The detector width is divided into 100 segments (or 101 mesh points). Each mesh point is related to the spatial coordinate $x$, of Fig. 4.3 .1 by

$$
\frac{x}{L}(i, \xi)=\frac{1}{2}\left(\left(\frac{\left(\operatorname{ATANH}\left(2\left(i-\frac{1}{2}\right) \xi\right)\right)}{\operatorname{ATANH}(i)}\right)+1\right)
$$

where $\mathrm{i}=$ mesh point

$\xi=$ non-uniformity parameter

With this approach, higher resolution may be achieved in areas of large perturbation simply by assigning a higher density of mesh points to such regions by varying the nonuniformity parameter $\xi$. Regions with the largest initial perturbations could be estimated from the formulation of the conductivity modulation problem, and it is expected that they should not vary to a great extent during the progress of a computation. In our case, with Gaussian-distributed carrier densities centered at the midpoint of the detector, a denser 
grid near that region was used. As seen in Fig 4.2.5, this particular generating function produces a much denser grid at the midsection than at its boundaries.

The temporal grid contains points that are distributed uniformly with the grid width $\Delta t$ between 0 and the specified simulation termination time, $T$. However, the Gear's integration routine will achieve a self-consistent solution to the model thereby advancing the solution until the simulation time arrives at $t+\Delta t$. At this point, the carrier and positively-charged trap densities and the potential profiles are written to a computer file and another call is made to the Gear routine to solve for model up to the next time step. After the simulation time reaches $\mathrm{T}$, the densities and the potential profiles are further processed to calculate relevant profiles such as current density and electric field. For majority of the calculations, $\Delta \mathrm{t}$ was taken to be $5 \mathrm{ps}$.

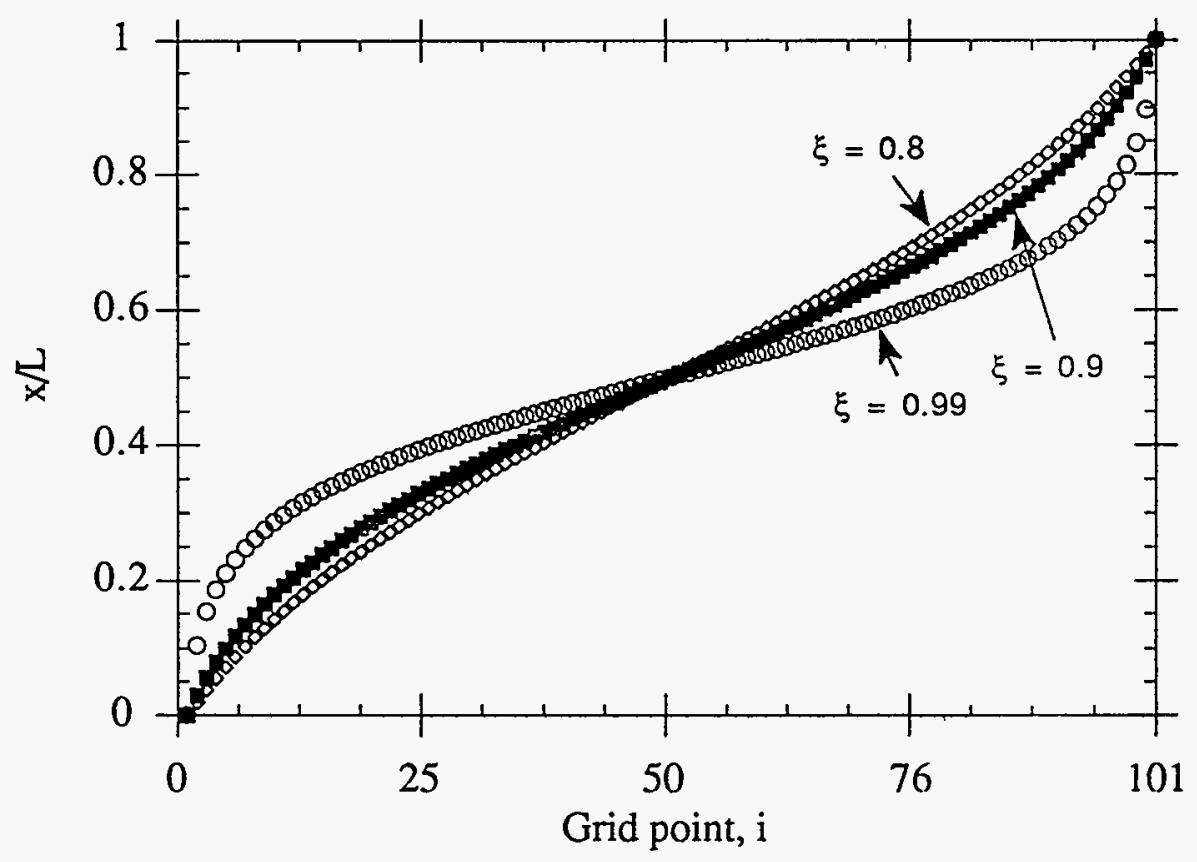

Figure 4.2.5: Distribution of the grid points (totaling 101) along the spatial axis. As $\xi$ approaches unity, an ever greater number of grid points is allocated to the center of the spatial axis. 
4.3 Model calculations and comparisons with experimental results

When either a $5-\mathrm{MeV} \mathrm{He}^{+}$- or $10-\mathrm{MeV} \mathrm{Si}^{3+}$ - ion is stopped within the detector, a dense electron-hole plasma is formed along the ion's track before any relaxation takes place. Under the influence of the applied field, the free electrons and holes migrate until the resultant charge separation induces a self-field equal in magnitude but opposite in direction to the applied field. At this point, the net electric field at the core of the ion track should approach zero due to the shielding of the applied field by the charge carriers located near the outer parts of this so-called space-charge region. The collapse of the electric field can be referred to as dielectric collapse and should occur on the time scale of the dielectric relaxation time characteristic of the free carrier density. This is the time scale in which a new equilibrium value of the electric field is reached. Since the collapsing electric field during the charge separation represents a time-varying electric field, a displacement current will be generated. Therefore, we can expect initially, that the total current will have components due to drift, diffusion, and the displacement current. Following collapse of the electric field, the majority of the current should be due to drift and diffusion. At all times, trapping and recombination of the electrons and holes will takẹ place.

This physical picture has been examined quantitatively for the two different types of ion excitations studied experimentally by solving the model equations described in Sec. 4.2. In this section, the results of calculations with the model are presented and compared with the results from the experiments discussed in Sec. 4.1.

\subsubsection{5-MeV He${ }^{+}$-ion excitation}

Experimental data on current transients with $\mathrm{He}^{+}$ions interacting with the diamond detector were compared to the transients calculated with the model described above using the material parameters given in Table 4.3.1. In the absence of detailed 
knowledge on the actual initial charge distribution about an average track, the charge distribution was taken as a radially symmetric Gaussian with variable width (= the mean radius of the track) and corresponding peak density, $n_{q}$, defined by use of the mean track length and the requirement that the initially produced total charge remain constant. The widths of the Gaussian were varied in the range $1-3 \mu \mathrm{m}$ to find the best fit of the calculated transient to the experimental data. In Table 4.3.2, the various track widths and corresponding peak carrier densities, $n_{o}$, that have been tested.

Table 4.3.1: Computational parameters used in model calculations for $5 \mathrm{MeV} \mathrm{He}^{+}$-ion excitation. In order, the columns show the low-field electron mobility $\left(\mu_{\mathrm{oe}}\right)$, the low-field electron mobility $\left(\mu_{\mathrm{oh}}\right)$, the electron capture cross section $\left(\sigma_{\mathrm{e}}\right)$, the electron capture cross section $\left(\sigma_{\mathrm{h}}\right)$, the total nitrogen concentration $\left(\mathrm{N}_{\mathrm{T}}\right)$, the carrier generation rate $\left(\mathrm{g}_{\mathrm{o}}\right)$ (as mentioned in Sec. 4.2.3), and the detector width ( $\mathrm{L}$ ).

\begin{tabular}{|ccccccc|}
\hline $\begin{array}{c}\mu_{\mathrm{oe}} \\
\left(\mathrm{cm}^{2} / \mathrm{V}-\mathrm{sec}\right)\end{array}$ & $\begin{array}{c}\mu_{\mathrm{oh}} \\
\left(\mathrm{cm}^{2} / \mathrm{V}-\mathrm{sec}\right)\end{array}$ & $\begin{array}{c}\sigma_{\mathrm{e}} \\
\left(\mathrm{cm}^{2}\right)\end{array}$ & $\begin{array}{c}\sigma_{\mathrm{h}} \\
\left(\mathrm{cm}^{2}\right)\end{array}$ & $\begin{array}{c}\mathrm{N}_{\mathrm{T}} \\
\left(\mathrm{cm}^{-3}\right)\end{array}$ & $\begin{array}{c}\mathrm{g}_{\mathrm{o}} \\
\left(\mathrm{cm}^{-3} / \mathrm{sec}\right)\end{array}$ & $\begin{array}{c}\mathrm{L} \\
(\mu \mathrm{m})\end{array}$ \\
\hline 500 & 370 & $2 \times 10^{-13}$ & $5 \times 10^{-16}$ & $3 \times 10^{18}$ & $1.15 \times 10^{28}$ & 20 \\
\hline
\end{tabular}

Table 4.3.2: Radius of ion track, $r$, and corresponding initial peak carrier density, $n_{o}$.

\begin{tabular}{|cc|}
\hline $\mathrm{r}(\mu \mathrm{m})$ & $n_{o}\left(10^{16} \mathrm{~cm}^{-3}\right)$ \\
\hline 1 & 2.60 \\
1.5 & 1.15 \\
1.8 & 0.80 \\
2 & 0.65 \\
3 & 0.29 \\
\hline
\end{tabular}


In order to compare with the experimental results, the calculated current transients were first smoothed with a Gaussian function with the FWHM of 1.3 ps to approximate the smoothing expected from the finite stopping time $(\approx 1.3 \mathrm{ps})$ of the ion, and then this distribution was smoothed with a second Gaussian function representing the impulse response of the recording system with the rise time of $18.6 \mathrm{ps}$. Also, current densities were converted to currents by using the projected area $(A=2 r R)$ of the ion track that is normal to the $\mathrm{x}$-axis. Fig. 4.3.1 shows the comparisons between the experimental data and calculated transients for the various track widths. The best agreement between model and experiment appears to occur with the track width of $1.5 \mu \mathrm{m}$. The radius of the $\alpha$-particle tracks are generally believed to be in the range $0.1-2 \mu \mathrm{m}[19,20,21,22,23]$. Therefore, the estimated He-ion track radius appears to be consistent with the range of reported values. All subsequent calculations in this section will use this track radius.

It is evident from the calculations that the magnitude of the current and the total charge collected increase substantially with increasing width of the distribution. These effects are expected from the reduced space-charge fields that are generated with the wider, less dense plasmas. The main difference in all of the transients is that the second peaks in the transients appear earlier in time for larger FWHM. Again, this is consistent with the greater plasma erosion expected with larger FWHM due to the greater penetration of the applied field.

With the track width estimated, the results representing the major quantities of interest associated with the transient are shown in Fig. 4.3.2-4.3.4 for an applied field of $50 \mathrm{KV} / \mathrm{cm}$. These quantities are temporal and spatial variations of carrier densities, electric field and positively-charged trap density. Fig. 4.3.2 shows the temporal and spatial variations of the densities of electrons and holes calculated with the model. Both indicate marked reductions with time over the first $50 \mathrm{ps}$ following production, with reduction in the densities of holes occurring at a significantly greater rate than for electrons. It is also apparent that the carrier distributions exhibit tails that extend from the 


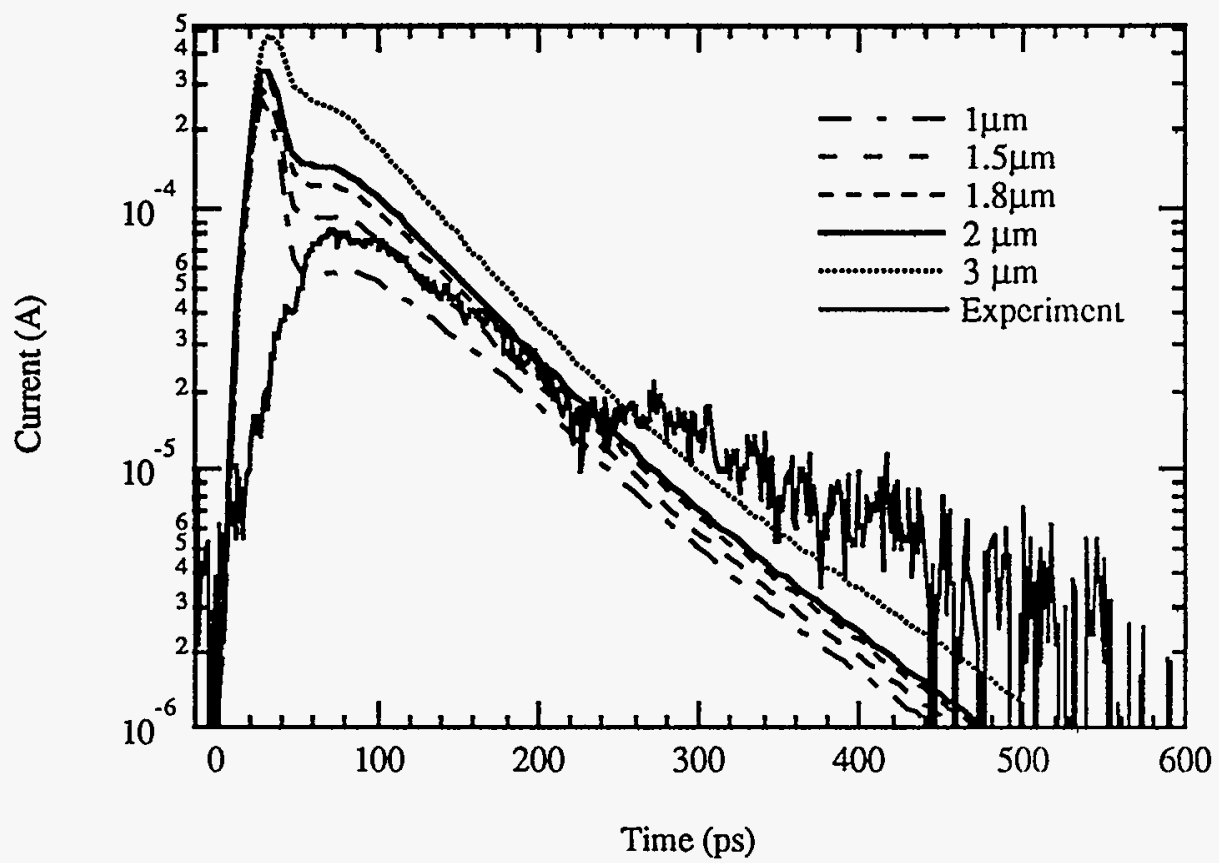

Figure 4.3.1: Calculated current transients as a function of the width (FWHM) of the Gaussian distribution of the initial free-carrier density ; the applied electric field was $50 \mathrm{KV} / \mathrm{cm}$. All transients were smoothed with a Gaussian of the width 1.3 ps representing the ion stopping followed by additional smoothing with a Gaussian of the width 18.6 ps representing the system response function.

region of production to the contact toward which they drift under the influence of the applied field. The densities in the tails are much smaller than those near the site of production over the first few hundred picoseconds following production. Overall, the calculations indicate that bulk trapping/recombination near the site of carrier production is a dominant feature of the dynamics of carrier transport and suggest that such an effect is relatively less significant in other regions of the detector.

The preferential loss of holes during the initial stages of the transient follow from the model assumption that nitrogen is the only active trapping site. As indicated in 
(A)

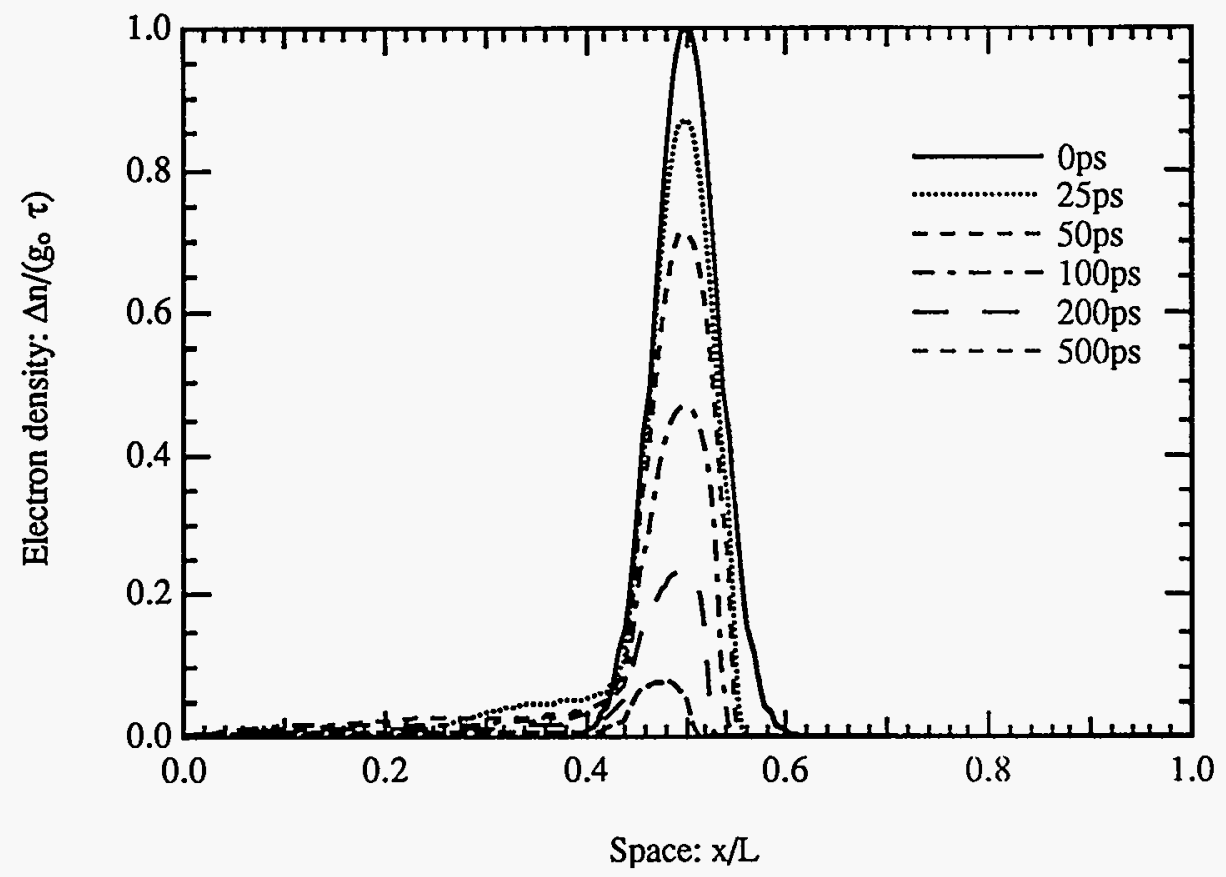

(B)

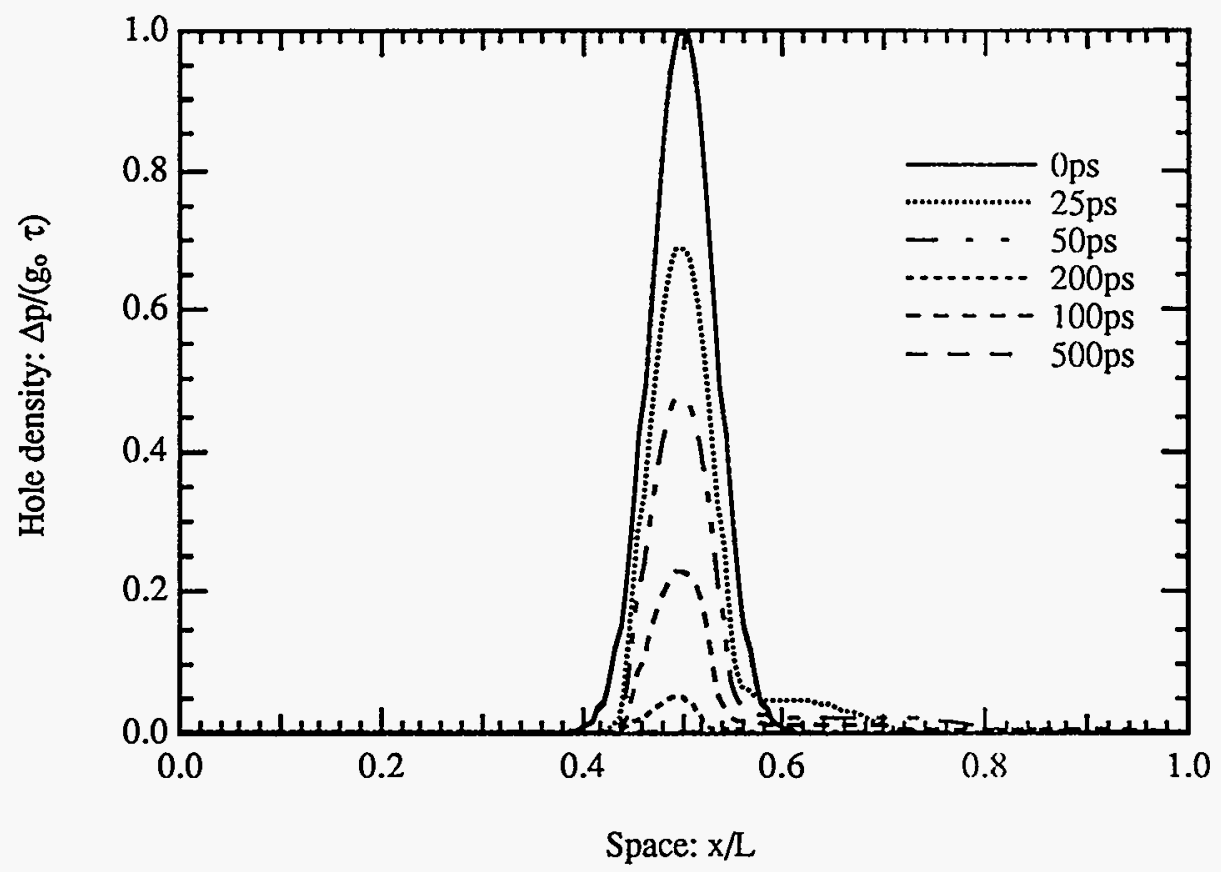

Figure 4.3.2: Calculated carrier density distribution as a function of space and time; (A) Electron density distribution; (B) hole density distribution. 
Assumption 7, nitrogen is assumed to be initially neutral, and to exist only in charge states of 0 and $1^{+}$. Consequently, only free holes can be captured initially. Later, as the density of positively-charged sites increases by continuing capture of holes, electron trapping can take place at a significant rate.

In Fig. 4.3.3 the calculated temporal and spatial variation of the electric field in the detector is shown. Within a few picoseconds of charge production, the electric field near the centroid of the initial carrier distribution collapses and is held at near zero for well over $500 \mathrm{ps.}$ This is clearly due to the space-charge field developed as a result of the initial charge separation. The large spatial dependence of the electric field is qualitatively consistent with the initial Gaussian charge distribution, the transport of charge away from its centroid, and the carrier recombination noted above. As time progresses, the electric field at $(x / L)>0.5$ is seen to increase markedly whereas the field for $(x / L) \geq 0.5$ decreases continuously. This can be related to the distribution of trapped holes as indicated in Fig. 4.3.4 where it is seen that some $10 \%$ of the initial number of holes becomes trapped and leads to a fixed charge distribution in the detector over the time scale considered. If the calculations were allowed to progress to very large times, the final electric field distribution would be dictated by the spatial density of the trapped holes and the applied potential, because all electrons will have recombined or migrated to a contact. This is a direct result of the non-injecting character of the electrical contacts, the absence of detrapping (assumption 7) and the absence of any source of leakage into the detector. It is important to remember that the non-injecting character comes from the assumption that the surface recombination velocity at the contacts is considered to be infinite thereby removing any carrier reaching the contacts through recombination at the detector boundary (assumption 12). In reality, the finite conductivity of diamond will allow leakage currents and the electric field will eventually recover to that defined by the applied potential. 
(A)

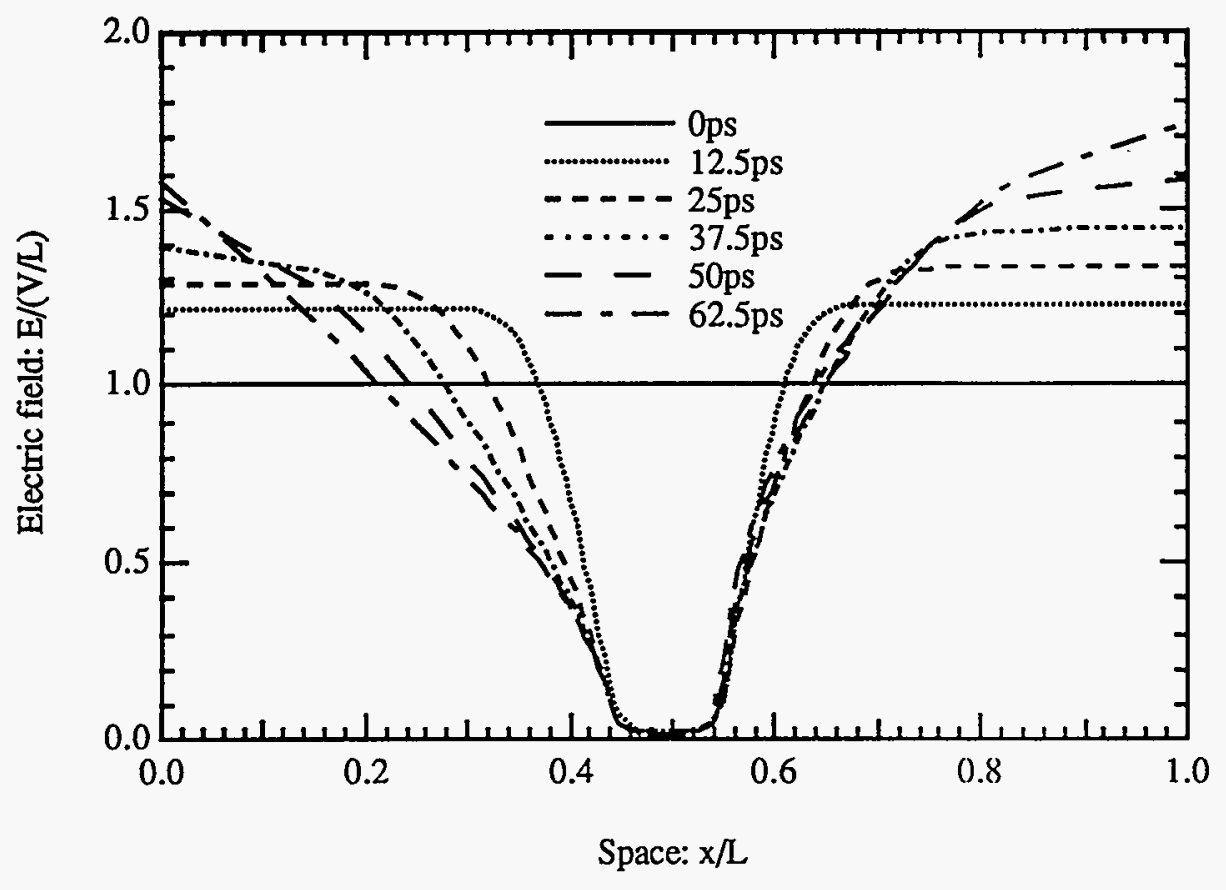

(B)

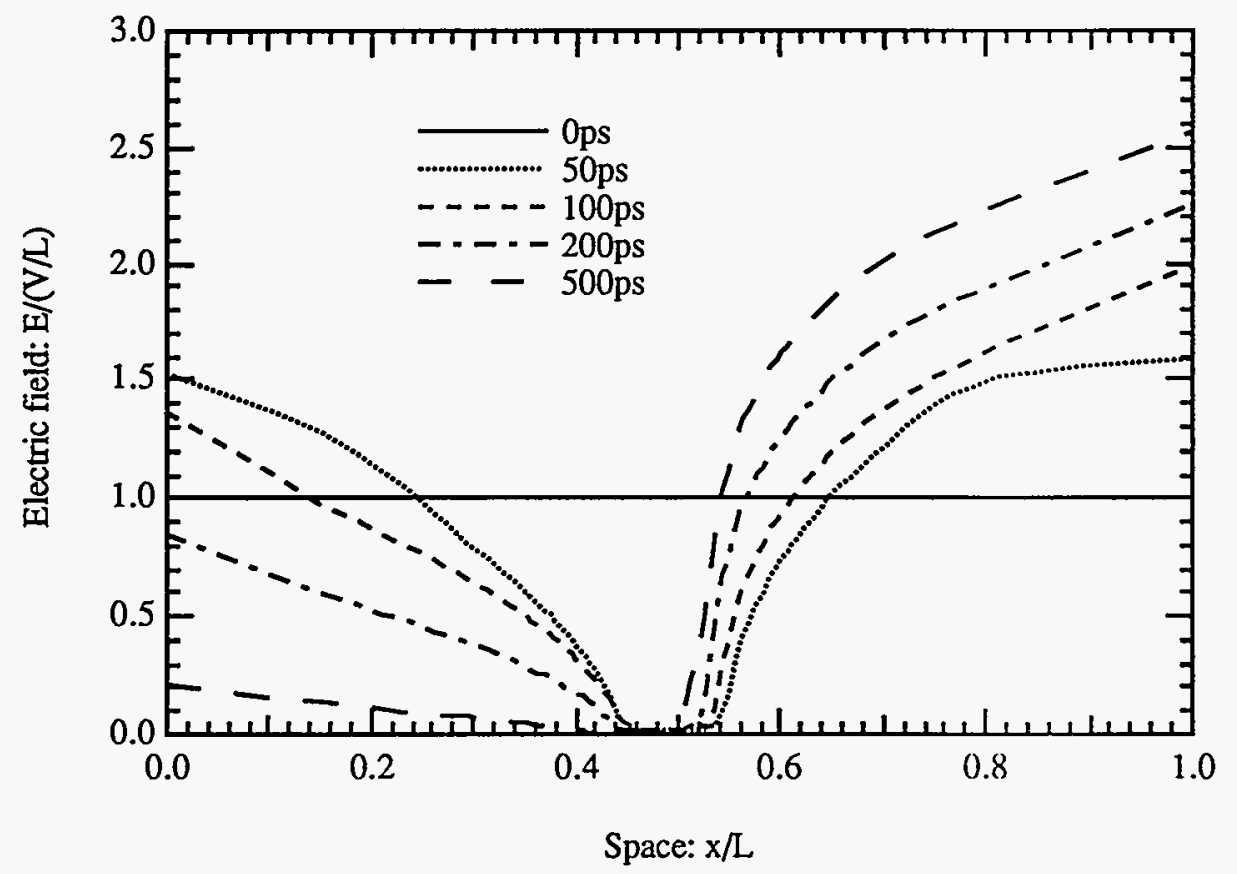

Figure 4.3.3: Calculated Electric field distribution as a function of space and time. A) Electric field distribution as a function of time and space in the range $0-62.5 \mathrm{ps}$. B) Electric field distribution as a function of time and space in the range $0-500$ ps. 


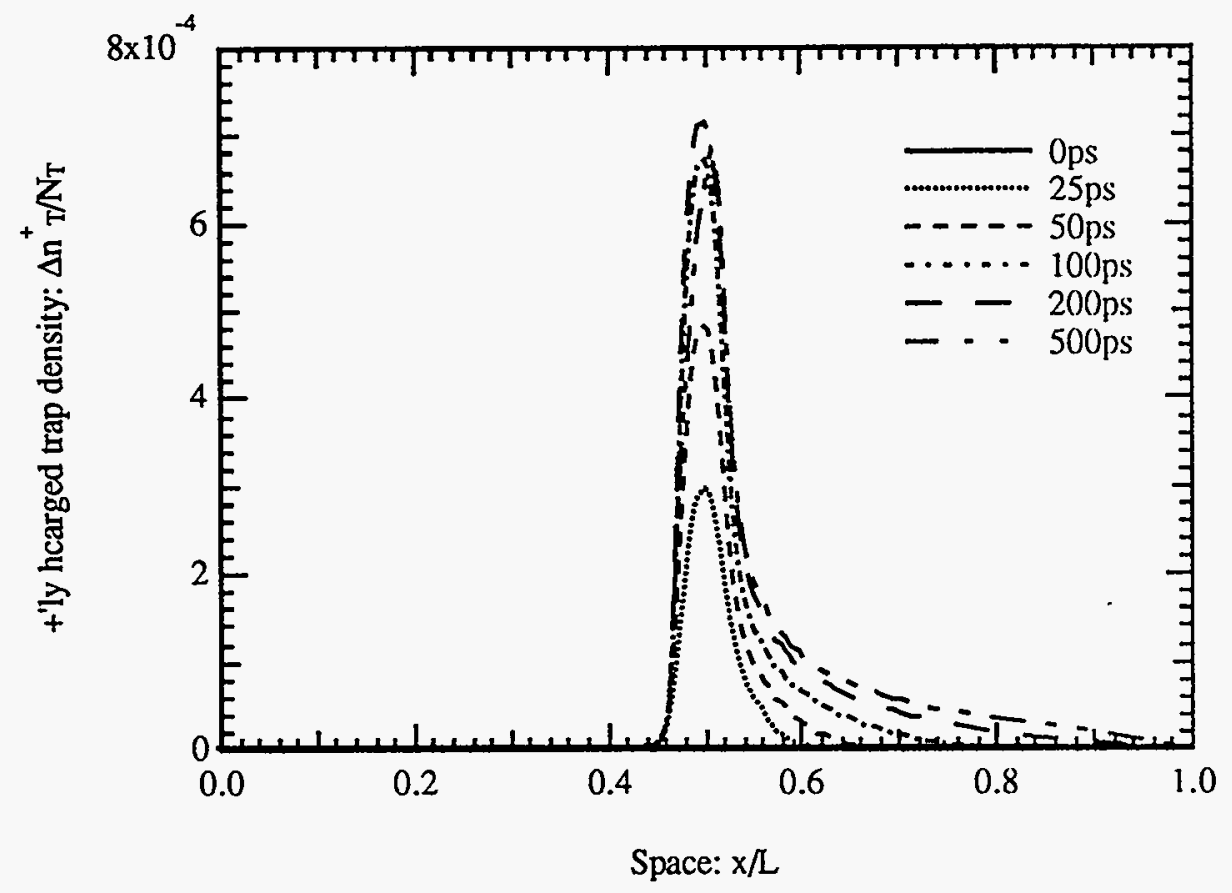

Figure 4.3.4: Calculated positively-charged trap density distribution as a function of space and time.

The temporal variation of the current density induced at the contacts is shown in Fig. 4.3.5. The large current density at $\mathrm{t}=0$ is due to transport of the initial carrier densities. Within times less than $5 \mathrm{ps}$, the current density decreases by a factor of 6 (inset of Fig. 4.3.5), a direct result of the electric field collapse from buildup of space charge. Although the initial currents are very large, the total charge collected during the first 10 ps represents only about $10 \%$ of that calculated for the entire transient.

Following the initial transient, a peak is observed centered at about $50 \mathrm{ps}$ later. This is due predominantly from drift and diffusion of carriers near the outer extent of the carrier distribution where the electric field has not completely collapsed. The fact that the transient displays a peaked form is due to the increase in electric field at the outer reaches of the carrier distribution after short times. Finally, the decay of the current density at longer times is primarily the result of trapping and recombination at deep donor sites. 


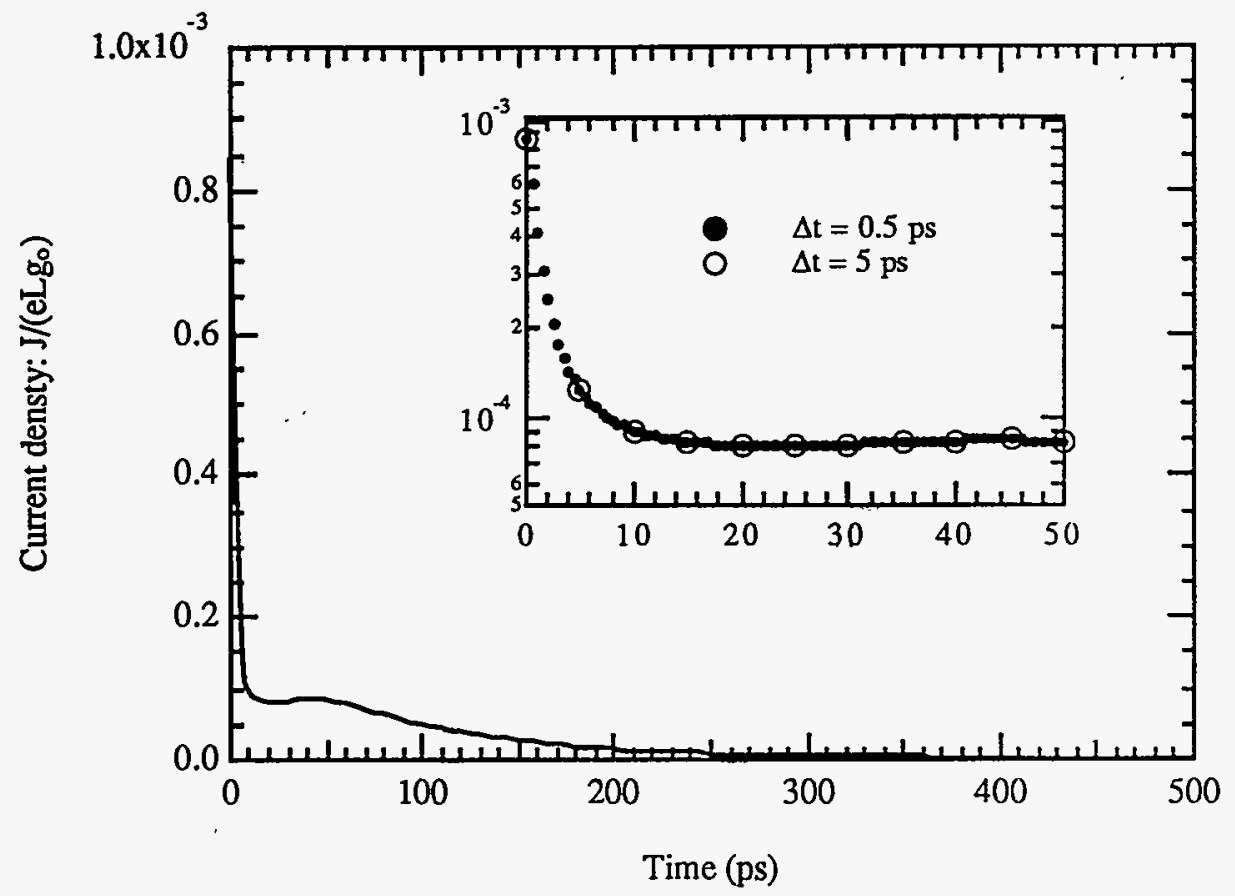

Figure 4.3.5: Calculated instantaneous current density. The inset shows calculated current density magnified in the time interval 10 50 ps calculated with two different temporal grid widths; one is $\Delta t=0.5 \mathrm{ps}(\mathrm{o})$ and the other being $\Delta \mathrm{t}=5 \mathrm{ps}(\bullet)$.

The magnitude and temporal variation of the current densities at short times are strongly dependent upon the assumptions built into the calculation and are expected to differ significantly from the initial response of a real detector. First, the model assumes that the free carrier densities are fully present at $t=0$. In reality, the time required for stopping of heavy ions is about 1-2 ps. In the limit of zero coupling between ions produced along the ion's trajectory, the detector response will be due to the summation of response functions similar to that shown in Fig. 4.3.5, but with their origins displaced to increasingly larger times. Taking into account the coupling that will affect the transport of carriers along the entire track, we can expect to see a markedly smaller and slower transient in the response of a real detector due to the occurrence of dielectric relaxation from the beginning of the ion trajectory. Therefore, the calculated response shown here 
must be viewed with caution and is certain to overestimate the current that can be drawn at early times.

It is also important to note that the magnitude of the initial intensity may be affected by the choice of the temporal grid used in the calculations. It is clear from the inset of Fig. 4.3.5 that with the choice of grid width of $\Delta t=5 \mathrm{ps}$, the initial current density decreases by about an order of magnitude within the first two recorded grid points. When the temporal grid width is decreased to $\Delta t=0.5 \mathrm{ps}$, the initial current density decreases only a factor of about 1.3 in $0.5 \mathrm{ps}$. Therefore, with $\Delta t=0.5 \mathrm{ps}$ the area under the current density profile for the first $15 \mathrm{ps}$ is considerably smaller than when $\Delta \mathrm{t}$ is taken as $5 \mathrm{ps}$. In point of fact, the area under the transient calculated with $\Delta t=0.5 \mathrm{ps}$ is smaller by about a factor of 2 in comparison to the one calculated with $\Delta t=5 \mathrm{ps}$ for the first $15 \mathrm{ps}$. If now the transient shown for the $\Delta t=0.5$ grid is smoothed with the response functions as previously mentioned, the intensity of the initial peak should be lower than with a grid with $\Delta t=5$ ps by about a factor of 2 . This reduction in the initial peak intensity is shown in Fig. 4.3.6-A and 4.3.6-B. An exponential fit to the first 15 ps was also performed in an attempt to approximate the calculated transient with a finer temporal grid. The quality of the fit to the first 15 ps of the transient with $\Delta t=5$ ps is shown in Fig. 4.3.7. In Fig. 4.3.7$\mathrm{C}$, the result of the exponential approximation is shown for the smoothed current transient. The approximation is within about 1.07 of the smoothed transient with $\Delta t=0.5$ ps with respect to the intensity of the first peak. Therefore, this approximation will be used for comparing calculation with measured current transients for fields in the range $\pm(75-100) \mathrm{KV} / \mathrm{cm}$ instead of performing additional calculations with the finer temporal grid.

As previously mentioned, any coupling between the electron-hole pairs produced along the ion trajectory is to result in a reduced amplitude for initial part of the current, transient. The effect of such reduction was examined artificially by reducing the intensity 
of the instantaneous current density at $t=0$. For example, if the initial instantaneous current density shown in Fig. 4.3.5 is reduced by a factor of 8 and then convolved with

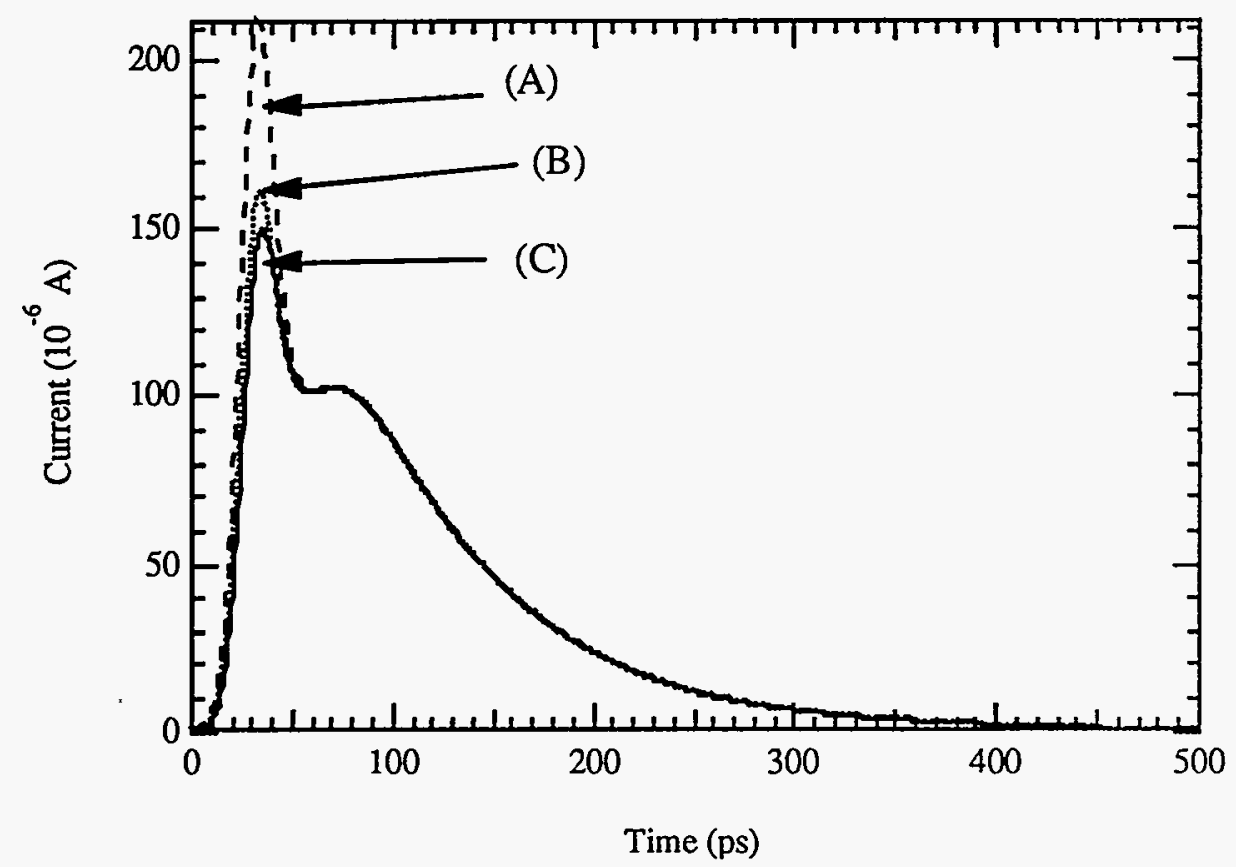

Figure 4.3.6: Temporal variation of the calculated current transient smoothed with a Gaussian function with rise time of 1.3 ps representing ion stopping time followed by smoothing with a Gaussian function representing the impulse response of the recording system with the rise time of $18.6 \mathrm{ps}$; (A) temporal grid width, $\Delta \mathrm{t}=5 \mathrm{ps}$; (B) same calculation as (A) but with an exponential fit to the first 3 points to approximate the current output over the first $10 \mathrm{ps}$; (C) temporal grid width, $\Delta \mathrm{t}=0.5 \mathrm{ps}$. Applied electric field $=50 \mathrm{KV} / \mathrm{cm}$. 


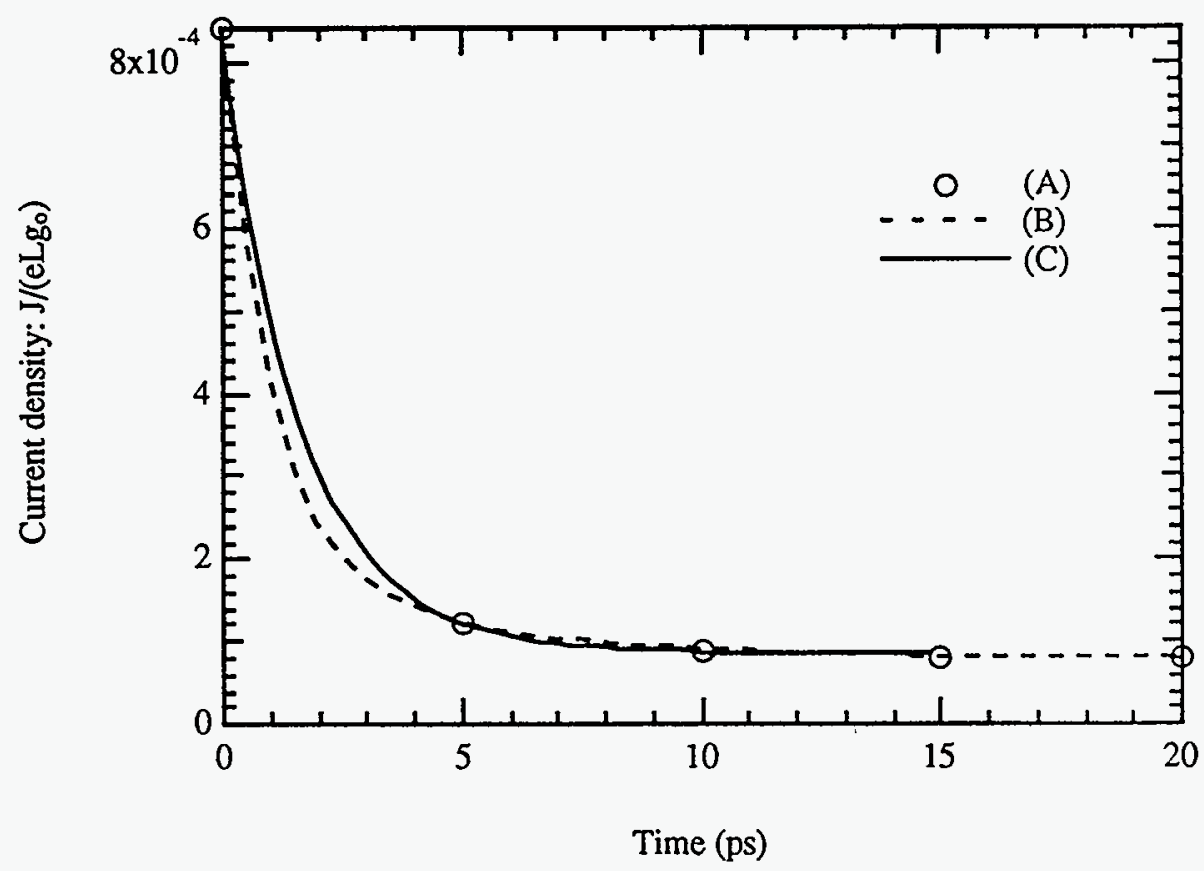

Figure 4.3.7: Calculated current density output for two different temporal grid widths; (A) $\Delta t=5 \mathrm{ps}$; (B) $\Delta t=0.5 \mathrm{ps;}$ (C) fitted the first $15 \mathrm{ps}$ of the profile shown in(A) with an exponential function to approximate the shape of the current density for the first $5 \mathrm{ps}$ of the profile shown in (B).

the Gaussian response functions representing the ion stopping and the recording system response, the distribution shown in Fig. 4.3.8 is obtained. It is clear that the initial system rise-time limited peak nearly disappears and the second peak begins to dominate the current profile. The corresponding rise time increases from $18.6 \mathrm{ps}$ to about $24 \mathrm{ps}$. If the amplitude of the initial instantaneous current is further reduced, the amplitude of the first peak in Fig. 4.3 .5 becomes correspondingly reduced. In the limit that the initial current seen in Fig. 4.3.5 approaches zero, the rise time of the calculated current would be about 50 ps (where the centroid of the second peak lies) which is about what is measured experimentally. 


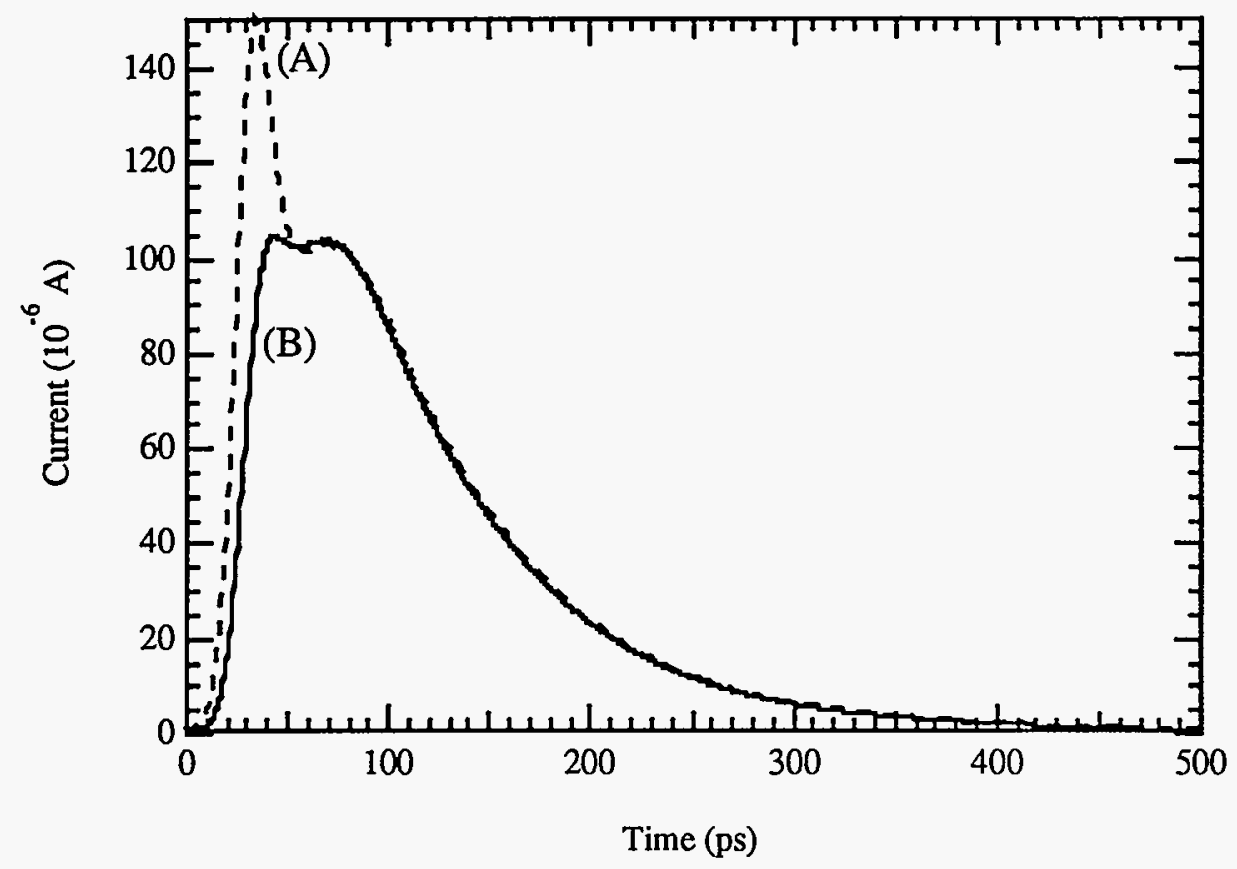

Figure 4.3.8: Temporal variation of the calculated current convolved with the Gaussian functions describing the ion stopping and the recording system response: (A) this profile is identical to Fig. 4.3.5; (B) the initial amplitude shown in Fig. 4.3.4 was reduced by a factor of 8 then convolved with Gaussian response functions.

The effects of plasma erosion on the response of the detector is shown in Fig. 4.3.9. The transients were calculated as a function of applied electric field in the range $(50-100) \mathrm{KV} / \mathrm{cm}$ with the width of the initial carrier density distribution fixed at 1.5 $\mu \mathrm{m}$. It is evident from the calculation that the second peak shifts somewhat to earlier times with increasing field. This is due to the fact that the plasma erosion rate is larger for the larger fields. The qualitative characteristics of the transients are similar to those seen in Fig. 4.3.1 for times less than $100 \mathrm{ps}$ which indicates the importance of plasma screening at the earlier times. At times greater than $100 \mathrm{ps}$, the transient decay times for the transients decrease with increasing fields. As seen in Fig. 4.3.2, a fraction of the 


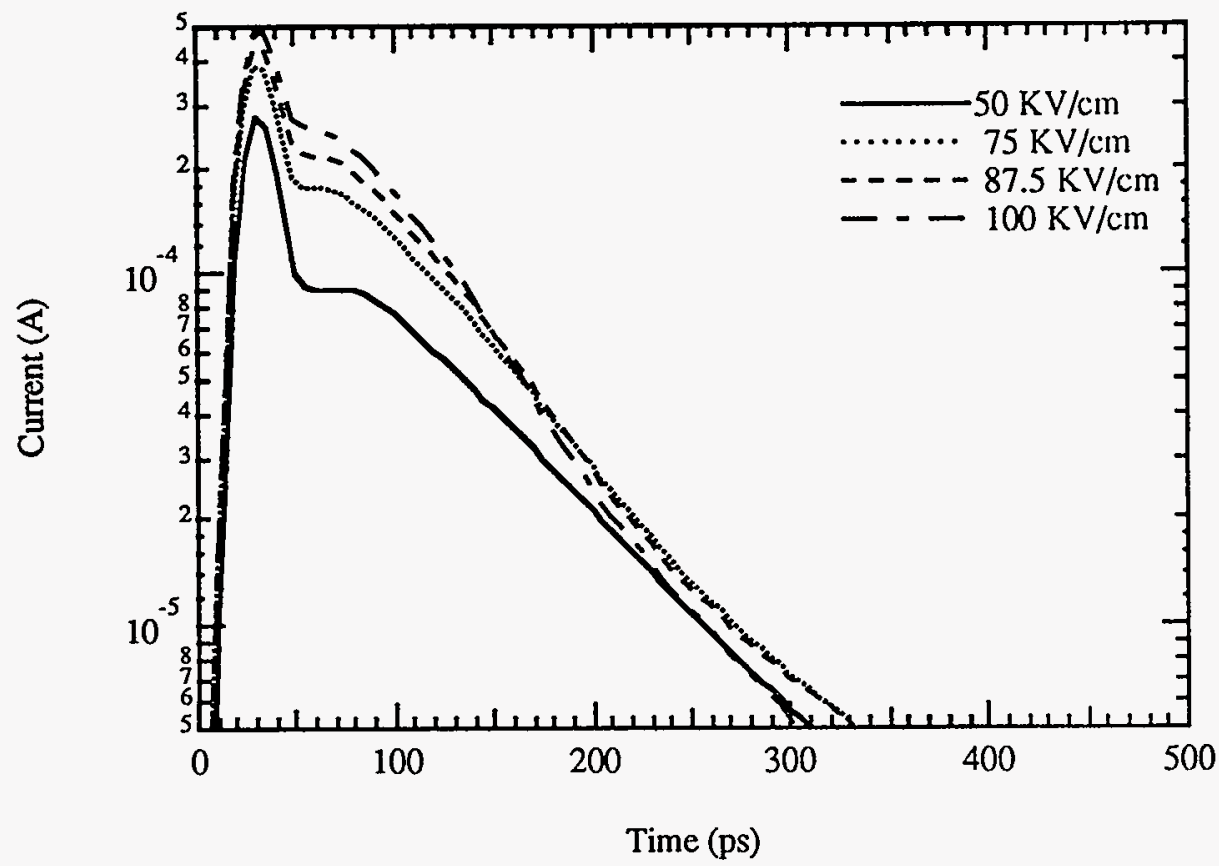

Figure 4.3.9: Calculated current transients as a function of applied electric field. All transients were smoothed with a Gaussian $1.3 \mathrm{ps}$ width representing the ion stopping time, followed by additional smoothing with a Gaussian of 18.6 ps width representing the system response function.

carriers reach the boundary of the detector by this time where they ultimately recombine and are completely removed. It is then expected that a greater fraction of carriers will reach the detector boundary at higher fields due to the larger drift velocities under these conditions. The reduced decay times for larger fields can then be ascribed to both trapping/recombination and higher carrier drift velocities.

Figures 4.3.10 - 4.3.13 show comparisons between measured and calculated current transients as a function of applied field and polarity. The material parameters used are listed in Table 4.3.2. The electron and hole capture cross sections listed above are about factors of 6 and 4 greater than those estimated by Pan [24], respectively, for 
A)

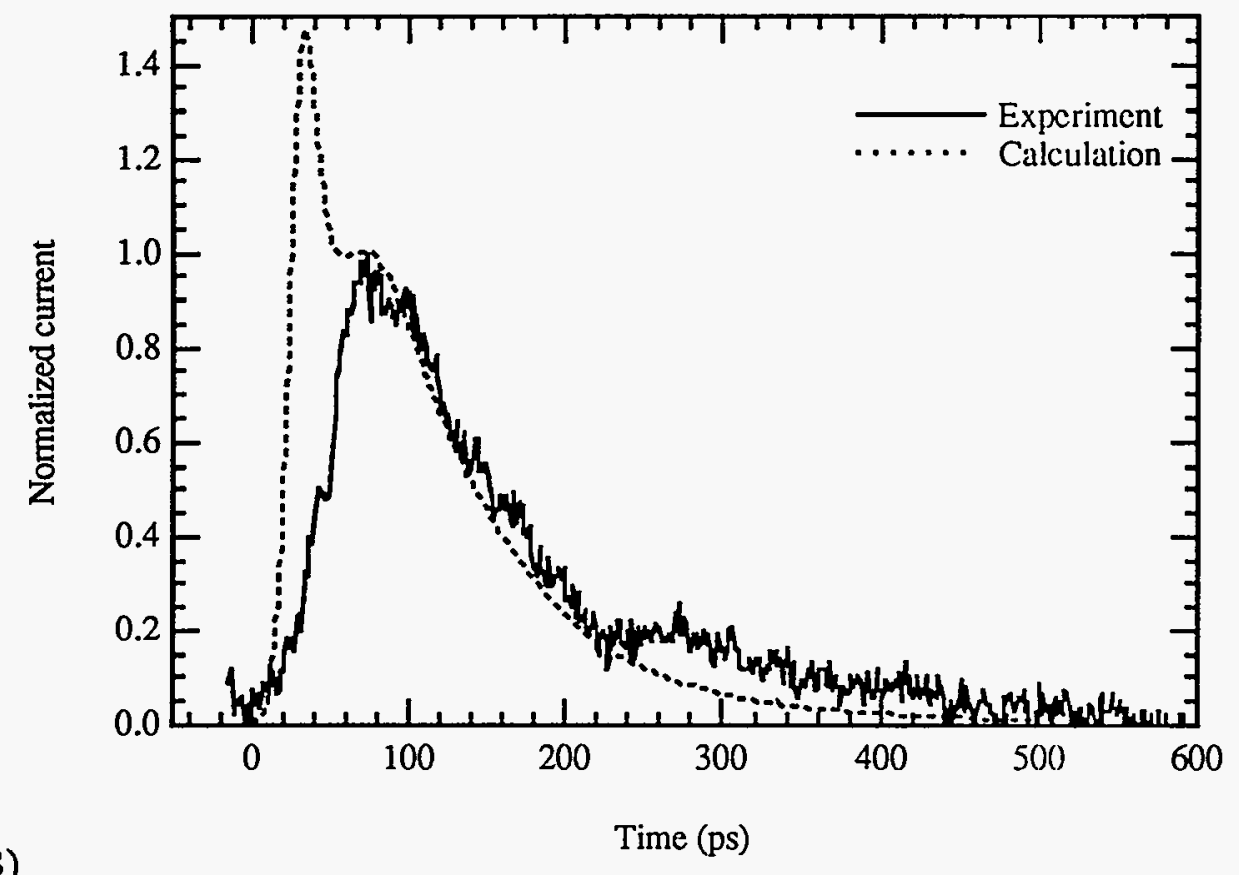

B)

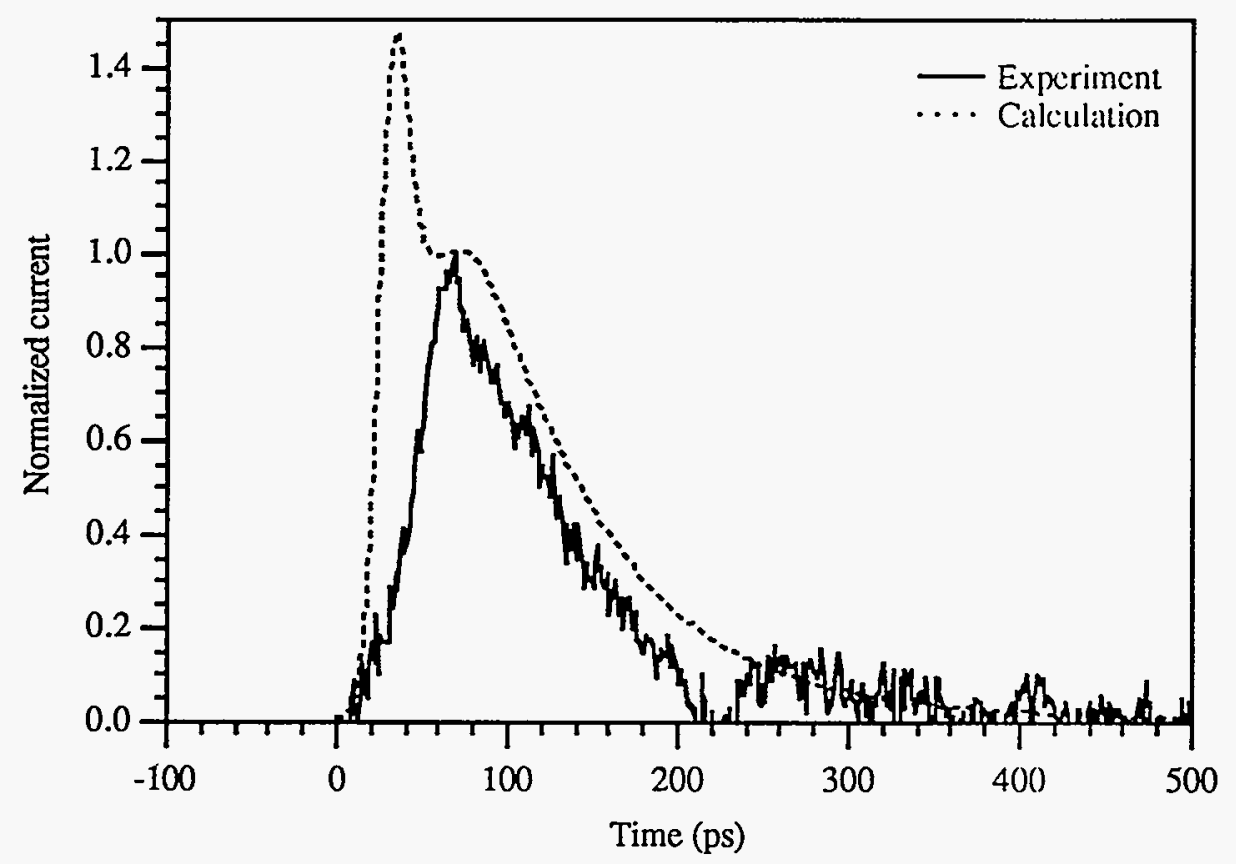

Figure 4.3.10: Comparison between calculated and measured transients for the stopping of $5 \mathrm{MeV} \mathrm{He}+$ ions in the detector; A) Applied field is $+50 \mathrm{KV} / \mathrm{cm}$.; B) Applied field is $-50 \mathrm{KV} / \mathrm{cm}$. 
A)

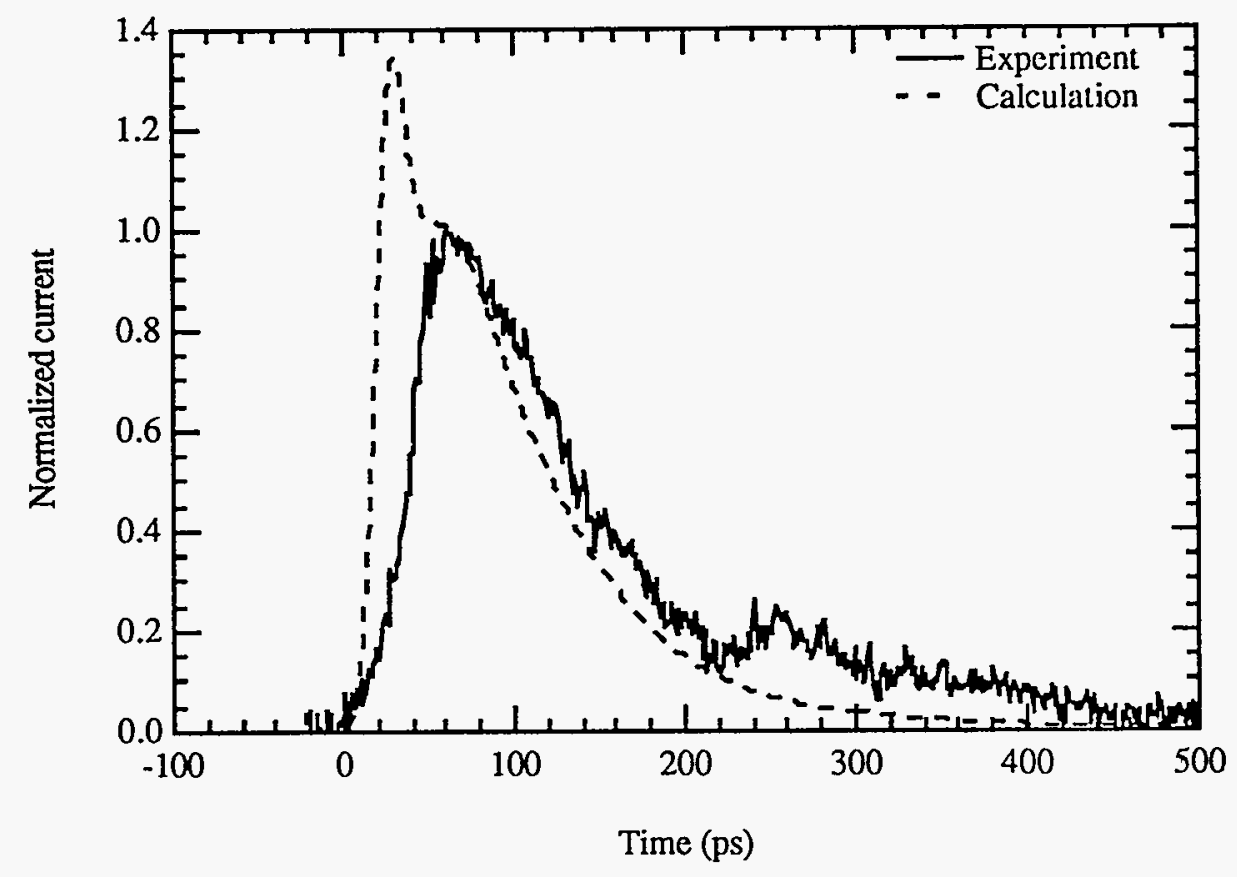

B)

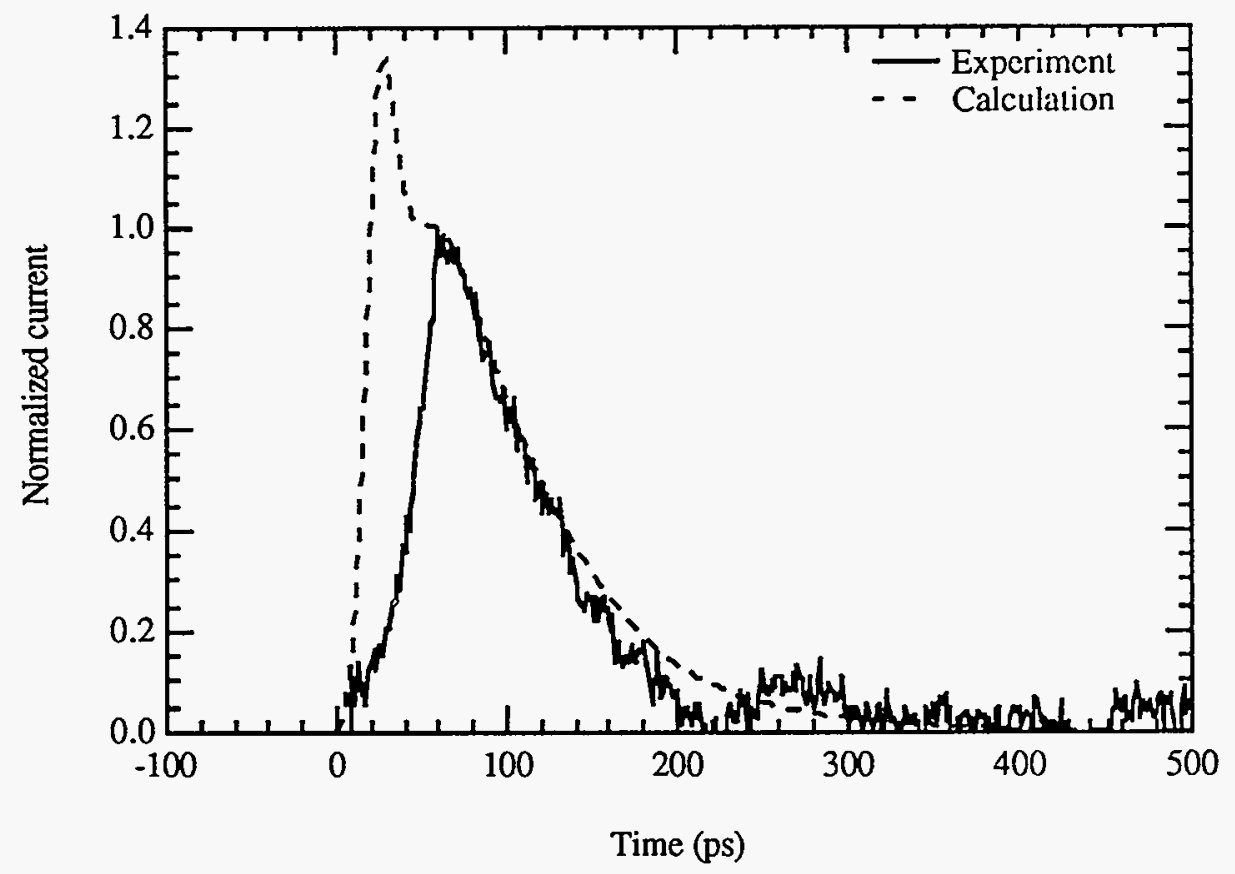

Figure 4.3.11: Comparison between calculated and measured transients for the stopping of $5 \mathrm{MeV} \mathrm{He} \mathrm{H}^{+}$ions in the detector; A) Applied field is $+75 \mathrm{KV} / \mathrm{cm}$.; B) Applied field is $-75 \mathrm{KV} / \mathrm{cm}$. 
A)
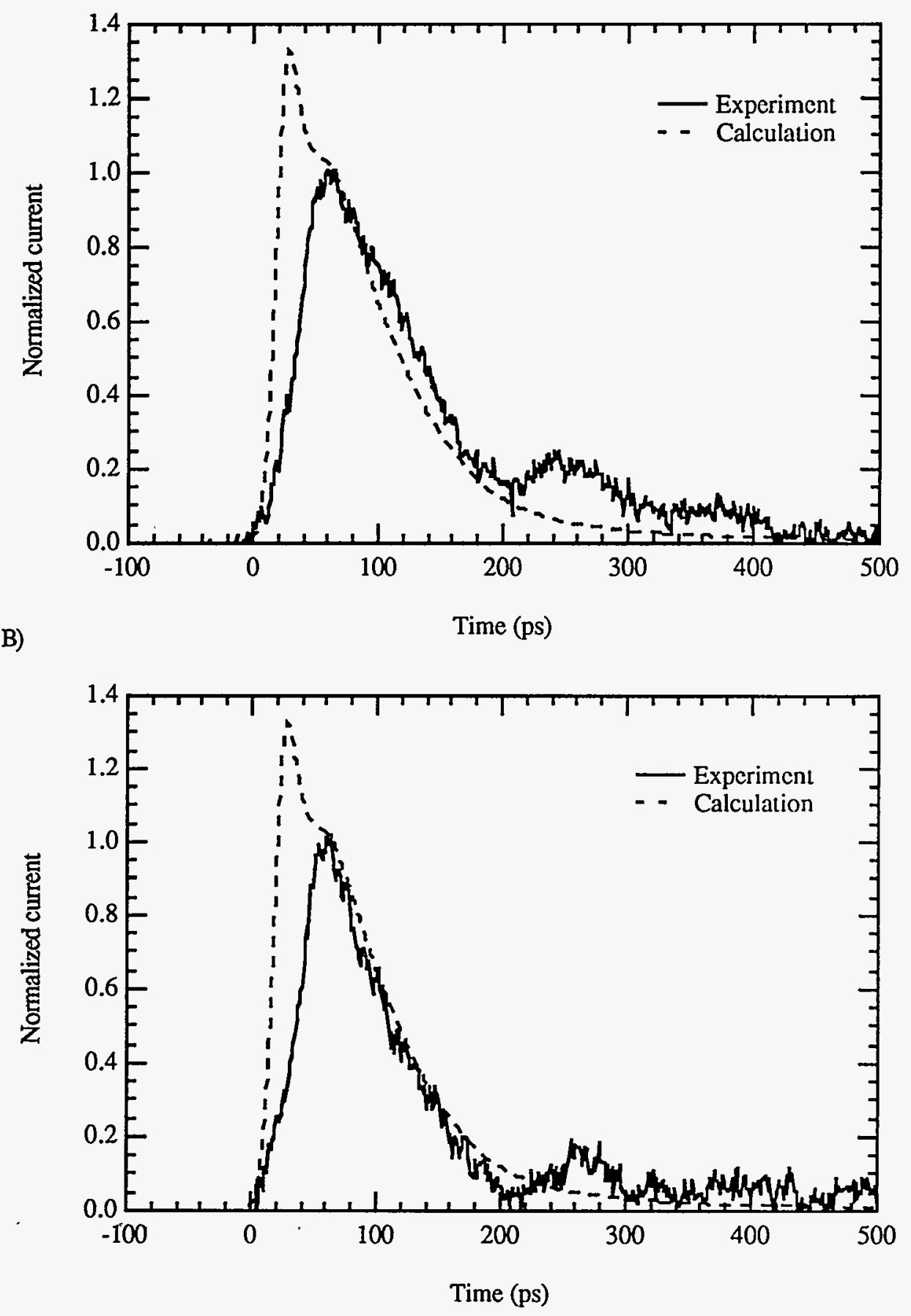

Figure 4.3.12: Comparison between calculated and measured transients for the stopping of $5 \mathrm{MeV} \mathrm{He}{ }^{+}$ions in the detector; A) Applied field is $+87.5 \mathrm{KV} / \mathrm{cm}$. ; B) Applied field is $-87.5 \mathrm{KV} / \mathrm{cm}$. 
A)

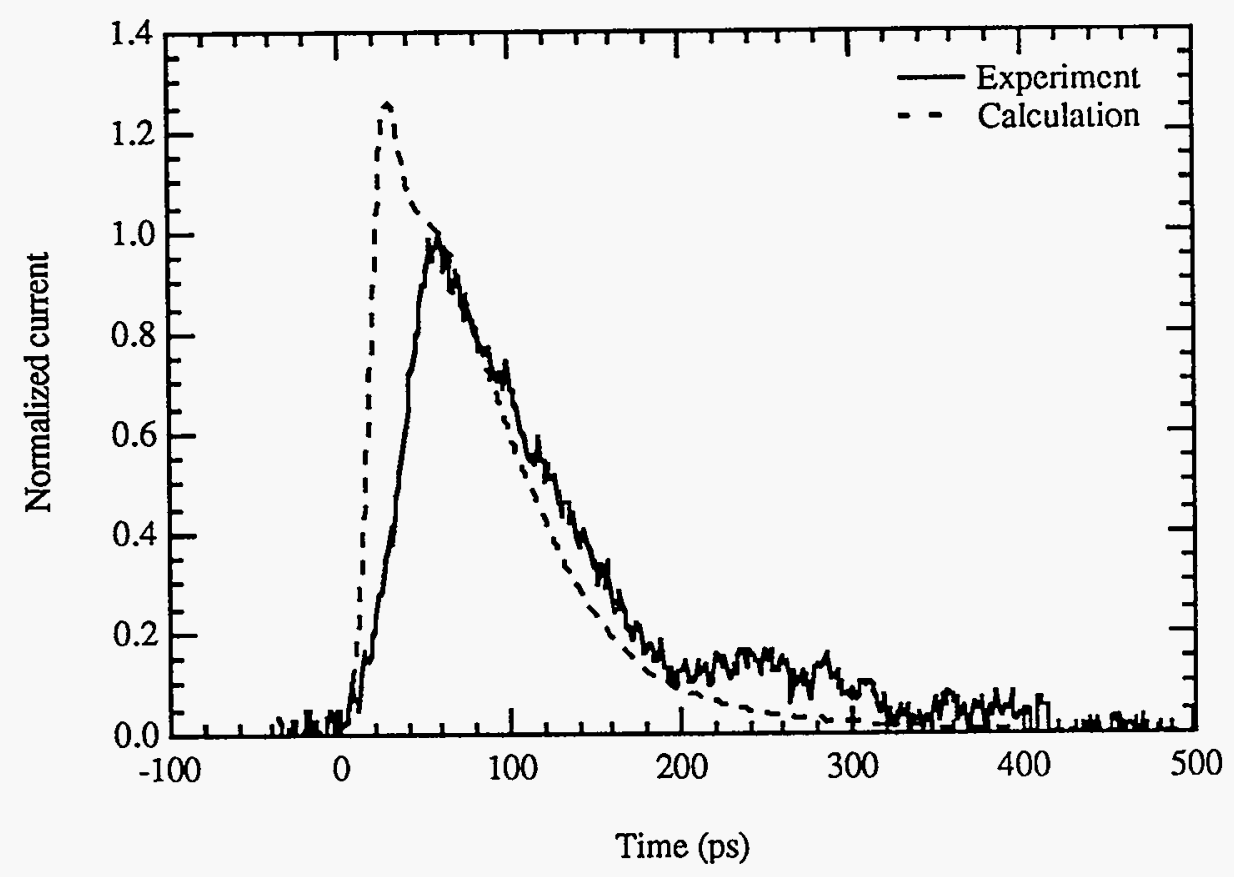

B)

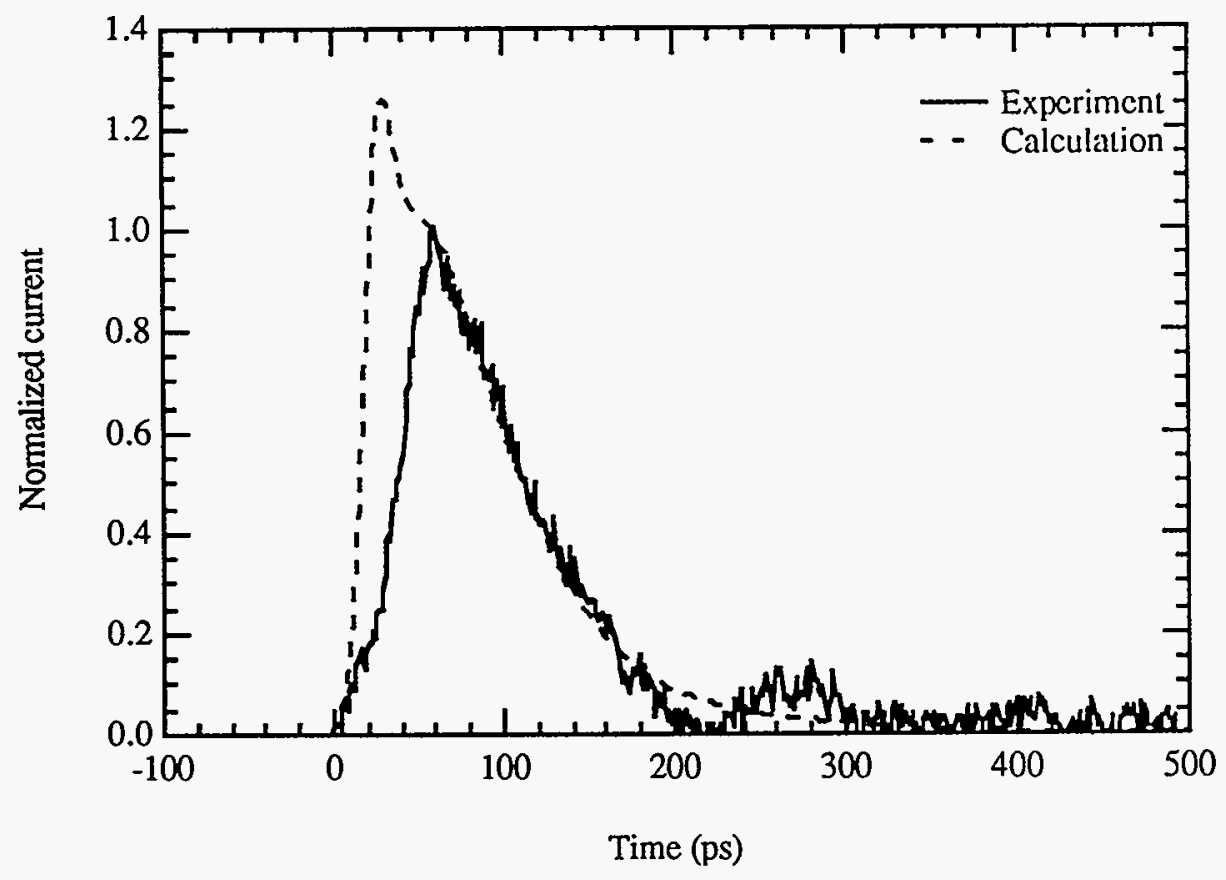

Figure 4.3.13: Comparison between calculated and measured transients for the stopping of $5 \mathrm{MeV} \mathrm{He}^{+}$ions in the detector; A) Applied field is $+100 \mathrm{KV} / \mathrm{cm}$; B) Applied field is $-100 \mathrm{KV} / \mathrm{cm}$. 
nitrogen. These differences are likely the result of additional trapping sites not included in the model.

The experimental transients have been individually normalized to their peak intensities and calculated transients have been individually normalized to the intensities of the second peak to simplify the comparisons of the shapes of the distribution functions.

Neglecting the peak beginning at about 200 ps in the experimental data that is due to reflections in the transmission line, the shapes of the calculated distributions for fields of $\pm 50, \pm 75$, and $\pm 88 \mathrm{kV} / \mathrm{cm}$ are in reasonable agreement with the decreasing part of the experimental transients. The calculated FWHM are about the same as those observed experimentally.

A quantitative comparison between the calculated and experimental transients is given in Table 4.3.3. The calculated peak currents represent the amplitude of the second peak in the calculated transients. All of the pulse parameters agree to within a factor of about 4 , and for the positive polarity data alone, the agreement is within a factor of 2.5 . For the reasons given above, comparisons will be focused on the data for the positive polarity pulses.

The total charge collected in the experiments is roughly accounted for in the calculations within a factor of 2.5 for the positive polarity. While it is difficult to draw hard conclusions, this may indicate that coupling between the transport of ions along the ion track does not produce grossly different transport characteristics from that obtained with the one-dimensional model. The larger discrepancy in the collected charge for the negative polarity is likely due to the additional trapping/recombination sites introduced through radiation damage. Because the current density is proportional to the product of free carrier density and their drift velocities, the discrepancy between measured and calculated peak currents can arise from differences between the assumed carrier mobilities and the initial carrier densities, and those of the real material and the actual densities. A more detailed comparison between the calculated and experimental peak 
Table 4.3.3: Comparison of parameters describing the calculated and experimental transients. In each column, the first number is the indicated ratio for the positive polarity pulse and the number in parentheses is the ratio for the pulse of negative polarity. The calculated peak currents are the peak amplitudes of the second peak in the calculated transients. The listed parameters are the applied electric field, the ratio of calculated peak current to measured, the ratio of calculated FWHM to measured and the ratio of calculated charge to measured.

\begin{tabular}{|cccc|}
\hline $\begin{array}{c}\text { Applied electric } \\
\text { field }(\mathrm{KV} / \mathrm{cm})\end{array}$ & $\frac{I_{\text {peak }}^{\text {calc }}}{I_{\text {peak }}^{\text {exp }}}$ & $\frac{F W H M_{\text {calc }}}{F W H M_{\text {exp }}}$ & $\frac{Q_{\text {coll }}^{\text {calc }}}{Q_{\text {coll }}^{\text {cxp }}}$ \\
\hline$+50(-50)$ & $1.08(1.52)$ & $0.93(1.34)$ & $1.57(2.75)$ \\
$+75(-75)$ & $1.69(2.13)$ & $0.87(1.15)$ & $1.72(2.45)$ \\
$+87.5(-87.5)$ & $1.82(2.32)$ & $0.88(1.15)$ & $2.42(3.37)$ \\
$+100(-100)$ & $2.32(2.73)$ & $0.82(1.00)$ & $2.42(3.66)$ \\
\hline
\end{tabular}




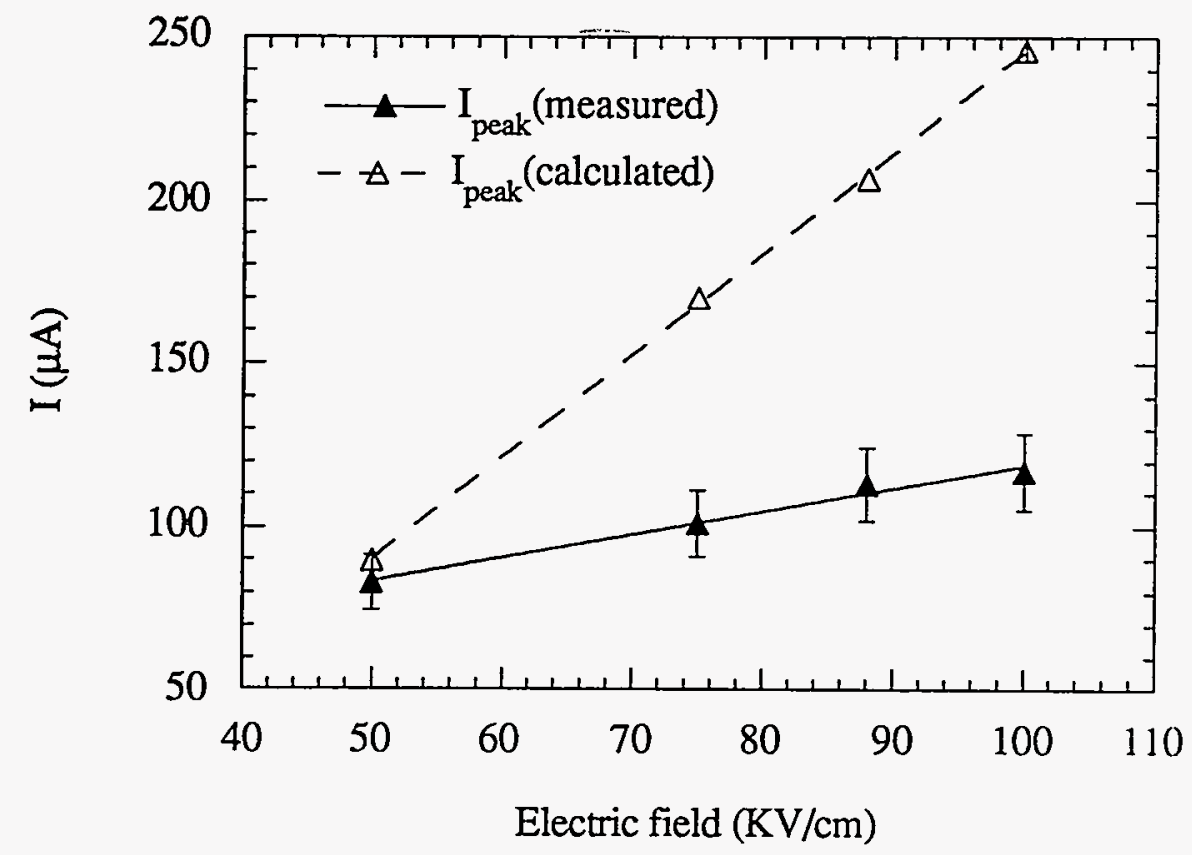

Figure 4.3.14: Calculated and measured peak currents as a function of electric field with bias polarity being positive. The lines through the data point are the linear fits with the correlation coefficient about 1.

currents is given in Fig. 4.3.14. Both sets of data show reasonably linear variations with the applied field, but the slope of the fit to the experimental data is considerably smaller than that predicted by the calculations.

Following the current transient experiments described here, the charge-integrated experiments with detector HE6.4 perform were performed using the experimental arrangement described in Sec. 2.2. It must be remembered that in the charge-integrated experiments the orientation of the ion track is parallel to the applied electric field whereas in the time-resolved current experiments the ion track is perpendicular to the field. A plot of the total charge collected per $\alpha$-particle versus applied electric field is given in Fig. 4.3.15. Also shown in the figure is a fit to the data with the charge collection model described in Sec. 3.2. As before, the fit assumes non-injecting contacts, a low-field electron-to-hole mobility ratio of 1.34 , and that the mean energy required to produce an 
electron-hole pair of $14.1 \mathrm{eV}$. The fit is quite good and comparable to the general quality of fits found with the other detectors discussed in Chapter 3. From the model fit, the magnitudes for the low-field electron and hole mobilities were estimated to be $124.7 \pm$ $31.6 \mathrm{~cm}^{2} / \mathrm{V}$-sec and $92.4 \pm 13.6 \mathrm{~cm}^{2} / \mathrm{V}$-sec, respectively. The low-field mobilities given above are smaller than those used for the one-dimensional transient model calculations by about a factor of 4 . The differences in the mobilities may be due to the radiation damage resulting from $\mathrm{He}$-ion bombardment during the current transient measurements. It is also possible that the difference in the estimated mobilities may be due, in part, to the collapse of the electric field and the non-uniform field distribution it produces which is not accounted for in the charge collection model.

In Fig. 4.3.16 is A plot of the charge collection efficiency, $\eta$, for this detector as a function of applied field. The charge collection efficiency varies from about $42 \%$ with an electric field of $10^{5} \mathrm{~V} / \mathrm{cm}$ to about $68 \%$ with an electric field of about $-2 \times 10^{5} \mathrm{~V} / \mathrm{cm}$. Taking a crude average of about $47 \%$, the collection efficiency is about a factor of 2.5 greater than that obtained during the current transient measurements at the same field.

It has been predicted by van Lint [25] and Nichols and van Lint [26] when a dense ionization track is present, it may be easier to collect charge with the ion track parallel to the applied electric field then when the track is perpendicular to the field. They speculated that the large space charge region that result with the applied field normal to the ion track causes a larger fraction of the charge in the center of the plasma column to be shielded in comparison to the case when the ion track is parallel to the field. The measurements with HE6.4 appears to support this speculation. 


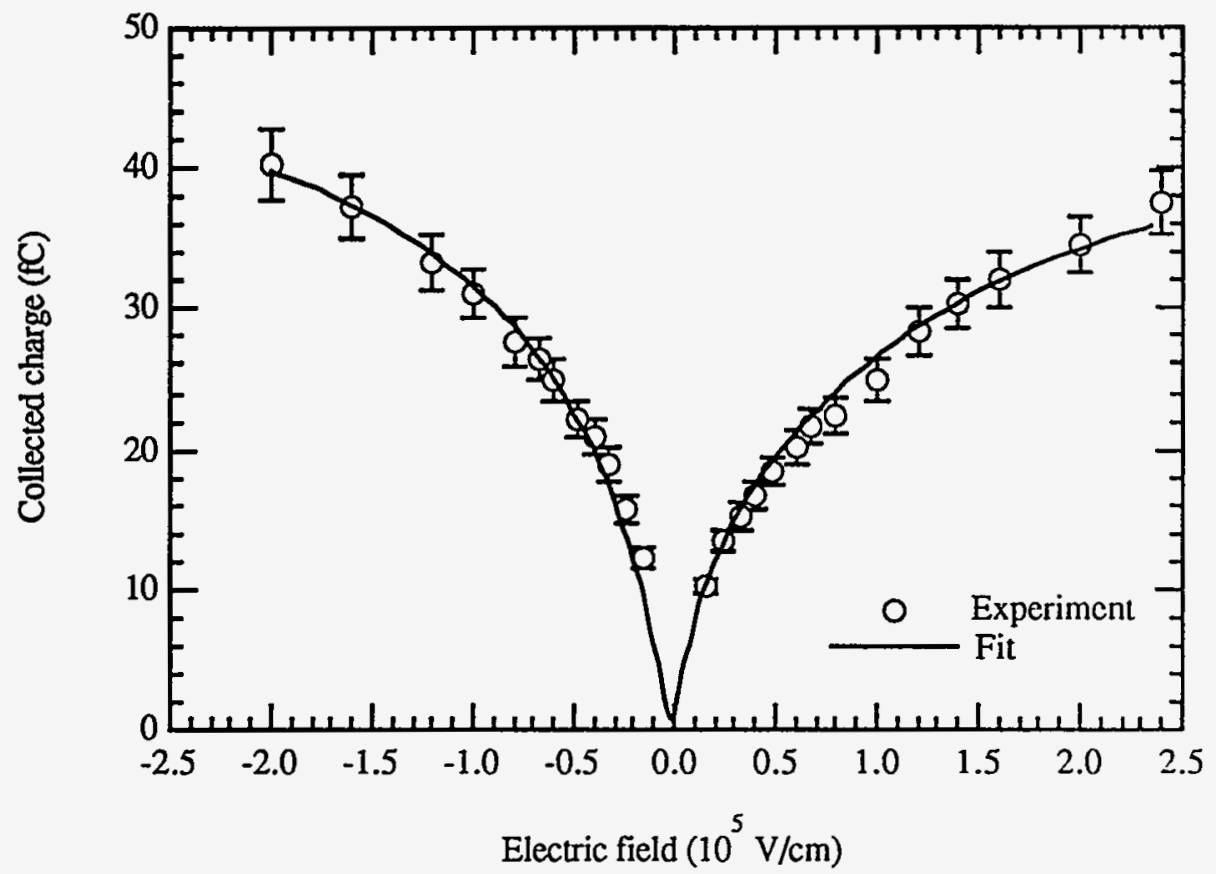

Figure 4.3.15: Charge collected from $241 \mathrm{Am} \alpha$-particles interacting with detector HE6.4 as a function of applied electric field. The curve shown represents a fit to the data with the model described in Chapter 3. 


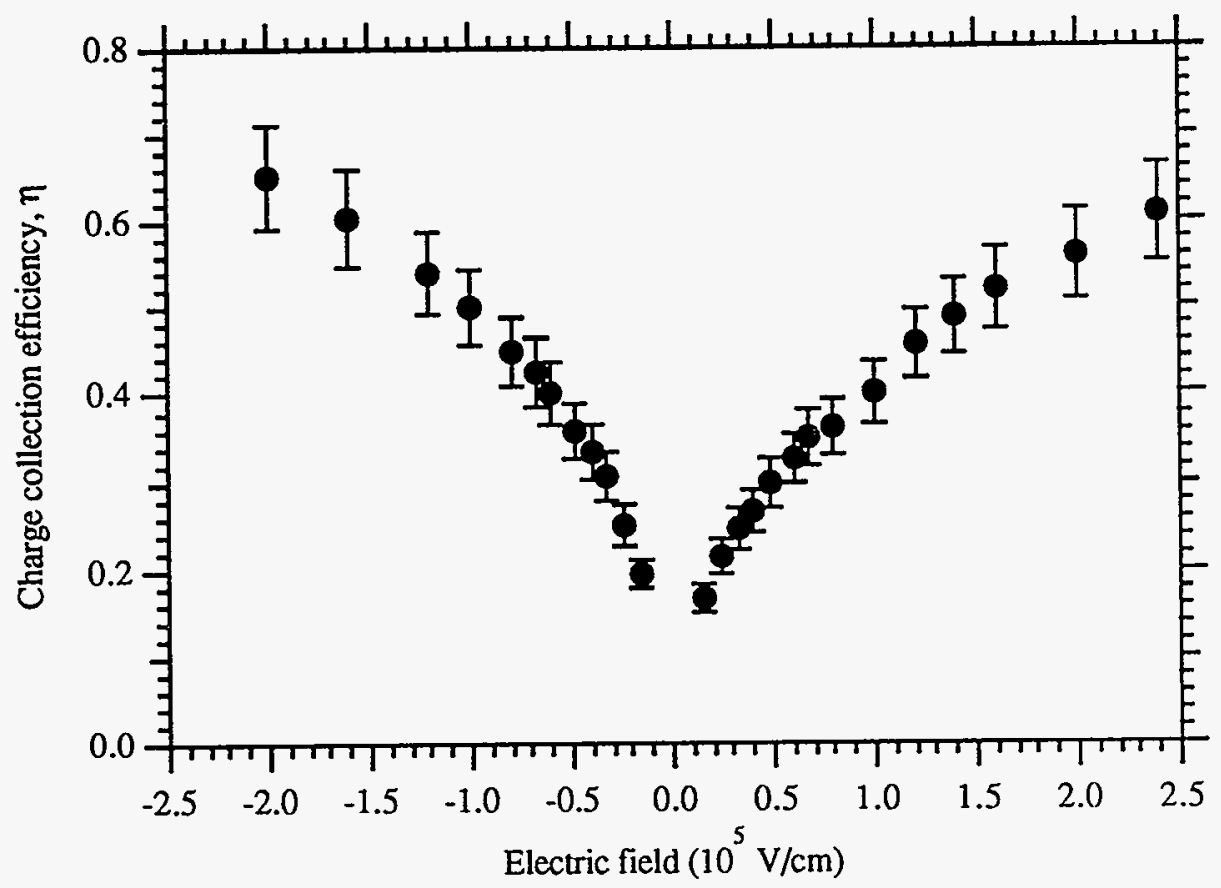

Figure 4.3.16: Charge collection efficiency, $\eta$, as a function of applied electric field for HE6.4 derived from excitation with $\alpha$-particles from ${ }^{241} \mathrm{Am}$. 


\subsection{2 $10-\mathrm{MeV} \mathrm{Si}^{3+}$-ion excitation}

Relative to the case of $5 \mathrm{MeV} \mathrm{He}^{+}$ions, the initial carrier densities produced by the $\mathrm{Si}$-ions is expected to be much larger. Based on the calculated mean range from the code TRIM [2] alone, the average density is expected to be larger by about an order of magnitude for cylindrically symmetric charge distributions of the same mean radius. This can be considered as the lower limit of the relative increase in carrier density because the maximum energy transfer to an electron in an elastic collision with a $10 \mathrm{MeV}$ $\mathrm{Si}^{+}$ion is significantly less than the transfer from interaction with a $5 \mathrm{MeV} \mathrm{He}^{+}$ion. Simple conservation of energy and momentum leads to the following expression for the maximum energy transfer, $T_{\max }$, when the target electron is unbound;

$$
T_{\max }=4 E_{i} \frac{m_{e}}{m_{i}}
$$

where $\mathrm{m}_{\mathrm{e}}=$ mass of electron

$$
\begin{aligned}
& m_{i}=\text { mass of ion } \\
& E_{i}=\text { kinetic energy of the ion }
\end{aligned}
$$

For $10 \mathrm{MeV} \mathrm{Si}$ ions, $\mathrm{T}_{\max }$ is about $0.8 \mathrm{keV}$ whereas for $5 \mathrm{MeV}$ He-ion, $\mathrm{T}_{\max }$ is about 2.7 $\mathrm{keV}$. Without detailed knowledge of the energy-dependent electron scattering cross sections in carbon, this energy difference cannot be used to calculate a corresponding difference in mean track radii. Nevertheless, it is clear that the expected width of the initial charge distribution for stopping of the $\mathrm{Si}$ ions is likely to be measurably smaller than that for the stopping of the He ions.

As a result of these considerations, calculations were performed with a range of track radii and corresponding charge densities obtained with the constraint that the total mean carrier production is constant. The track radii and corresponding peak charge densities used in the calculations are given in Table 4.3.4 and the material parameters are given in Table 4.3.5. 
Table 4.3.4: Peak carrier density, $n_{o}$, and corresponding ion track radius, $r$.

\begin{tabular}{|cc|}
\hline $\mathrm{r}(\mu \mathrm{m})$ & $n_{o}\left(10^{16} \mathrm{~cm}^{-3}\right)$ \\
\hline 1 & 24.9 \\
1.5 & 11.1 \\
2 & 6.2 \\
3 & 2.8 \\
\hline
\end{tabular}

Table 4.3.5: Materials parameters used in model calculations for $10 \mathrm{MeV} \mathrm{Si}^{3+}$-ion excitation. In order, the columns show the low-field electron mobility $\left(\mu_{o e}\right)$, the low-field electron mobility $\left(\mu_{\mathrm{oh}}\right)$, the electron capture cross section $\left(\sigma_{\mathrm{e}}\right)$, the electron capture cross section $\left(\sigma_{\mathrm{h}}\right)$, and the total nitrogen concentration $\left(\mathrm{N}_{\mathrm{T}}\right)$.

\begin{tabular}{|ccccc|}
\hline $\begin{array}{c}\mu_{\mathrm{oe}} \\
\left(\mathrm{cm}^{2} / \mathrm{V}-\mathrm{sec}\right)\end{array}$ & $\begin{array}{c}\mu_{\mathrm{oh}} \\
\left(\mathrm{cm}^{2} / \mathrm{V}-\mathrm{sec}\right)\end{array}$ & $\begin{array}{c}\sigma_{\mathrm{e}} \\
\left(\mathrm{cm}^{2}\right)\end{array}$ & $\begin{array}{c}\sigma_{\mathrm{h}} \\
\left(\mathrm{cm}^{2}\right)\end{array}$ & $\begin{array}{c}N_{\mathrm{T}} \\
\left(\mathrm{cm}^{-3}\right)\end{array}$ \\
\hline 1500 & 1119 & $2 \times 10^{-13}$ & $5 \times 10^{-16}$ & $2 \times 10^{18}$ \\
\hline
\end{tabular}

In Fig. 4.3.17, the experimental current transient measured with detector Si6.9 at an applied field of $37.5 \mathrm{KV} / \mathrm{cm}$ is shown along with calculated transients for a range of track radii. Because the average stopping time of a $10 \mathrm{MeV} \mathrm{Si}{ }^{+}$ion is less than $0.5 \mathrm{ps,}$ the calculated transients were smoothed only with a Gaussian of $18.6 \mathrm{ps}$ FWHM representing the response of the recording system. In comparison to the case of $5 \mathrm{MeV}$ $\mathrm{He}^{+}$ions, the difference between calculated and measured transients are much larger. Regardless of the assumed track radius, the calculated currents are dominated by the prominent peak from dielectric collapse followed by a relatively slow decrease in current with time. The experimental data display only a broad, slowly decreasing peak which has 


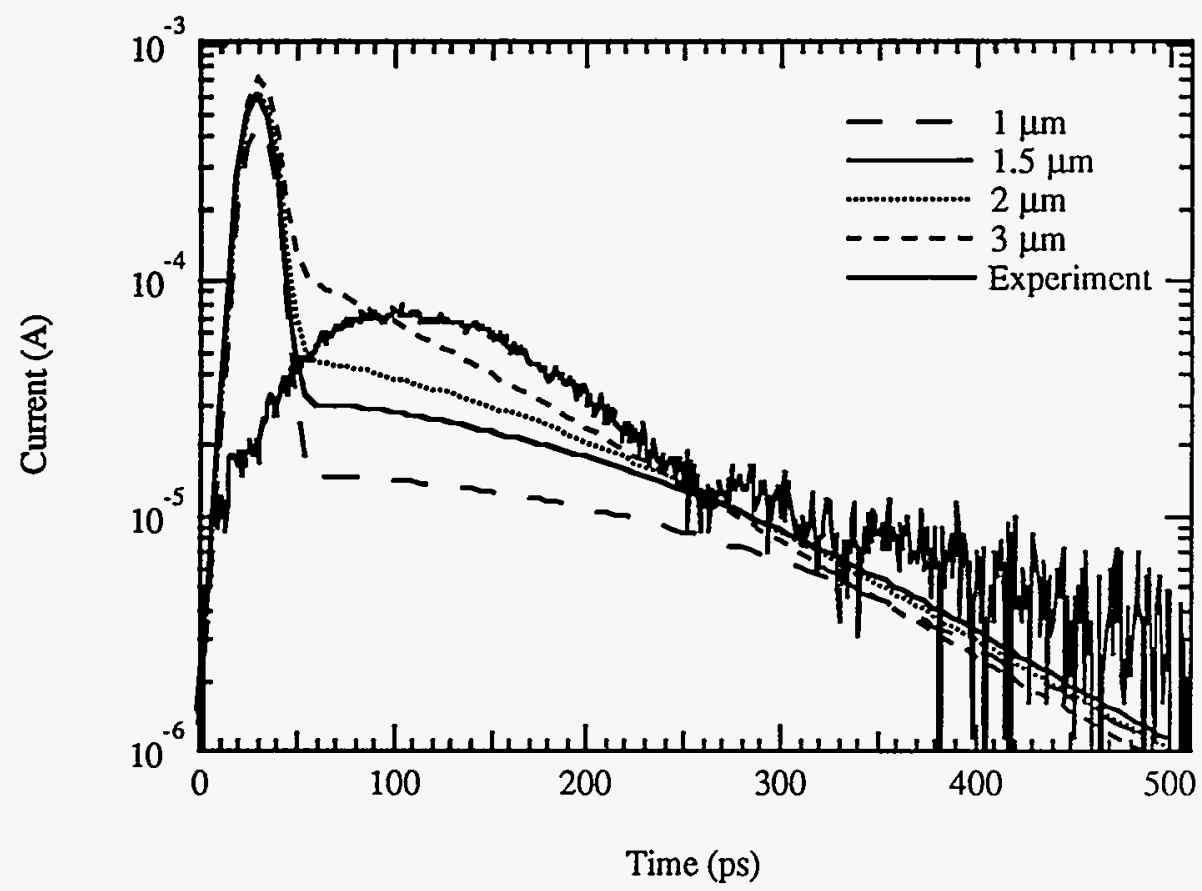

Figure 4.3.17: Comparison between measured and calculated response of the detector as a function of track radius with all other computational parameters being constant. listed in Table 4.3.4 and the transient

a longer rise time then could be ascribed with the long-time response of the calculated transients.

As discussed above, the magnitude of the calculated peak due to dielectric collapse is dependent upon the assumption that all charges are present initially, and it is likely to be strongly affected by the neglect of coupling between transport of ions at different locations along the trajectory of a $\mathrm{Si}^{+}$ion. If this part of the predicted response exists, the experimental data suggest that its magnitude can be no more than about $10 \%$ of that calculated.

Because of the poor agreement between calculated and measured transients the total nitrogen concentration was increased to $3 \times 10^{18} \mathrm{~cm}^{-3}$ with all other computational parameters shown in Table 4.3 .5 held constant. The track radius was taken as $1.5 \mu \mathrm{m}$. The calculated current transient is shown along with the experimental data and the 
transient with track radius of $1.5 \mu \mathrm{m}$ from Fig. 4.3.17 in Fig. 4.3.18. Very little improvement in the fit of the calculations to experiment is seen. It is unlikely that a further increase in the density of traps for holes alone will produce shorter carrier lifetimes as well as permit a reasonable magnitude for the calculated current.

The effect of carrier mobilities was tested by a calculation where they were reduced by a factor of 3 as compared to the ones listed in Table 4.3.5. All other parameters were held constant. Fig. 4.3.19 shows the comparison between measured and calculated transients. There is a significant improvement in the definition of the early part of the broad current peak, but the long decay time in the calculation persists.

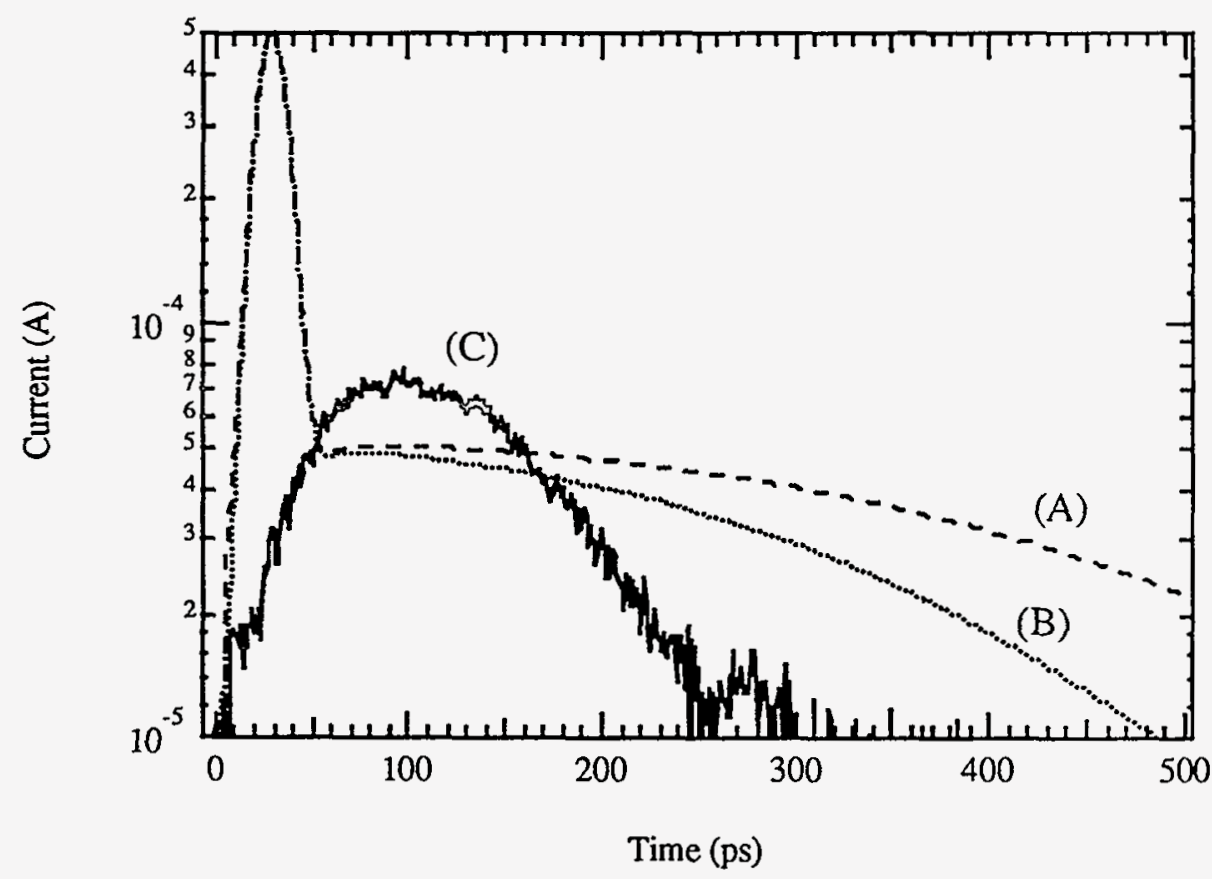

0

Figure 4.3.18: Comparison between measured and calculated transient currents of the detector with the track radius set at $1.5 \mu \mathrm{m}$; (A) calculated transient using material parameter listed in Table 4.3.4; (B) increased nitrogen concentration, $\mathrm{N}_{\mathrm{T}}$, to $3 \times 10^{18} \mathrm{~cm}^{-3}$; (C) measured current transient. Applied electric field is $37.5 \mathrm{KV} / \mathrm{cm}$. 


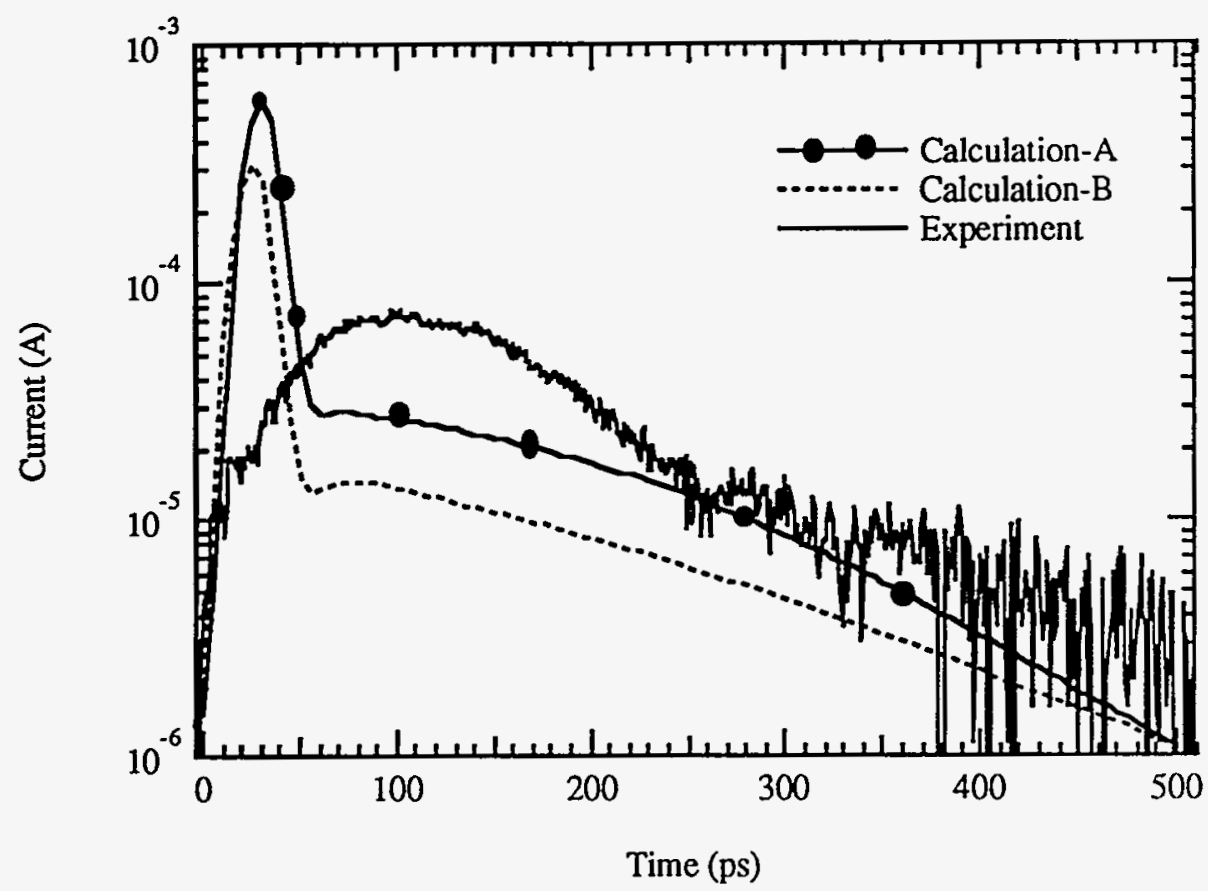

Figure 4.3.19: Comparison between measured and calculated response of the detector with the track radius set at $1.5 \mu \mathrm{m}$; (Calculation-A) carrier mobilities were the same as the ones found in Table 4.3.4; (Calculation-B) carrier mobilities were reduced by a factor of 3 .

The rest of the calculations in this section will be done using all of the calculational parameters listed in Table 4.3.5 except the values of the carrier mobilities; they will be reduced by a factor of 3 . Furthermore, the first $15 \mathrm{ps}$ of the instantaneous current density profile will be approximated with an exponential function as previously discussed in Sec. 4.3.1.

Figures 4.3.20-4.3.23 show the comparison between measured and calculated transient with the applied field in the range $37.5-100 \mathrm{KV} / \mathrm{cm}$ for both bias polarities. As it was mentioned previously, the first 15 ps of the transient was fitted an exponential function and then smoothed with a 18.6-ps Gaussian response function. For all fields, the disagreement in shape between measured and calculated is quite large. All calculated 
A)

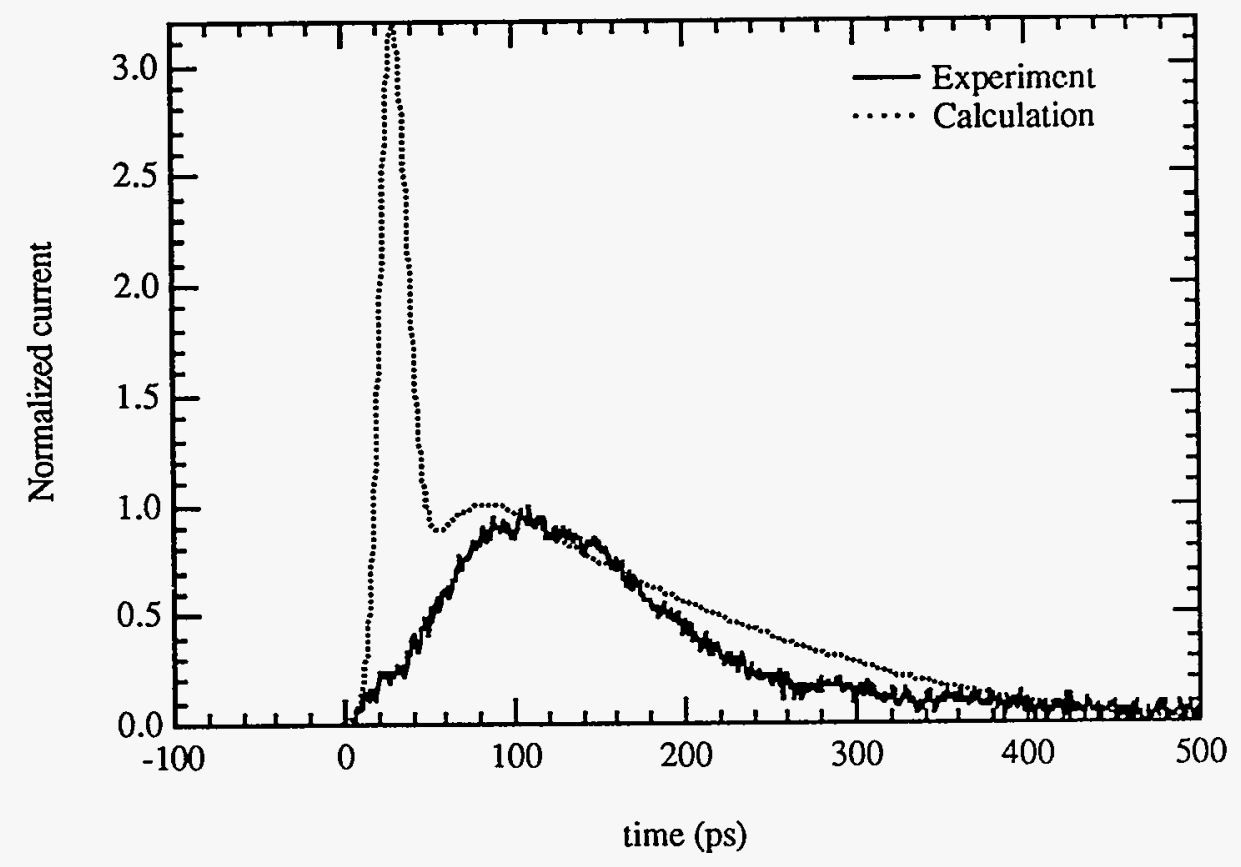

B)

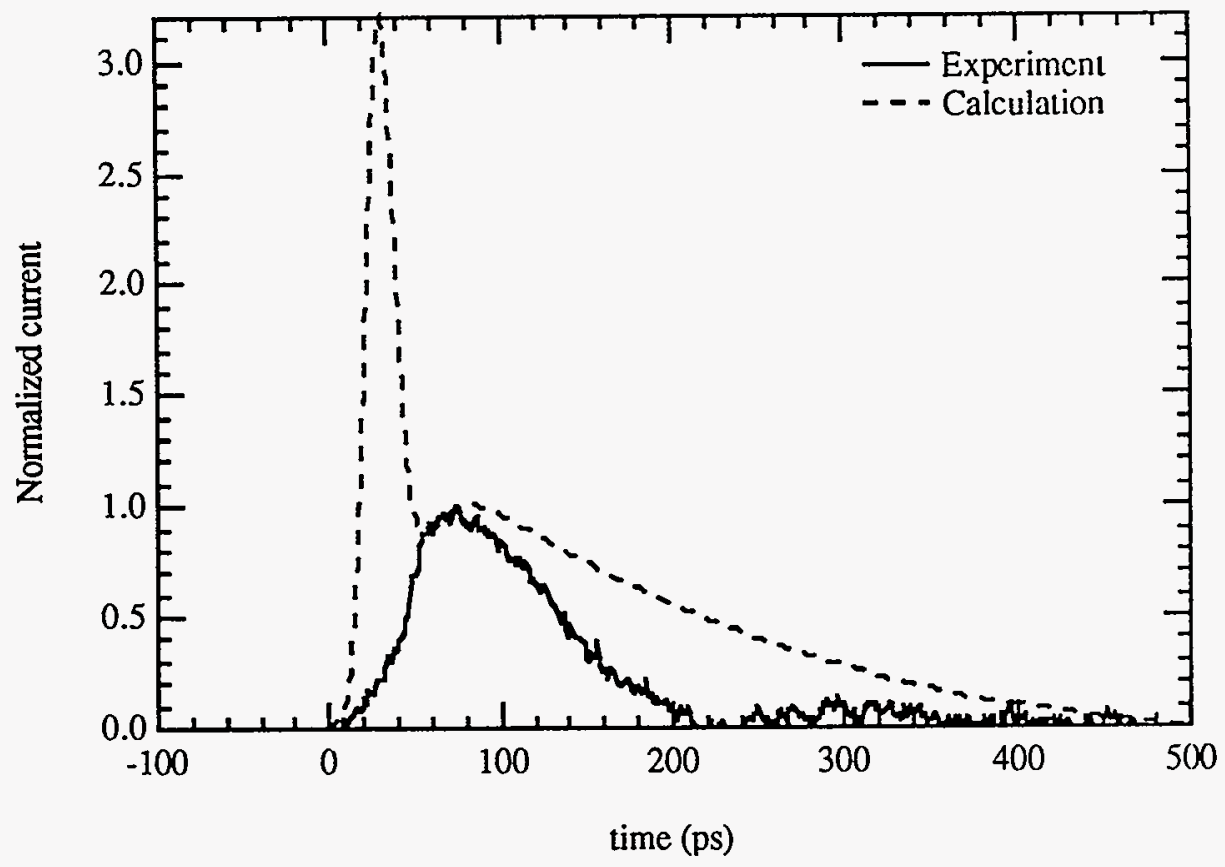

Figure 4.3.20: Comparison between calculated and measured transients for the stopping of $10 \mathrm{MeV} \mathrm{Si}^{3+}$ ions in the detector; A) Applied field is $+37.5 \mathrm{KV} / \mathrm{cm}$.; B) Applied field is $-37.5 \mathrm{KV} / \mathrm{cm}$. 
A)

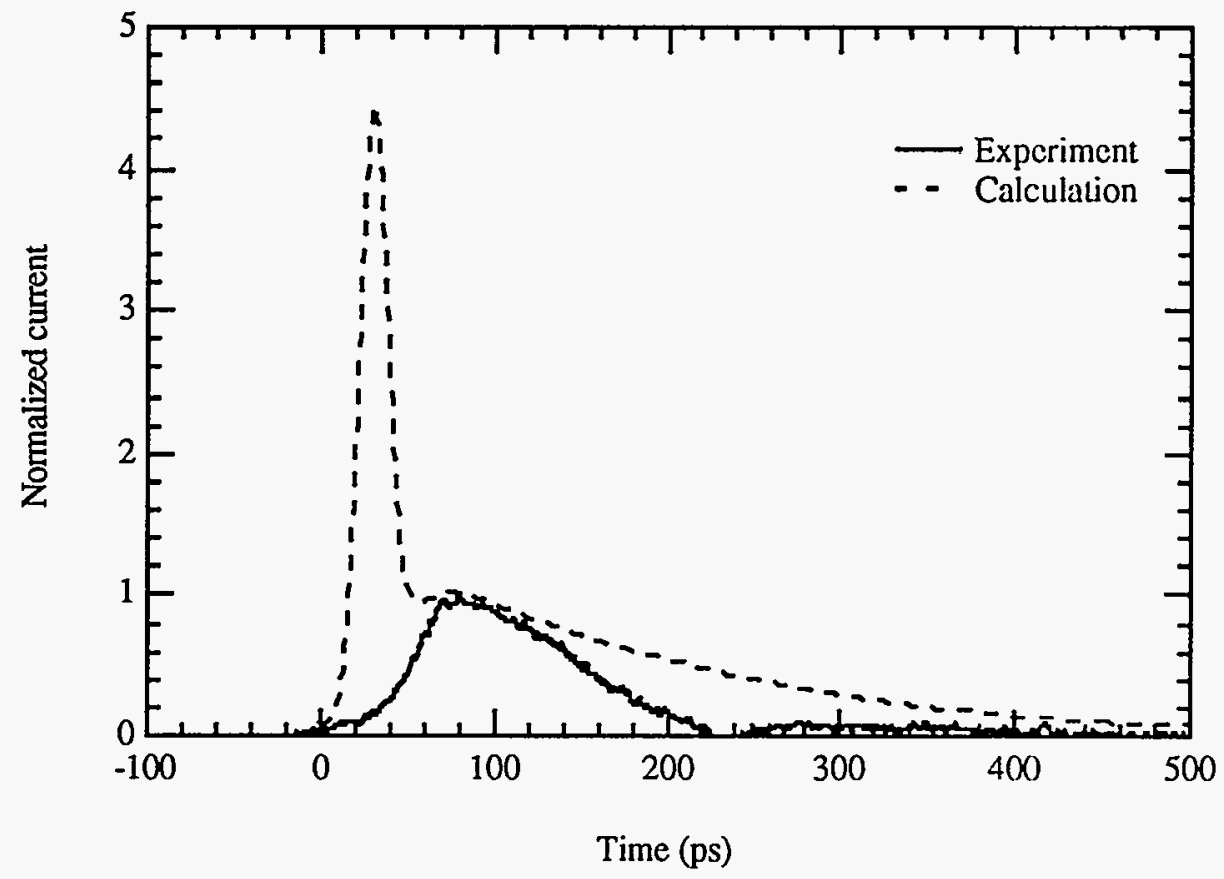

B)

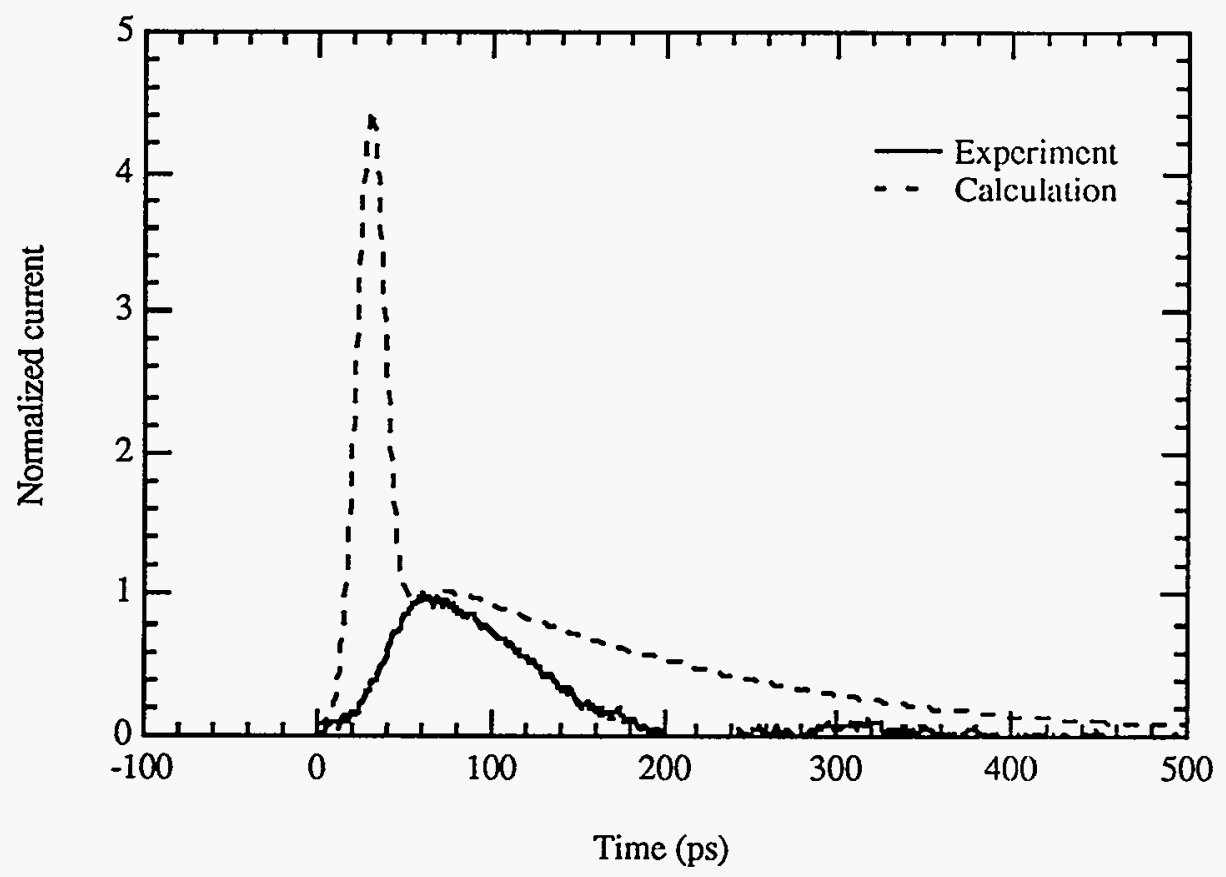

Figure 4.3.21: Comparison between calculated and measured transients for the stopping of $10 \mathrm{MeV} \mathrm{Si}^{3+}$ ions in the detector; A) Applied field is $+50 \mathrm{KV} / \mathrm{cm}$.; B) Applied field is $-50 \mathrm{KV} / \mathrm{cm}$. 
A)

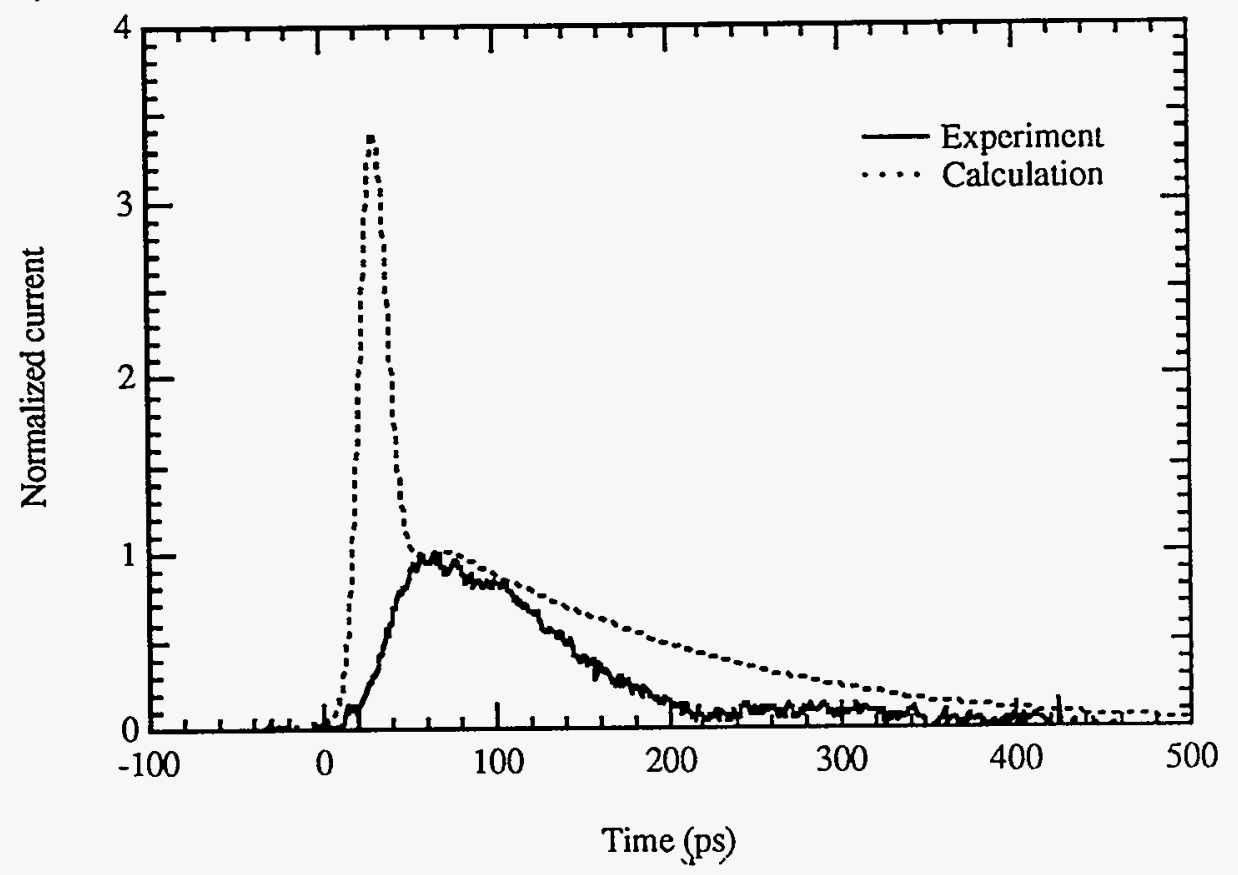

B)

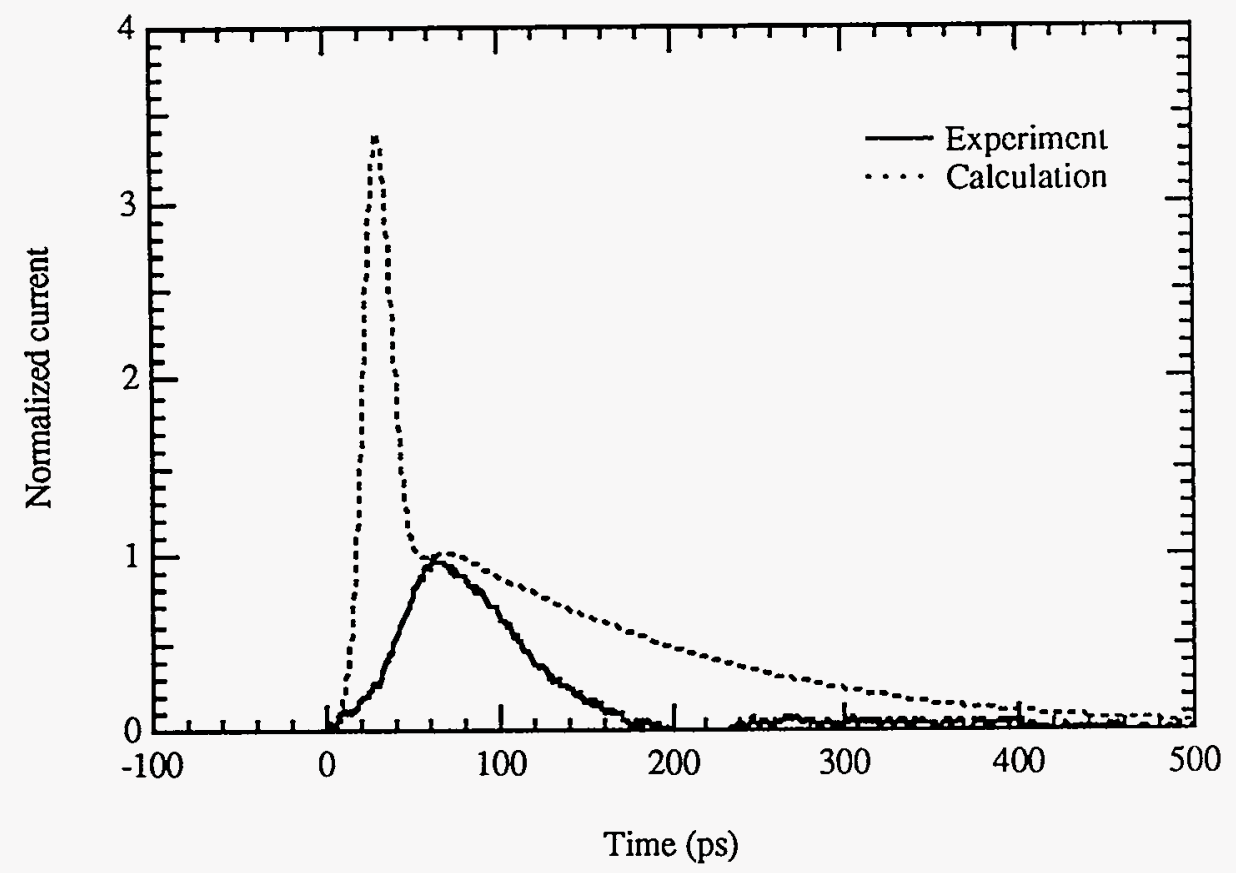

Figure 4.3.22: Comparison between calculated and measured transients for the stopping of $10 \mathrm{MeV} \mathrm{Si}^{3+}$ ions in the detector; A) Applied field is $+75 \mathrm{KV} / \mathrm{cm}$; B) Applied field is $-75 \mathrm{KV} / \mathrm{cm}$. 
A)

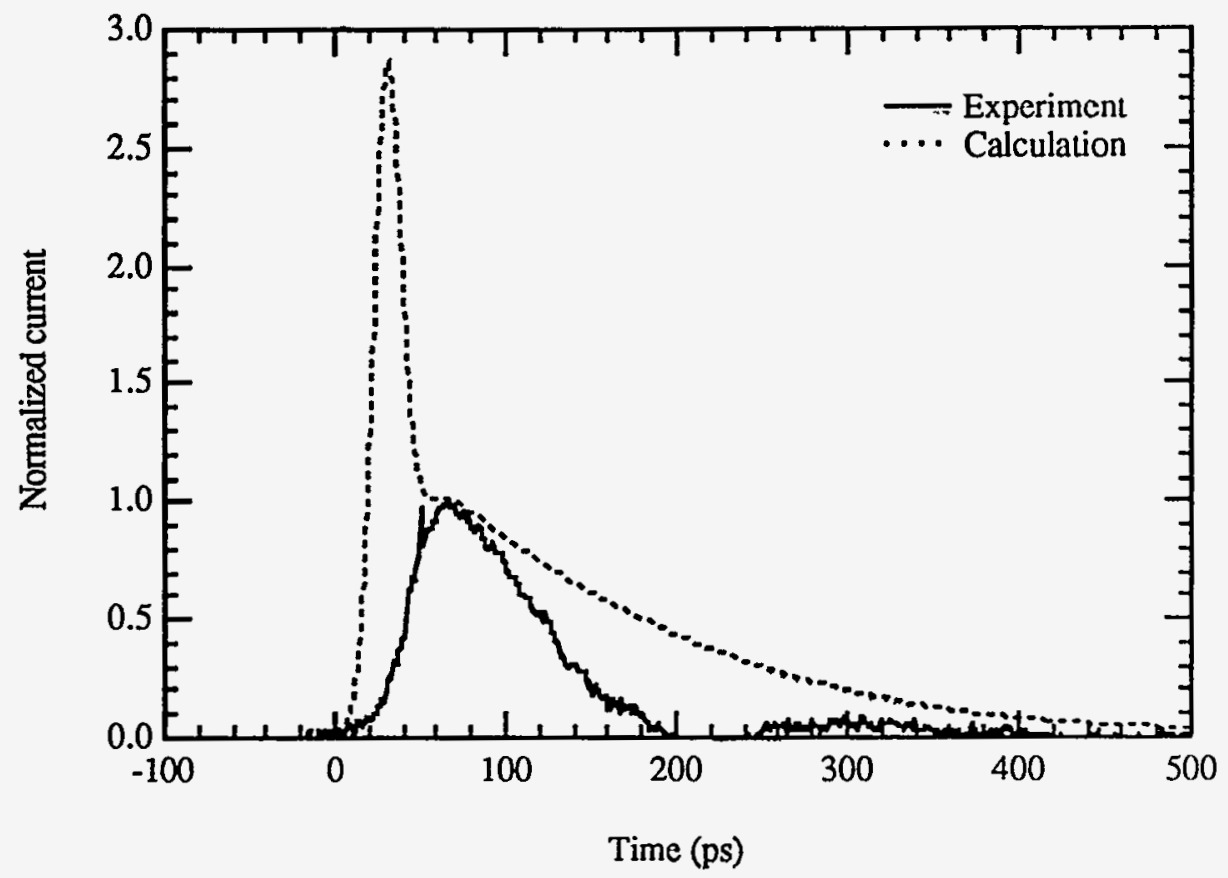

B)

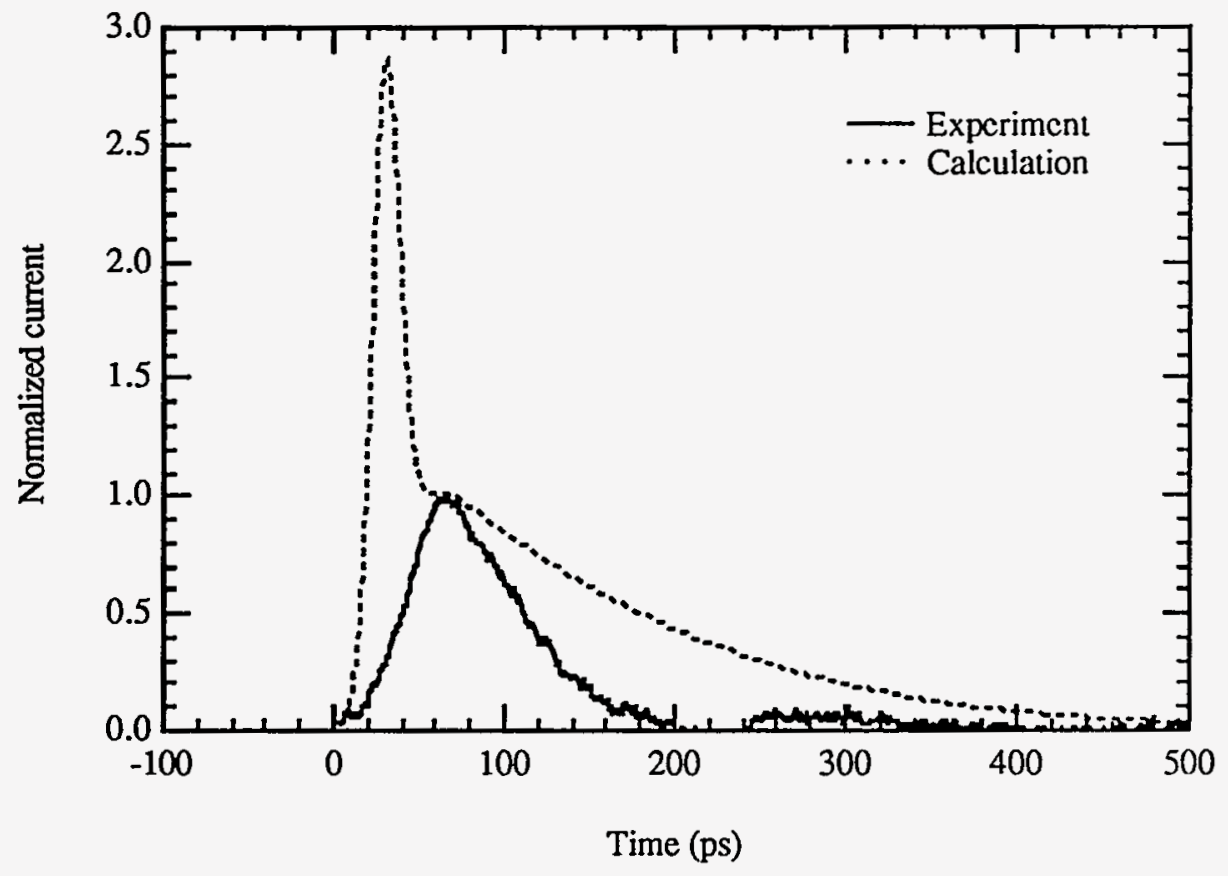

Figure 4.3.23: Comparison between calculated and measured transients for the stopping of $10 \mathrm{MeV} \mathrm{Si}^{3+}$ ions in the detector; A) Applied field is $+100 \mathrm{KV} / \mathrm{cm}$; B) Applied field is $-100 \mathrm{KV} / \mathrm{cm}$. 
transients show much large pulse-decay times as compared to experiment. This indicates that additional trapping/recombination centers are present in addition to nitrogen and must play a more critical role in defining the observation of the transient than in the case for He excitation. It is quite likely that the one trap model used here is a significant source of the disagreement between the calculated and experimental current transients.

A number of factors must be considered to account for the disagreement between the shapes of the calculated and measured transients. First, reference to Fig. 4.1.2 shows that the peak stopping power of $10 \mathrm{MeV}$ Si ions in diamond occurs at the entrance into the material. The shape of the stopping power distribution implies that the carrier density distribution may be nearly spherical in shape as compared to long and narrow cylindrical shape for $5 \mathrm{MeV}$ He excitation. Therefore, any error in treating the initial carrier density distribution as cylindrical with radius small compared to the axial extent of the track will be greatly magnified with $\mathrm{Si}$ as a result.

Second, the extent of range and angular straggling of the $\mathrm{Si}$ ions is much greater than for the $\mathrm{He}$ ions as seen in Fig. 4.3.26. On the average, the tracks of the He ions are quite linear and the ratio of the maximum deviation from an average linear track, about $3 \mu \mathrm{m}$, is about $28 \%$ of the mean range itself. For the Si-ions, however, the deviation is about $1.2 \mu \mathrm{m}$, which is about $47 \%$ of the mean range of the ions., and the track distribution is more nearly conical in character. In fact, a significant fraction of the Si ion track is parallel to the electric field, and it is expected that charge collection under this geometry will be markedly different from the case of tracks normal to the electric field $[20,21]$. To illustrate such differences, Fig. 4.3.27 shows calculated current transients for Si-ion tracks parallel to the applied field (Fig. 4.3.27-A) and normal to the applied electric field (Fig. 4.3.27B). The computational parameters given in Table 4.3.6 were used except, again, with the reduction of the carrier mobilities by a factor of 3 . Both the total charge collected as well as the temporal variation of the current are quite different for the two geometries. It seems clear that a simple one-dimensional model is a poor 
approximation to the case of short, high-density tracks with significant range and angular straggling. 
(A)

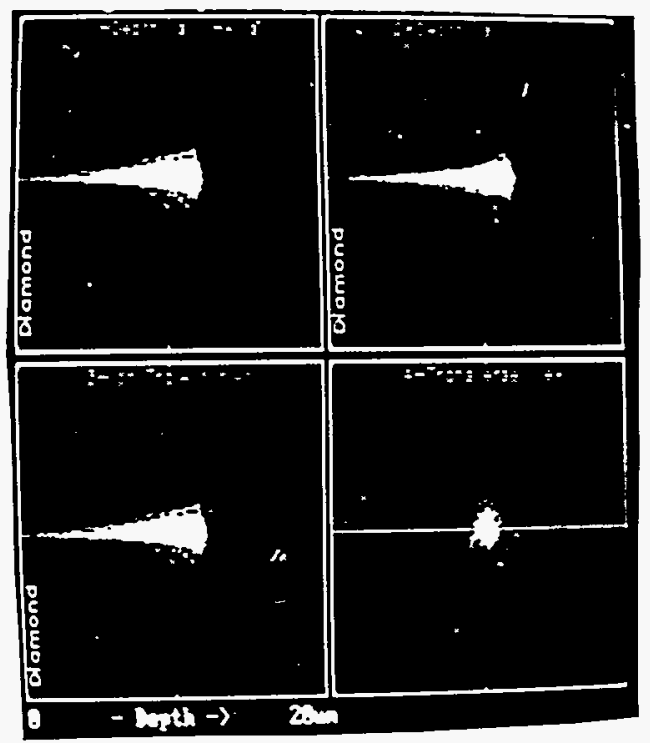

(B)

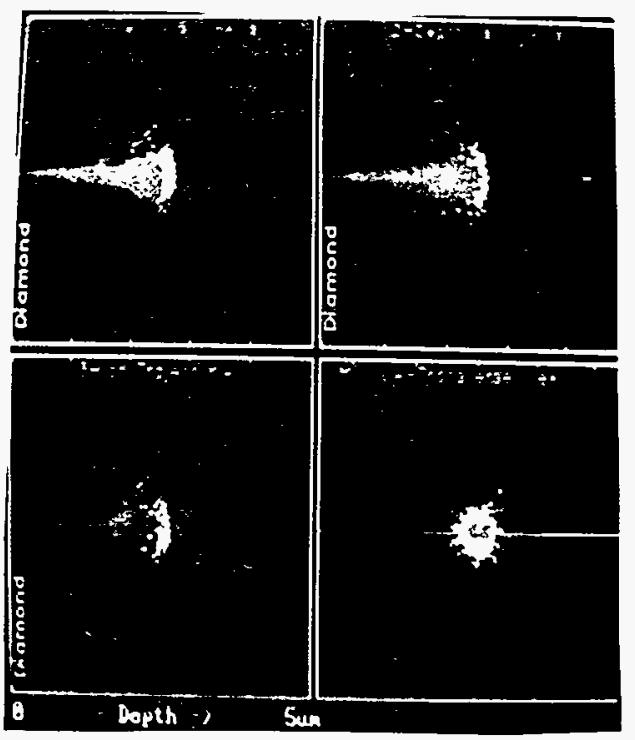

Figure 4.3.24: Ion track orientation distribution as calculated with TRIM; (A) 5 MeV He; (B) $10 \mathrm{MeV} \mathrm{Si.}$ 


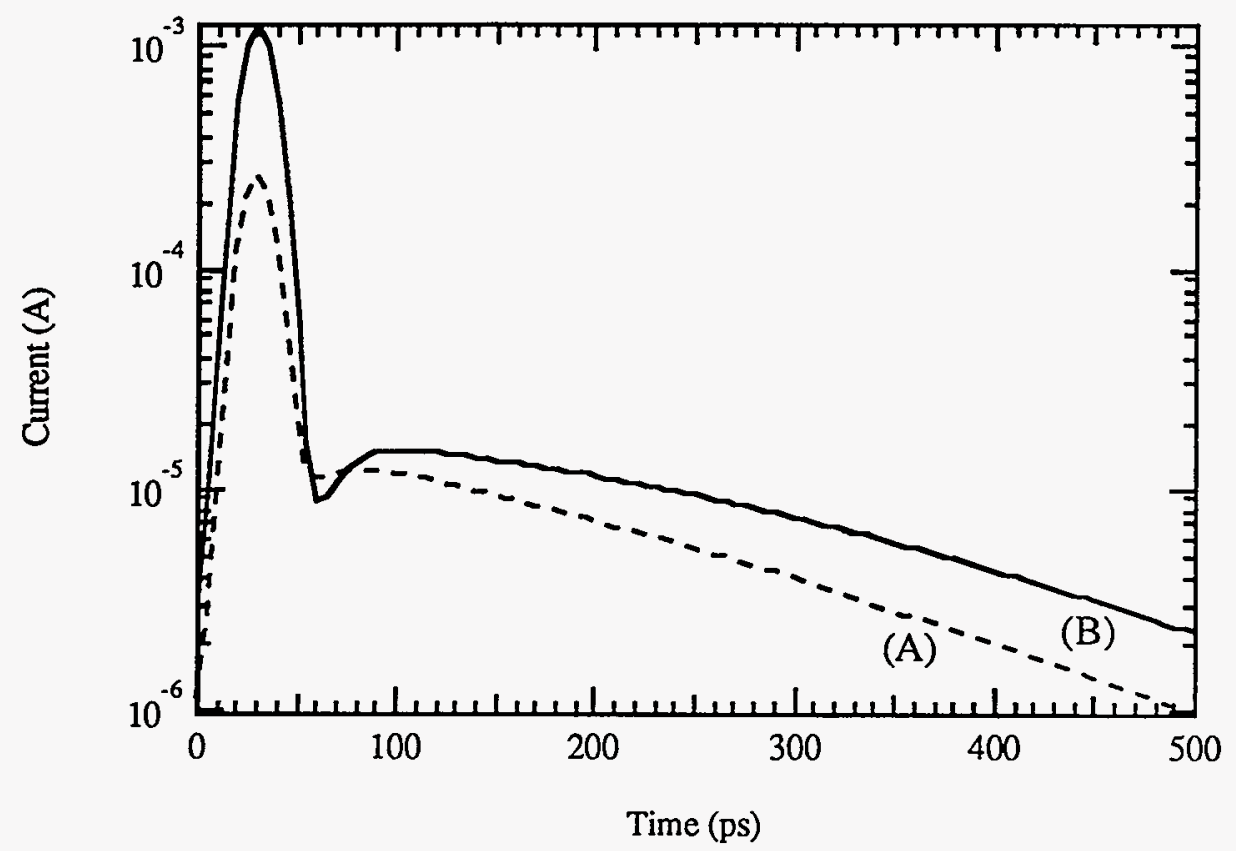

Figure 4.3.25: Calculated current transient for ions parallel and normal to applied electric field direction; (A) calculated transient with the ions normal to the applied field direction; (B) calculated transient with ions parallel to the applied field direction. The calculated transients are smoothed with a Gaussian response function of width of $18.6 \mathrm{ps}$. 
[1] A. E. Iverson, $\mathrm{PhD}$ Thesis, University of New Mexico, 1988

[2] R. S. Wagner, J. M. Bradley, N. Bordes, C. J. Maggiore, D N. Sinha and R. B. Hammond, IEEE Tans. Nucl. Sci., NS-34(6) (1987) 1240

[3] R. S. Wagner, N. Bordes, J. M. Bradley, C. J. Maggiore, A. R. Knudson, and A. B. Campbell, "Alpha-, boron, silicon- and iron- ion-induced current transients in low-capacitance silicon and GaAs diodes", preprint

[4] J. F. Ziegler, H. H. Andersen and J. Biersack, TRIM-90.5 Computer simulation code, Transport of ions in matter (1990)

[5] W. Seibt, K. E. Sundstrom and P. A. Tove, NIM, 113 (1973) 317

[6] E. C. Finch, NIM, 121 (1974) 431

[7] E. C. Finch and A. A. Cafolla, NIM, 198 (1982) 547

[8] R. M. Gilbert, G. K. Ovrebo, J. Schifano and T. R. Oldham, IEEE Trans. Nucl. Sci., NS-31(6) (1984) 1570

[9] R. M. Gilbert, G. K. Ovrebo and J. Schifano, IEEE Trans. Nucl. Sci., NS-32(6) (1985) 4098

[10] T. R. Oldham, F. B. McLean and J. M. Hartman, IEEE Trans. Nucl. Sci., NS33(6) (1986) 1646

[11] R. B. Adler, A. C. Smith, and R. L. Longini, Introduction to Semiconductor Physics, Semiconductor Electronics Education Committee, Vol. 1, Educational Services, Inc. (1964)

[12] A. Evan Iverson, and D. L. Smith, J. Appl. Phys., 61 (1987) 234

[13] A. Evan Iverson and D.L. Smith, Los Alamos Report LAUR-86-3986, 5/26/87 (submitted to IEEE Transactions on Electron Devices)

[14] D. L. Scharfetter and H. K. Gummel, IEEE Trans.. Elec. Dev., ED-16 (1969) 64 
[15] C. A. Hewett, J. R. Zeidler, M. J. Taylor, C. R. Zeisse, and K. L. Moazed, in New Diamond Science and Technology, Ed. by R. Messier, J. T. Glass, J. E. Butler and R. Roy, Pittsburgh, MRS, (1991) 1107

[16] V. Venkatesan and K. Das, IEEE Elec. Dev. Lett., 13(2) (1992) 126

[17] L. S. Pan, D. R. Kania, P. Pianetta and O. L. Landen, Appl. Phys. Lett., 57(6) (1990) 623

[18] P. A. Markowich, The Stationary Semiconductor Device Equations, Wien, New York, Springer-Verlag, 1986

[19] S. Kirkpatrick, IEEE Trans. Elec. Dev., ED-26(11) (1979) 1742

[20] R. M. Gilbert, G. K. Ovrebo and J. Schifano, IEEE Trans. Nucl. Sci., NS-32(6) (1985) 4098

[21] J. N. Bradford, IEEE Nucl. Sci., Correspondence, (1978) 1144

[22] A. Taroni and G. Zanarini, NIM, 67 (1969) 277

[23] W. Seibt, K. E. Sundstrom and P. A. Tove, NIM, 113 (1973) 317

[24] L. S. Pan, PhD thesis, Stanford University, 1991

[25] V. A. J. van Lint, Proc. IEEE, 62(9) (1974) 1190

[26] D. K. Nichols and V. A. J. van Lint, IEEE Trans. Nucl. Sci, NS-13(6) (1966) 119 


\section{Chapter 5: Conclusions}

A number of fairly clear conclusions can be drawn from the various measurements that have been discussed in this dissertation. For the first time, the noninjecting character of $\mathrm{Ti} / \mathrm{Au}$ of $\mathrm{Ti} / \mathrm{Pt}$ contact systems on diamond has been demonstrated. This contact characteristic can be considered to be a manifestation of a large surface recombination at the detector contacts. It can be speculated that the reaction of titanium with diamond to form TiC may result in a high density of surface trapping/recombination sites and thereby give rise to a large surface recombination velocity. This hypothesis is consistent with the observed linear I-V characteristics of the contacts [1].

All of the diamond detectors studied show charge collection characteristics consistent with a mean energy to produce an electron-hole pair of $14.1 \pm 0.5 \mathrm{eV}$. This is also consistent with the value of $13.25 \mathrm{eV}$ reported by Canali, et. al. [2]. Further, from measurements of the mean value of the charge collected when monoenergetic $\alpha$-particles interact with natural diamond, and the observation of significant low-energy tailing in the resulting pulse-height spectra, incomplete charge collection is severe, even with applied fields as high as $200 \mathrm{KV} / \mathrm{cm}$ and detector thicknesses as small as $14.5 \mu \mathrm{m}$.

Application of a reasonable model to the field-dependent charge collection data shows that there is preferential trapping of electrons notwithstanding the high level of nitrogen impurity known to be present in the type IIA natural diamond. Although the model suggests variations in the $\mu \tau$-products for carriers from specimen to specimen by as much as a factor of three, the preferential trapping of electrons is always found. The asymmetry observed in charge collection data with varying polarity of the electric field implies that the $\mu \tau$-product for holes is larger than for electrons in this type of diamond.

While natural diamond has and can continue to have applications as a radiation detector for charge particles, it is evident that the resolution available is severely limited by the high density of traps. Diamond will approach its potential only with a 
considerable reduction in the trapping density, i.e., with a significant increase in the $\mu \tau$ products of the carriers. Furthermore, in order to describe the detector resolution properly, field-dependence of the carrier lifetimes appears to be necessary.

Time-resolved current transients from $5 \mathrm{MeV}$ helium and $10 \mathrm{MeV}$ silicon ions have, for the first time, allowed observation of the dynamics of the charge collection process in diamond with a time resolution of about $18.6 \mathrm{ps}$. By comparison of the measured transients with a one-dimensional transport model it has been demonstrated that the collapse of the applied electric field from the buildup of a space charge field and the high density of carrier traps combine to severely limit charge collection except from the periphery of the track of a heavy charged particle. The rise times of the transients were found to be significantly larger than the response time of the recording system, and appear to be driven by the factors just discussed. Although predicted by the onedimensional model, no experimental evidence has been found for a large initial current transient from the earliest separation of holes and electrons. From considerations of the predicted fraction of collected charge associated with this feature, $\leq 10 \%$, and the slowing down time of ions in diamond, a recording system with response time of less than $1 \mathrm{ps}$ will be required for an attempt to determine if this feature actually exists.

The one-dimensional model may be reasonable in describing the response of the diamond detectors, at least qualitatively, to the ion excitations with low-excitation densities, but appears inadequate for heavy-ions with high-excitation densities.

Finally, charge collection seems to be greater by a factor of about 2.5 when the ion track is parallel to applied electric field as opposed to normal to the field. This result supports the prediction by Nichols, et. al. [3] and van Lint [4]. 
[1] E. H. Rhoderick and R. H. Williams, Metal-Semiconductor Contacts, Second Ed., Oxford University Press, Oxford (1988)

[2] C. Canali, E. Gatti, S. F. Kozlov, P. F. Manfredi, C. Mangredotti, F. Nava and A. Quirini, NIM, 160 (1979) 73

[3] D. K. Nichols and V. A. J. van Lint, IEEE Trans. Nucl. Sci, NS-13(6) (1966) 119

[4] V. A. J. van Lint, Proc. IEEE, 62(9) (1974) 1190 


\section{Chapter 6: Is there life after this thesis? (Future research directions)}

The experimental and modeling efforts presented in this dissertation have only scratched the surface of the study of detector characteristics of diamond. There is also no reason to believe that we cannot apply all of the models developed here to other semiconductor materials. We envision future research efforts to be focused on supporting and extending the type of results reported here, and to attempt to examine some of the speculations presented.

First of all, we need to obtain better diamond, natural or otherwise, to improve the quality of the charge collection experiments, especially in the critical low-field region below $10 \mathrm{KV} / \mathrm{cm}$. This should be done to further confirm the charge collection model presented in Sec. 3.2. Secondly more theoretical examinations, such as the variation of carrier lifetime as a function of applied electric field, are needed to increase the understanding of the detector resolution results presented in Chap. 3.

The large initial current due to dielectric collapse that is predicted by the onedimensional transport model was not observed experimentally. This can be attributed to the fact that the dielectric collapse may occur as the ions slow down in the detector thereby resulting in a much smaller initial current. Some efforts should be expended in trying to observe this initial effect with other systems.

As shown from the Si-ion excitation, modeling of the response of RIC diamond detectors to heavy ion excitations may require at least a 2-D treatment. Such development will further enable us to include more realistic models of the initial excess carrier distribution.

The experiments presented here were limited to natural diamond. As mentioned in Chap. 1, large area polycrystalline CVD diamond with carrier transport properties similar to natural diamond can now be made, and there is no theoretical reason why CVD 
diamond cannot be produced with electrical properties superior to that of natural diamonds. We believe that this is just matter of time. Therefore, we can use the methods shown in Chap. 3 to examine the properties of polycrystalline CVD diamond for heavy ion detection purposes.

Of course, the natural extension of the above application is to use it as a screening method to guide the growth process of CVD diamond to improve the electric quality of the material. The advantages of the methods are: $i)$ the measurement arrangement can be made to be extremely compact; ii) automatic data collection and analysis can be easily accomplished; and iii) the technique allows us to obtain estimates of mean transport properties, separately, and with considerable ease .

We would like to be able to produce injecting contacts for diamond. This might be achieved by ion-implantation methods. Given injecting contacts, there is no technical reason why a RIC detector with gain significantly greater than unity cannot be produced. Also, a detector structure similar to a $\mathrm{p}^{+}-\mathrm{i}-\mathrm{n}^{+}$diode can be envisioned where heavily doped regions on either side of the intrinsic region can be made. One would operate it with forward bias. Because diamond is an electrical insulator and thus leakage current should be much less than that of present detectors made from Si or GaAs under the same operating conditions. 


\section{Appendix A: \\ Photolithography and lift-off and wet/dry-etch processes: the recipes}


The material presented in this section represents the recipes utilized to fabricate the detectors used in the time-resolved experiments. The recipes start after detector preparation as described in Sec. 2.1.

It is important to emphasize that these are only recipes. There may be other procedures that may provide the same or similar results.

\section{RECIPE FOR LIFT-OFF PROCESS:}

1. Mount a diamond sample onto a 3" silicon wafer with small amount of photoresist. The silicon wafer is handled through out the fabrication procedure.

2. Bake the sample at $90^{\circ} \mathrm{C}$ on a hot plate for approximately one minute.

3. Set the speed and time on the photoresist spinner to $6000 \mathrm{rpm}$ and 30 sec., respectively.

4. Pour enough photoresist onto the sample to cover it completely.

5. Spin the photoresist.

6. Bake the sample at $90^{\circ} \mathrm{C}$ on a hot plate for 5 minutes.

7. Repeat steps 4 and 5.

8. Bake the sample at $90^{\circ} \mathrm{C}$ in a convection oven for 15 minutes.

9. Submerge in trichloroaethylene for 10 minutes. (This step hardens the photoresist to produce "ledges" at the edges of the photoresist walls from photolithograhic patterning.)

10. Expose the photoresist for an appropriate length of time. Determine the required exposure time by performing several test exposures. In our process, we used Kasper contact aligner. For this aligner, the exposure time of $19 \mathrm{sec}$. was used.

11. Develop the photoresist. 
12. Load the sample into a metal deposition system (evaporation or sputtering apparatus). It is important to remember that the sample temperature must be kept below $120^{\circ} \mathrm{C}$. Beyond this temperature, the photoresist will begin to "burn".

13. After metal deposition, submerge the sample in an acetone bath for several minutes. Unwanted metall will begin to lift from the sample. Leave the sample in the bath long enough for the unwanted metal to lift-off completely. If some unwanted metal stays intact, use an ultrasonic cleaner to assist in the lifting of the metal.

14. Rinse the sample with de-ionized water and blow dry with a $\mathrm{N}_{2}$ gun.

\section{RECIPE FOR WET/DRY ETCH PROCESS:}

1. Load the sample into a deposition system and deposit desired metals. (Sample temperature is irrelevant since there is no photoresist on the sample.)

2. Follow steps 1 through 8 from the lift-off process.

3. Using the aligner, pattern the device on the sample. In this case, the pattern on the photo mask must be the negative image of the photo mask used in the lift-off process.

4. Bake the patterned sample at $120^{\circ} \mathrm{C}$ in a convection oven for approximately 30 minutes.

5. Either dry etch the unwanted metal with a reactive-ion plasma or wet etch with chemical solutions such as $\mathrm{KI}$ or acids.

6. After etching process, remove the photoresist with acetone.

7. Rinse the sample with de-ionized water and blow-dry with $\mathrm{N}_{2}$ gun. 
Etchants commonly used to fabricate diamond detectors during the wet-etch process:

Titanium: $\quad \mathrm{HF}: \mathrm{HNO}_{3}: \mathrm{H}_{2} \mathrm{O}$

Platinum: $\quad \mathrm{Hot}\left(90^{\circ} \mathrm{C}\right) \mathrm{HCl}: \mathrm{HNO}_{3}$

Gold: $\quad \mathrm{KBr}$ or $\mathrm{KI}$ solution

Aluminum: $\quad \mathrm{H}_{3} \mathrm{PO}_{4}: \mathrm{HC}_{2} \mathrm{H}_{3} \mathrm{O}_{2}$

Silver:

$\mathrm{HNO}_{3}: \mathrm{HF}$

$(1: 1)$ 


\section{Appendix B: \\ Experimental arrangement used to perform transient photoconductivity measurements}


The transient photoconductivity measurements can be considered to be classical conductivity modulation measurements. As the name implies, the excitation or the conductivity modulation of a material is done via photons, usually from a laser. One of the advantages of using lasers is that by adjusting either the energy of the laser or by using neutral density filters (these are usually filters to limit the transmission of the laser intensity) the free carrier density can be controlled with an enormous range; typically the range is over 4 orders of magnitude.

The experimental arrangement (Fig. B.1) is relatively simple. The measurements are typically performed by intrinsically exciting diamond (this entails exciting electrons from the valence band up to the conduction, directly). To do this, a laser with the photon energy, in the ultraviolet spectral range, greater than $5.5 \mathrm{eV}$ is necessary. In order to simplify the ensuing data analysis, the excitation pulse width is made sufficiently short as compared to the free carrier lifetime to achieve impulse excitation. The first step in generating ultraviolet laser at the required photon energy is generating $2.04 \mathrm{eV}$ light from a dye laser that is sync-pumped by a mode-locked Nd:YAG [1] with the repetition rate of $2.5 \mathrm{~Hz}$ or $10 \mathrm{~Hz}$. Non-linear mixing crystals of potassium dihydrogen phosphate (KDP) and $\mathrm{B}-\mathrm{BaBO}_{4}$ are used to frequency triple the laser from the dye laser. This tripling process results in a ultraviolet laser with the photon energy of $6.11 \mathrm{eV}$. The pulse length of this laser is $2-5$ ps with the energy of the pulse at $50 \mu \mathrm{J}$, typically. A nominal lifetime of mean carriers in diamond is typically 200 ps up to as large as few nanoseconds. This means that the laser pulse length of 2-5 ps can be considered to be instantaneous and thus satisfies the impulse excitation requirement.

As mentioned in Chap. 2, diamond detectors are incorporated into 50- $\Omega$ striplines with a gap in the center of the diamond. One end of the detector is connected to an micro-ammeter, which measures the leakage current, with the meter being connected to a bias supply. The other end of the stripline is routed to a Tektronix $7250,6-\mathrm{GHz}$, singleshot oscilloscope which records the current transient induced from the ultraviolet laser 
impulse excitation. The rise time of the recording system is about $60 \mathrm{ps}$. The recorded current waveform is then transferred to a computer for analysis of the experimental data.

The principle information gained from this type of measurements is the mean carrier mobility and lifetime. This is possible since the detector output current has temporal information, i.e., shape of the current output pulse determines the mean carrier lifetime, and the integral of the current pulse results in the mean carrier $\mu-\tau$ product. Because the mean carrier lifetime is obtained from the current pulse shape, the mobility is derived by dividing the $\mu-\tau$ product by the lifetime. This type of analysis was done to derive the laser data shown in Chap. 3
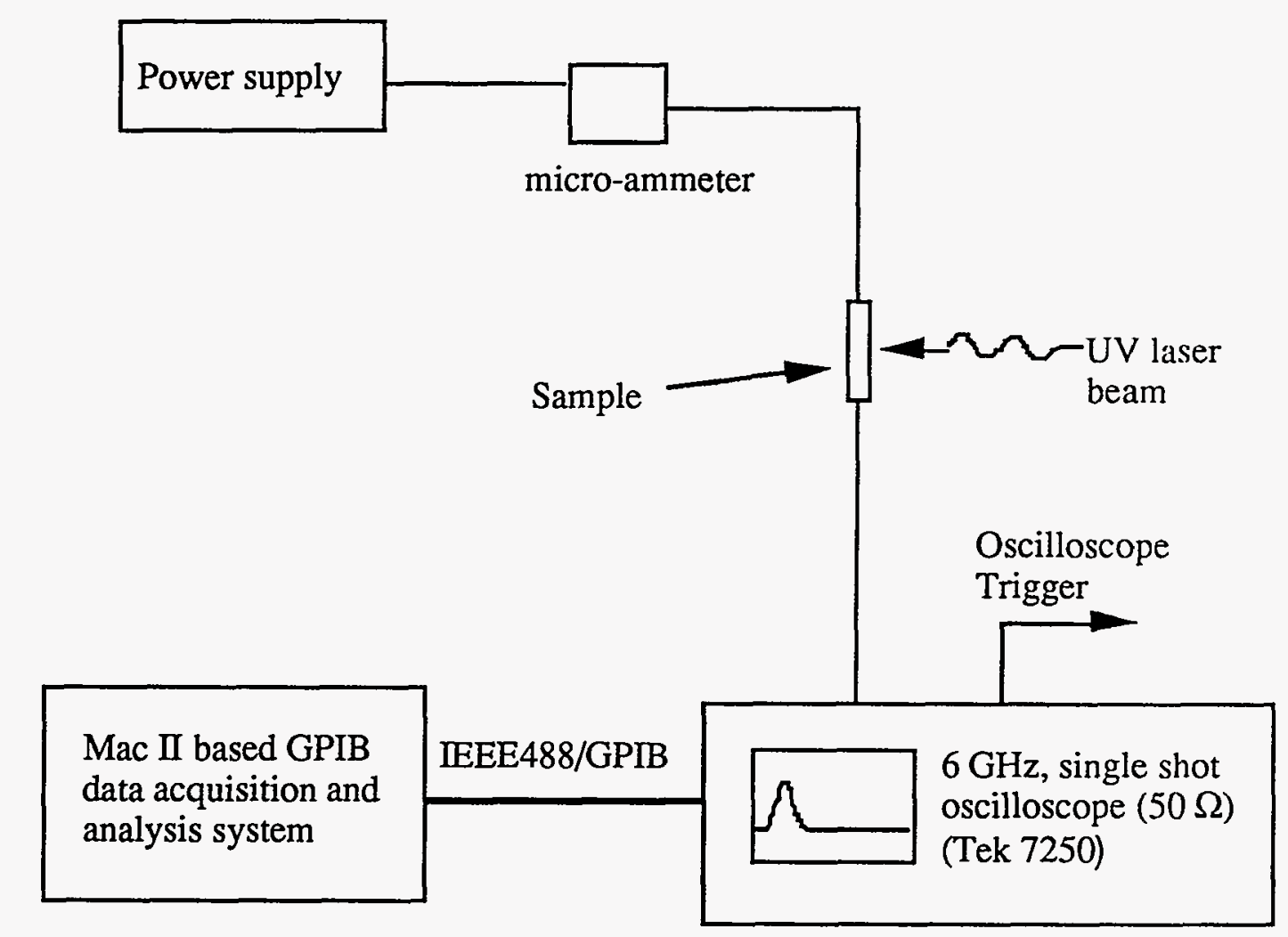

Figure B.1: Schematic of the arrangement used to measure transient photoconductivity of diamond detectors. 


\section{References}

[1] O. L. Landen, M. D. Perry and E. M. Campbell, Phys. Rev. Lett., 60 (1988) 1270 


\section{Appendix C:}

$O^{\text {th }}$ order calculation of the induced charge in a radiation-induced conductor under 1) $\infty$-carrier recombination at the boundaries and 2) carrier injection at the boundaries 
In the cases examined here the view of the model photoconductor is featured in Fig. C.1.

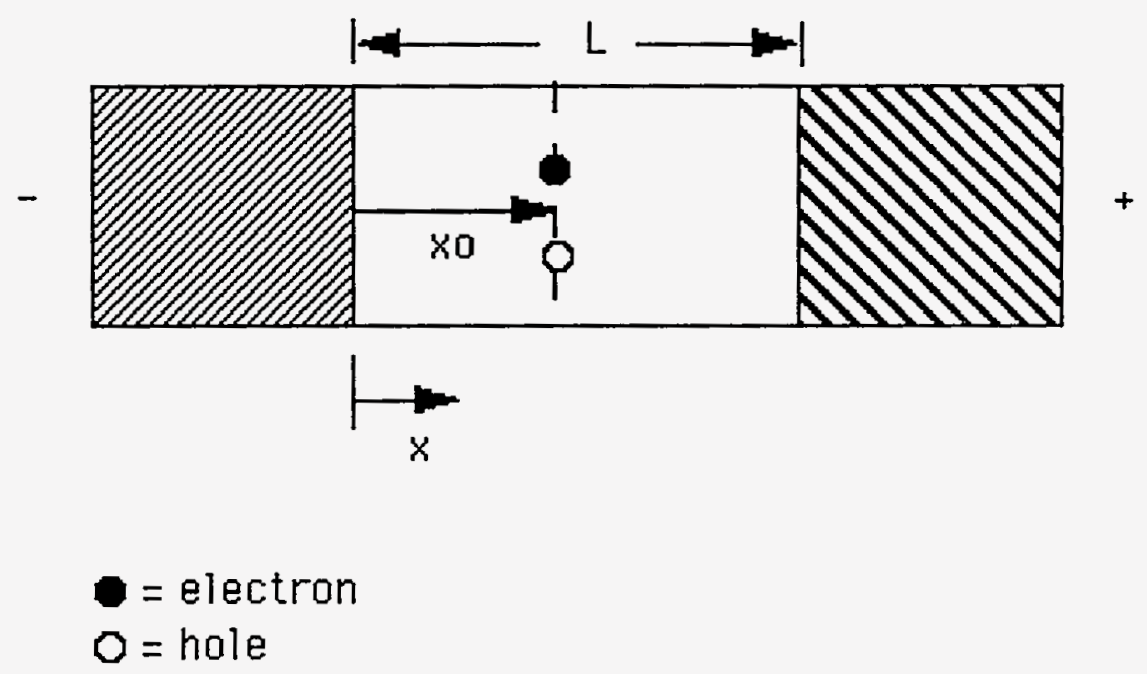

Figure C.1: Schematic of the photoconductor being modeled.

It is assumed that electrons and holes diffuse freely without coupling in their motion.

Case 1: $\infty$-recombination at the boundaries

In this case, the photoconductor is not necessarily space charge neutral. With this in mind a good starting point is Ramo's theorem. This theorem states that

$$
\frac{d q}{Q}=\frac{d x}{L}
$$

where $Q=$ free charge in the device

$\mathrm{dq}=$ the infinitesimal charge induced by the $\mathrm{Q}$ moving $\mathrm{dx}$

Consider electrons first. Since there is no charge injection at the contacts, the created charge has an exponential distribution in time

$$
Q_{e}=Q_{o} \exp \left(-\frac{t}{\tau_{e}}\right)
$$




$$
\begin{aligned}
\text { where } \mathrm{Q}_{0} & =\text { the magnitude of the charge attributed to free electrons } \\
\tau_{\mathrm{e}} & =\text { free electron life time }
\end{aligned}
$$

Using equations $\mathrm{C}_{\mathrm{i}} 1$ and $\mathrm{C} .2$ for the electrons

$$
d e=\frac{Q_{o}}{L} \exp \left(-\frac{t}{\tau_{e}}\right) d x
$$

but $\mathrm{t}=\mathrm{x} / \mathrm{v}$, where $\mathrm{v}=$ drift velocity

$$
d e=\frac{Q_{o}}{L} \exp \left(-\frac{x}{v_{e} \tau_{e}}\right) d x
$$

Assuming the drift velocity is independent of position and constant, an integration, with the limits from $x_{0}$ to $L$ is performed to get

$$
\Delta e=\frac{Q_{o}}{L} \nu_{e} \tau_{e}\left(1-\exp \left(-\frac{\left(L-x_{o}\right)}{v_{e} \tau_{e}}\right)\right)
$$

Now, following the same logic for the holes, we obtain

$$
\Delta h=\frac{Q_{o}}{L} v_{h} \tau_{h}\left(1-\exp \left(-\frac{x_{o}}{v_{h} \tau_{h}}\right)\right)
$$

The total charge induced at the contacts is given by the summation of both electron and hole contributions

$$
\Delta q=\Delta e+\Delta h
$$

Substituting equations C.5 and C.6 into equation C.7, the following results

$$
\Delta q=\frac{Q_{o}}{L} v_{e} \tau_{e}\left(1-\exp \left(-\frac{\left(L-x_{o}\right)}{v_{e} \tau_{e}}\right)\right)+\frac{Q_{o}}{L} v_{h} \tau_{h}\left(1-\exp \left(-\frac{x_{o}}{v_{h} \tau_{h}}\right)\right)
$$

As an idealization, assume $v_{e} \tau_{e}=v_{e} \tau_{e}=v \tau$. Then, equation C. 8 reduces to 


$$
\Delta q=\frac{Q_{o}}{L} \nu \tau\left(\left(1-\exp \left(-\frac{\left(L-x_{o}\right)}{\nu \tau}\right)\right)+\left(1-\exp \left(-\frac{x_{o}}{\nu \tau}\right)\right)\right)
$$

From this expression, we can see that the total charge induced is a function of space. Also, under this assumption, the ultimate lifetime of a carrier is the transit time of the carrier.

Equation C. 9 can be expressed in terms of the three dimensionless variables

$$
\begin{aligned}
& q^{\prime}=\frac{\Delta q}{Q_{o}} \\
& \beta=\frac{v \tau}{L} \\
& x^{\prime}=\frac{x_{0}}{L}
\end{aligned}
$$

The resulting expression is

$$
q^{\prime}=\beta\left(2-\exp \left(-\frac{\left(1-x^{\prime}\right)}{\beta}\right)-\exp \left(-\frac{x^{\prime}}{\beta}\right)\right)
$$

The distribution of $\mathrm{q}^{\prime}$ as a function of $\beta$ and the coordinate of the charge creation, $\mathrm{x}^{\prime}$, is shown in Fig. C.2. 


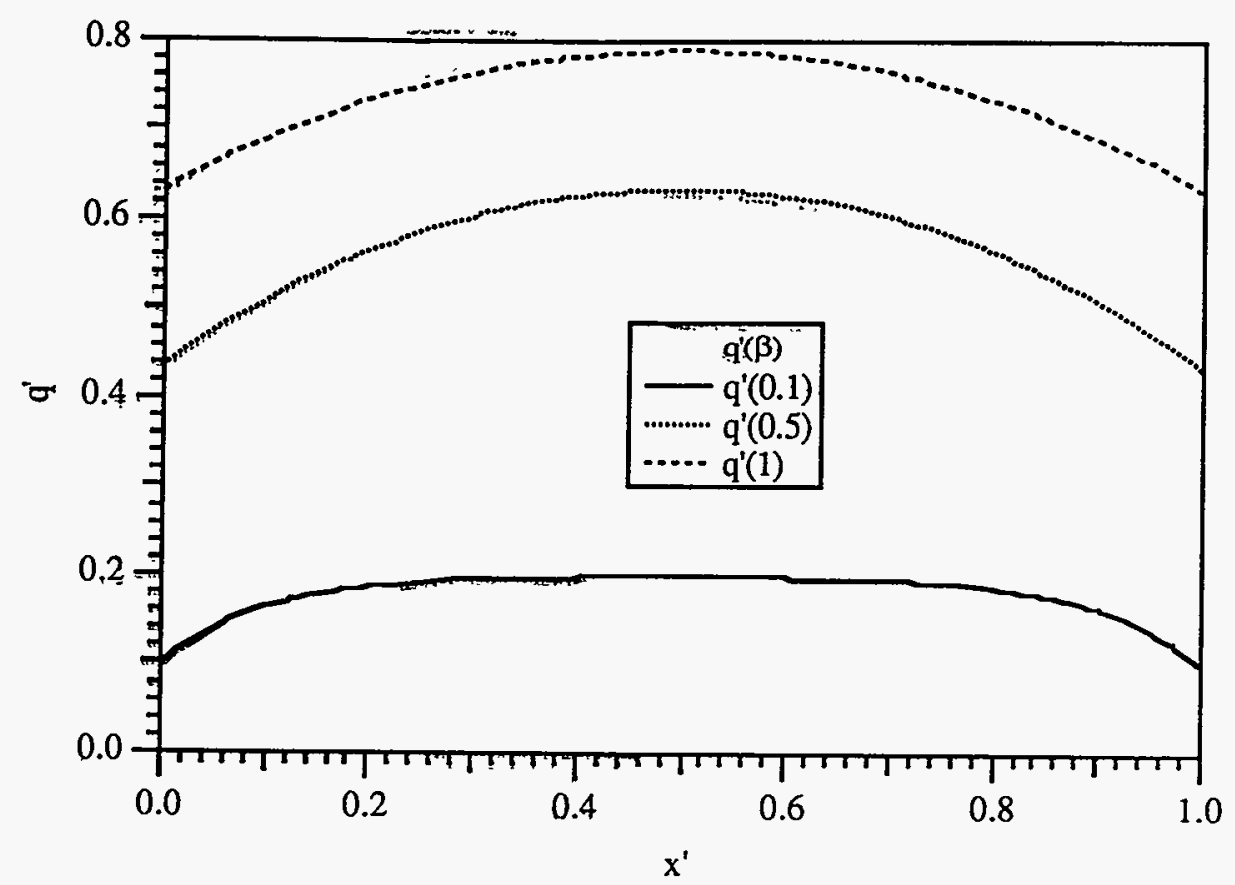

Figure C.2: Distribution of the total induced charge as a function of initial electronhole pair position and the ratio of çarrier mean free path.

The individual contributions are shown in Fig. C.3. It is important to notice that full charge collection cannot occur in this case due to the exponential distribution for the carrier lifetime . This is also shown in equation C.10. 


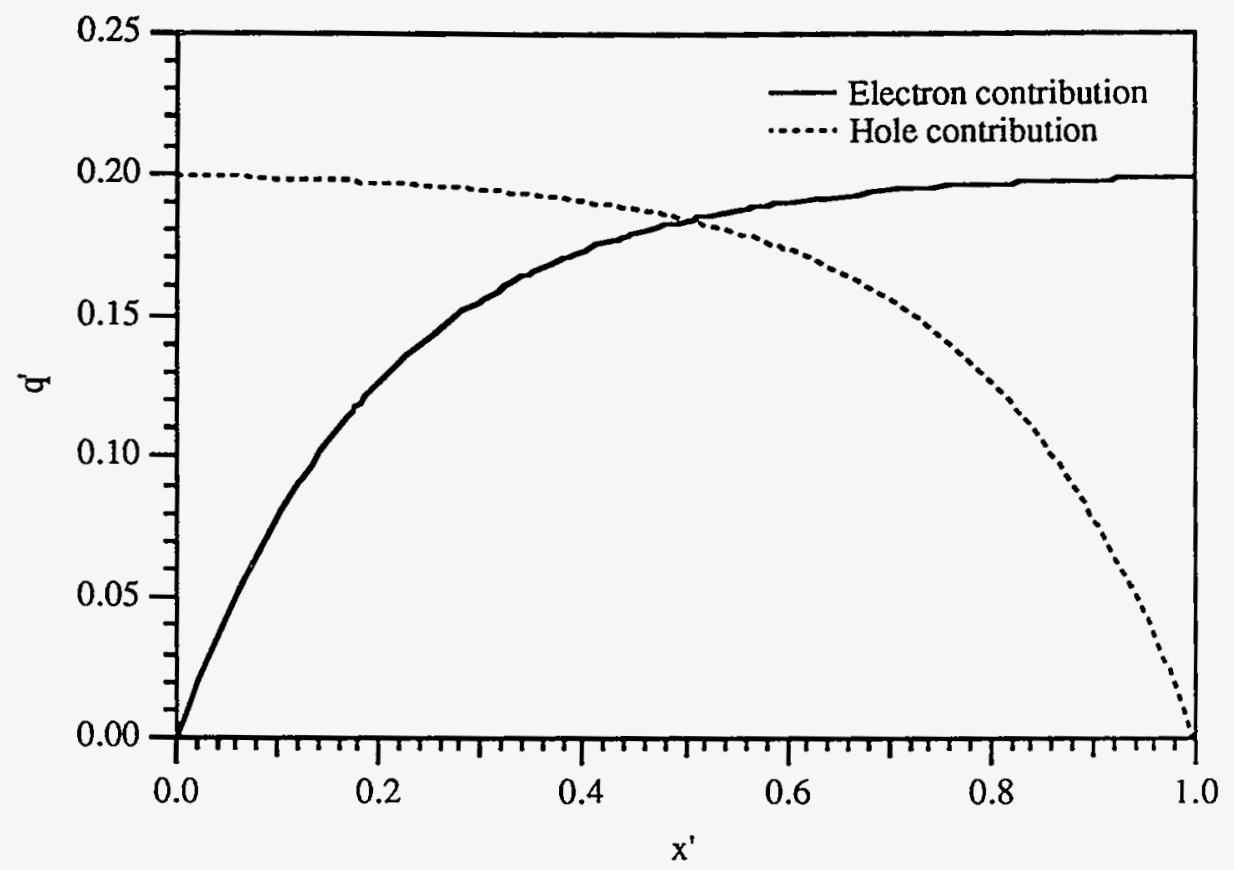

Figure C.3: The spatial distribution of induced charge by individual type of carrier at $\beta=1$.

Case 2: carrier injection of the same type at the opposite boundary

This case is much simpler than the former. Again, Ramo's theorem is utilized. Also, at any time of the device operation, the space charge neutrality is maintained and the applied field is uniform during the motion of the charge carriers.

In this case, the induced charge comes strictly from the motion of the carriers. Carriers are injected into the device as the carriers leave the device to maintain the space charge neutrality. Under these conditions the integration limits of the Ramo's theorem go from 0 to $\lambda$, the mean free path $(=v \tau)$

$$
\Delta(\text { e or } h)=Q_{o} \int_{0}^{\lambda(e \text { or } h)} \frac{d x}{L}
$$


Thus, the total induced charge is

$$
\Delta q=Q_{o}\left(\frac{v_{h} \tau_{h}+\nu_{e} \tau_{e}}{L}\right)
$$

We can arrive at the same expression as in $\mathrm{C} .12$ by using the exponential distribution

$$
p(t)=e^{-(t / \tau)}
$$

for the probability for the carrier survival. With the boundary condition associated with this case the limits of integration are 0 to $\infty$

$$
\Delta(e \text { or } h)=Q_{o} \int_{0}^{\infty} p(t) \frac{v}{L} d t
$$

Performing the integration, the result is the same as C.12.

For the ideal case of $v_{e} \tau_{e}=v_{e} \tau_{e}=v \tau$ and in dimensionless variables, the following expression results

$$
q^{\prime}=2 \beta
$$

In this expression, the spatial dependence disappears. The only variable of any importance is the mean free path. 


\section{Appendix D:}

$5 \mathrm{MeV}$ alpha-particle damage of single crystal, natural type IIa and polycrystalline CVD diamonds 


\section{Introduction}

In order to achieve the objectives planned for the Superconducting Supercollider (SSC), it is essential that the system's detectors withstand the extreme radiation fields from the high-energy and high-intensity beams anticipated during its operation. In all likelihood, silicon will be chosen as the semiconductor material for fabrication of solidstate detectors. One major drawback to silicon is its susceptibility to radiation damage [1].

One possible alternative to silicon is diamond $[2,3]$. This material has become viable due to considerable improvement in the quality of polycrystalline, chemical vapor deposited (CVD) diamond films [4]. These have the potential for providing low-cost, high-purity material for development of fast, large-area, and radiation-hard detectors.

By virtue of bond strengths alone, diamond should be much more resistant to radiation damage than silicon. The average atomic displacement threshold energy of single-crystal, natural type IIa diamond has been measured as $43 \mathrm{eV}$ [5], about a factor of 2 greater than silicon. Therefore, under most radiation environments, diamond should be more resistant to damage than silicon.

Although some radiation damage work has been done on natural diamonds $[6,7]$, very little data has been reported on radiation damage effects to electrical properties of CVD diamond. In this paper we present studies of radiation damage effects on CVD films. We have utilized beams of $5 \mathrm{MeV} \mathrm{He}+$ ions to produce damage, Raman and photoluminescence (PL) spectroscopies to examine structural changes, and transient photoconductivity (TPC) to measure the changes in electrical properties induced by the ion bombardment. For the natural diamond, we have been able to image the damaged area using confocal fluorescence microscopy. 


\section{Experimental}

\section{Diamond film preparation}

Polycrystalline CVD diamond film grown by microwave-assisted chemical vapor deposition on silicon using a methane and hydrogen gas mixture with nominal deposition conditions [8] was obtained commercially [9]. A film grown in this manner

has been shown to contain a nominal paramagnetic nitrogen concentration of $10^{17} \mathrm{~cm}^{-3}$ [10]. After deposition, the Si-substrate was chemically removed, yielding a freestanding, continuous diamond film with thickness of $120 \mu \mathrm{m}$. This was cut to produce samples of $0.5 \mathrm{~cm} \times 1.0 \mathrm{~cm}$ in size. For comparison purposes natural material/singlecrystal type IIa diamonds ( $35 \mu \mathrm{m}$ thick), supplied by DubbleDee Harris Corp., were used.

\section{Damage and measurement apparatus}

The diamond films were irradiated at room temperature with an ion beam of 5 $\mathrm{MeV} \mathrm{He}^{+}$ions (beam current of about $15 \mathrm{nA}$ ) (Fig. D.1). With the sample holder used, we were able to mask one half of each CVD film in order to compare damaged and undamaged regions on the same sample. This was not possible with natural diamonds because of the small size of the samples except the sample used to obtain data for Fig. D.2. Also, the sample used to obtain Fig. D.2 was mounted on an alumina substrate. Rutherford backscattering spectroscopy was used to check whether or not the beam was on the sample and the fluence to a specimen was monitored by integrating the incident beam current.

Raman and PL spectra were excited with the $488 \mathrm{~nm}$ line of an $\mathrm{Ar}^{+}$laser $(\approx 10$ $\mathrm{mW}$ ), with the ability to perform both single-point and spatially-resolved measurements $[11,12]$. For single-point measurements, the laser was focused to a spot size of $6 \mu \mathrm{m}$. For spatially-resolved measurements, the laser was focused with a cylindrical lens to form an illuminated line. The imaging detector at the end of the spectrometer was used to 
acquire up to 1024 spectra simultaneously with each spectrum corresponding to a unique point along the illuminated line.

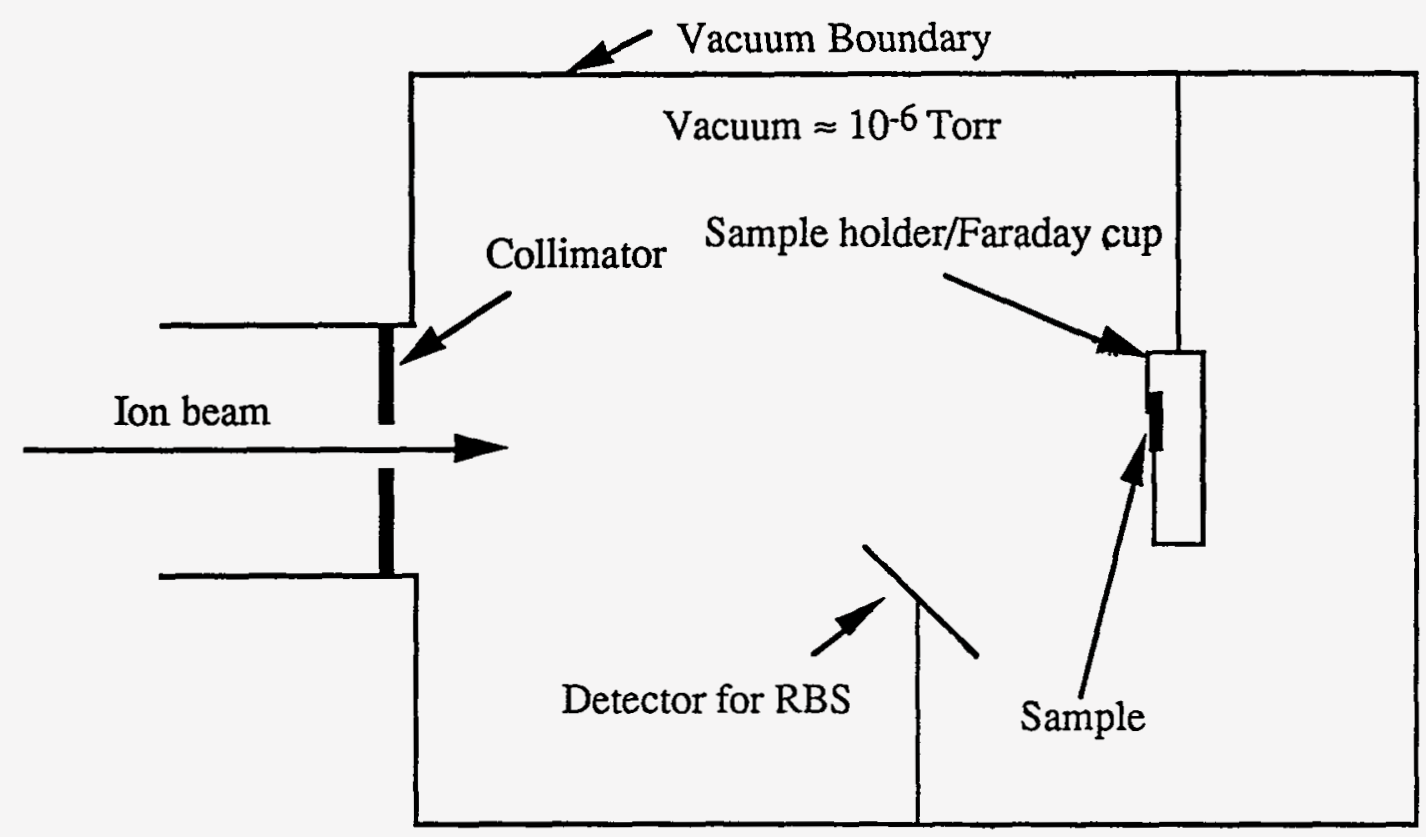

Figure D.1: Schematic of the apparatus used for damaging diamond films.

Electrical properties of the damaged and undamaged films were examined by using TPC (See App. B). The films were intrinsically excited with a 2-ps, $6.11 \mathrm{eV}$ laser beam, which creates a known quantity of free electron-hole pairs leading to changes in electrical conductivity. These changes were then measured with a $50-\Omega, 6 \mathrm{GHz}$, singleshot oscilloscope to permit measurement of carrier lifetimes and mobilities. It is important to note that the depth probed by the UV light is about 1-2 $\mu \mathrm{m}$, which is approximately 0.1 to 0.2 that of the range of the $5 \mathrm{MeV} \alpha$-particles, in diamond.

The damaged region of the natural diamond was imaged with Confocal microscopy [13] by exciting the region with the same excitation source as Raman and PL spectroscopies. The laser was optically filtered so that only the yellow luminescent line of $\mathrm{H} 3$ defect can be imaged. The images ware created by rastering the laser over the $\mathrm{x}-\mathrm{y}$ 
plane (surface) and by moving the sample in the $z$-direction with respect to the fixed focused laser. The resolution of the total imaging system is thought to be approximately $0.1 \mu \mathrm{m}$. In the future, this technique will be used in an attempt to image the damaged regions of polycrystalline CVD diamonds.

\section{Results and discussion}

All unirradiated natural diamonds used in this work exhibit Raman peaks centered at the wavenumber of $1332 \mathrm{~cm}^{-1}$ with widths in the range 2.1 to $2.8 \mathrm{~cm}^{-1}$ (Fig. D.2-ii). A small H3 PL peak (zero-phonon line, $2.461 \mathrm{eV}$ ), which is known to be characteristic of nitrogen-vacancy complex [7], is present in some of these samples. Unirradiated CVD

films also show the same Raman peak but with a width of $4.2 \mathrm{~cm}^{-1}$. They do not show the H3 PL peak.

Fig. D.2-i shows Raman and PL spectra from a damaged region in natural diamond irradiated to a total fluence of $8 \times 10^{15} \mathrm{~cm}^{-2}$. The H3 PL peak dominates the spectrum and the characteristic diamond Raman peak (absolute position of $19138 \mathrm{~cm}^{-1}$ ) is no longer seen due to the overwhelming strength of the PL peak. By enlarging the spectral region near $2.4 \mathrm{eV}$, other peaks (Table D.1) were observed, which, with the exception of the peak at $2.432 \mathrm{eV}$, can be assigned to phonon replicas.

$\mathrm{H} 3$ peaks are prominent in damaged and undamaged regions along with the characteristic diamond Raman peak at $1332 \mathrm{~cm}^{-1}$ (Fig. D.2-ii). Even at distances up to approximately $1.5 \mathrm{~mm}$ from the damaged region, the intensity of the H3 PL peak is far larger than the intensity of the H3 PL peak from an unirradiated sample. Adel, et al. [14], have shown that light-ions can induce annealing of the structural damage caused by irradiation. Thus, a possibility seems to exist for interstitials becoming mobile during light-ion irradiation, such as protons or $\alpha$-particles, and to react with vacancies to form $\mathrm{H} 3$ 

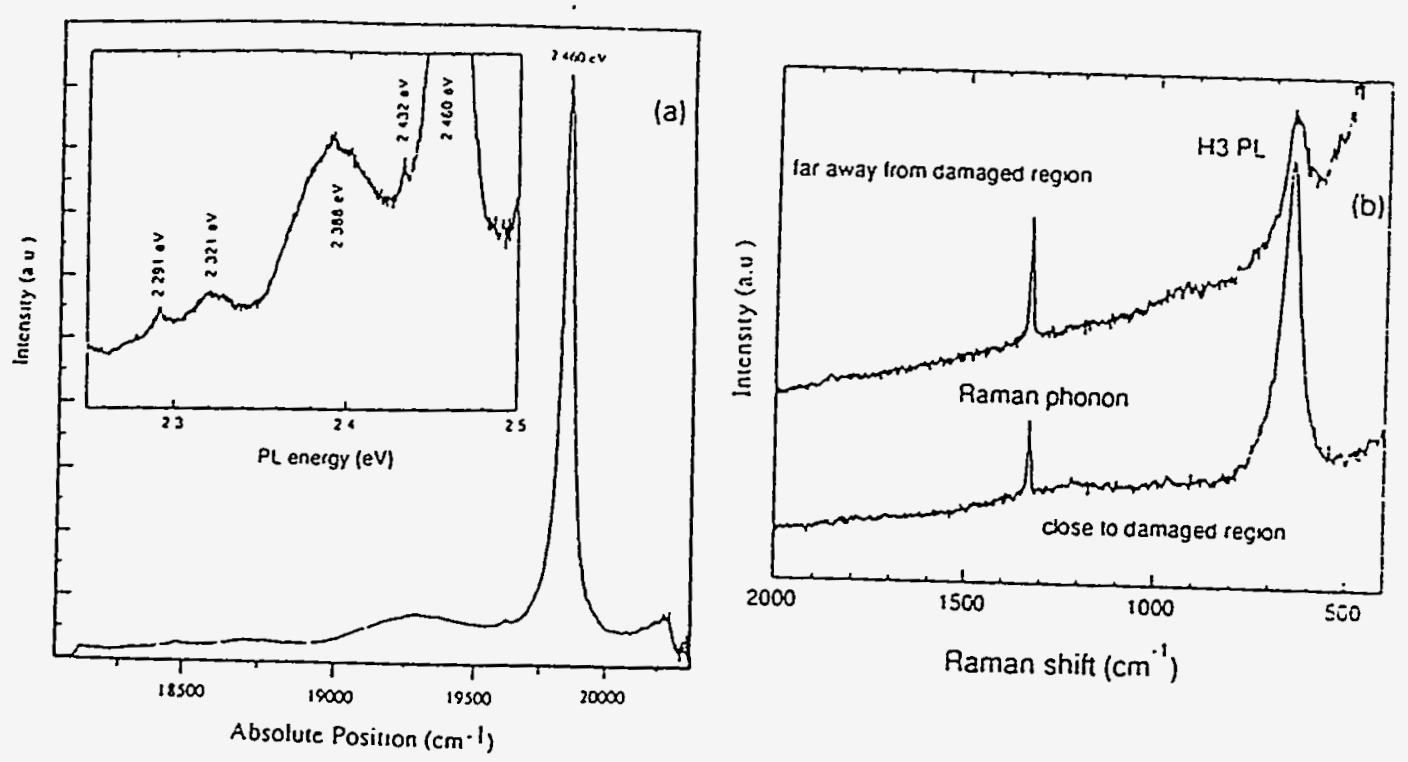

Figure D.2: Raman/PL spectra as a function of position; a) PL spectrum of the damaged region in $2 \mathrm{~mm} \times 2 \mathrm{~mm}$ single-crystal natural diamond irradiated to fluence of $8 \times 10^{15} \mathrm{~cm}^{-2}$ with the inset showing the magnified spectrum of the spectral region around the H3 $(2.460$ eV) PL peak; b) Raman/PL spectra from the regions close and far away from the damaged region. The ion beam collimation is done with a $0.5 \mathrm{~mm}$ diameter circular aperture with the sample mounted on an alumina substrate. The PL spectrum in i) is similar to the PL spectrum obtained by Perry et al. [15]. 
Table D.1: PL peak assignments for the peaks shown in the inset of Fig. D.2-i.

\begin{tabular}{|cc|}
\hline PL peak (eV) & Assignment \\
\hline 2.460 & $\mathrm{H} 3$ (zero phonon line, $2.461 \mathrm{eV})$ \\
2.432 & $? ? ?$ \\
2.388 & $\mathrm{H} 3+\mathrm{TA}\left(\mathrm{L}_{3}\right)$ \\
2.321 & $\mathrm{H} 3+2 \mathrm{TA}\left(\mathrm{L}_{3}\right)$ \\
2.291 & $\mathrm{H} 3+\mathrm{TA}\left(\mathrm{X}_{3}\right)+\mathrm{TA}\left(\mathrm{L}_{3}\right)$ \\
\hline
\end{tabular}

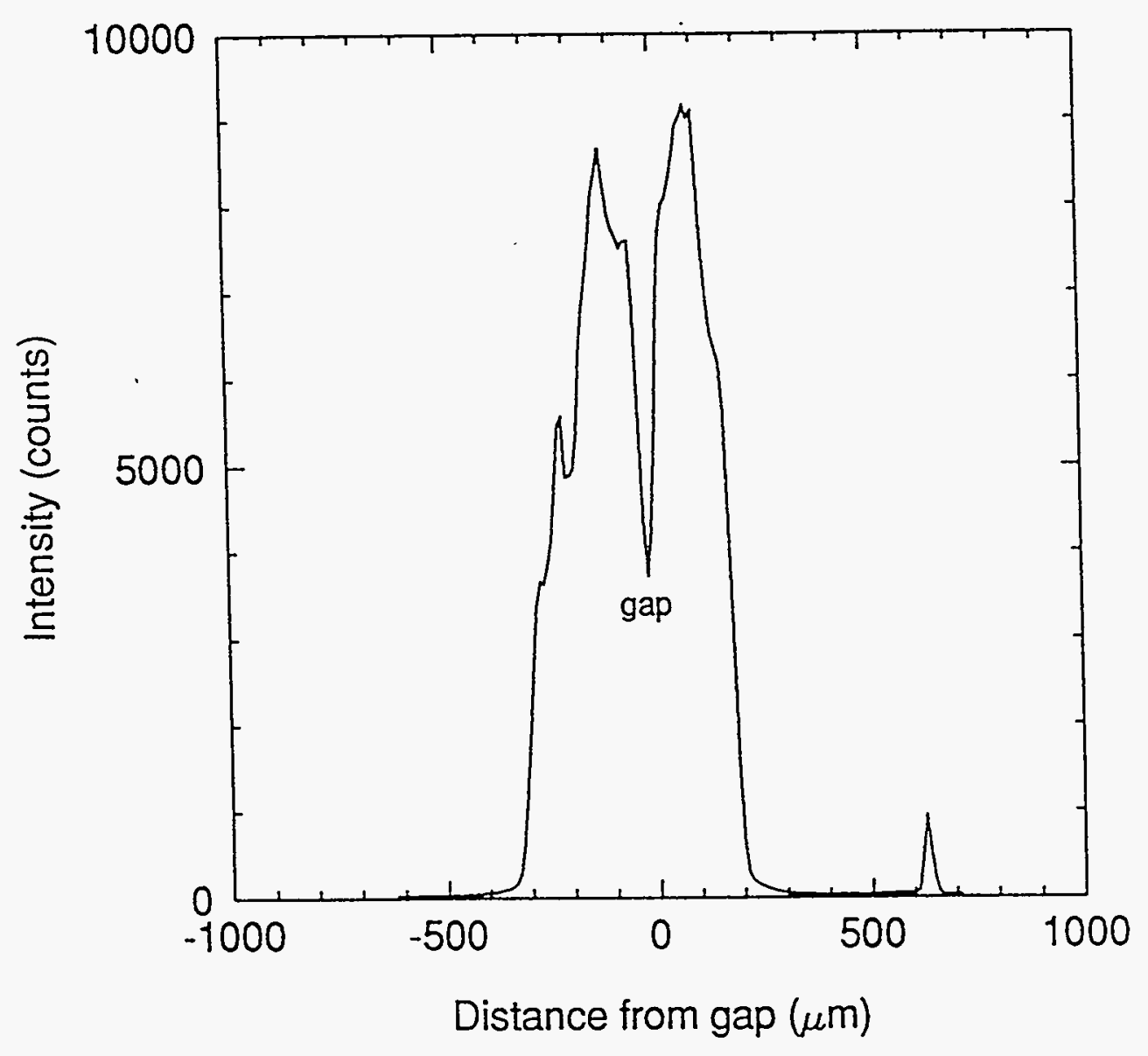

Figure D.3: Spatially-resolved H3 PL peaks across the damaged region of the natural diamond. The fluence on the sample is $8 \times 10^{15} \mathrm{~cm}^{-2}$. 0 $\mu \mathrm{m}$ denotes approximately the middle of the gap. 
defects. Based on irradiation conditions, fandom walk and absolute-rate theories [15], this possibility appears to be feasible.

Fig. D.3 shows spatially-resolved H3 PL peaks across the damaged region of the natural diamond. The depression of the intensity at approximately $0 \mu \mathrm{m}$ is a remnant from the imaging system. It is clear that the width of the peak distribution corresponds well with the diameter of the collimator, which was $0.5 \mathrm{~mm}$. Furthermore, there exists tails in the distribution. These are most likely due to the migration of the vacancies, as was shown in Fig. D.2-ii, from the damaged region. It is not clear, however, the origin of the satellite peak in the distribution at about $+570 \mu \mathrm{m}$. This is most likely due to the existing defects (such as inclusions) with the emissions close to $\mathrm{H} 3$ defect.

Fig. D.4 shows micrographs from confocal fluorescence microscopy of the damaged region in the natural diamond. Fig. D.4-a shows the surface image of the damaged region in the center of the sample. The size of the image matches well with the collimator size and it also matches the width of the $\mathrm{H} 3$ peak distribution shown in Fig. D.3. It is clear that there is another luminescent area with the emissions at the same energy as the $\mathrm{H} 3$ defect in the upper left hand corner of the micrograph. The distance from the center of damaged region to the center of the luminescent region is approximately $570 \mu \mathrm{m}$. This corresponds at to the satellite peak shown in Fig. D.3. Fig. D.4-b shows the depth image of the damaged area with both calculate ionization and vacancy distributions from TRIM-90.5 overlaid on the image. The peak of the vacancy distribution appears to correspond reasonably well with the maximum intensity in the image. But we can also see that the bright area of the image is very broad. This is most likely due to the vacancy migration in the sample during ion irradiation.

In Fig. D.5, Raman spectra of both irradiated and unirradiated regions of the CVD samples subjected to fluences of $1.6 \times 10^{12}$ and $1.6 \times 10^{14} \mathrm{~cm}^{-2}$ are shown. For 
a)

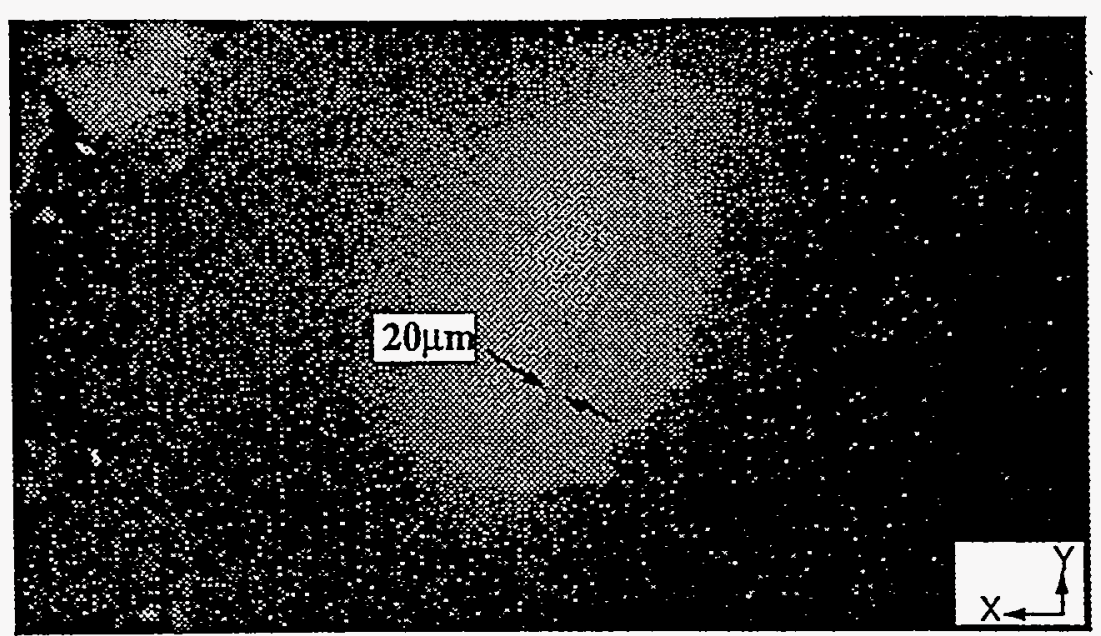

Laser beam direction for confocal imaging

b)

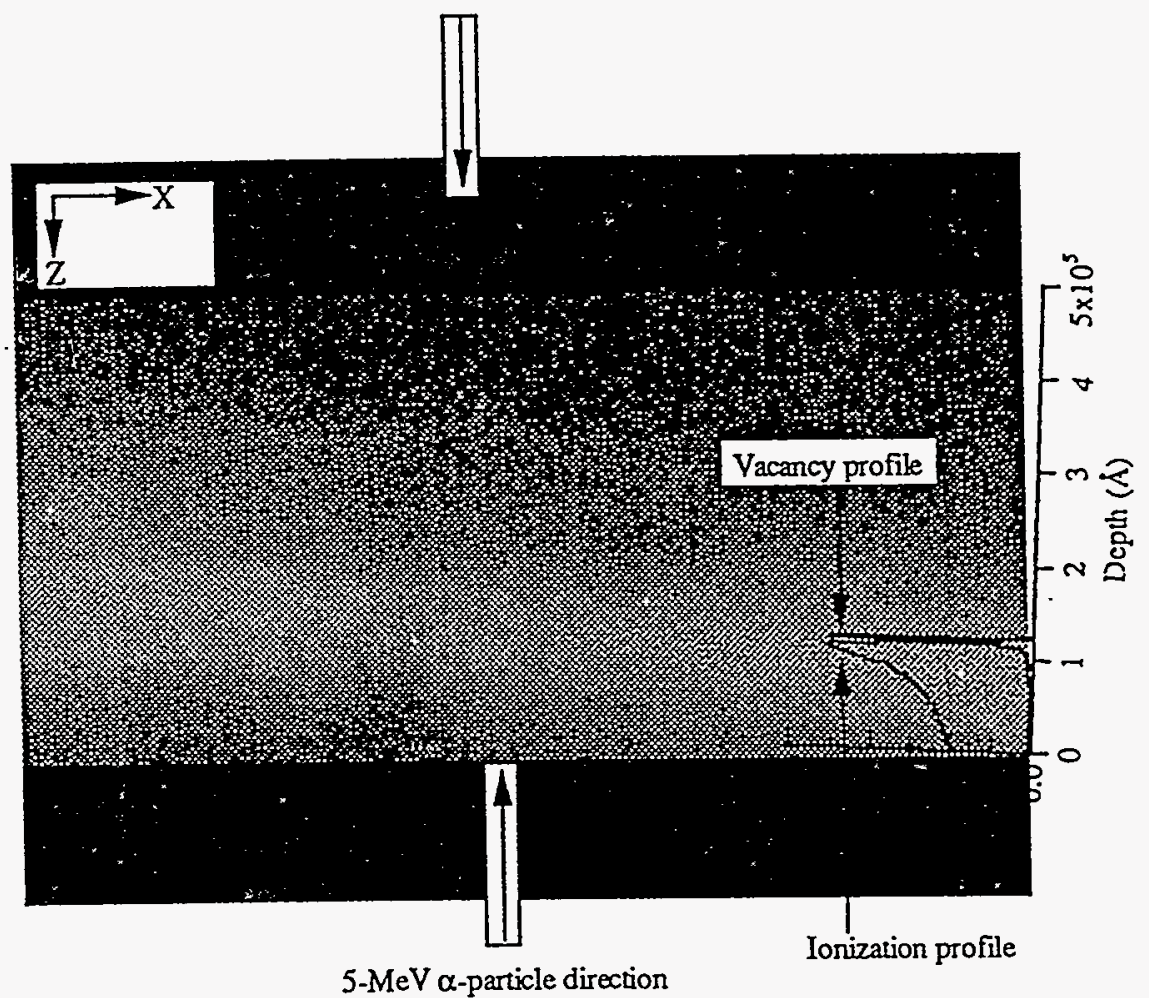

Figure D.4: Confocal micrograph of the damaged region from the single crystal, natural type Ma diamond. The H3 PL line was used to obtain the micrograph. a) shows the surface image of the damaged region. The transmission lines are also imaged due to the PL line being reflected from the metal/semiconductor interface. b) shows the depth profile of the damaged region. 
sample 1, the Raman spectrum $\mathbf{b}$ (unirradiated) is identical to that of the Raman spectrum a (irradiated) indicating no detectable lattice damage. The same is true for sample 2, with a fluence of $1.6 \times 10^{13} \mathrm{~cm}^{-2}$, except for a higher background, from the increase in the number of vacancies produced and no distinct PL peak is observed in the damaged region. For sample 3, we again see higher background in Raman spectrum c (irradiated) compared to Raman spectrum d (unirradiated) due to vacancy production. In all spectra, whether the sample is damaged or undamaged, the widths of the Raman peaks stay constant at $4.2 \mathrm{~cm}^{-1}$ whenever the peaks are resolvable.

Spatially-resolved Raman/PL spectra were used to obtain profiles of H3 PL peak from samples 3,4 and 5 with the fluence range of $1.6 \times 10^{14} \mathrm{~cm}^{-2}$ to $1.6 \times 10^{15} \mathrm{~cm}^{-2}$ (Fig. D.6). The width of the damaged region of these samples corresponds to the width of the collimator used in the irradiation. The Raman/PL spectrum of sample 5 is similar to the PL spectrum of a $2 \mathrm{MeV} \mathrm{He}^{+}$-damaged CVD film at the fluence of $2.1 \times 10^{15} \mathrm{~cm}^{-2}$ obtained by Perry et al. [16]. Since the Raman peak widths do not change as a function of fluence, it is possible to use the intensity of $\mathrm{H} 3 \mathrm{PL}$ line as an indicator to assess the extent of the damage caused by radiation. Indeed, the intensities of H3 PL peaks are found to vary linearly with fluence. Also, the emergence of H3 PL peaks in samples 3 and 4 and not in sample 2 seems to suggest that the threshold fluence for the production of the $\mathrm{H} 3$ center is approximately $1 \times 10^{14} \mathrm{~cm}^{-2}$. Similar results are seen with natural, single-crystal diamonds. This is consistent with the work by Kozlov et al. [17] which demonstrated that a natural diamond detector can withstand up to a few times $10^{14} \mathrm{~cm}^{-2}$ of neutron fluence before appreciable degradation of the detector performance.

Fig. D.7 shows the Raman/PL spectra from single-crystal natural diamond and CVD diamond film sample 4, each having been exposed to a fluence of $1.6 \times 10^{14} \mathrm{~cm}^{-2}$. The features of the spectra are very similar but the ratio of the Raman (R) line intensity to the H3 PL line intensity for CVD film divided by the same ratio for natural diamond is 
0.7 , suggesting that the polycrystalline CVD films may damage faster by a factor of approximately 1.4 than the single-crystal natural diamond.

Fig. D.8-i shows TPC signals obtained from damaged and undamaged natural diamond. The signal amplitude, which is proportional to the mobility-lifetime product of the free carriers, is seen to be reduced by a factor of three as a result of the radiation damage even when the laser probe depth is much less than the location of the Bragg peak. Similar effects are seen for irradiated CVD diamond (Fig. D.8-ii). The TPC signal from the most highly damaged specimen (sample 5) is smaller by a factor of two compared to that from an unirradiated specimen (sample 0). If we scale this to the fluence used for the damaged specimen in Fig. D.8-i, a signal reduction by a factor of eight is expected.

These measurements are consistent with results obtained from the Raman/PL measurements.

One feature interesting feature to note in Fig. D.8-ii is that the pulses for the samples 0 and 2 show some long-lived pulse tailing whereas this characteristic is missing from samples 4 and 5. The long tailing from CVD diamonds is attributed to the trapping/detrapping phenomenon occurring from shallow lying defect centers arising from crystal defects and graphite incorporation during the growth. The tailing is also present, to a small extent, in some samples of single-crystal, natural diamonds. By introducing additional deep trap/recombination centers (the damaged sites) through irradiation, the detrapping of the carriers is eliminated. This type of behavior is also seen by Wagner [18] when a natural diamond sample is neutron-damaged and excited with a pulse of protons.

\section{Conclusion}

Radiation damage induced by $\alpha$-particle bombardment has been studied in singlecrystal natural type Ila diamonds and polycrystalline CVD diamonds through Raman and 
PL spectroscopies and TPC. Lattice dàmage is apparent above fluences of $1 \times 10^{14} \mathrm{~cm}^{-2}$ from the emergence and dominance of H3 PL peak in Raman/PL spectra. From TPC measurements, we see a dramatic effect of the damage on electrical properties. It appears that polycrystalline CVD films for these measurements are more sensitive to radiation damage than natural, type IIa single-crystal diamond. Finally, it is possible to image the damaged regions using the H3 PL line and confocal fluorescence microscopy. The depth profiling done through it corresponds well with the calculated vacaney production profile. 

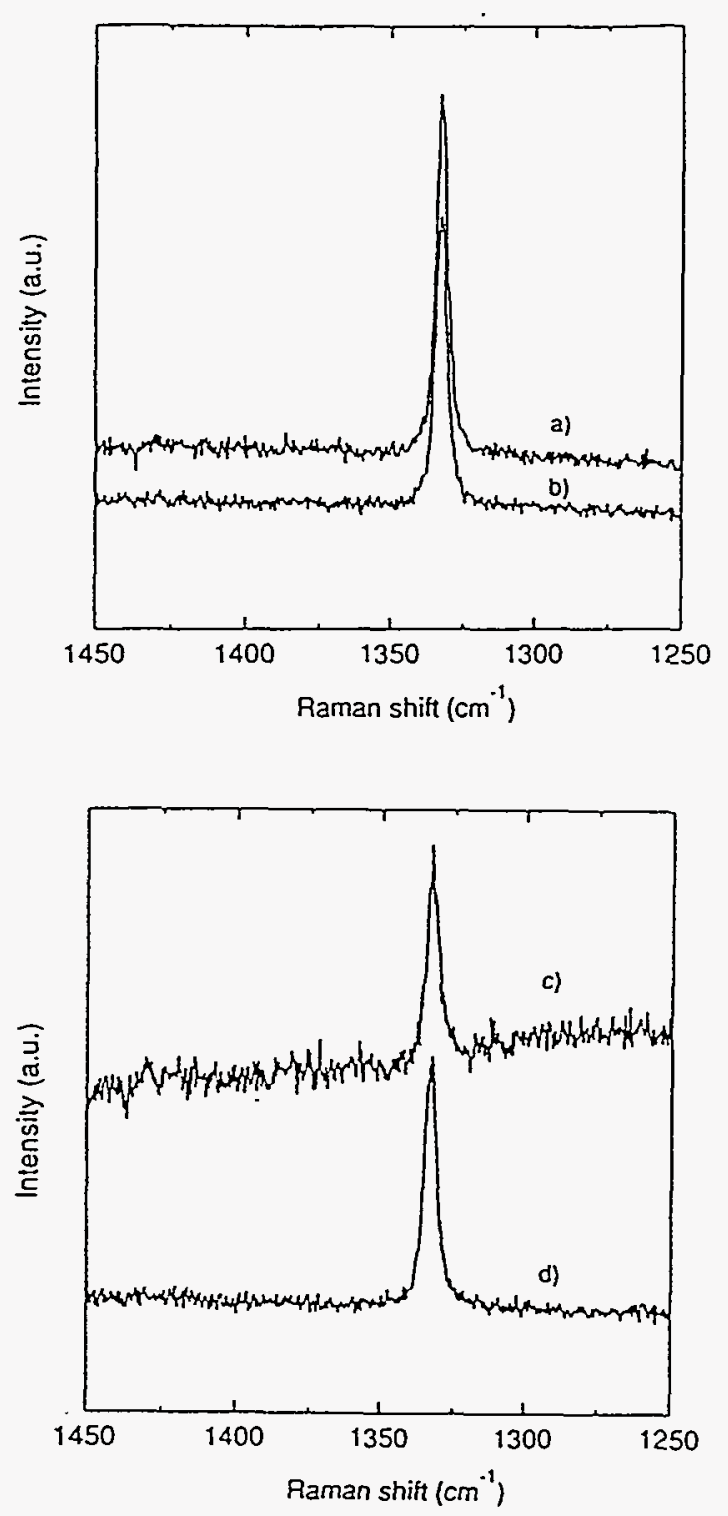

Figure D.5: Raman spectra of samples 1 and 3 with fluences of $1.6 \times 10^{12} \mathrm{~cm}^{-2}$ and $1.6 \times 1014 \mathrm{~cm}^{-2}$, respectively. Spectra a) and b) are the spectra taken from damaged and undamaged, respectively, regions of sample 1. Spectra c) and d) are the spectra taken from damaged and undamaged, respectively, regions of sample 3. The widths of the Raman peaks remained constant at $4.2 \mathrm{~cm}^{-1}$. a) and b) are identical indicating no detectable damage and c) shows higher background compared to d). 


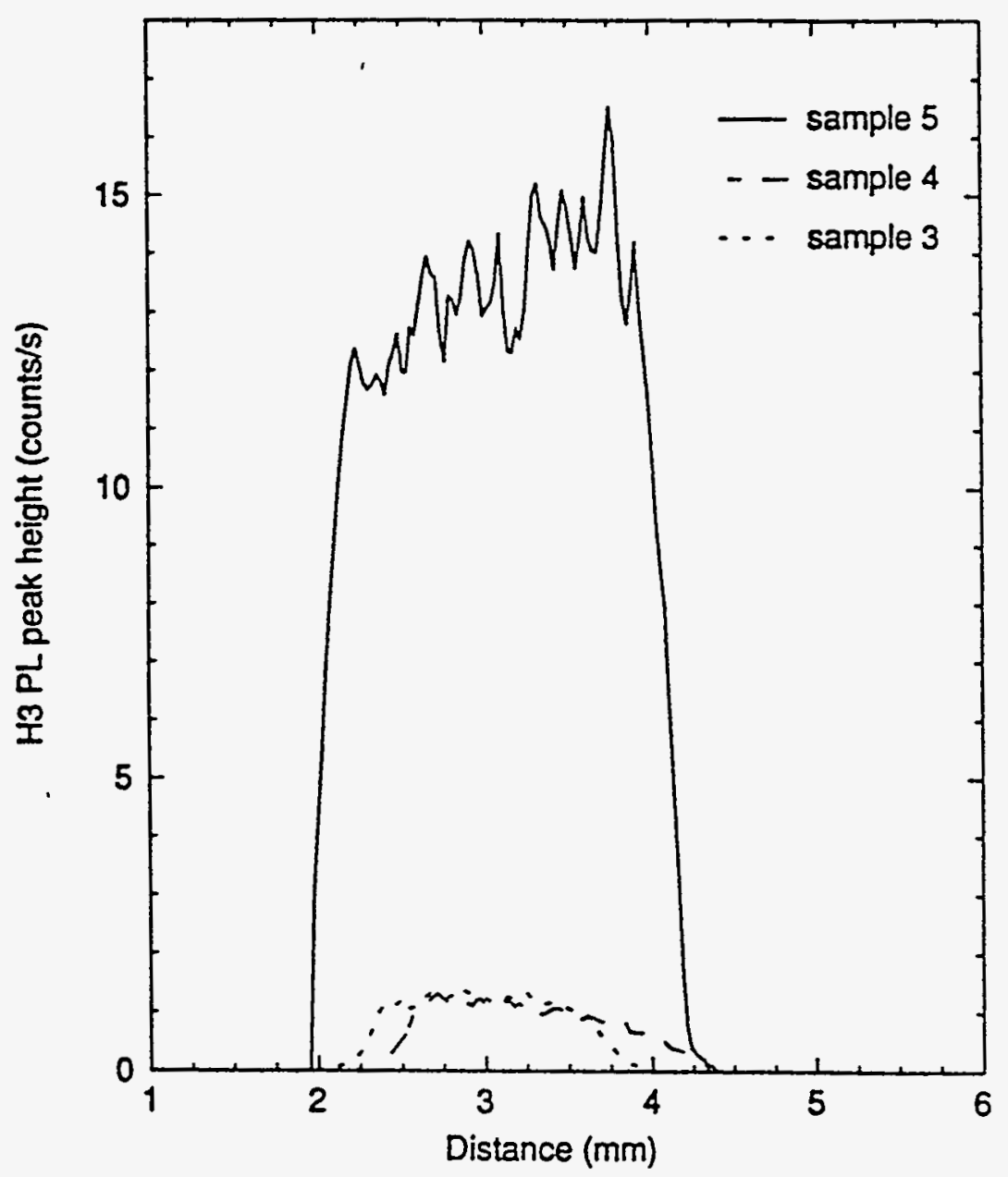

Figure D.6: Spatially-resolved H3 PL peaks across the damaged regions of sample 3,4 and 5 with fluences of $1.6 \times 10^{14} \mathrm{~cm}^{-2}, 1.6 \times 10^{14} \mathrm{~cm}^{-2}$ and $1.6 \times 1015 \mathrm{~cm}^{-2}$, respectively. The intensities of $\mathrm{H} 3 \mathrm{PL}$ peaks are linear as a function of ion fluence. $1 \mathrm{~mm}$ denotes the start and $6 \mathrm{~mm}$ denotes the end of the laser line illumination. 


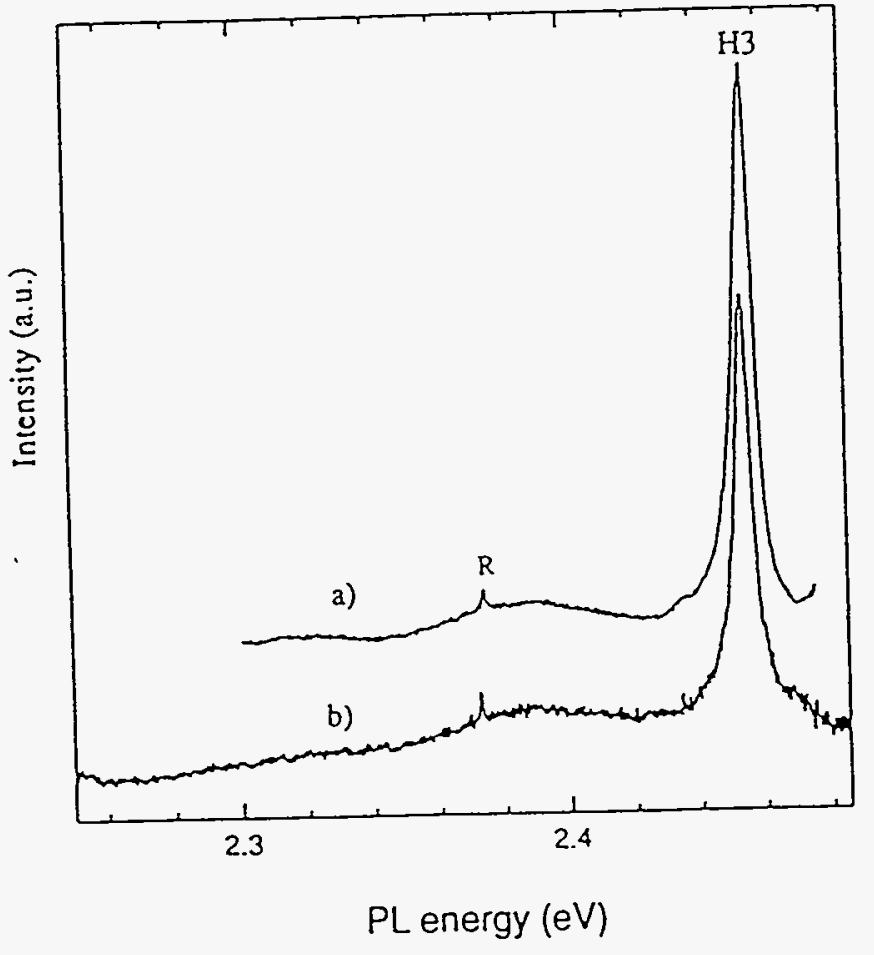

Figure D.7: Raman/PL spectra of polycrystalline CVD diamond, denoted with a), and single-crystal natural type IIa diamond, denoted with b). The ion fluence for both of the samples is $1.6 \times 10^{14} \mathrm{~cm}^{-2}$. 

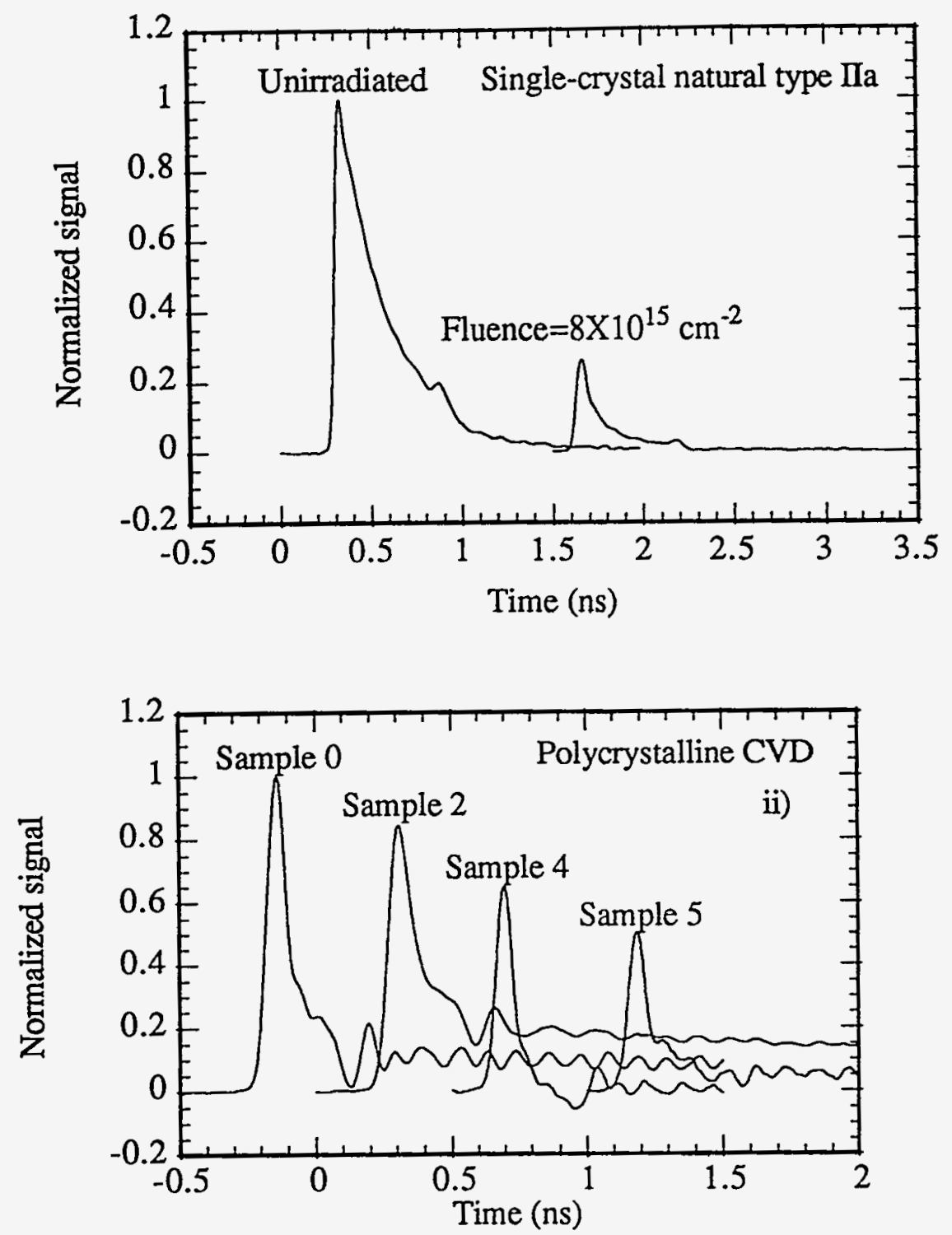

Figure D.8: Transient photoconductivity signals from damaged and undamaged samples. i) denotes the photoconductivity signals from a singlecrystal natural type Ma diamond and ii) denotes the photoconductivity signals from polycrystalline CVD diamond films. Sample 0 in ii) denotes the unirradiated CVD diamond. 
[1] V. A. J. van Lint, T. M. Flanagan, R. E. Leadon, J. A. Naber and V. C. Rogers, Mechanisms of Radiation Effects in Electronic Materials, Vol. 1, John Wiley \& Sons, New York, pp 270

[2] DIAMAS SSC Collaboration, Grant proposal SSC EOI-9, May 1990

[3] M. Franklin, A. Fry, S. Han, H. Kagan, S. Kanda, D. Kania, R. Kass, S. K. Kim, R. Malchow, F. Morrow, S. Olsen, W. F. Palmer, L. S. Pan, F. Sannes, S. Schnetzer, R. Stone, Y. Sugimoto, G. B. Thomson, C. White and S. Zhao, NIM A315, (1992) 39

[4] L. S. Pan, S. Han, D. R. Kania, M. A. Plano and M. I. Landstrass, to be published in Diamond and Related Materials

[5] J. Koike, D. M. Parkin and T. E. Mitchell, Appl. Phys. Lett., 60(12) (1992) 1450

[6] For a review on radiation damage in natural diamond see J. W. Corbett, Solid State Physics: Electron Radiation Damage in Semiconductors and Metals, Sup. 7, Academic Press, New York, 1966

[7] J. E. Field, ed., The Properties of Diamond, Academic Press, New York, (1979).

[8] L. S. Pan, D. R. Kania, S. Han, J. W. Ager II, M. Landstrass, O. L. Landen, and P. Pianetta, Science, 255, (1992) 830

[9] Crystallume Inc., 125 Constitution Drive, Menlo Park, California, 94025, U.S. A.

[10] M. Hoinkis, E. R. Weber, M. I. Landstrass, M. A. Plano, S. Han and D. R. Kania, Appl. Phys. Lett., 50(15), (1991) 1870

[11] J. W. Ager III, D. K. Veirs and G. M. Rosenblatt, Phys. Rev. B, 43 (1991) 6491

[12] D. K. Veirs, J. W. Ager III, E. T. Loucks and G. M. Rosenblatt, Appl. Opt. 29, No. 33, (1990) 4969

[13] Olympus LSM-GB200, Equipment manual

[14] M. Adel, R. Kalish and V. Richter, J. Mater. Res., 1(3) (1986) 503 - 234 - 
[15] D. R. Olander, Fundamental Aspects of Nuclear Reactor Fuel Elements, Energy Research and Development Administration, (1976) Ch. 7.3 - 7.5.

[16] T: A. Perry and C. P. Beetz, Jr., SPIE Proceedings: Raman Scattering, Luminescence and Spectroscopic Instrumentation in Technology, 1055 (1989) 152

[17] S. F. Kozlov, R. Stuck, M. Hage-Ali and P. Sieffert, IEEE Trans. Nucl. Sci. NS22 (1975) 160.

[18] R. S. Wagner, personal communication 


\section{Appendix E:}

Calculation of the ratio of the peak carrier density to the mean for carriers that are distributed in a Gaussian distribution radially within an ion track 
The ion track is assumed to be a cylinder with the mean volume, $\mathrm{V}$, in which all of the initial mean number of carriers produced, $N$, is contained. The carriers are assumed to be distributed uniformly along the ion trajectory and to be distributed in a Gaussian form in the radial direction. Then, the carrier density as a function of radial direction, r, (Fig. E.1) is given by

$$
n(r)=\frac{k}{\sqrt{2 \pi} \sigma} e^{-(r-F)^{2} / 2 / \sigma^{2}} ; r \geq 0
$$

where $\mathrm{k}=$ constant

$$
\begin{aligned}
& \sigma=\text { standard deviation } \\
& \bar{r}=\text { centroid of the Gaussian distribution function }
\end{aligned}
$$

By placing the origin at the center of the disk shown in Fig. E.1-A, the density distribution (Equ. E.1) has its peak at $r=0$ (Fig. E. 1-B). Therefore, the peak density can be expressed as

$$
n_{o}=\frac{k}{\sqrt{2 \pi} \sigma}
$$

Equ. E.2 is substituted into Equ. E.1 which results in

$$
n(r)=n_{0} e^{-\left(r^{2} / 2 / \sigma^{2}\right)} ; \mathrm{r} \geq 0
$$

The mean carrier density is defined to be the mean density over the volume of the ion track and is given by

$$
\langle n\rangle=\frac{\int n(r) d V}{\int d V}
$$

where $d V=R d A$

$$
\begin{aligned}
\mathrm{R} & =\text { mean ion range } \\
\mathrm{dA} & =\text { differential area normal to the ion trajectory } \\
& =2 \pi \mathrm{rdr}
\end{aligned}
$$


(A)

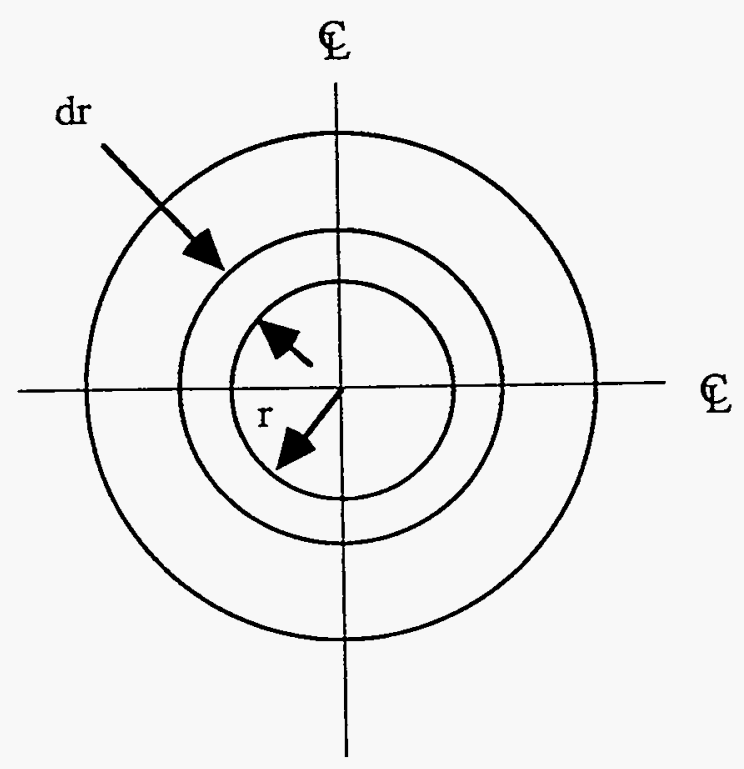

B)

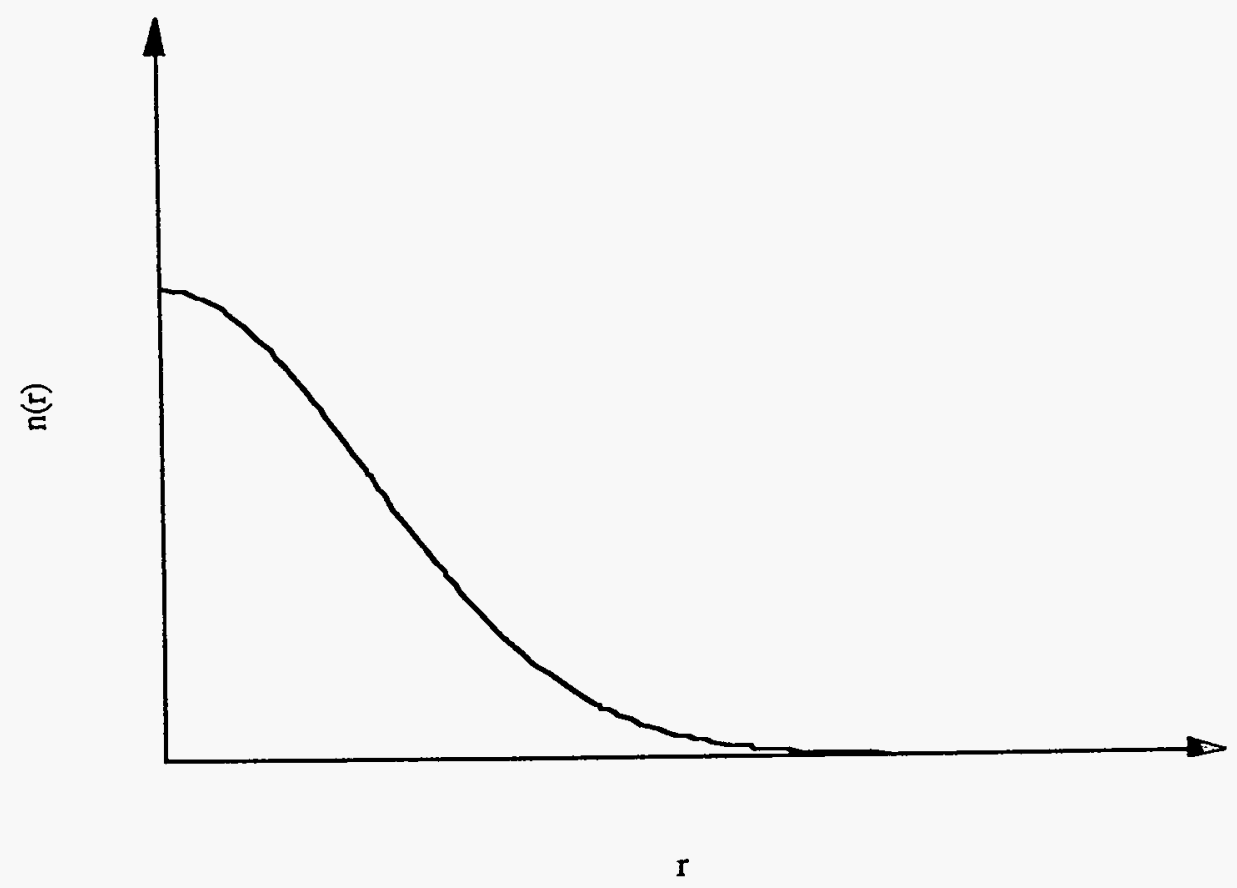

Figure E.1: Schematic of carrier denşity distribution in the radial direction; (A) area of the ion track normal to its trajectory; (B) assumed carrier distribution in the radial direction. 
Substituting Equ. E.3 into Equ. E.4 along with the integration limits of zero and infinity, the mean density is given by

$$
\langle n\rangle=\frac{n_{0}^{\infty} e^{-\left(r^{2} / 2 / \sigma^{2}\right)} r d r}{\int_{0}^{\infty} r d r}
$$

By assuming that the radius of the ion track is approximated by the FWHM $(=2.355 \sigma)$ of the carrier density distribution, the denominator in E.5 is approximately expressed as

$$
\int_{0}^{\infty} r d r \approx \frac{(2.355 \sigma)^{2}}{2}
$$

Substituting Equ. E.6 into Equ. E.5 and solving the definite integral in the numerator of E.5, the mean carrier density is given by

$$
\langle n\rangle=0.362 n_{o}
$$

Therefore, the ratio of the peak carrier density to the mean is just

$$
\frac{n_{o}}{\langle n\rangle}=2.760
$$

With Equ. E.8, the peak density of the carrier density distribution can be calculated simply by knowing the mean values of the ion range (R), ion track radius (FWHM) and energy needed to produce an electron-hole pair $(\varepsilon)$, and the total energy deposited $(E)$, i.e.,

$$
\langle n\rangle=\left(\frac{E}{\varepsilon}\right)\left(\frac{1}{\pi(F W H M)^{2} R}\right)
$$

